\title{
ON THE NON-EXISTENCE OF ELEMENTS OF KERVAIRE INVARIANT ONE
}

\author{
M. A. HILL, M. J. HOPKINS, AND D. C. RAVENEL \\ Dedicated to Mark Mahowald
}

\begin{abstract}
We show that the Kervaire invariant one elements $\theta_{j} \in \pi_{2^{j+1}-2} S^{0}$ exist only for $j \leq 6$. By Browder's Theorem, this means that smooth framed manifolds of Kervaire invariant one exist only in dimensions 2, 6, 14, 30, 62, and possibly 126. Except for dimension 126 this resolves a longstanding problem in algebraic topology.
\end{abstract}

\section{Contents}

1. Introduction

2. Equivariant stable homotopy theory

3. Mackey functors, homology and homotopy 35

4. The slice filtration 44

5. The complex cobordism spectrum 63

6. The Slice Theorem and the Reduction Theorem 76

7. The Reduction Theorem 80

8. The Gap Theorem 88

9. The Periodicity Theorem 89

10. The Homotopy Fixed Point Theorem 101

11. The Detection Theorem 103

Appendix A. The category of equivariant orthogonal spectra 118

Appendix B. Homotopy theory of equivariant orthogonal spectra 143

References 217

\section{INTRODUCTION}

The existence of smooth framed manifolds of Kervaire invariant one is one of the oldest unresolved issues in differential and algebraic topology. The question originated in the work of Pontryagin in the 1930's. It took a definitive form in the paper [41] of Kervaire in which he constructed a combinatorial 10-manifold with no smooth structure, and in the work of Kervaire-Milnor [42] on $h$-cobordism classes

M. A. Hill was partially supported by NSF grants DMS-0905160, DMS-1307896 and the Sloan foundation.

M. J. Hopkins was partially supported the NSF grant DMS-0906194.

D. C. Ravenel was partially supported by the NSF grants DMS-1307896 and DMS-0901560.

All three authors received support from the DARPA grants HR0011-10-1-0054-DOD35CAP and FA9550-07-1-0555. 
of manifolds homeomorphic to a sphere. The question was connected to homotopy theory by Browder in his fundamental paper [13] where he showed that smooth framed manifolds of Kervaire invariant one exist only in dimensions of the form $\left(2^{j+1}-2\right)$, and that a manifold exists in that dimension if and only if the class

$$
h_{j}^{2} \in \operatorname{Ext}_{\mathcal{A}}^{2,2^{j+1}}(\mathbb{Z} / 2, \mathbb{Z} / 2)
$$

in the $E_{2}$-term of the classical Adams spectral represents an element

$$
\theta_{j} \in \pi_{2^{j+1}-2} S^{0}
$$

in the stable homotopy groups of spheres. The classes $h_{j}^{2}$ for $j \leq 3$ represent the squares of the Hopf maps. The element $\theta_{4} \in \pi_{30} S^{0}$ had been observed in existing computations [56, 52,60], and was constructed explicitly as a framed manifold by Jones [39]. The element $\theta_{5} \in \pi_{60} S^{0}$ was constructed by Barratt-Mahowald, and Barratt-Jones-Mahowald (see [9] and the discussion therein).

The purpose of this paper is to prove the following theorem

Theorem 1.1. For $j \geq 7$ the class $h_{j}^{2} \in \operatorname{Ext}_{\mathcal{A}}^{2,2^{j+1}}(\mathbb{Z} / 2, \mathbb{Z} / 2)$ does not represent an element of the stable homotopy groups of spheres. In other words, the Kervaire invariant elements $\theta_{j}$ do not exist for $j \geq 7$.

Smooth framed manifolds of Kervaire invariant one therefore exist only in dimensions $2,6,14,30,62$, and possibly 126 . At the time of writing, our methods still leave open the existence of $\theta_{6}$.

Many open issues in algebraic and differential topology depend on knowing whether or not the Kervaire invariant one elements $\theta_{j}$ exist for $j \geq 6$. The following results represent some of the issues now settled by Theorem 1.1. In the statements, the phrase "exceptional dimensions" refers to the dimensions $2,6,14$, 30,62 , and 126. In all cases the situation in the dimension 126 is unresolved. By Browder's work [13] the results listed below were known when the dimension in question was not 2 less than a power of 2. Modulo Browder's result [13] the reduction of the statements to Theorem 1.1 can be found in the references cited.

Theorem 1.2 ([42, 46]). Except in the six exceptional dimensions, every stably framed smooth manifold is framed cobordant to a homotopy sphere.

In the first five of the exceptional dimensions it is known that not every stably framed manifold is framed cobordant to a homotopy sphere. The situation is unresolved in dimension 126.

Theorem 1.3 ([42]). Let $M^{m}$ be the manifold with boundary constructed by plumbing together two copies of the unit tangent bundle to $S^{2 k+1}($ so $m=4 k+2)$, and set $\Sigma^{m-1}=\partial M^{m}$. Unless $m$ is one of the six exceptional dimensions, the space $M^{m} / \Sigma^{m-1}$ is a triangulable manifold which does not admit any smooth structure, and the manifold $\Sigma^{m-1}$ (the Kervaire sphere) is homoemorphic but not diffeomorphic to $S^{m-1}$.

In the first five of the exceptional cases, the Kervaire sphere is known to be diffeomorphic to the ordinary sphere, and the Kervaire manifold can be smoothed.

Theorem 1.4 ([42, 46]). Let $\Theta_{n}$ be the Kervaire-Milnor group of h-cobordism classes of homotopy $n$-spheres. Unless $(4 k+2)$ is one of the six exceptional dimensions,

$$
\Theta_{4 k+2} \approx \pi_{4 k+2} S^{0}
$$


and

$$
\left|\Theta_{4 k+1}\right|=a_{k}\left|\pi_{4 k+1} S^{0}\right|,
$$

where $a_{k}$ is 1 if $k$ is even, and 2 if $k$ is odd.

Theorem 1.5 ([8]). Unless $n$ is 1 , or one of the six exceptional dimensions, the Whitehead square $\left[\iota_{n+1}, \iota_{n+1}\right] \in \pi_{2 n+1} S^{n+1}$ is not divisible by 2 .

1.1. Outline of the argument. Our proof builds on the strategy used by the third author in [70] and on the homotopy theoretic refinement developed by the second author and Haynes Miller (see [73]).

We construct a multiplicative cohomology theory $\Omega$ and establish the following results:

Theorem 1.6 (The Detection Theorem). If $\theta_{j} \in \pi_{2^{j+1}-2} S^{0}$ is an element of Kervaire invariant 1 , and $j>2$, then the "Hurewicz" image of $\theta_{j}$ in $\Omega^{2-2^{j+1}}(p t)$ is non-zero.

Theorem 1.7 (The Periodicity Theorem). The cohomology theory $\Omega$ is 256-fold periodic: For all $X$,

$$
\Omega^{*}(X) \approx \Omega^{*+256}(X) .
$$

Theorem 1.8 (The Gap Theorem). The groups $\Omega^{i}(p t)$ are zero for $0<i<4$.

These three results easily imply Theorem 1.1. The Periodicity Theorem and the Gap Theorem imply that the groups $\Omega^{i}(\mathrm{pt})$ are zero for $i \equiv 2 \bmod 256$. By the Detection Theorem, if $\theta_{j}$ exists it has a non-zero Hurewicz image in $\Omega^{2-2^{j+1}}(\mathrm{pt})$. But this latter group is zero if $j \geq 7$.

1.2. The cohomology theory $\Omega$. Write $C_{n}$ for the cyclic group of order $n$. Our cohomology theory $\Omega$ is part of a pair $\left(\Omega, \Omega_{\mathbb{O}}\right)$ analogous to the orthogonal and unitary $K$-theory spectra $K O$ and $K U$. The role of complex conjugation on $K U$ is played by an action of $C_{8}$ on $\Omega_{\mathbb{O}}$, and $\Omega$ arises as its fixed points. It is better to think of $\Omega_{\mathbb{O}}$ as generalizing Atiyah's $C_{2}$-equivariant $K_{\mathbb{R}}$-theory [7], and in fact $\Omega_{\mathbb{O}}$ is constructed from the corresponding real bordism spectrum, as we now describe.

Let $M U_{\mathbb{R}}$ be the $C_{2}$-equivariant real bordism spectrum of Landweber [43] and Fujii [24]. Roughly speaking one can think of $M U_{\mathbb{R}}$ as describing the cobordism theory of real manifolds, which are stably almost complex manifolds equipped with a conjugate linear action of $C_{2}$, such as the space of complex points of a smooth variety defined over $\mathbb{R}$. A real manifold of real dimension $2 n$ determines a homotopy class of maps

$$
S^{n \rho_{2}} \rightarrow M U_{\mathbb{R}}
$$

where $n \rho_{2}$ is the direct sum of $n$ copies of the real regular representation of $C_{2}$, and $S^{n \rho_{2}}$ is its one point compactification.

Write

$$
M U^{\left(\left(C_{8}\right)\right)}=M U_{\mathbb{R}} \wedge M U_{\mathbb{R}} \wedge M U_{\mathbb{R}} \wedge M U_{\mathbb{R}}
$$

for the $C_{8}$-equivariant spectrum gotten by smashing 4 copies of $M U_{\mathbb{R}}$ together and letting $C_{8}$ act by

$$
(a, b, c, d) \mapsto(\bar{d}, a, b, c) .
$$

Very roughly speaking, $M U^{\left(\left(C_{8}\right)\right)}$ can be thought of as the cobordism theory of stably almost manifolds equipped with a $C_{8}$-action, with the property that the 
restriction of the action to $C_{2} \subset C_{8}$ determines a real structure. If $M$ is a real manifold then $M \times M \times M \times M$ with the $C_{8}$-action

$$
(a, b, c, d) \mapsto(\bar{d}, a, b, c)
$$

is an example. A suitable $C_{8}$-manifold $M$ of real dimension $8 n$ determines a homotopy class of maps

$$
S^{n \rho_{8}} \rightarrow M U^{\left(\left(C_{8}\right)\right)},
$$

where $n \rho_{8}$ is the direct sum of $n$ copies of the real regular representation of $C_{8}$, and $S^{n \rho_{8}}$ is its one point compactification.

To define $\Omega$ we invert an equivariant analogue

$$
D: S^{\ell \rho_{8}} \rightarrow M U^{\left(\left(C_{8}\right)\right)}
$$

of the Bott periodicity class and form the $C_{8}$-equivariant spectrum $\Omega_{\mathbb{O}}=D^{-1} M U^{\left(\left(C_{8}\right)\right)}$ (in fact $\ell$ works out to be 19). The cohomology theory $\Omega$ is defined to be the homotopy fixed point spectrum of the $C_{8}$-action on $\Omega_{\mathbb{O}}$.

There is some flexibility in the choice of $D$, but it needs to be chosen in order that the Periodicity Theorem holds, and in order that the map from the fixed point spectrum of $\Omega_{\mathbb{O}}$ to the homotopy fixed point spectrum be a weak equivalence. It also needs to be chosen in such a way that the Detection Theorem is preserved (see Remark 11.14). That such an $D$ can be chosen with these properties is a relatively easy fact, albeit mildly technical. It is specified in Corollary 9.21. It can be described in the form $M \times M \times M \times M$ for a suitable real manifold $M$, though we do not do so.

1.3. The Detection Theorem. Since the non-equivariant spectrum $\Omega_{\mathbb{O}}$ underlying $\Omega_{\mathbb{O}}$ is complex orientable, the inclusion of the unit $S^{0} \rightarrow \Omega$ induces a map

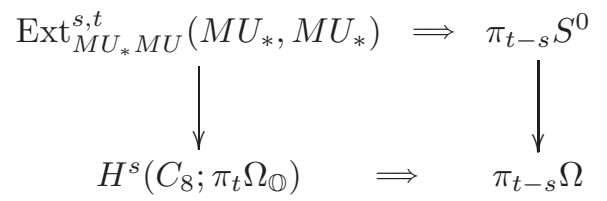

from the Adams-Novikov spectral sequence to the $C_{8}$ homotopy fixed point spectral sequence for $\pi_{*} \Omega$. In $\S 11.3 .3$ we give an ad hoc construction of this spectral sequence, conveniently adapted to describing the map of $E_{2}$-terms. It gives the horizontal arrow in the diagram of spectral sequences below.

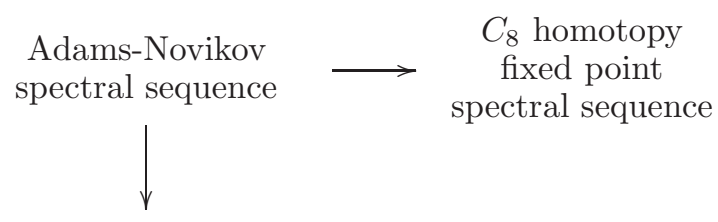

Classical Adams

spectral sequence

The Detection Theorem is proved by investigating this diagram, and follows from a purely algebraic result.

Theorem 1.9 (Algebraic Detection Theorem). If

$$
x \in \operatorname{Ext}_{M U_{*}(M U)}^{2,2^{j+1}}\left(M U_{*}, M U_{*}\right)
$$


is any element mapping to $h_{j}^{2}$ in the $E_{2}$-term of the classical Adams spectral sequence, and $j>2$, then the image of $x$ in $H^{2}\left(C_{8} ; \pi_{2^{j+1}} \Omega_{\mathbb{O}}\right)$ is nonzero.

The restriction $j>2$ is not actually necessary, but the other values of $j$ require separate arguments. Since we do not need them, we have chosen to leave them to the interested reader.

To deduce the Detection Theorem from the Algebraic Detection Theorem suppose that $\theta_{j}: S^{2^{j+1}-2} \rightarrow S^{0}$ is a map represented by $h_{j}^{2}$ in the classical Adams spectral sequence. Then $\theta_{j}$ has Adams filtration 0, 1 or 2 in the Adams-Novikov spectral sequence, since the Adams filtration can only increase under a map. Since both

$$
\operatorname{Ext}_{M U_{*} M U}^{0,2^{j+1}-2}\left(M U_{*}, M U_{*}\right) \quad \text { and } \quad \operatorname{Ext}_{M U_{*} M U}^{1,2^{j+1}-1}\left(M U_{*}, M U_{*}\right)
$$

are zero, the class $\theta_{j}$ must be represented in Adams filtration 2 by some element $x$ which is a permanent cycle. By the Algebraic Detection Theorem, the element $x$ has a non-trivial image $b_{j} \in H^{2}\left(C_{8} ; \pi_{2^{j+1}} \Omega_{\mathbb{O}}\right)$, representing the image of $\theta_{j}$ in $\pi_{2^{j+1}-2} \Omega$. If this image is zero then the class $b_{j}$ must be in the image of the differential

$$
d_{2}: H^{0}\left(C_{8} ; \pi_{2^{j+1}-1} \Omega_{\mathbb{O}}\right) \rightarrow H^{2}\left(C_{8} ; \pi_{2^{j+1}} \Omega_{\mathbb{O}}\right) .
$$

But $\pi_{\text {odd }} \Omega_{\mathbb{O}}=0$, so this cannot happen.

The proof of the Algebraic Detection Theorem is given in $\S 11$. The method of proof is similar to that used in [70], where an analogous result is established at primes greater than 3 .

1.4. The slice filtration and the Gap Theorem. While the Detection Theorem and the Periodicity Theorem involve the homotopy fixed point spectral sequence for $\Omega$, the Gap Theorem results from studying $\Omega_{\mathbb{O}}$ as an honest equivariant spectrum. What permits the mixing of the two approaches is the following result, which is part of Theorem 10.8.

Theorem 1.10 (Homotopy Fixed Point Theorem). The map from the fixed point spectrum of $\Omega_{\mathbb{O}}$ to the homotopy fixed point spectrum of $\Omega_{\mathbb{O}}$ is a weak equivalence.

In particular, for all $n$, the map

$$
\pi_{n}^{C_{8}} \Omega_{\mathbb{O}} \rightarrow \pi_{n} \Omega_{\mathbb{O}}^{h C_{8}}=\pi_{n} \Omega
$$

is an isomorphism, in which the symbol $\pi_{n}^{C_{8}} \Omega_{\mathbb{O}}$ denotes the group of equivariant homotopy classes of maps from $S^{n}$ (with the trivial action) to $\Omega_{\mathbb{O}}$.

We study the equivariant homotopy type of $\Omega_{\mathbb{O}}$ using an analogue of the Postnikov tower. We call this tower the slice tower. Versions of it have appeared in work of Dan Dugger [20], Hopkins-Morel (unpublished), Voevodsky[83, 81, 82], and Hu-Kriz [35].

The slice tower is defined for any finite group $G$. For a subgroup $K \subset G$, let $\rho_{K}$ denote its regular representation and write

$$
\widehat{S}(m, K)=G_{+} \underset{K}{\wedge} S^{m \rho_{K}} \quad m \in \mathbb{Z} .
$$

Definition 1.11. The set of slice cells (for $G$ ) is

$$
\left\{\widehat{S}(m, K), \Sigma^{-1} \widehat{S}(m, K) \mid m \in \mathbb{Z}, K \subset G\right\} .
$$

Definition 1.12. A slice cell $\widehat{S}$ is free if it is of the form $G_{+} \wedge S^{m}$ for some $m$. An isotropic slice cell is one which is not free. 
We define the dimension of a slice cell $\widehat{S}$ by

$$
\begin{aligned}
\operatorname{dim} \widehat{S}(m, K) & =m|K| \\
\operatorname{dim} \Sigma^{-1} \widehat{S}(m, K) & =m|K|-1 .
\end{aligned}
$$

Finally the slice section $P^{n} X$ is constructed by attaching cones on slice cells $\widehat{S}$ with $\operatorname{dim} \widehat{S}>n$ to kill all maps $\widehat{S} \rightarrow X$ with $\operatorname{dim} \widehat{S}>n$. There is a natural map

$$
P^{n} X \rightarrow P^{n-1} X
$$

The $n$-slice of $X$ is defined to be its homotopy fiber $P_{n}^{n} X$.

In this way a tower $\left\{P^{n} X\right\}, n \in \mathbb{Z}$ is associated to each equivariant spectrum $X$. The homotopy colimit holim $P^{n} X$ is contractible, and holim ${ }_{n} P^{n} X$ is just $X$. The slice spectral sequence for $\vec{X}^{n}$ is the spectral sequence of the slice tower, relating $\pi_{*} P_{n}^{n} X$ to $\pi_{*} X$.

The key technical result of the whole paper is the following.

Theorem 1.13 (The Slice Theorem). The $C_{8}$-spectrum $P_{n}^{n} M U^{\left(\left(C_{8}\right)\right)}$ is contractible if $n$ is odd. If $n$ is even then $P_{n}^{n} M U^{\left(\left(C_{8}\right)\right)}$ is weakly equivalent to $H \underline{\mathbb{Z}} \wedge W$, where $H \underline{\mathbb{Z}}$ is the Eilenberg-Mac Lane spectrum associated to the constant Mackey functor $\underline{\mathbb{Z}}$, and $W$ is a wedge of isotropic slice cells of dimension $n$.

The Slice Theorem actually holds more generally for the spectra $M U^{\left(\left(C_{2^{k}}\right)\right)}$ formed like $M U^{\left(\left(C_{8}\right)\right)}$, using the smash product of $2^{k-1}$ copies of $M U_{\mathbb{R}}$. The more general statement is Theorem 6.1

The Gap Theorem depends on the following result.

Lemma 1.14 (The Cell Lemma). Let $G=C_{2^{n}}$ for some $n \neq 0$. If $\widehat{S}$ is an isotropic slice cell of even dimension, then the groups $\pi_{k}^{G} H \underline{\mathbb{Z}} \wedge \widehat{S}$ are zero for $-4<k<0$.

This is an easy explicit computation, and reduces to the fact that the orbit space $S^{m \rho_{G}} / G$ is simply connected, being the suspension of a connected space.

Since the restriction of $\rho_{G}$ to a subgroup $K \subset G$ is isomorphic to $(|G / K|) \rho_{K}$ there is an equivalence

$$
S^{m \rho_{G}} \wedge\left(G_{+} \bigwedge_{K} S^{n \rho_{K}}\right) \approx G_{+} \bigwedge_{K}^{\wedge} S^{(n+m|G / K|) \rho_{K}} .
$$

It follows that if $\widehat{S}$ is a slice cell of dimension $d$, then for any $m, S^{m \rho_{G}} \wedge \widehat{S}$ is a slice cell of dimension $d+m|G|$. Moreover, if $\widehat{S}$ is isotropic, then so is $S^{m \rho_{G}} \wedge \widehat{S}$. The Cell Lemma and the Slice Theorem then imply that for any $m$, the group

$$
\pi_{i}^{C_{8}} S^{m \rho_{C_{8}}} \wedge M U^{\left(\left(C_{8}\right)\right)}
$$

is zero for $-4<i<0$. Since

this implies that

$$
\pi_{i}^{C_{8}} \Omega_{\mathbb{O}}=\lim _{i} \pi^{-m \rho_{\rho_{8}}} M U^{\left(\left(C_{8}\right)\right)}
$$

$$
\pi_{i}^{C_{8}} \Omega_{\mathbb{O}}=\pi_{i} \Omega=0
$$

for $-4<i<0$, which is the Gap Theorem.

The Periodicity Theorem is proved with a small amount of computation in the $R O\left(C_{8}\right)$-graded slice spectral sequence for $\Omega_{\mathbb{O}}$. It makes use of the fact that $\Omega_{\mathbb{O}}$ is an equivariant commutative ring spectrum. Using the nilpotence machinery of $[17,32]$ instead of explicit computation, it can be shown that the groups $\pi_{*} \Omega$ are periodic with some period which a power of 2 . This would be enough to show that only 
finitely many of the $\theta_{j}$ can exist. Some computation is necessary to get the actual period stated in the Periodicity Theorem.

All of the results are fairly easy consequences of the Slice Theorem, which in turn reduces to a single computational fact: that the quotient of $M U^{\left(\left(C_{8}\right)\right)}$ by the analogue of the "Lazard ring" is the Eilenberg-Mac Lane spectrum $H \underline{\mathbb{Z}}$ associated to the constant Mackey functor $\underline{\mathbb{Z}}$. We call this the Reduction Theorem and its generalization to $C_{2^{n}}$ appears as Theorem 6.5. It is proved for $G=C_{2}$ in $\mathrm{Hu}$ Kriz [35], and the analogue in motivic homotopy theory is the main result of the (unpublished) work of the second author and Morel mentioned earlier, where it is used to identify the Voevodsky slices of $M G L$. It would be very interesting to find a proof of Theorem 6.5 along the lines of Quillen's argument in [69].

During the long period between revisions of this paper, Haynes Miller's Bourbaki talk on this material has appeared [64]. We refer the reader there for a incisive overview.

1.5. Summary of the contents. We now turn to a more detailed summary of the contents of this paper. In $\S 2$ we recall the basics of equivariant stable homotopy theory, establish many conventions and explain some simple computations. One of our main new constructions, introduced in $\$ 2.2 .3$ is the multiplicative norm functor. We merely state our main results about the norm, deferring the details of the proofs to the appendices. Another useful technique, the method of twisted monoid rings, is described in \$2.4. It is used in constructing convenient filtrations of rings, and in forming the quotient of an equivariant commutative ring spectrum by a regular sequence, in the situation in which the group is acting non-trivially on the sequence.

Section 4 introduces the slice filtration, and establishes many of its basic properties, including the strong convergence of the slice spectral sequence (Theorem 4.42), and an important result on the distribution of groups in the $E_{2}$-term (Corollary 4.43). The notions of pure spectra, isotropic spectra, and spectra with cellular slices are introduced in $\$ 4.6 .2$. In these terms, the Slice Theorem states that $M U^{\left(\left(C_{2} n\right)\right)}$ is both pure and isotropic. Most of the material of these first sections makes no restriction on the group $G$.

From $\S 5$ forward we restrict attention to the case in which $G$ is cyclic of order a power of 2 , and we localize all spectra at the prime 2 . The spectra $M U^{((G))}$ are introduced and some of the basic properties are established. The groundwork is laid for the proof of the Slice Theorem. The Reduction Theorem (Theorem 6.5) is stated in $\S 6$. The Reduction Theorem is the backbone of the Slice Theorem, and is the only part that is not "formal" in the sense that it depends on the outcome of certain computations.

The Slice Theorem is also proved in $\S 6$, assuming that the Reduction Theorem holds. The proof of the Reduction Theorem is in $\S 7$. The Gap Theorem in proved in $\S 8$, the Periodicity theorem in $\S 9$. The Homotopy Fixed Point Theorem is proved in $\S 10$, and the Detection Theorem in $\S 11$.

The paper concludes with two appendices devoted to foundations of equivariant stable homotopy theory. Two factors contribute to the length of this material. One is simply the wish to make this paper as self-contained as possible and to collect material central to our investigation in one place. The other reason is that our methods rely on multiplicative aspects of equivariant stable homotopy theory that do not appear in the existing literature. Establishing the basic properties of these structures involve details of the foundations and cannot be done at the level of user 
interface. Because of this, a relatively complete account of equivariant orthogonal spectra is required.

1.6. Acknowledgments. First and foremost the authors would like to thank Ben Mann and the support of DARPA through the grant number FA9550-07-1-0555. It was the urging of Ben and the opportunity created by this funding that brought the authors together in collaboration in the first place. Though the results described in this paper were an unexpected outcome of our program, it's safe to say they would not have come into being without Ben's prodding. As it became clear that the techniques of equivariant homotopy theory were relevant to our project we drew heavily on the paper [35] of Po Hu and Igor Kriz. We'd like to acknowledge a debt of influence to that paper, and to thank the authors for writing it. We were also helped by the thesis of Dan Dugger (which appears as [20]). The second author would like to thank Dan Dugger, Marc Levine, Jacob Lurie, and Fabien Morel for several useful conversations. Early drafts of this manuscript were read by Mark Hovey, Tyler Lawson, and Peter Landweber, and the authors would like to express their gratitude for their many detailed comments. We also owe thanks to Haynes Miller for a very thoughtful and careful reading of our earlier drafts, and for his helpful suggestions for terminology. Thanks are due to Stefan Schwede for sharing with us his construction of $M U_{\mathbb{R}}$, to Mike Mandell for diligently manning the hotline for questions about the foundations of equivariant orthogonal spectra, to Andrew Blumberg for his many valuable comments on the second revision, and to Anna Marie Bohmann and Emily Riehl for valuable comments on our description of "working fiberwise."

Finally, and most importantly, the authors would like to thank Mark Mahowald for a lifetime of mathematical ideas and inspiration, and for many helpful discussions in the early stages of this project.

\section{Equivariant stable homotopy theory}

We will work in the category of equivariant orthogonal spectra $[54,53]$. In this section we survey some of the main properties of the theory and establish some notation. The definitions, proofs, constructions, and other details are explained in Appendices A and B. The reader is also referred to the books of tom Dieck [80, 79], and the survey of Greenlees and May [26] for an overview of equivariant stable homotopy theory, and for further references.

We set up the basics of equivariant stable homotopy theory in the framework of homotopical category in the sense of [21]. A homotopical category is a pair $(\mathcal{C}, \mathcal{W})$ consisting of a category $\mathcal{C}$ and a collection $\mathcal{W}$ of morphisms in $\mathcal{C}$ called weak equivalences containing all identity maps, and satisfying the "two out of six property" that in the situation

$$
\bullet \stackrel{u}{\rightarrow} \bullet \stackrel{v}{\rightarrow} \bullet \stackrel{w}{\rightarrow} \bullet
$$

if $v u$ and $w v$ are in $\mathcal{W}$ then so are $u, v, w$, and $v w u$. Any class $\mathcal{W}$ defined as the collection of morphisms $u$ taken to isomorphisms by some fixed functor automatically satisfies this property. This holds in particular when $\mathcal{W}$ consists of the weak equivalences in a model category structure. In this situation we will say that the model structure refines the homotopical category structure, and that the homotopical category is completed to a model category structure. 
Associated to a homotopical category $(\mathcal{C}, \mathcal{W})$ is the homotopy category ho $\mathcal{C}$ and the functor $\mathcal{C} \rightarrow$ ho $\mathcal{C}$, characterized uniquely up to unique isomorphism by the following universal property: for every category $\mathcal{D}$, and every functor $F: \mathcal{C} \rightarrow \mathcal{D}$ taking the stable weak equivalences as isomorphisms, there is a unique functor ho $\mathcal{C} \rightarrow D$ making the diagram

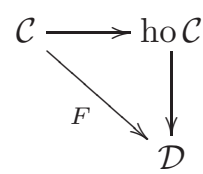

commute. See $\S B .1$ for more on the theory of homotopical categories, for a description of the issues that arise when doing homotopy theory in a homotopical category, the techniques for dealing with them, and for an explanation of the notion of left $(\mathbf{L})$ and right $(\mathbf{R})$ derived functors appearing in the discussion below.

2.1. $G$-spaces. We begin with unstable equivariant homotopy theory. Let $G$ be a finite group, and $\mathcal{T}^{G}$ the topological category of pointed compactly generated, weak Hausdorff left $G$-spaces and spaces of equivariant maps. The category $\mathcal{T}^{G}$ is a closed symmetric monoidal category under the smash product operation. The tensor unit is the 0 -sphere $S^{0}$ equipped with the trivial $G$-action.

We call a category enriched over $\mathcal{T}^{G}$ a $G$-equivariant topological category. Since it is closed monoidal, $\mathcal{T}^{G}$ may be regarded as enriched over itself. We denote the enriched category by $\mathcal{I}_{G}$. Thus $\mathcal{I}_{G}$ is the $G$-equivariant topological category of $G$-spaces and $G$-spaces of continuous, not necessarily equivariant maps, on which $G$ acts by conjugation. There is an isomorphism

$$
\mathcal{T}^{G}(X, Y)=\underline{\mathcal{T}}_{G}(X, Y)^{G} .
$$

See $\S A .1 .3$ and $\S A .2 .1$ for further background and discussion.

The homotopy set (group, for $n>0$ ) $\pi_{n}^{H}(X)$ of a pointed $G$-space is defined for $H \subset G$ and $n \geq 0$ to be the set of $H$-equivariant homotopy classes of pointed maps

$$
S^{n} \rightarrow X .
$$

This is the same as the ordinary homotopy set (group) $\pi_{n}\left(X^{H}\right)$ of the space of $H$ fixed-points in $X$.

A map $f: X \rightarrow Y$ in $\mathcal{T}^{G}$ is a weak equivalence if for all $H \subset G$ the map $X^{H} \rightarrow Y^{H}$ of $H$-fixed point spaces is an ordinary weak equivalence. Equivalently, $f: X \rightarrow Y$ is a weak equivalence if for all $H \subset G$ and all choices of base point $x_{0} \in X^{H}$ the induced map $\pi_{n}^{H}\left(X, x_{0}\right) \rightarrow \pi_{n}^{H}\left(Y, f\left(x_{0}\right)\right)$ is an isomorphism. Equipped with the weak equivalences, the category underlying $\mathcal{T}^{G}$ becomes a homotopical category. It can be completed to a topological model category in which a fibration is a map $X \rightarrow Y$ which for every $H \subset G$ is a Serre fibration on fixed points $X^{H} \rightarrow Y^{H}$. The smash product of $G$-spaces makes $\mathcal{T}^{G}$ into a symmetric monoidal category in the sense of Schwede-Shipley [75, Definition 3.1], and $\mathcal{I}_{G}$ into an enriched model category.

Every pointed $G$-space is weakly equivalent to a $G$-CW complex constructed inductively from the basepoint by attaching equivariant cells of the form $G / H \times D^{n}$ along maps from $G / H \times S^{n-1}$.

We will write both

$$
\text { ho } \mathcal{T}^{G}(X, Y) \text { and }[X, Y]^{G}
$$


for the set of maps from $X$ to $Y$ in the homotopy category of $\mathcal{T}^{G}$. When $X$ is cofibrant and $Y$ is fibrant this can be calculated as the set of homotopy classes of maps from $X$ to $Y$ in $\mathcal{T}^{G}$

$$
[X, Y]^{G}=\pi_{0} \mathcal{T}^{G}(X, Y)=\pi_{0}^{G} \underline{\mathcal{T}}_{G}(X, Y) .
$$

We will make frequent use of finite dimensional real orthogonal representations of $G$. To keep the terminology simple these will be referred to as representations of $G$.

An important role is played by the equivariant spheres $S^{V}$ arising as the one point compactification of representations $V$ of $G$. When $V$ is the trivial representation of dimension $n, S^{V}$ is just the $n$-sphere $S^{n}$ with the trivial $G$-action. We combine these two notations and write

$$
S^{V+n}=S^{V \oplus \mathbb{R}^{n}} .
$$

Associated to $S^{V}$ is the equivariant homotopy set

$$
\pi_{V}^{G} X=\left[S^{V}, X\right]^{G}
$$

defined to be the set of homotopy classes of $G$-equivariant maps from $S^{V}$ to $X$. The set $\pi_{V}^{G} X$ is a group if $\operatorname{dim} V>0$ and an abelian group if $\operatorname{dim} V^{G}>1$, where $V^{G}$ is the space of $G$-invariant vectors in $V$.

Also associated to the sphere $S^{V}$ one has the equivariant suspension $\Sigma^{V} X=$ $S^{V} \wedge X$ and the equivariant loop space $\Omega^{V} X=\underline{\mathcal{I}}_{G}\left(S^{V}, X\right)$.

Now suppose that $V_{1}$ and $V_{2}$ are two orthogonal representations of $G$ and that for each irreducible representation $U$ of $G$ occurring in $V_{1}$ one has

$$
\operatorname{dim} \operatorname{hom}^{G}\left(U, V_{2}\right) \geq \operatorname{dim} \operatorname{hom}^{G}\left(U, V_{1}\right) .
$$

Then one may choose an equivariant linear isometric embedding $t: V_{1} \rightarrow V_{2}$ and form

$$
\pi_{V_{2}-t\left(V_{1}\right)}^{G}(X)
$$

in which $V_{2}-t\left(V_{1}\right)$ denotes the orthogonal complement of the image of $V_{1}$ in $V_{2}$. The groups (2.2) form a local system over the Stiefel manifold $O\left(V_{1}, V_{2}\right)^{G}$ of equivariant linear isometric embeddings. If instead of (2.1) the one has $V_{2}>V_{1}$ in the sense of Definition 2.3 below, then the Stiefel manifold $O\left(V_{1}, V_{2}\right)$ is simply connected and one may define

$$
\pi_{V_{2}-V_{2}}^{G}(X)
$$

to be the group of global sections of this local system. For any $t \in O\left(V_{1}, V_{2}\right)^{G}$ the restriction map gives a canonical isomorphism $\pi_{V_{2}-V_{2}}^{G}(X) \rightarrow \pi_{V_{2}-t\left(V_{1}\right)}^{G}(X)$.

Definition 2.3. Let $V_{1}$ and $V_{2}$ be two non-zero $G$-representations. We write $V_{1}<$ $V_{2}$ if for every irreducible $G$-representation $U$,

$$
\operatorname{dim} \operatorname{hom}^{G}\left(U, V_{1}\right)<\operatorname{dim} \operatorname{hom}^{G}\left(U, V_{2}\right)-1 .
$$

This relation makes the set of $G$-representations into a (large) partially ordered set.

We will shortly ( $\$ 2.2 .4)$ be interested in the special case in which $V_{1}$ is a trivial representation of dimension $k$. As above we will write

$$
\pi_{V_{2}-k}^{G}(X)
$$


for this group. In this way, for any $n \in \mathbb{Z}$ there is a well-defined group

$$
\pi_{V+n}^{G}(X)
$$

provided $\operatorname{dim} V^{G} \geq-n+2$.

2.2. Equivariant stable homotopy theory. There is a choice to be made when stabilizing equivariant homotopy theory. If one only seeks that fibration sequences and cofibration sequences become weakly equivalent, then one stabilizes in the usual way, using suspensions by spheres with trivial $G$-action. But if one wants to have Spanier-Whitehead duals of finite $G$-CW complexes, one needs to stabilize with respect to the spheres $S^{V}$ where $V$ is a finite dimensional representation of $G$.

We will do equivariant stable homotopy theory in the category of equivariant orthogonal spectra, equipped with the stable weak equivalences. In order for this to be considered viable some properties must be established that guarantee computations made with equivariant orthogonal spectra ultimately reduce to computations in ho $\mathcal{T}^{G}$ in the expected manner. We therefore begin by discussing the equivariant Spanier-Whitehead category, and formulate six properties an equivariant stable homotopy should satisfy in order that it faithfully extend the Spanier-Whitehead category. These properties aren't enough for all of our purposes, so after establishing them for equivariant orthogonal spectra we turn the more refined structures (indexed products, coproducts, and smash products) that we require.

2.2.1. Spanier-Whitehead stabilization. The G-equivariant Spanier-Whitehead category $S \mathcal{W}^{G}$ is the category whose objects are finite pointed $G$-CW complexes and with maps

$$
\{X, Y\}^{G}=\underset{V}{\lim }\left[S^{V} \wedge X, S^{V} \wedge Y\right]^{G},
$$

in which the colimit is taken over the partially ordered set of $G$-representations. For an informative discussion of this category the reader is referred to [5].

There is a direct analogue $[5,84]$ of Spanier-Whitehead duality in $\mathcal{S} \mathcal{W}^{G}$, in which a finite based $G$-CW complex embedded in a representation sphere $S^{V}$ is " $V$-dual" to the unreduced suspension of its complement.

Example 2.4. Suppose that $X$ is a finite pointed $G$-set $B$. If there is an equivariant embedding $B \subset S^{V}$ (for instance when $V$ is the $G$-representation with basis $B$ ) the $V$-dual of $B$ works out to be $S^{V} \wedge B$.

If one wants finite $G$-CW complexes to have actual duals, in the sense of objects in a symmetric monoidal category, then one must enlarge the category $\mathcal{S W}^{G}$ by formally adding, for each finite $G$-CW complex $Y$ and each finite dimensional representation $V$ of $G$, an object $S^{-V} \wedge Y$ defined by

$$
\left\{X, S^{-V} \wedge Y\right\}^{G}=\left\{S^{V} \wedge X, Y\right\}^{G} .
$$

Since $\left\{S^{V} \wedge(-), Y\right\}^{G}$ is a functor on $\mathcal{S} \mathcal{W}^{G}$, this amounts to simply working in an enlargement of the Yoneda embedding of $\mathcal{S} \mathcal{W}^{G}$. One checks that for any $Z$, the map $Z \rightarrow S^{-V} \wedge S^{V} \wedge Z$ corresponding to the identity map of $S^{V} \wedge Z$ under (2.5) is an isomorphism, and that symmetric monoidal structure given by the smash product extends to this enlarged category. If $X$ and $Y$ are $V$-duals in $\mathcal{S} \mathcal{W}^{G}$, then $X$ and $S^{-V} \wedge Y$ are duals in the enlarged equivariant Spanier-Whitehead category.

Example 2.6. From Example 2.4, B is self-dual in the enlarged equivariant SpanierWhitehead category. 
As in the non-equivariant case, the equivariant Spanier-Whitehead category still suffers the defect that it is also not quite set up for doing stable homotopy theory. What one wants is a complete closed symmetric monoidal category $\mathcal{S}^{G}$ of G-equivariant spectra, equipped with the structure of a homotopical category (or even a Quillen model category), and related to $\mathcal{T}^{G}$ by a pair of adjoint (suspension spectrum and zero space) functors

$$
\Sigma^{\infty}: \mathcal{T}^{G} \leftrightarrows \mathcal{S}^{G}: \Omega^{\infty}
$$

In order to know that computations made in this category reduce in the expected manner to those in classical stable homotopy theory, one would like this data to satisfy

$\mathbf{S p}_{1}^{\mathbf{G}}$ The functors $\Sigma^{\infty}$ and $\Omega^{\infty}$ induce adjoint functors

$$
\mathbf{L} \Sigma^{\infty}: \text { ho } \mathcal{T}^{G} \leftrightarrows \operatorname{hoS}^{G}: \mathbf{R} \Omega^{\infty}
$$

on the homotopy categories.

$\mathbf{S p}_{2}^{\mathbf{G}}$ The symmetric monoidal structure on $\mathcal{S}^{G}$ induces a closed symmetric monoidal structure on the homotopy category ho $\mathcal{S}^{G}$ and the functor $\mathbf{L} \Sigma^{\infty}$ is symmetric monoidal.

$\mathbf{S p}_{\mathbf{3}}^{\mathbf{G}}$ The functor $\mathbf{L} \Sigma^{\infty}$ extends to a fully faithful, symmetric monoidal embedding of $\mathcal{S} \mathcal{W}^{G}$ into ho $\mathcal{S}^{G}$.

$\mathrm{Sp}_{4}^{\mathrm{G}}$ The objects $S^{V}$ are invertible in ho $\mathcal{S}^{G}$ under the smash product, so in particular the above embedding of $\mathcal{S} \mathcal{W}^{G}$ extends to an embedding of the extended Spanier-Whitehead category.

$\mathbf{S p}_{5}^{\mathrm{G}}$ Arbitrary coproducts (denoted $\vee$ ) exist in ho $\mathcal{S}^{G}$ and can be computed by the formation of wedges. If $\left\{X_{\alpha}\right\}$ is a collection of objects of $\mathcal{S}^{G}$ and $K$ is a finite $G$-CW complex, then the map

$$
\bigoplus_{\alpha} \operatorname{ho}^{G}\left(K, X_{\alpha}\right) \rightarrow \operatorname{hos}^{G}\left(K, \bigvee_{\alpha} X_{\alpha}\right)
$$

is an isomorphism.

$\mathbf{S p}_{6}^{\mathbf{G}}$ Up to weak equivalence every object $X$ is presentable in $\mathcal{S}^{G}$ as a homotopy colimit

$$
\cdots \rightarrow S^{-V_{n}} \wedge X_{V_{n}} \rightarrow S^{-V_{n+1}} \wedge X_{V_{n+1}} \rightarrow \cdots,
$$

in which $\left\{V_{n}\right\}$ is a fixed increasing sequence of representations eventually containing every finite dimensional representation of $G$, and each $X_{V_{n}}$ is weakly equivalent to an object of the form $\Sigma^{\infty} K_{V_{n}}$, with $K_{V_{n}}$ a $G$-CW complex.

These properties aren't meant to constitute a characterization of $\mathcal{S}^{G}$, though they nearly do. The first five insist that $\mathcal{S}^{G}$ not be too small, and the last that it not be too big. Combined they show that, any computation one wishes to make in ho $\mathcal{S}^{G}$ can, in principle, be reduced to a computation in $\mathcal{S} \mathcal{W}^{G}$.

In all of the common models, and in particular in equivariant orthogonal spectra, the presentation $\mathbf{S p}_{\mathbf{6}}^{\mathbf{G}}$ is functorial. We call this the canonical homotopy presentation. It is described in detail in $\S$ B.4.3. For many purposes one can ignore most of the technical details of equivariant spectra, and just think in terms of the canonical homotopy presentation. 
Finally, unless the emphasis is on foundations, we will drop the $\mathbf{L}$ and $\mathbf{R}$ and implicitly assume that all of the functors have been derived, unless otherwise specified.

2.2.2. Equivariant orthogonal spectra. An orthogonal $G$-spectrum consists of a collection of pointed $G$-spaces $X_{V}$ indexed by the finite dimensional orthogonal representations $V$ of $G$, an action of the orthogonal group $O(V)$ (of non-equivariant maps) on $X_{V}$, and for each (not necessarily $G$-equivariant) orthogonal inclusion $t: V \subset W$ a map $S^{W-t(V)} \wedge X_{V} \rightarrow X_{W}$, in which $W-t(V)$ denotes the orthogonal complement of the image of $V$ in $W$. These maps are required to be compatible with the actions of $G$ and $O(V)$. Maps of equivariant orthogonal spectra are defined in the evident manner. For a more careful and detailed description see A.2.4.

Depending on the context, we will refer to orthogonal $G$-spectra as "equivariant orthogonal spectra," "orthogonal spectra," "G-spectra," and sometimes just as "spectra".

As with $G$-spaces, there are two useful ways of making the collection of $G$-spectra into a category. There is the topological category $\mathcal{S}^{G}$ just described, and the $G$ equivariant topological category $\underline{\mathcal{S}}_{G}$ of equivariant orthogonal spectra and $G$-spaces of non-equivariant maps. Thus for equivariant orthogonal spectra $X$ and $Y$ there is an identification

$$
\mathcal{S}^{G}(X, Y)=\underline{\mathcal{S}}_{G}(X, Y)^{G} .
$$

We will use the abbreviated notation $\mathcal{S}$ to denote $\mathcal{S}^{G}$ when $G$ is the trivial group.

If $V$ and $W$ are two orthogonal representations of $G$ the same dimension, and $O(V, W)$ is the $G$-space of (not necessarily equivariant) orthogonal maps, then

$$
O(V, W)+\underset{O(V)}{\wedge} X_{V} \rightarrow X_{W}
$$

is a $G$-equivariant homeomorphism. In particular an orthogonal $G$-spectrum $X$ is determined by the $X_{V}$ with $V$ a trivial $G$-representation. This implies that the category $\mathcal{S}^{G}$ is equivalent to the category of objects in $\mathcal{S}$ equipped with a $G$-action (Proposition A.19).

Both $\mathcal{S}^{G}$ and $\underline{\mathcal{S}}_{G}$ are tensored and cotensored over $G$-spaces:

$$
\begin{gathered}
(X \wedge K)_{V}=X_{V} \wedge K \\
\left(X^{K}\right)_{V}=\left(X_{V}\right)^{K} .
\end{gathered}
$$

Both categories are complete and cocomplete.

Definition 2.7. The suspension and 0-space functors are defined by

$$
\begin{gathered}
\left(\Sigma^{\infty} K\right)_{V}=S^{V} \wedge K \\
\Omega^{\infty} X=X_{\{0\}}
\end{gathered}
$$

where $\{0\}$ is the zero vector space.

The suspension spectrum functor is left adjoint to the 0-space functor. One has $\Sigma^{\infty} K=S^{0} \wedge K$ and more generally $\Sigma^{\infty}(K \wedge L)=\left(\Sigma^{\infty} K\right) \wedge L$. The functors $\Sigma^{\infty}$ and $\Omega^{\infty}$ may be regarded as topological functors between $\mathcal{T}^{G}$ and $\mathcal{S}^{G}$ or as $\mathcal{T}^{G}$-enriched functors relating $\underline{\mathcal{T}}_{G}$ and $\underline{\mathcal{S}}_{G}$.

For each $G$-representation $V$ there is a $G$-spectrum $S^{-V}$ characterized by the existence of a functorial equivariant isomorphism

$$
\underline{\mathcal{S}}_{G}\left(S^{-V}, X\right) \approx X_{V}
$$


(see §A.2.4). By the enriched Yoneda Lemma, every equivariant orthogonal Gspectrum $X$ is functorially expressed as a reflexive coequalizer

$$
\bigvee_{V, W} S^{-W} \wedge \underline{S}_{G}\left(S^{-W}, S^{-V}\right) \wedge X_{V} \rightrightarrows \bigvee_{V} S^{-V} \wedge X_{V} \rightarrow X
$$

We call this the tautological presentation of $X$.

The category $\mathcal{S}^{G}$ is a closed symmetric monoidal category under the smash product operation. The tensor unit is the sphere spectrum $S^{0}$. There are canonical identifications

$$
S^{-V} \wedge S^{-W} \approx S^{-V \oplus W}
$$

and in fact the association

$$
V \mapsto S^{-V}
$$

is a symmetric monoidal functor from the category of finite dimensional representations of $G$ (and isomorphisms) to $\mathcal{S}^{G}$. Because of the tautological presentation, this actually determines the smash product functor (see §A.2.5).

Regarding the adjoint functors

$$
\Sigma^{\infty}: \mathcal{T}^{G} \leftrightarrows \mathcal{S}^{G}: \Omega^{\infty}
$$

the left adjoint $\Sigma^{\infty}$ is symmetric monoidal. We will usually drop the $\Sigma^{\infty}$ and either not distinguish in notation between the suspension spectrum of a $G$-space and the $G$-space itself, or use $S^{0} \wedge K$.

2.2.3. Change of group and indexed monoidal products. The fact that the category $\mathcal{S}^{G}$ is equivalent to the category of objects in $\mathcal{S}$ equipped with a $G$-action has an important and useful consequence. It means that if a construction involving spectra happens to produce something with a $G$-action, it defines a functor with values in $G$-spectra. For example, if $H \subset G$ is a subgroup, there is a restriction functor $i_{H}^{*}: \mathcal{S}^{G} \rightarrow \mathcal{S}^{H}$ given by simply restricting the action to $H$. This functor has both a left and a right adjoint. The left adjoint is given by

$$
X \mapsto G_{+} \stackrel{\wedge}{H} X
$$

and may be written as a "wedge"

$$
\bigvee_{i \in G / H} X_{i}
$$

where $X_{i}=\left(H_{i}\right)_{+}{ }_{H}^{\wedge} X$ with $H_{i} \subset G$ the coset indexed by $i$. Similarly, the right adjoint is given by the $H$-fixed points of the internal function spectrum from $G$ to $X$, and may be written as a kind of product

$$
\prod_{i \in H \backslash G} X^{i} \approx \prod_{i \in G / H} X_{i}
$$

where $X^{i}=\operatorname{hom}^{H}\left(H^{i}, X\right)$ and $H^{i}$ is the left $H$-coset with index $i$. The identification of the two expressions is made using the map $g \mapsto g^{-1}$. There is also an analogous construction involving the smash product

$$
N_{H}^{G} X=\bigwedge_{i \in G / H} X_{i}
$$

These are special cases of a more general construction. 
Suppose that $G$ is a finite group, and $J$ is a finite set on which $G$ acts. Write $\mathcal{B}_{J} G$ for the category with object set $J$, in which a map from $j$ to $j^{\prime}$ is an element $g \in G$ with $g \cdot j=j^{\prime}$. We abbreviate this to $\mathcal{B} G$ in case $J=$ pt. Given a functor

$$
X: \mathcal{B}_{J} G \rightarrow \mathcal{S}
$$

define the indexed wedge, indexed product and indexed smash product of $X$ to be

$$
\bigvee_{j \in J} X_{j}, \quad \prod_{j \in J} X_{j}, \text { and } \bigwedge_{j \in J} X_{j}
$$

respectively. The group $G$ acts naturally on the indexed wedge and indexed smash product and so they define functors from the category of $\mathcal{B}_{J} G$-diagrams of spectra to $\mathcal{S}^{G}$. For more details, see $\S$ A.3.2.

Suppose that $H$ is a subgroup of $G$ and $J=G / H$. In this case the inclusion $\mathcal{B}_{\{e\}} H \rightarrow \mathcal{B}_{J} G$ of the full subcategory containing the identity coset is an equivalence. The restriction functor and its left Kan extension therefore give an equivalence of the category of $\mathcal{B}_{J} G$-diagrams of spectra with $\mathcal{S}^{H}$. Under this equivalence, the indexed wedge works out to be the functor

$$
G_{+} \underset{H}{\wedge}(-) .
$$

The indexed smash product is the norm functor

$$
N_{H}^{G}: \mathcal{S}^{H} \rightarrow \mathcal{S}^{G}
$$

sending an $H$-spectrum $X$ to the $G$-spectrum

$$
\bigwedge_{j \in G / H} X_{j}
$$

Remark 2.10. When the context is clear, we will sometimes abbreviate the $N_{H}^{G}$ simply to $N$ in order to avoid clustering of symbols.

The norm distributes over wedges in much the same way as the iterated smash product. A precise statement of the general "distributive law" appears in §A.3.3.

The functor $N_{H}^{G}$ is symmetric monoidal, commutes with sifted colimits, and so filtered colimits and reflexive coequalizers (Proposition A.53). The fact that $V \mapsto S^{-V}$ is symmetric monoidal implies that

$$
N_{H}^{G} S^{-V}=S^{-\operatorname{ind}_{H}^{G} V},
$$

where $\operatorname{ind}_{H}^{G} V$ is the induced representation. From the definition, one also concludes that for a pointed $G$-space $T$,

$$
N_{H}^{G}\left(S^{-V} \wedge T\right)=S^{-\operatorname{ind}_{H}^{G} V} \wedge N_{H}^{G} T,
$$

where $N_{H}^{G} T$ is the analogous norm functor on spaces.

Th norm first appeared in group cohomology (Evens [23]), and is often referred to as the "Evens transfer" or the "norm transfer." The analogue in stable homotopy theory originates in Greenlees-May [27]. 
2.2.4. Stable weak equivalences. The inequality of Definition 2.3 gives the collection of finite dimensional orthogonal $G$-representations the structure of a (large) partially ordered set. When $V_{1}$ is the trivial representation of dimension $k$ the condition $V_{2}>V_{1}$ means that

$$
\operatorname{dim} V_{2}^{G}>k+1,
$$

and we will use instead the abbreviation $V_{2}>k$. Using (2.12) we extend this to all $k \in \mathbb{Z}$.

Suppose we are given $X \in \mathcal{S}^{G}, K \in \mathcal{T}^{G}$, and two representations $V_{1}<V_{2}$. Choose an equivariant isometric embedding $t: V_{1} \rightarrow V_{2}$ and let $W$ be the orthogonal complement of $t\left(V_{1}\right)$ in $V_{2}$. Define

$$
\left[S^{V_{1}} \wedge K, X_{V_{1}}\right]^{G} \rightarrow\left[S^{V_{2}} \wedge K, X_{V_{2}}\right]^{G}
$$

by using the identification $S^{W} \wedge S^{V_{1}} \approx S^{V_{2}}$ and the structure map $S^{W} \wedge X_{V_{1}} \rightarrow X_{V_{2}}$ to form the composite

$$
\left[S^{V_{1}} \wedge K, X_{V_{1}}\right]^{G} \rightarrow\left[S^{W} \wedge S^{V_{1}} \wedge K, S^{W} \wedge X_{V_{1}}\right]^{G} \rightarrow\left[S^{V_{2}} \wedge K, X_{V_{2}}\right]^{G} .
$$

This map depends only on the path component of $t$ in $O\left(V_{1}, V_{2}\right)^{G}$, so the condition $V_{1}<V_{2}$ implies that (2.13) is independent of the choice of $t$.

Definition 2.14. Let $X$ be a $G$-spectrum and $k \in \mathbb{Z}$. For $H \subset G$ the $H$-equivariant $k^{\text {th }}$ stable homotopy group of $X$ is the group

$$
\pi_{k}^{H} X=\underset{V>-k}{\lim _{V+k}} \pi_{V}^{H}
$$

in which the colimit is taken over the partial ordered set of orthogonal $G$-representations $V$ satisfying $V>-k$.

The poset of $G$-representations is a class, not a set, so one must check that the colimit (2.15) actually exists.

Definition 2.16. An increasing sequence $V_{n} \subset V_{n+1} \subset \cdots$ of finite dimensional representations of $G$ is exhausting if any finite dimensional representation $V$ of $G$ admits an equivariant embedding in some $V_{n}$.

Any exhausting sequence $\cdots \subset V_{n} \subset V_{n+1} \subset \cdots$ is final in the poset of $G$ representations, so the map

$$
\underset{n}{\lim } \pi_{V_{n}+k}^{H} X_{V_{n}} \rightarrow \underset{V>-k}{\lim _{V+k}} \pi_{V}^{H}
$$

is an isomorphism. This gives the existence of the colimit (2.15), and shows that $\pi_{k}^{H} X$ can be computed as

$$
\pi_{k}^{H} X=\underset{n}{\lim } \pi_{V_{n}+k}^{H} X_{V_{n}}
$$

in which $\cdots \subset V_{n} \subset V_{n+1} \subset \cdots$ is any choice of exhausting sequence.

Definition 2.17. A stable weak equivalence (or just weak equivalence, for short) is a map $X \rightarrow Y$ in $\mathcal{S}^{G}$ inducing an isomorphism of stable homotopy groups $\pi_{k}^{H}$ for all $k \in \mathbb{Z}$ and $H \subset G$. 
Equipped with the stable weak equivalences, the category $\mathcal{S}^{G}$ becomes a homotopical category in the sense of [21], and so both the homotopy category ho $\mathcal{S}^{G}$ and the functor $\mathcal{S}^{G} \rightarrow$ ho $\mathcal{S}^{G}$ are defined. As with $G$-spaces, we will often employ the notation

$$
[X, Y]^{G}
$$

for ho $\mathcal{S}^{G}(X, Y)$. See $\S$ B.1 for more on the theory of homotopical categories, and for an explanation of the notion of left $(\mathbf{L})$ and right $(\mathbf{R})$ derived functors appearing in the discussion below.

2.2.5. Properties $\mathbf{S p}_{1}^{\mathbf{G}}-\mathbf{S p}_{6}^{\mathbf{G}}$. We now describe how properties $\mathbf{S p}_{1}^{\mathbf{G}}-\mathbf{S p}_{6}^{\mathbf{G}}$ are verified, deferring most of the technical details to Appendix B. The first five properties assert things only about the homotopy category and, save the fact that the symmetric monoidal structure is closed, they can be established using only the language of homotopical categories.

For $\mathbf{S p}_{1}^{\mathbf{G}}$, one checks directly from the definition that the functor $\Sigma^{\infty}$ preserves weak equivalences between $G$-spaces with non-degenerate base points, so that $\mathbf{L} \Sigma^{\infty} X$ can be computed as $\Sigma^{\infty} X$ if $X$ has a non-degenerate base point, or as $\Sigma^{\infty} \tilde{X}$ in general, where $\tilde{X}$ is formed from $X$ by adding a whisker at the base point. The right derived functor $\mathbf{R} \Omega^{\infty}$ is given by choosing any exhausting sequence and forming

$$
\mathbf{R} \Omega^{\infty} X=\underset{\longrightarrow}{\operatorname{holim}} \Omega^{V_{n}} X_{V_{n}}
$$

(See Proposition B.24). Verifying that $\mathbf{L} \Sigma^{\infty}$ and $\mathbf{R} \Omega^{\infty}$ are adjoint functors makes use of formula (2.18) below.

Regarding the symmetric monoidal structure $\left(\mathbf{S p}_{2}^{\mathbf{G}}\right)$, the smash product is not known to preserve weak equivalences between all objects but it does so on the full subcategory of $\mathcal{S}^{G} \times \mathcal{S}^{G}$ consisting of pairs $(X, Y)$ for which one of $X$ or $Y$ is cellular in the sense that it constructed inductively, starting with $*$ and attaching cells of the form $G_{+} \wedge_{H} S^{-V} \wedge D_{+}^{m}$, with $V$ a representation of $H$. Every $G$-spectrum receives a functorial weak equivalence from a cellular object, so this is enough to induce a symmetric monoidal smash product on ho $\mathcal{S}^{G}$. See $\S$ B.3.7. The fact that the symmetric monoidal structure is closed is best understood in the context of model categories. See §B.4.2, and especially Corollary B.80.

For $\mathbf{S p}_{\mathbf{3}}^{\mathbf{G}}$, there is a useful formula for maps in ho $\mathcal{S}^{G}$ in good cases. Choose an exhausting sequence $\left\{V_{n}\right\}$. For $K$ a finite $G$-CW complex, $\ell \in \mathbb{Z}$, and any $Y \in \mathcal{S}^{G}$ the definition of stable weak equivalence and some elementary facts about homotopical categories lead to the formula (Proposition B.44)

$$
\operatorname{ho} \mathcal{S}^{G}\left(S^{\ell} \wedge K, Y\right)=\underset{n}{\lim }\left[S^{V_{n}+\ell} \wedge K, Y_{V_{n}}\right]^{G},
$$

Using this one easily checks that functor $K \mapsto S^{0} \wedge K$ extends to a symmetric monoidal functor $\mathcal{S} \mathcal{W}^{G} \rightarrow$ ho $\mathcal{S}^{G}$. A little more work gives the generalization (Proposition B.49)

$$
\operatorname{ho} \mathcal{S}^{G}\left(S^{-V} \wedge K, Y\right)=\underset{n}{\lim }\left[S^{V_{n}} \wedge K, Y_{V \oplus V_{n}}\right]^{G},
$$

in which $V$ a representation of $G$,

For any representations $V, W$ of $G$, and any $X \in \mathcal{S}^{G}$, the map

$$
S^{-V} \wedge S^{V} \wedge X \rightarrow X
$$


is a weak equivalence (Proposition B.30). This ultimately implies that $S^{V}$ is invertible in ho $\mathcal{S}^{G}$ (Corollary B.48). This establishes $\mathbf{S p}_{4}^{\mathbf{G}}$.

The fact that the formation of arbitrary wedges preserves weak equivalences gives the first part of Property $\mathbf{S p}_{5}^{\mathbf{G}}$ (Corollary B.23). The second part follows from (2.18).

The canonical homotopy presentation of $\mathbf{S p}_{6}^{\mathbf{G}}$ is constructed by choosing an exhausting sequence $V=\left\{V_{n}\right\}$ and letting $X_{n}$ be an equivariant CW approximation to $X_{V_{n}}$. Since it involves more than just the homotopy category, the construction is easier to describe with a model category structure in place. For the details see $\S$ B.4.3.

Indexed monoidal products have convenient homotopy properties in $\mathcal{S}^{G}$. The formation of indexed wedges is homotopical (Definition B.7), in the sense that it preserves weak equivalences. This means that it need not be derived. The same is true of the formation of finite indexed products. The map

$$
\bigvee_{\alpha} X_{\alpha} \rightarrow \prod_{\alpha} X_{\alpha}
$$

from a finite indexed wedge to a finite indexed product is always a weak equivalence. This means in particular that for $H \subset G$, the map from the left to the right adjoint of the restriction functor

$$
\mathcal{S}^{G} \rightarrow \mathcal{S}^{H}
$$

is always a weak equivalence. Thus for $X \in \mathcal{S}^{G}$ and $Y \in \mathcal{S}^{H}$ there are isomorphisms

$$
\left[X, G_{+} \hat{H}^{\wedge}\right]^{G} \approx\left[X, \prod_{i \in G / H} Y_{i}\right]^{G} \approx\left[i_{H}^{*} X, Y\right]^{H} .
$$

The composite is the Wirthmüller isomorphism. Because of it, the right adjoint to the restriction functor tends not to appear explicitly when discussing the homotopy category.

Up to weak equivalence, indexed smash products can be computed using cellular approximations. Combining this with the properties of the norm listed in $\S 2.2 .3$ leads to a useful description of $N_{H}^{G} X$ in terms of the canonical homotopy presentation

$$
N_{H}^{G} X=\underset{V_{n}}{\operatorname{holim}} S^{-\operatorname{ind}_{H}^{G} V_{n}} \wedge N_{H}^{G} X_{V_{n}} .
$$

Finally, note that the formula (2.18) also implies that for any $k \in \mathbb{Z}$

$$
\pi_{k}^{H} X \approx \operatorname{hoS}^{H}\left(S^{k}, X\right) \approx \operatorname{hoS}^{G}\left(G_{+}{ }_{H} S^{k}, X\right)
$$

where for $k>0 S^{-k}$ is defined to be $S^{-\mathbb{R}^{k}}$ with $\mathbb{R}^{k}$ the trivial representation.

2.2.6. The model structure. Not all of the functors one wishes to consider have convenient homotopy theoretic properties.

Example 2.21. For a $G$-spectrum $X$ let

$$
\operatorname{Sym}^{n} X=X^{\wedge n} / \Sigma_{n}
$$

be the orbit spectrum of the $n$-fold iterated smash product by the action of the symmetric group. The map

$$
S^{-1} \wedge S^{1} \rightarrow S^{0}
$$


is a weak equivalence. However, the induced map

$$
\operatorname{Sym}^{n}\left(S^{-1} \wedge S^{1}\right) \rightarrow \operatorname{Sym}^{n} S^{0}
$$

is not. The right side is $S^{0}$ since it is the tensor unit, while the left side works out (Proposition B.117) to be weakly equivalent to the suspension spectrum of classifying space for $G$-equivariant principal $\Sigma_{n}$-bundles, pointed by a disjoint basepoint.

In order to go further it is useful to refine the homotopical category structure on $\mathcal{S}^{G}$ to a model category. Let $\mathcal{A}_{\text {cof }}$ be the set of maps

$$
\mathcal{A}_{\text {cof }}=\left\{G_{+}{ }_{H}^{\wedge} S^{-V} \wedge S_{+}^{n-1} \rightarrow G_{+}{ }_{H}^{\wedge} S^{-V} \wedge D_{+}^{n}\right\}
$$

in which $H \subset G$ is a subgroup of $G$ and $V$ is a representation of $H$ containing a non-zero invariant vector. The set $\mathcal{A}_{\text {cof }}$ is the set of generating cofibrations in the positive complete model structure on $\mathcal{S}^{G}$. The weak equivalences are the stable weak equivalences and the fibrations are the maps having the right lifting property against the acyclic cofibrations. See $\S$ B.4.1 for more details.

It works out that the symmetric power construction is homotopical on the class of cofibrant objects in the positive complete model structure (Proposition B.113).

Remark 2.23. The condition that $V$ contains a non-zero invariant vector is the positivity condition. It is due to Jeff Smith, and arose first in the theory of symmetric spectra (see the paragraph following Corollary 0.6 in [54]). The choice is dictated by two requirements. One is that symmetric power construction sends weak equivalences between cofibrant objects to weak equivalences. This is the key point in showing that the forgetful functor from commutative algebras in $\mathcal{S}^{G}$ to $\mathcal{S}^{G}$ creates a model category structure on commutative algebras in $\mathcal{S}^{G}$ (Proposition B.130). The other is that the geometric fixed point functor (§B.10) preserves (acyclic) cofibrations. The first requirement could be met by replacing "positive" with $\operatorname{dim} V>0$. The second requires $\operatorname{dim} V^{G}>0$, once one is using a positive model structure on $\mathcal{S}$.

2.2.7. Virtual representation spheres and $R O(G)$-graded cohomology. Using the spectra $S^{-V_{1}}$ and the spaces $S^{V_{0}}$ one can associate a stable "sphere" to each virtual representation $V$ of $G$. To do so, one first represents $V$ as difference [ $\left.V_{0}\right]-\left[V_{1}\right]$ of representations, and then sets

$$
S^{V}=S^{-V_{1}} \wedge S^{V_{0}}
$$

If $\left(V_{0}, V_{1}\right)$ and $\left(W_{0}, W_{1}\right)$ are two pairs of orthogonal representations representing the same virtual representation

$$
V=\left[V_{0}\right]-\left[V_{1}\right]=\left[W_{0}\right]-\left[W_{1}\right] \in R O(G),
$$

then there is a representation $U$, and an equivariant orthogonal isomorphism

$$
W_{1} \oplus V_{0} \oplus U \approx V_{1} \oplus W_{0} \oplus U .
$$

A choice of such data gives weak equivalences

$$
\begin{aligned}
S^{-W_{1}} \wedge S^{W_{0}} \leftarrow S^{-W_{1} \oplus V_{0} \oplus U} \wedge S^{W_{0} \oplus V_{0} \oplus U} & \\
& \approx S^{-V_{1} \oplus W_{0} \oplus U} \wedge S^{W_{0} \oplus V_{0} \oplus U} \rightarrow S^{-V_{1}} \wedge S^{V_{0}}
\end{aligned}
$$

Thus, up to weak equivalence

$$
S^{V}=S^{-V_{1}} \wedge S^{V_{0}}
$$


depends only on $V$. However, the weak equivalence between the spheres arising from different choices depends on data not specified in the notation. This leads to some subtleties in grading equivariant stable homotopy groups over the real representation ring $R O(G)$. See [5, $\S 6]$ and [57, Chapter XIII]. The virtual representation spheres arising in this paper always occur as explicit differences of actual representations.

In the positive complete model structure, the spectrum $S^{-V_{1}} \wedge S^{V_{0}}$ will be cofibrant if and only if the dimension of the fixed point space $V_{1}^{G}$ is positive.

Definition 2.24. Suppose that $V$ is a virtual representation of $G$. A positive representative of $V$ consists of a pair of representations $\left(V_{0}, V_{1}\right)$ with $\operatorname{dim} V_{1}^{G}>0$ and for which

$$
V=\left[V_{0}\right]-\left[V_{1}\right] \in R O(G) .
$$

Associated to every subgroup $H \subset G$ and every representation $V \in R O(H)$ is the group

$$
\pi_{V}^{H}(X)=\left[S^{V}, X\right]^{H} .
$$

An equivariant cohomology theory is associated to every equivariant orthogonal spectrum $E$, by

$$
\begin{aligned}
& E^{k}(X)=\left[X, S^{k} \wedge E\right]^{G} \\
& E_{k}(X)=\left[S^{k}, E \wedge X\right]^{G}=\pi_{k}^{G}(E \wedge X) .
\end{aligned}
$$

There is also an $R O(G)$-graded version, defined by

$$
\begin{aligned}
& E^{V}(X)=\left[X, S^{V} \wedge E\right]^{G} \\
& E_{V}(X)=\left[S^{V}, E \wedge X\right]^{G}=\pi_{V}^{G}(E \wedge X) .
\end{aligned}
$$

\subsection{Multiplicative properties.}

\subsubsection{Commutative and associative algebras.}

Definition 2.25. An equivariant commutative algebra (or just commutative algebra) is a unital commutative monoid in $\mathcal{S}^{G}$ with respect to the smash product operation. An equivariant associative algebra (associative algebra) is a unital associative monoid with respect to the smash product.

There is a weaker "up to homotopy notion" that sometimes comes up.

Definition 2.26. An equivariant homotopy associative algebra (or just homotopy associative algebra) is an associative algebra in ho $\mathcal{S}^{G}$. An equivariant homotopy commutative algebra (or just homotopy commutative algebra) is a commutative algebra in ho $\mathcal{S}^{G}$.

The category of commutative algebras in $\mathcal{S}^{G}$ and spaces of equivariant multiplicative maps will be denoted $\mathbf{C o m m}{ }^{G}$. The $\mathcal{T}^{G}$-enriched category of $G$-equivariant commutative algebras and $G$-spaces of non-equivariant multiplicative maps will be denoted $\mathbf{C o m m}_{G}$. The categories $\mathbf{C o m m}{ }^{G}$ and $\mathbf{C o m m}_{G}$ are tensored and cotensored over $\mathcal{T}$ and $\mathcal{T}^{G}$ respectively. The tensor product of an equivariant commutative algebra $R$ and a $G$-space $T$ will be denoted

$$
R \otimes T
$$

to distinguish it from the smash product. By definition

$$
\operatorname{Comm}_{G}(R \otimes T, E)=\underline{\mathcal{I}}_{G}\left(T, \operatorname{Comm}_{G}(R, E)\right) .
$$


We make $\mathbf{C o m m}^{G}$ into a homotopical category by defining a map to be a weak equivalence if the underlying map of orthogonal $G$-spectra is. The free commutative algebra functor

$$
X \mapsto \operatorname{Sym}(X)=\bigvee_{n \geq 0} \operatorname{Sym}^{n} X,
$$

is left adjoint to the forgetful functor. It takes weak equivalences between cofibrant spectra to weak equivalences (Proposition B.113). This is the key point in showing that the forgetful functor

$$
\mathrm{Comm}^{G} \rightarrow \mathcal{S}^{G}
$$

creates a $(\mathcal{T}$-enriched $)$ model category from the positive complete model structure on $\mathcal{S}^{G}$ (Proposition B.130), and that

$$
\operatorname{Comm}_{G} \rightarrow \underline{\mathcal{S}}_{G}
$$

creates a $\mathcal{I}_{G}$-enriched model structure. For $H \subset G$ the forgetful functor $\mathbf{C o m m}^{G} \rightarrow$ Comm $^{H}$ and its left adjoint form a Quillen morphism. A similar set of results applies to associative algebras.

Modules over an equivariant commutative ring are defined in the evident way using the smash product. The category of left modules over $R$ and equivariant maps will be denoted $\mathcal{M}_{R}$. A map of $R$-modules is defined to be a weak equivalence if the underlying map of spectra is a weak equivalence. The adjoint "free module" and "forgetful" functors

$$
X \mapsto R \wedge X: \mathcal{S}^{G} \leftrightarrows \mathcal{M}_{R}: M \mapsto M
$$

create a model category structure on $\mathcal{M}_{R}$. It becomes an enriched symmetric monoidal model category under the operation

$$
M \underset{R}{\wedge} N
$$

where $M$ is regarded as a right $R$-module via

$$
M \wedge R \stackrel{\text { flip }}{\longrightarrow} R \wedge M \rightarrow M,
$$

and $M \underset{R}{\wedge} N$ is defined by the coequalizer diagram

$$
M \wedge R \wedge N \rightrightarrows M \wedge N \rightarrow M \underset{R}{\wedge} N
$$

There are also the related notions of $E_{\infty}$ and $A_{\infty}$ algebras. It can be shown that the categories of $E_{\infty}$ and commutative algebras are Quillen equivalent, as are those of $A_{\infty}$ and associative algebras.

2.3.2. Commutative algebras and indexed monoidal products. Because it is symmetric monoidal, the functor $N$ take commutative algebras to commutative algebras, and so induces a functor

$$
N=N_{H}^{G}: \mathbf{C o m m}^{H} \rightarrow \mathbf{C o m m}^{G} .
$$

The following result is proved in the Appendices, as Corollaries A.56 and B.134.

Proposition 2.27. The functor

$$
N: \mathbf{C o m m}^{H} \rightarrow \mathbf{C o m m}^{G} .
$$

is left adjoint to the restriction functor $i^{*}$. Together they form a Quillen morphism of model categories. 
Corollary 2.28. There is a natural isomorphism

$$
N_{H}^{G}\left(i_{H}^{*} R\right) \rightarrow R \otimes(G / H),
$$

under which the counit of the adjunction is identified with the map

$$
R \otimes(G / H) \rightarrow R \otimes(p t)
$$

given by the unique $G$-map $G / H \rightarrow p t$.

Proof: Since both $R \otimes(G / H)$ and the left adjoint to restriction corepresent the same functor, this follows from Proposition 2.27

A useful consequence Corollary 2.28 is that the group $N(H) / H$ of $G$-automorphisms of $G / H$ acts naturally on $N_{H}^{G}\left(i_{H}^{*} R\right)$. The result below is used in the main computational assertion of Proposition 5.50.

Corollary 2.29. For $\gamma \in N(H) / H$ the following diagram commutes:

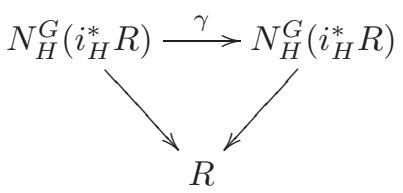

Proof: Immediate from Corollary 2.28.

At this point a serious technical issue arises. The spectra underlying commutative rings are almost never cofibrant. This means that there is no guarantee that the norm of a commutative ring has the correct homotopy type. The fact that it does is one of the main results of Appendix B. The following is a consequence of Proposition B.147.

Proposition 2.30. Suppose that $R$ is a cofibrant commutative $H$-algebra, and $\tilde{R} \rightarrow R$ is a cofibrant approximation of the underlying $H$-spectrum. If $\tilde{Z} \rightarrow Z$ is a weak equivalence of $G$-spectra then

$$
N_{H}^{G}(\tilde{R}) \wedge \tilde{Z} \rightarrow N_{H}^{G}(R) \wedge Z
$$

is a weak equivalence.

We refer to the property exhibited in Proposition 2.30 by saying that cofibrant commutative rings are very flat.

2.3.3. Other uses of the norm. There are several important constructions derived from the norm functor which also go by the name of "the norm."

Suppose that $R$ is a $G$-equivariant commutative ring spectrum, and $X$ is an $H$-spectrum for a subgroup $H \subset G$. Write

$$
R_{H}^{0}(X)=\left[X, i_{H}^{*} R\right]^{H} .
$$

There is a norm map

$$
N_{H}^{G}: R_{H}^{0}(X) \rightarrow R_{G}^{0}\left(N_{H}^{G} X\right)
$$

defined by sending an $H$-equivariant map $X \rightarrow R$ to the composite

$$
N_{H}^{G} X \rightarrow N_{H}^{G}\left(i_{H}^{*} R\right) \rightarrow R
$$

in which the second map is the counit of the restriction-norm adjunction. This is the norm map on equivariant spectrum cohomology, and is the form in which 
the norm is described in Greenlees-May [27]. For an explicit comparison with [27] see [11].

When $V$ is a representation of $H$ and $X=S^{V}$ the above gives a map

$$
N=N_{H}^{G}: \pi_{V}^{H} R \rightarrow \pi_{\text {ind } V}^{G} R
$$

in which ind $V$ is the induced representation which we call the norm map on the $R O(G)$-graded homotopy groups of commutative rings

Now suppose that $X$ is a pointed $G$-space. There is a norm map

$$
N_{H}^{G}: R_{H}^{0}(X) \rightarrow R_{G}^{0}(X)
$$

sending

$$
x \in R_{H}^{0}(X)=\left[S^{0} \wedge X, i_{H}^{*} R\right]^{H}
$$

to the composite

$$
S^{0} \wedge X \rightarrow S^{0} \wedge N(X) \approx N\left(S^{0} \wedge X\right) \rightarrow N\left(i_{H}^{*} R\right) \rightarrow R,
$$

in which the equivariant map of pointed $G$-spaces

$$
X \rightarrow N_{H}^{G}(X)
$$

is the "diagonal"

$$
X \rightarrow \prod_{j \in G / H} X_{j} \rightarrow \bigwedge_{j \in G / H} X_{j}
$$

whose $j^{\text {th }}$ component is the inverse to the isomorphism

$$
X_{j}=\left(H_{j}\right)_{+} \underset{H}{\wedge} X \rightarrow X
$$

given by the action map. That this is actually equivariant is probably most easily seen by making the identification

$$
X_{j} \approx \operatorname{hom}_{H}\left(H_{j}^{-1}, X\right)
$$

in which $H_{j}^{-1}$ denotes the left $H$-coset consisting of the inverses of the elements of $H_{j}$, and then writing

$$
\prod_{j \in G / H} X_{j} \approx \operatorname{hom}_{H}(G, X) .
$$

Under this identification, the "diagonal" map is the map

$$
X \rightarrow \operatorname{hom}_{H}(G, X)
$$

adjoint to the action map

$$
\underset{H}{\times} X \rightarrow X
$$

which is clearly equivariant.

One can combine these construction to define the norm on $R O(G)$-graded cohomology of a $G$-space $X$

$$
N_{H}^{G}: R_{H}^{V}(X) \rightarrow R_{G}^{\mathrm{ind} V}(X)
$$

sending

$$
S^{0} \wedge X \stackrel{a}{\rightarrow} S^{V} \wedge i_{H}^{*} R
$$

to the composite

$$
S^{0} \wedge X \rightarrow S^{0} \wedge N X \stackrel{N a}{\longrightarrow} S^{\text {ind } V} \wedge N i_{H}^{*} R \rightarrow S^{\text {ind } V} \wedge R .
$$


2.4. The method of twisted monoid rings. In this section we describe the method of twisted monoid rings. The basic constructions are categorical, and in $\S \S 2.4 .1-2.4 .2$ we do not make any homotopy theoretic considerations. In $\S 2.4 .3$ we take up the homotopy theoretic aspects of our constructions.

2.4.1. Twisted monoid rings. We start with a subgroup $H$ of $G$, and a positive representative $\left(V_{0}, V_{1}\right)$ of a virtual representation $V$ of $H$. Let

$$
S^{0}\left[S^{V}\right]=\bigvee_{k \geq 0}\left(S^{V}\right)^{\wedge k}
$$

be the free $H$-equivariant associative algebra generated by $S^{V}=S^{-V_{1}} \wedge S^{V_{0}}$, and

$$
\bar{x} \in \pi_{V}^{H} S^{0}\left[S^{V}\right]
$$

the homotopy class of the generating inclusion. When $|\bar{x}|=0$, the spectrum $S^{0}\left[S^{V}\right]$ is the monoid ring of the free monoid on one generator, and is in fact commutative. For general $\bar{x}$ it is the Thom spectrum of an associative monoid map from the free monoid on one generator to the classifying space for $H$-equivariant real vector bundles, hence a twisted monoid ring. It is not typically a commutative algebra, though the $R O(H)$-equivariant homotopy groups make it appear so, since $\pi_{\star}^{H} S^{0}\left[S^{V}\right]$ is a free module over $\pi_{\star}^{H} S^{0}$ with basis $\left\{1, \bar{x}, \bar{x}^{2}, \ldots\right\}$. It will be convenient to use the notation

$$
S^{0}[\bar{x}]=S^{0}\left[S^{V}\right]
$$

Using the norm functor we can form the $G$-equivariant twisted monoid ring

$$
N_{H}^{G}\left(S^{0}\left[S^{V}\right]\right)=S^{0}\left[G_{+}{ }_{H}^{\wedge} S^{V}\right] .
$$

This spectrum can also be described as a Thom spectrum over the free commutative monoid generated by $G / H$. Things will look cleaner, and better resemble the (polynomial) algebras we are modeling if we use the alternate notation

$$
S^{0}\left[G \cdot S^{V}\right] \text { and } S^{0}[G \cdot \bar{x}] .
$$

Though the symbol $H$ is omitted in this notation, it is still referenced. The representation $V$ is representation of $H$, and $\bar{x}$ is an $H$-equivariant map with domain $S^{V}$.

By smashing examples like these together we can make associative algebras that are twisted forms of free commutative monoid algebras over $S^{0}$, in which the group $G$ is allowed to act on the monoid. More explicitly, suppose we are given a sequence (possibly infinite) of subgroups $H_{i} \subset G$ and for each $i$ a positive representative $\left(\left(V_{i}\right)_{0},\left(V_{i}\right)_{1}\right)$ of a virtual representation $V_{i}$ of $H_{i}$. For each $i$ form

$$
S^{0}\left[G \cdot \bar{x}_{i}\right]
$$

as described above, smash the first $m$ together to make

$$
S^{0}\left[G \cdot \bar{x}_{1}, \ldots, G \cdot \bar{x}_{m}\right],
$$

and then pass to the colimit to construct the $G$-equivariant associative algebra

$$
T=S^{0}\left[G \cdot \bar{x}_{1}, G \cdot \bar{x}_{2}, \ldots\right] .
$$

The twisted monoid ring $T$ can also be described as a Thom spectrum over the free commutative monoid generated by the $G$-set

$$
J=\coprod_{i=1}^{\infty} G / H_{i} .
$$


By construction, it is an indexed smash product of an indexed wedge

$$
T=\bigwedge_{j \in J} \bigvee_{n=0}^{\infty} S^{n V(j)}
$$

where for $j=g H_{i}, V(j)$ is the virtual representation of $g H_{i} g^{-1}$ with positive representative

$$
V(j)=\left(V(j)_{0}, V(j)_{1}\right)=\left(g H_{i}\right) \underset{H_{i}}{\times}\left(\left(V_{i}\right)_{0},\left(V_{i}\right)_{1}\right)
$$

All of this can be done relative to an equivariant commutative algebra $R$ by defining

$$
R\left[G \cdot \bar{x}_{1}, G \cdot \bar{x}_{2}, \ldots\right]
$$

to be

$$
R \wedge S^{0}\left[G \cdot \bar{x}_{1}, G \cdot \bar{x}_{2}, \ldots\right] .
$$

Because they can fail to be commutative, these twisted monoid rings do not have all of the algebraic properties one might hope for. But it is possible to naturally construct all of the equivariant monomial ideals. Here is how.

Applied to (2.31) the distributive law of $\S$ A.3.3 gives an isomorphism of $T$ with the indexed wedge

$$
T=\bigvee_{f \in \mathbb{N}_{0}^{J}} S^{V_{f}}
$$

in which $f$ is running through the set of functions

$$
J \rightarrow \mathbb{N}_{0}=\{0,1,2, \ldots\}
$$

taking non-zero values on only finitely many elements (finitely supported functions). The group $G$ acts on the set $\mathbb{N}_{0}^{J}$ through its action on $J$, and $V_{f}$ is the virtual representation

$$
V_{f}=\sum_{j \in J} f(j) \cdot V(j)
$$

of the stabilizer $H_{f}$ of $f$, with the evident positive representation

$$
\left(\bigoplus_{j \in J} V(j)_{0}^{f(j)}, \bigoplus_{j \in J} V(j)_{1}^{f(j)}\right) .
$$

The $G$-set $\mathbb{N}_{0}^{J}$ is a commutative monoid under addition of functions, and the ring structure on $T$ is the indexed sum of the obvious isomorphisms

$$
S^{V_{f}} \wedge S^{V_{g}} \approx S^{V_{f} \oplus V_{g}} \approx S^{V_{f+g}} .
$$

Recall (for example from [15]) that an ideal in a commutative monoid $L$ is a subset $I \subset L$ with the property that $L+I \subset I$. Given a $G$-stable ideal $I \subset \mathbb{N}_{0}^{J}$ form the $G$-spectrum

$$
T_{I}=\bigvee_{f \in I} S^{V_{f}} .
$$

The formula for the multiplication in $T$ implies that $T_{I}$ is an equivariant sub bimodule of $T$, and that the association $I \mapsto T_{I}$ is an inclusion preserving function from the set of ideals in $\mathbb{N}_{0}^{J}$ to the set of sub-bimodules of $T$. For a more general and systematic discussion see $\S A .3 .6$. 
Example 2.32. The monomial ideal corresponding to the set $I$ of all non-zero elements of $\mathbb{N}_{0}^{J}$ is the augmentation ideal (up to homotopy it is the fiber of the map $\left.T \rightarrow S^{0}\right)$. It is convenient to denote this $T$ bimodule as $\left(G \cdot \bar{x}_{1}, G \cdot \bar{x}_{2}, \ldots\right)$. More generally, for an integer $n>0$ the set $n I=I+\cdots+I$ of $n$-fold sums of elements of $I$ is a monoid ideal. It corresponds to the monomial ideal given by the $n^{\text {th }}$ power of the augmentation ideal.

Example 2.33. Let $\operatorname{dim}: \mathbb{N}_{0}^{J} \rightarrow \mathbb{N}_{0}$ be the function given by

$$
\operatorname{dim} f=\operatorname{dim} V_{f}=\sum_{j \in J} f(j) \operatorname{dim} V_{j} .
$$

If for all $i, \operatorname{dim} V_{i}>0$, then the set $\{f \mid \operatorname{dim} f \geq d\}$ is a monoid ideal in $\mathbb{N}_{0}^{J}$ and corresponds to the monomial ideal $M \subset T$ consisting the wedge of spheres of dimension greater than $d$. The quotient bimodule $M_{d} / M_{d-1}$ can be identified with the indexed coproduct

$$
\bigvee_{\operatorname{dim} f=d} S^{V_{f}}
$$

on which $T$ is acting through the augmentation $T \rightarrow S^{0}$. These monomial ideals play an important role in the proof of the Slice Theorem in $\S 6$.

\subsubsection{The method of twisted monoid rings.}

Definition 2.34. Suppose that

$$
f_{i}: B_{i} \rightarrow R, \quad i=1, \ldots m
$$

are algebra maps from associative algebra $B_{i}$ to a commutative algebra $R$. The smash product of the $f_{i}$ is the algebra map

$$
\bigwedge^{m} f_{i}: \bigwedge^{m} B_{i} \rightarrow \bigwedge^{m} R \rightarrow R
$$

in which the right most map is the iterated multiplication. If $B$ is an $H$-equivariant associative algebra, and $f: B \rightarrow i_{H}^{*} R$ is an algebra map, we define the norm of $f$ to be the $G$-equivariant algebra map

$$
N_{H}^{G} B \rightarrow R
$$

given by

$$
N_{H}^{G} B \rightarrow N_{H}^{G}\left(i_{H}^{*} R\right) \rightarrow R
$$

in which the rightmost map is the counit of the adjunction described in Proposition 2.27 .

These constructions make it easy to map a twisted monoid ring to a commutative algebra. Suppose that $R$ is a fibrant $G$-equivariant commutative algebra, and we're given a sequence

$$
\bar{x}_{i} \in \pi_{V_{i}}^{H_{i}} R, \quad i=1,2, \ldots
$$

A choice of positive representative $\left(\left(V_{0}\right)_{i},\left(V_{1}\right)_{i}\right)$ of $V_{i}$ and a map

$$
S^{V_{i}} \rightarrow R
$$

representing $\bar{x}_{i}$ determines an associative algebra map

$$
S^{0}\left[\bar{x}_{i}\right] \rightarrow R .
$$


Applying the norm gives a $G$-equivariant associative algebra map

$$
S^{0}\left[G \cdot \bar{x}_{i}\right] \rightarrow R .
$$

By smashing these together we can make a sequence of equivariant algebra maps

$$
S^{0}\left[G \cdot \bar{x}_{1}, \ldots, G \cdot \bar{x}_{m}\right] \rightarrow R .
$$

Passing to the colimit gives an equivariant algebra map

$$
S^{0}\left[G \cdot \bar{x}_{1}, G \cdot \bar{x}_{2}, \ldots\right] \rightarrow R
$$

representing the sequence $\bar{x}_{i}$. We will refer to this process by saying that the map (2.35) is constructed by the method of twisted monoid rings. The whole construction can also be made relative to a commutative algebra $S$, leading to an $S$-algebra map

$$
S\left[G \cdot \bar{x}_{1}, G \cdot \bar{x}_{2}, \ldots\right] \rightarrow R
$$

when $R$ is a commutative $S$-algebra.

2.4.3. Quotient modules. One important construction in ordinary stable homotopy theory is the formation of the quotient of a module $M$ over a commutative algebra $R$ by the ideal generated by a sequence $\left\{x_{1}, x_{2}, \ldots\right\} \subset \pi_{*} R$. This is done by inductively forming the cofibration sequence of $R$-modules

$$
\Sigma^{\left|x_{n}\right|} M /\left(x_{1}, \ldots, x_{n-1}\right) \rightarrow M /\left(x_{1}, \ldots, x_{n-1}\right) \rightarrow M /\left(x_{1}, \ldots, x_{n}\right)
$$

and passing to the homotopy colimit in the end. There is an evident equivalence

$$
M /\left(x_{1}, \ldots\right) \approx M \wedge_{R} R /\left(x_{1}, \ldots\right)
$$

in case $M$ is a cofibrant $R$-module. The situation is slightly trickier in equivariant stable homotopy theory, where the group $G$ might act on the elements $x_{i}$, and prevent the inductive approach described above. The method of twisted monoid rings (\$2.4.1) can be used to get around this difficulty.

Suppose that $R$ is a fibrant equivariant commutative algebra, and that

$$
\bar{x}_{i} \in \pi_{V_{i}}^{H_{i}}(R) \quad i=1,2, \ldots
$$

is a sequence of equivariant homotopy classes. Using the method of twisted monoid rings, construct an associative $R$-algebra map

$$
T=R\left[G \cdot \bar{x}_{1}, G \cdot \bar{x}_{2}, \ldots\right] \rightarrow R .
$$

Using this map, we may regard an equivariant $R$-module $M$ as a $T$-module. In addition to (2.38) we will make use of the augmentation $\epsilon: T \rightarrow R$ sending the $\bar{x}_{i}$ to zero.

Definition 2.39. The quotient module $M /\left(G \cdot \bar{x}_{1}, \ldots\right)$ is the $R$-module

$$
M \stackrel{\mathrm{L}}{\stackrel{\mathrm{T}}{T}} R
$$

in which $T$ acts on $M$ through the map (2.38) and on $R$ through the augmentation.

The symbol $\wedge$ denotes derived smash product. By Proposition B.139 it can be computed by taking a cofibrant approximation in either variable. 
Let us check that this construction reduces to the usual one when the group acting is the trivial group and $M$ is a cofibrant $R$-module. For ease of notation, write

$$
\begin{aligned}
T & =R\left[x_{1}, \ldots\right] \\
T_{n} & =R\left[x_{1}, \ldots, x_{n}\right] .
\end{aligned}
$$

Using the isomorphism

$$
R\left[x_{1}, \ldots\right] \approx R\left[x_{1}, \ldots, x_{n}\right] \underset{R}{\wedge} R\left[x_{n+1}, \ldots\right]
$$

one can construct an associative algebra map

$$
T \rightarrow R\left[x_{n+1}, \ldots\right]
$$

by smashing the augmentation

$$
R\left[x_{1}, \ldots, x_{n}\right] \rightarrow R
$$

sending each $x_{i}$ to 0 , with the identity map of $R\left[x_{n+1}, \ldots\right]$. By construction, the evident map of $T$-algebras

$$
\lim _{\longrightarrow} R\left[x_{n+1}, \ldots\right] \rightarrow R
$$

is an isomorphism, and hence so is the map

$$
\underset{\longrightarrow}{\longrightarrow} M \underset{T}{\wedge} R\left[x_{n+1}, \ldots\right] \rightarrow M \underset{T}{\wedge} R
$$

In fact this isomorphism is also a derived equivalence. To see this construct a sequence

$$
\rightarrow \cdots N_{n+1} \rightarrow N_{n+2} \cdots
$$

of cofibrations of cofibrant left $T$-module approximations to

$$
\rightarrow \cdots R\left[x_{n+1}, \ldots\right] \rightarrow R\left[x_{n+2}, \ldots\right] \rightarrow \cdots .
$$

We have

$$
\pi_{*} \lim _{\longrightarrow} N_{k} \approx \lim _{\longrightarrow} \pi_{*} N_{k} \approx \lim _{\longrightarrow}\left(\pi_{*} R\right)\left[x_{k}, \ldots\right] \approx R
$$

from which one concludes that the map

$$
\stackrel{\lim }{\longrightarrow} N_{k} \rightarrow \underset{\lim }{\longrightarrow} R\left[x_{k}, \ldots\right]
$$

is a cofibrant approximation. It follows that

$$
M /\left(x_{1}, \ldots\right) \approx \operatorname{holim} M /\left(x_{1}, \ldots, x_{n}\right) .
$$

To compare $M /\left(x_{1}, \ldots, x_{n-1}\right)$ with $M /\left(x_{1}, \ldots, x_{n}\right)$ let $T_{n} \rightarrow R\left[x_{n}\right]$ be associative algebra map constructed from the isomorphism

$$
T_{n} \approx T_{n-1} \underset{R}{\wedge} R\left[x_{n}\right] .
$$

by smashing the augmentation of $T_{n-1}$ with the identity map of $R\left[x_{n}\right]$. We have

$$
M /\left(x_{1}, \ldots, x_{n-1}\right) \sim M \underset{T_{n-1}}{\wedge} R \approx M \underset{T_{n}}{\wedge} T_{n} \underset{T_{n-1}}{\wedge} R \approx M \underset{T_{n}}{\wedge} R\left[x_{n}\right] .
$$

By Proposition B.139 $M \underset{T_{n}}{\wedge} R\left[x_{n}\right]$ is a cofibrant $R\left[x_{n}\right]$-module. The cofibration sequence (2.37) is now constructed by applying the functor

$$
M /\left(x_{1}, \ldots, x_{n-1}\right) \underset{R\left[x_{n}\right]}{\wedge}(-) .
$$


to the pushout diagram of $R\left[x_{n}\right]$ bimodules

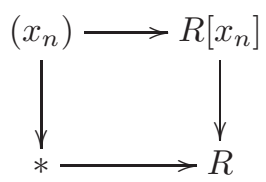

and appealing to Corollary B.140.

A similar discussion applies to the equivariant situation, giving

$$
M /\left(G \cdot \bar{x}_{1}, \ldots\right) \approx \lim M /\left(G \cdot \bar{x}_{1}, \ldots, G \cdot \bar{x}_{n}\right),
$$

a relation

$$
M /\left(G \cdot \bar{x}_{1}, \ldots, G \cdot \bar{x}_{n}\right) \approx M /\left(G \cdot \bar{x}_{1}, \ldots, G \cdot \bar{x}_{n-1}\right) \underset{R\left[G \cdot \bar{x}_{n}\right]}{\wedge} R,
$$

and a cofibration sequence

$\left(G \cdot \bar{x}_{n}\right) \cdot M /\left(G \cdot \bar{x}_{1}, \ldots, G \cdot \bar{x}_{n-1}\right) \rightarrow M /\left(G \cdot \bar{x}_{1}, \ldots, G \cdot \bar{x}_{n-1}\right) \rightarrow M /\left(G \cdot \bar{x}_{1}, \ldots, G \cdot \bar{x}_{n}\right)$, derived by applying the functor

$$
M /\left(G \cdot \bar{x}_{1}, \ldots, G \cdot \bar{x}_{n-1}\right) \underset{R\left[G \cdot \bar{x}_{n}\right]}{\wedge}(-)
$$

to

$$
\left(G \cdot \bar{x}_{n}\right) \rightarrow R\left[G \cdot \bar{x}_{n}\right] \rightarrow R .
$$

One can also easily deduce the equivalences

$$
R /\left(G \cdot \bar{x}_{1}, \ldots, G \cdot \bar{x}_{n}\right) \approx R /\left(G \cdot \bar{x}_{1}\right) \underset{R}{\wedge} \ldots \stackrel{\wedge}{\wedge} R /\left(G \cdot \bar{x}_{1}\right)
$$

and

$$
R /\left(G \cdot \bar{x}_{1}, \ldots\right) \approx \lim _{\longrightarrow} R /\left(G \cdot \bar{x}_{1}\right) \underset{R}{\wedge} \cdots \wedge_{R} R /\left(G \cdot \bar{x}_{n}\right) .
$$

These expressions play an important role in the proof Lemma 7.7, which is a key step in the proof the Reduction Theorem.

\subsection{Fixed points, isotropy separation and geometric fixed points.}

2.5.1. Fixed point spectra. The fixed point spectrum of a $G$-spectrum $X$ is defined to be the spectrum of $G$ fixed points in the underlying, non-equivariant spectrum $i_{0}^{*} X$. In other words it is given by

$$
X \mapsto\left(i_{0}^{*} X\right)^{G} .
$$

The notation $i_{0}^{*} X^{G}$ can get clumsy and we will usually abbreviate it to $X^{G}$.

The functor of fixed points has a left adjoint which sends $S^{-V} \wedge X_{V} \in \mathcal{S}$ to $S^{-V} \wedge X_{V} \in \mathcal{S}^{G}$, where in the latter expression $V$ is regarded as a representation of $G$ with trivial $G$-action and $X_{V}$ is regarded as a space with trivial $G$-action. It can be computed for general $X$ in terms of the tautological presentation

$$
\bigvee_{V, W} \mathscr{J}(V, W)_{+} \wedge S^{-W} \wedge X_{V} \rightrightarrows \bigvee_{V} S^{-V} \wedge X_{V} \rightarrow X
$$

for the trivial group (see (A.15)), once one observes that

$$
\mathscr{J}(V, W)=\mathscr{J}_{G}(V, W)
$$

when $V$ and $W$ have trivial $G$-action. 
Under the equivalence between $\mathcal{S}^{G}$ and the category of objects in $\mathcal{S}$ equipped with a $G$-action, the fixed point spectrum functor is formed by passing to objectwise fixed points, and its left adjoint is given by regarding a non-equivariant spectrum as a $G$-object with trivial $G$-action.

The fixed point functor and its left adjoint form a Quillen morphism in the positive complete model structures. Neither the fixed point functor nor its left adjoint is homotopical and so both need to be derived. As one can easily check from the definition, if $X$ is fibrant (or more generally has the property that for some exhausting sequence $\left\{V_{n}\right\}$, the map $X_{V_{n}} \rightarrow \Omega^{V_{n+1}-V_{n}} X_{V_{n+1}}$ is a weak equivalence) there is an isomorphism

$$
\pi_{*}\left(X^{G}\right) \approx \pi_{*}^{G} X
$$

The (derived) fixed point functor on spectra doesn't always have the properties one might be led to expect by analogy with spaces. For example even though the composition of the fixed point functor with its left adjoint is the identity, the composition of the derived functors is not. The derived fixed point functor does not generally commute with smash products, or with the formation of suspension spectra.

2.5.2. Isotropy separation and geometric fixed points. A standard approach to getting at the equivariant homotopy type of a $G$-spectrum $X$ is to nest $X$ between two pieces, one an aggregate of information about the spectra $i_{H}^{*} X$ for all proper subgroups $H \subset G$, and the other a localization of $X$ at a "purely $G$ " part. This is the isotropy separation sequence of $X$.

More formally, let $\mathcal{P}$ denote the family of proper subgroups of $G$, and $E \mathcal{P}$ the "classifying space" for $\mathcal{P}$, characterized up to equivariant weak equivalence by the property that the space of fixed points $E \mathcal{P}^{G}$ is empty, while for any proper $H \subset G$, $E \mathcal{P}^{H}$ is weakly contractible. For convenience we will assume that $E \mathcal{P}$ has been chosen to be a $G$-CW complex. Such a model can be constructed as the join of infinitely many copies of $G / H$ with $H$ ranging through the proper subgroups of $G$. It can also be constructed as the unit sphere in the sum of infinitely many copies of the reduced regular representation of $G$. Any $G$-CW complex $E \mathcal{P}$ admits an equivariant cell decomposition into cells of the form $(G / H)_{+} \wedge D_{+}^{n}$ with $H$ a proper subgroup of $G$.

Let $\tilde{E} \mathcal{P}$ be the mapping cone of $E \mathcal{P} \rightarrow$ pt, with the cone point taken as base point. The $G$-CW complex $\tilde{E} \mathcal{P}$ is characterized up to equivariant homotopy equivalence by the property

$$
(\tilde{E} \mathcal{P})^{H} \sim \begin{cases}S^{0} & H=G \\ * & H \neq G .\end{cases}
$$

The important isotropy separation sequence is constructed by smashing a $G$ spectrum $X$ with the defining cofibration sequence for $\tilde{E} \mathcal{P}$

$$
E \mathcal{P}_{+} \wedge X \rightarrow X \rightarrow \tilde{E} \mathcal{P} \wedge X
$$

The term on the left can be described in terms of the action of proper subgroups $H \subset G$ on $X$. The homotopy type of the term on the right is determined by its right derived fixed point spectrum

$$
\Phi^{G}(X)=\left((\tilde{E} \mathcal{P} \wedge X)_{f}\right)^{G},
$$


in which the subscript $f$ indicates a functorial fibrant replacement. The functor $\Phi^{G}(X)$ is the geometric fixed point functor and has many remarkable properties.

Proposition 2.45. i) The functor $\Phi^{G}$ sends weak equivalences to weak equivalences.

ii) The functor $\Phi^{G}$ commutes with filtered homotopy colimits.

iii) For a $G$-space $A$ and a representation $V$ of $G$ there is a weak equivalence $\Phi^{G}\left(S^{-V} \wedge A\right) \approx S^{-V^{G}} \wedge A^{G}$ where $V^{G} \subset V$ is the subspace of $G$-invariant vectors.

iv) For $G$-spectra $X$ and $Y$ the spectra

$$
\Phi^{G}(X \wedge Y) \quad \text { and } \quad \Phi^{G}(X) \wedge \Phi^{G}(Y) .
$$

are related by a natural chain of weak equivalences.

Remark 2.46. Note that in terms of the canonical homotopy presentation

$$
X \approx \underset{V}{\operatorname{holim}} S^{-V} \wedge X_{V}
$$

properties i)-iii) of Proposition 2.45 imply that

$$
\Phi^{G} X \approx \underset{V}{\operatorname{holim}} S^{-V^{G}} \wedge X_{V}^{G}
$$

Sketch of proof: The first assertion follows from the fact that smashing with $\tilde{E} \mathcal{P}$ is homotopical (§B.3.7), so need not be derived, and that the fixed point functor is homotopical on the full subcategory of fibrant objects. The second is straightforward. Part iii) is Corollary B.185. By the remark above, the canonical homotopy presentation reduces part iv) to the case $X=S^{-V} \wedge A, Y=S^{-W} \wedge B$, with $A$ and $B G$-CW complexes. One easily checks the assertion in this case using part iii).

Remark 2.48. When $G=C_{2^{n}}$, the space $E \mathcal{P}$ is the space $E C_{2}$ with $G$ acting through the epimorphism $G \rightarrow C_{2}$. Taking $S^{\infty}$ with the antipodal action as a model of $E C_{2}$, this leads to an identification

$$
\tilde{E} \mathcal{P} \approx \lim _{n \rightarrow \infty} S^{n \sigma},
$$

in which $S^{n \sigma}$ denotes the one point compactification of the direct sum of $n$ copies of the real sign representation of $G$.

Remark 2.49. The isotropy separation sequence often leads to the situation of needing to show that a map $X \rightarrow Y$ of cofibrant $G$-spectra induces a weak equivalence

$$
\tilde{E} \mathcal{P} \wedge X \rightarrow \tilde{E} \mathcal{P} \wedge Y \text {. }
$$

Since for every proper $H \subset G, \pi_{*}^{H} \tilde{E} \mathcal{P} \wedge X=\pi_{*}^{H} \tilde{E} \mathcal{P} \wedge Y=0$, this is equivalent to showing that the map of geometric fixed point spectra $\Phi^{G} X \rightarrow \Phi^{G} Y$ is a weak equivalence.

Remark 2.50. Since for every proper $H \subset G, \pi_{*}^{H} \tilde{E} \mathcal{P} \wedge X=0$, it is also true that

$$
[T, \tilde{E} \mathcal{P} \wedge X]_{*}^{G}=0
$$


for every $G$-CW spectrum $T$ built entirely from $G$-cells of the form $G_{+} \wedge_{H} D^{n}$ with $H$ a proper subgroup of $G$. Similarly, if $T$ is gotten from $T_{0}$ by attaching $G$-cells induced from proper subgroups, then the restriction map

$$
[T, \tilde{E} \mathcal{P} \wedge X]_{*}^{G} \rightarrow\left[T_{0}, \tilde{E} \mathcal{P} \wedge X\right]_{*}^{G}
$$

is an isomorphism. This holds, for example, if $T$ is the suspension spectrum of a $G$-CW complex, and $T_{0} \subset T$ is the subcomplex of $G$-fixed points.

Remark 2.51. For a subgroup $H \subset G$ and a $G$-spectrum $X$, it will be convenient to use the abbreviation

$$
\Phi^{H} X
$$

for the more correct $\Phi^{H} i_{H}^{*} X$. This situation comes up in our proof of the "homotopy fixed point" property of Theorem 10.8, where the more compound notation becomes a little unwieldy.

We end this section with a simple result whose proof illustrates a typical use of the geometric fixed point spectra.

Proposition 2.52. Suppose that $X$ is a $G$-spectrum with the property that for all $H \subset G$, the geometric fixed point spectrum $\Phi^{H} X$ is contractible. Then $X$ is contractible as a G-spectrum.

Proof: By induction on $|G|$ we may assume that for proper $H \subset G$, the spectrum $i_{H}^{*} X$ is contractible. Since both $G_{+}{ }_{H}(-)$ and the formation of mapping cones are homotopical it follows that $T \wedge X$ is contractible for any $G$-CW complex built entirely from cells of the form $G_{+}{ }_{H} D^{n}$ with $H \subset G$ proper. This applies in particular to $T=E \mathcal{P}_{+}$. The isotropy separation sequence then shows that

$$
X \rightarrow \tilde{E} \mathcal{P} \wedge X
$$

is a weak equivalence. But Remark 2.49 and our assumption that $\Phi^{G} X$ is contractible imply that $\tilde{E} \mathcal{P} \wedge X$ is contractible.

2.5.3. Monoidal geometric fixed points. For some purposes it is useful to have a version of the geometric fixed point functor which is lax symmetric monoidal. For example, such a functor automatically takes (commutative) algebras to (commutative) algebras. The geometric fixed point functor defined in [53, §V.4] has this property. We denote it $\Phi_{M}^{G}$ and refer to it as the monoidal geometric fixed point functor in order to distinguish it from $\Phi^{G}$. The following is a compendium of results from $[53, \S \mathrm{V} .4]$. The construction and proofs are described in $\S \mathrm{B} .10$.

Proposition 2.53. The monoidal geometric fixed point functor has the following properties:

i) It preserves acyclic cofibrations.

ii) It is lax symmetric monoidal.

iii) If $X$ and $Y$ are cofibrant, the map

$$
\Phi_{M}^{G}(X) \wedge \Phi_{M}^{G}(Y) \rightarrow \Phi_{M}^{G}(X \wedge Y)
$$

is an isomorphism.

iv) It commutes with cobase change along a closed inclusion. 
v) It commutes with directed colimits.

Property iii) implies that $\Phi_{M}^{G}$ is weakly symmetric monoidal in the sense of the definition below.

Definition 2.54 (Schwede-Shipley [75]). A functor $F: \mathcal{C} \rightarrow \mathcal{D}$ between (symmetric) monoidal model categories is weakly (symmetric) monoidal if it is lax (symmetric) monoidal, and the map

$$
F(X) \wedge F(Y) \rightarrow F(X \wedge Y)
$$

is a weak equivalence when $X$ and $Y$ are cofibrant.

The next result is [53, Proposition V.4.17], and is discussed in more detail as Proposition B.201.

Proposition 2.55. The left derived functor of $\Phi_{M}^{G}$ is $\Phi^{G}$. More specifically, there are natural transformations

$$
\Phi^{G}(X) \rightarrow \tilde{\Phi}_{M}^{G}(X) \stackrel{\sim}{\longleftarrow} \Phi_{M}^{G}(X)
$$

in which the rightmost arrow is a always weak equivalence and the leftmost arrow is a weak equivalence when $X$ is cofibrant.

Because $\Phi^{G}$ is lax monoidal, it determines functors

$$
\begin{aligned}
& \Phi_{M}^{G}: \mathbf{A l g}^{G} \rightarrow \mathbf{A l g} \\
& \Phi_{M}^{G}: \mathbf{C o m m}^{G} \rightarrow \mathbf{C o m m},
\end{aligned}
$$

and for each associative algebra $R$ a functor

$$
\Phi_{M}^{G}: \mathcal{M}_{R} \rightarrow \mathcal{M}_{\Phi_{M}^{G} R} .
$$

In addition, if $R$ is an associative algebra, $M$ a right $R$-module and $N$ a left $R$ module there is a natural map

$$
\Phi_{M}^{G}(M \underset{R}{\wedge} N) \rightarrow \Phi_{M}^{G} M \underset{\Phi_{M}^{G} R}{\wedge} \Phi_{M}^{G} N .
$$

The argument for [53, Proposition V.4.7] shows that (2.56) is a weak equivalence (in fact an isomorphism) if $M$ and $N$ are cofibrant and $R$ is "cellular." See Proposition B.203. (Recently, Blumberg and Mandell [10, Appendix A] have shown that one need only require one of $M$ or $N$ to be cofibrant in order to guarantee that this map is an isomorphism.)

While these properties if $\Phi_{M}^{G}$ are very convenient, they must be used with caution. The value $\Phi_{M}^{G}(X)$ is only guaranteed to have the "correct" homotopy type on cofibrant objects. The spectrum underlying a commutative algebra is rarely known to be cofibrant, making the monoidal geometric fixed point functor difficult to use in that context. The situation is a little better with associative algebras. The weak equivalence (2.56) leads to an expression for the geometric fixed point spectrum of a quotient module which we will use in $\$ 7.3$. In order to do so, we need criteria guaranteeing that the monoidal geometric fixed point functor realizes the correct homotopy type. Such criteria are described in $§$ B.10.4. 
2.5.4. Geometric fixed points and the norm. The geometric fixed point construction interacts well with the norm. Suppose $H \subset G$ is a subgroup, and that $X$ is an $H$ spectrum. The following result is proved as Proposition B.209. Our original version merely concluded that the transformation in question was a weak equivalence on cofibrant objects. Andrew Blumberg and Mike Mandell pointed out that it is in fact an isomorphism on cofibrant objects, and at their request we have included a proof of the stronger statement.

Proposition 2.57. There is a natural map

$$
\Phi_{M}^{H} X \rightarrow \Phi_{M}^{G}\left(N_{H}^{G} X\right)
$$

which is an isomorphism, hence a weak equivalence, on cofibrant objects.

Because of Proposition 2.55 and the fact that the norm preserves cofibrant objects (Proposition B.89), the above result gives a natural zig-zag of weak equivalences relating $\Phi^{H}(X)$ and $\Phi^{G}\left(N_{H}^{G} X\right)$ when $X$ is cofibrant. In fact there is a natural zig-zag of maps

$$
\Phi^{H} X \leftrightarrow \Phi^{G}\left(N_{H}^{G} X\right)
$$

which is a weak equivalence not only for cofibrant $X$, but for suspension spectra of cofibrant $G$-spaces and for the spectra underlying cofibrant commutative rings. The actual statement is somewhat technical, and is one of the main results of Appendix B. The condition is described in the statement of Proposition B.213. See also Remarks B.215 and B.216.

Corollary 2.58. For the spectra satisfying the condition of Proposition B.213, the composite functor

$$
\Phi^{G} \circ N_{H}^{G}: \mathcal{S}^{H} \rightarrow \mathcal{S}
$$

preserves, up to weak equivalence, wedges, directed colimits along closed inclusions and cofiber sequences.

Proof: The properties obviously hold for $\Phi^{H}$.

There is another useful result describing the interaction of the geometric fixed point functor with the norm map in $R O(G)$-graded cohomology described in $\S 2.3 .3$. Suppose that $R$ is a $G$-equivariant commutative algebra, $X$ is a $G$-space, and $V \in$ $R O(H)$ a virtual real representation of a subgroup $H \subset G$. In this situation one can compose the norm

$$
N: R_{H}^{V}(X) \rightarrow R_{G}^{\text {ind } V}(X)
$$

with the geometric fixed point map

$$
\Phi^{G}: R_{G}^{\text {ind } V}(X) \rightarrow\left(\Phi^{G} R\right)^{V^{H}}\left(X^{G}\right),
$$

where $V^{H} \subset V$ is the subspace of $H$-fixed vectors, and $X^{G}$ is the space of $G$-fixed points in $X$.

Proposition 2.59. The composite

$$
\Phi^{G} \circ N: R_{H}^{V}(X) \rightarrow\left(\Phi^{G} R\right)^{V^{H}}\left(X^{G}\right)
$$

is a ring homomorphism. 
Proof: Multiplicativity is a consequence of the fact that both the norm and the geometric fixed point functors are weakly monoidal. Additivity follows from the fact that the composition $\Phi^{G} \circ N$ preserves wedges (Corollary 2.58).

\section{MACKEY FUNCTORS, HOMOLOGY AND HOMOTOPY}

3.1. Mackey functors. In equivariant homotopy theory, the role of "abelian group" is played by the notion of a Mackey functor (Dress [18]). The following is the summary of Dress' definition as it appears in Greenlees-May [26].

Definition 3.1 (Dress [18]). A Mackey functor consists of a pair $\underline{M}=\left(\underline{M}_{*}, \underline{M}^{*}\right)$ of functors from the category of finite $G$-sets to the category of abelian groups. The two functors have the same object function (denoted $\underline{M}$ ) and take disjoint unions to direct sums. The functor $\underline{M}_{*}$ is covariant, while $\underline{M}^{*}$ is contravariant, and together they take a pullback diagram of finite $G$-sets

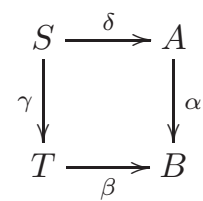

to a commutative square

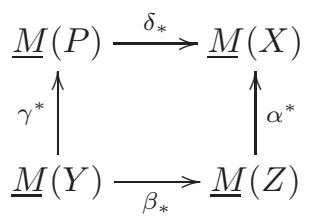

where $\alpha^{*}=\underline{M}^{*}(\alpha), \beta_{*}=\underline{M}_{*}(\beta)$, etc.

The contravariant maps $\underline{M}^{*}(\alpha)$ are called the restriction maps, and the covariant maps $\underline{M}_{*}(\beta)$, the transfer maps.

A Mackey functor can also be defined as a contravariant additive functor from the full subcategory of $\mathcal{S}^{G}$ consisting of the suspension spectra $\Sigma^{\infty} B_{+}$of finite $G$ sets $B$. It is a theorem of tom Dieck that these definitions are equivalent. See [26, $\S 5]$.

The equivariant homotopy groups of a $G$-spectrum $X$ are naturally part of the Mackey functor $\underline{\pi}_{n} X$ defined by

$$
\begin{aligned}
& \left(\underline{\pi}_{n} X\right)^{*}(B)=\left[S^{n} \wedge B_{+}, X\right]^{G} \\
& \left(\underline{\pi}_{n} X\right)_{*}(B)=\left[S^{n}, X \wedge B_{+}\right]^{G} .
\end{aligned}
$$

The identification of the two object functors

$$
\left[S^{n} \wedge B_{+}, X\right]^{G} \approx\left[S^{n}, X \wedge B_{+}\right]^{G}
$$

comes from the self-duality of finite $G$-sets (Example 2.6). For $B=G / H$ one has

$$
\underline{\pi}_{n} X(B)=\pi_{n}^{H} X \text {. }
$$

The Mackey functor $\underline{\pi}_{0} S^{0}$ is the Burnside ring Mackey functor $\underline{A}$. It is the free Mackey functor on one generator. For a $G$-set $B$, the value $\underline{A}(B)$ is the group completion of the monoid of isomorphism classes of finite $G$-sets $T \rightarrow B$ over $B$ under disjoint union. The restriction maps are given by pullback, and the transfer 
maps by composition. The group $\underline{A}(G / H)$ works out to be isomorphic to the abelian group underlying the Burnside ring of finite $H$-sets.

Just as every abelian group can occur as a stable homotopy group, every Mackey functor $\underline{M}$ can occur as an equivariant stable homotopy group. In fact associated to each Mackey functor $\underline{M}$ is an equivariant Eilenberg-Mac Lane spectrum $H \underline{M}$, characterized by the property

$$
\underline{\pi}_{n} H \underline{M}= \begin{cases}\underline{M} & n=0 \\ 0 & n \neq 0 .\end{cases}
$$

See $[26, \S 5]$ or $[47]$.

The homology and cohomology groups of a $G$-spectrum $X$ with coefficients in $\underline{M}$ are defined by

$$
\begin{aligned}
H_{k}^{G}(X ; \underline{M}) & =\pi_{k}^{G} H \underline{M} \wedge X \\
H_{G}^{k}(X ; \underline{M}) & =\left[X, \Sigma^{k} H \underline{M}\right]^{G} .
\end{aligned}
$$

For a pointed $G$-space $Y$ one defines

$$
\begin{aligned}
H_{n}^{G}(Y ; \underline{M}) & =H_{n}^{G}\left(\Sigma^{\infty} Y ; \underline{M}\right) \\
H_{G}^{n}(Y ; \underline{M}) & =H_{G}^{n}\left(\Sigma^{\infty} Y ; \underline{M}\right) .
\end{aligned}
$$

We emphasize that the equivariant (co)homology groups of pointed $G$-spaces $Y$ we consider will always be reduced (co)homology groups.

We will have need to consider the ordinary, non-equivariant homology and cohomology groups of the spectrum $i_{0}^{*} X$ underlying a $G$-spectrum $X$. It will be convenient to employ the shorthand notation

$$
\begin{aligned}
& H_{n}^{u}(X ; \mathbb{Z})=H_{n}\left(i_{0}^{*} X ; \mathbb{Z}\right) \\
& H_{u}^{n}(X ; \mathbb{Z})=H^{n}\left(i_{0}^{*} X ; \mathbb{Z}\right)
\end{aligned}
$$

for these groups.

3.2. Constant and permutation Mackey functors. The constant Mackey functor $\underline{\mathbb{Z}}$ is the functor represented on the category of finite $G$-sets by the abelian group $\mathbb{Z}$ with trivial $G$-action. The value of $\underline{\mathbb{Z}}$ on a finite $G$-set $B$ is the group of functions

$$
\underline{\mathbb{Z}}(B)=\operatorname{hom}^{G}(B, \mathbb{Z})=\operatorname{hom}(B / G, \mathbb{Z}) .
$$

The restriction maps are given by precomposition, and the transfer maps by summing over the fibers. For $K \subset H \subset G$, the transfer map associated by $\underline{\mathbb{Z}}$ to

$$
G / K \rightarrow G / H
$$

is the map $\mathbb{Z} \rightarrow \mathbb{Z}$ given by multiplication by the index of $K$ in $H$.

Definition 3.2. Suppose that $S$ is a $G$-set, and write $\mathbb{Z}\{S\}$ for the free abelian group generated by $S$, and $\mathbb{Z}^{S}$ for the ring of functions $S$ to $\mathbb{Z}$. The permutation Mackey functor $\underline{\mathbb{Z}}\{S\}$ is the Mackey functor

$$
\underline{\mathbb{Z}}\{S\}(B)=\operatorname{hom}^{G}(B, \mathbb{Z}\{S\}),
$$

whose restriction maps are given by precomposition and transfer maps by summing over the fibers. 
The permutation Mackey functor $\mathbb{Z}\{S\}$ is naturally isomorphic to the Mackey functor $\underline{\pi}_{0} H \underline{\mathbb{Z}} \wedge S_{+}$. To see this note that restricting to underlying non-equivariant spectra gives a map

$$
\underline{\pi}_{0} H \underline{\mathbb{Z}} \wedge S_{+}(B)=\left[B_{+}, H \underline{\mathbb{Z}} \wedge S_{+}\right]^{G} \rightarrow\left[B_{+}, H \mathbb{Z} \wedge S_{+}\right]
$$

whose image lies in the $G$-invariant part. Since

$$
\left[B_{+}, H \underline{\mathbb{Z}} \wedge S_{+}\right]=\operatorname{hom}(B, \mathbb{Z}\{S\})
$$

this gives a natural transformation

$$
\underline{\pi}_{0} H \underline{\mathbb{Z}} \wedge S_{+} \rightarrow \underline{\mathbb{Z}}\{S\} .
$$

Since both sides take filtered colimits in $S$ to filtered colimits, to check that it is an isomorphism, it suffices to do so when $S$ is finite. In that case we can use the self duality of finite $G$-sets to compute

$$
\left[B_{+}, H \underline{\mathbb{Z}} \wedge S_{+}\right]^{G} \approx\left[B_{+} \wedge S_{+}, H \underline{\mathbb{Z}}\right]^{G},
$$

and then observe that by definition of the constant Mackey functor $\underline{\mathbb{Z}}$, the forgetful map

$$
\left[B_{+} \wedge S_{+}, H \underline{\mathbb{Z}}\right]^{G} \rightarrow\left[B_{+} \wedge S_{+}, H \mathbb{Z}\right]
$$

is an isomorphism with the $G$-invariant part of the target. The claim then follows from the compatibility of equivariant Spanier-Whitehead duality with the restriction functor to non-equivariant spectra.

The properties of permutation Mackey functors listed in the Lemma below follow immediately from the definition. They are used in $\S 4.6 .2$ to establish some of our basic tools for investigating the slice tower. To formulate part ii), note that every $G$-set $B$ receives a functorial map from a free $G$-set, namely $G \times B$, and the group of equivariant automorphisms of $G \times B$ over $B$ is canonically isomorphic to $G$. For instance, one can give $G \times B$ the product of the left action on $G$ and the trivial action on $B$, and take the map $G \times B \rightarrow B$ to be the original action mapping. With this choice the automorphisms $G \times B$ over $B$ are of the form $(g, b) \mapsto\left(g x, x^{-1} b\right)$ with $x \in G$.

Lemma 3.3. Let $\underline{M}$ be a permutation Mackey functor and $B$ finite G-set.

i) If $B^{\prime} \rightarrow B$ is a surjective map of finite $G$-sets, then

$$
\underline{M}(B) \rightarrow \underline{M}\left(B^{\prime}\right) \rightrightarrows \underline{M}\left(B^{\prime} \underset{B}{\times} B^{\prime}\right)
$$

is an equalizer.

ii) Restriction along the action map $G \times B \rightarrow B$ gives an isomorphism

$$
\underline{M}(B) \rightarrow \underline{M}(G \times B)^{G} .
$$

iii) The restriction mapping $\underline{M}(G / H) \rightarrow \underline{M}(G)$ gives an isomorphism

$$
\underline{M}(G / H) \rightarrow \underline{M}(G)^{H}
$$

of $\underline{M}(G / H)$ with the $H$-invariant part of $\underline{M}(G)$.

iv) A map $\underline{M} \rightarrow \underline{M}^{\prime}$ of permutation Mackey functors is an isomorphism if and only if $\underline{M}(G) \rightarrow \underline{M}^{\prime}(G)$ is an isomorphism. 
3.3. Equivariant cellular chains and cochains. The Mackey functor homology and cohomology groups of a $G$-CW spectrum $X$ can be computed from a chain complex analogous to the complex of cellular chains (see, for example [26, §5]). Write $X^{(n)}$ for the $n$-skeleton of $X$ so that

$$
X^{(n)} / X^{(n-1)} \approx X_{n+} \wedge S^{n}
$$

with $X_{n}$ a discrete $G$-set. Set

$$
\begin{aligned}
& C_{n}^{\text {cell }}(X ; \underline{M})=\pi_{n}^{G} H \underline{M} \wedge X^{(n)} / X^{(n-1)}=\pi_{0}^{G} H \underline{M} \wedge X_{n+} \\
& C_{\text {cell }}^{n}(X ; \underline{M})=\left[X^{(n)} / X^{(n-1)}, \Sigma^{n} H \underline{M}\right]^{G}=\left[\Sigma^{\infty} X_{n+}, H \underline{M}\right]^{G} .
\end{aligned}
$$

The map

$$
X^{(n)} / X^{(n-1)} \rightarrow \Sigma X^{(n-1)} / X^{(n-2)}
$$

defines boundary and coboundary maps

$$
\begin{aligned}
C_{n}^{\text {cell }}(X ; \underline{M}) & \rightarrow C_{n-1}^{\text {cell }}(X ; \underline{M}) \\
C_{\text {cell }}^{n-1}(X ; \underline{M}) & \rightarrow C_{\text {cell }}^{n}(X ; \underline{M}) .
\end{aligned}
$$

The equivariant homology and cohomology groups of $X$ with coefficients in $\underline{M}$ are the homology and cohomology groups of these complexes. By writing the $G$-set $X_{n}$ as a coproduct of finite $G$-sets $X_{n}^{\alpha}$ one can express $C_{n}^{\text {cell }}(X ; \underline{M})$ and $C_{\text {cell }}^{n}(X ; \underline{M})$ in terms of the values of the Mackey functor $\underline{M}$ on the $X_{n}^{\alpha}$.

Example 3.4. Write $\rho_{G}$ for the (real) regular representation of $G$ and $\rho_{G}-1$ for the reduced regular representation. The groups

$$
H^{*}\left(S^{\rho_{G}-1} ; \underline{M}\right)
$$

play an important role in equivariant stable homotopy theory. To describe them we need an equivariant cell decomposition of $S^{\rho_{G}-1}$. Since $S^{\rho_{G}-1}$ is the mapping cone of the map

$$
S\left(\rho_{G}-1\right) \rightarrow \mathrm{pt}
$$

from the unit sphere in $\left(\rho_{G}-1\right)$ it suffices to construct an equivariant cell decomposition of $S\left(\rho_{G}-1\right)$. Write $g=|G|$. Think of $\mathbb{R}^{G}$ as the vector space with basis the elements of $G$. The boundary of the standard simplex in this space is equivariantly homeomorphic to $S\left(\rho_{G}-1\right)$. The simplicial decomposition of this simplex is not an equivariant cell decomposition, but the barycentric subdivision is. Thus $S\left(\rho_{G}-1\right)$ is homeomorphic to the geometric realization of the poset of non-empty proper subsets of $G$. This leads to the complex

$$
\underline{M}(G / G) \rightarrow \underline{M}\left(S_{0}\right) \rightarrow \underline{M}\left(S_{1}\right) \rightarrow \cdots \rightarrow \underline{M}\left(S_{g-1}\right)
$$

in which $S_{k}$ is the $G$-set of flags $F_{0} \subset \cdots \subset F_{k} \subset G$ of proper inclusions of subsets of $G$, with $G$ acting by translation. The coboundary map is the alternating sum of the restriction maps derived by omitting one of the sets in a flag.

Corollary 3.6. For any Mackey functor $\underline{M}$, the group

$$
\pi_{\rho_{G}-1}^{G} H \underline{M}=H_{G}^{0}\left(S^{\rho_{G}-1} ; \underline{M}\right)
$$

is given by

$$
\bigcap_{H \subsetneq G} \operatorname{ker}(\underline{M}(G / G) \rightarrow \underline{M}(G / H)) .
$$


Proof: Using the complex (3.5) it suffices to show that the orbit types occurring in $S_{0}$ are precisely the transitive $G$-sets of the form $G / H$ with $H$ a proper subgroup of $G$. The set $S_{0}$ is the set of non-empty proper subsets $S \subset G$. Any proper subgroup $H$ of $G$ occurs as the stabilizer of itself, regarded as a subset of $G$. Since the subsets are proper, the group $G$ does not occur as a stabilizer.

Example 3.7. Let $X$ be the sphere $S^{d-1}$ with the action of $C_{2^{n}}$ given by the antipodal map, and pointed by adding a disjoint base point. The usual cell decomposition into hemispheres is equivariant for the action of $C_{2^{n}}$, and for this cell structure one has $X^{(j)} / X^{(j-1)}=\left(C_{2^{n}} / C_{2^{n-1}}\right)_{+} \wedge S^{j}$. The complex of cellular chains $C_{*}^{\text {cell }}(X ; \underline{M})$ works out to be the complex of length $d$

$$
\underline{M}\left(C_{2^{n}}\right) \longrightarrow \cdots \stackrel{1+\gamma}{\longrightarrow} \underline{M}\left(C_{2^{n}}\right) \stackrel{1-\gamma}{\longrightarrow} \underline{M}\left(C_{2^{n}}\right)
$$

in which $\gamma \in C_{2^{n}}$ is the generator.

Example 3.8. Let $G=C_{2^{n}}$ and $\sigma$ the sign representation of $G$. Suspending the cell decomposition of Example 3.7 gives an equivariant cell decomposition of $S^{d \sigma}$ whose $k$-skeleton is $S^{k \sigma}$ and whose set of $k$-cells is $C_{2+} \times D^{k}$, in which $G$ acts on $C_{2}$ through the unique surjective map $G \rightarrow C_{2}$. The complex of cellular chains $C_{*}^{\text {cell }}\left(S^{d \sigma} ; \underline{M}\right)$ works out to be the complex of length $(d+1)$

$$
\underline{M}\left(C_{2}\right) \longrightarrow \cdots \stackrel{1-\gamma}{\longrightarrow} \underline{M}\left(C_{2}\right) \longrightarrow \underline{M}(\mathrm{pt})
$$

in which $\gamma \in G$ is the preferred generator.

If $\underline{M}$ is the constant Mackey functor $\underline{\mathbb{Z}}$, then $C_{n}^{\text {cell }}(X ; \underline{M})$ is the permutation Mackey functor $\underline{\mathbb{Z}}\left\{X_{n}\right\}$, and associates to a finite $G$-set $B$ the group of equivariant functions

$$
B \rightarrow \mathbb{Z}\left\{X_{n}\right\}=C_{n}^{\text {cell }} X .
$$

In this way the entire Mackey functor chain complex $C_{*}^{\text {cell }}(X ; \underline{\mathbb{Z}})$ is encoded in the ordinary cellular chain complex $C_{*}^{\text {cell }}(X)$ for $i_{0}^{*} X$, equipped with the action of $G$. The equivariant homology group $H_{*}^{G}(X ; \underline{\mathbb{Z}})$ are just the homology groups of the complex

$$
\operatorname{hom}_{G}\left(G / G, C_{*}^{\text {cell }}(X)\right)=C_{*}^{\text {cell }}(X)^{G}
$$

of $G$-invariant cellular chains. Similarly the equivariant cohomology groups $H_{G}^{*}(X ; \mathbb{Z})$ are given by the cohomology groups of the complex

$$
C_{\text {cell }}^{*}(X)^{G}
$$

of equivariant cochains. The equivariant homology and cohomology groups depend only on the equivariant chain homotopy type of these complexes of permutation Mackey functors.

Example 3.9. If $X$ is a $G$-space admitting the structure of a $G$-CW complex, then the cohomology groups $H_{G}^{*}(X ; \underline{\mathbb{Z}})$ are isomorphic to the cohomology groups

$$
H^{*}(X / G ; \mathbb{Z})
$$

of the orbit space. Indeed the equivariant cell decomposition of $X$ induces a cell decomposition of $X / G$ and one has an isomorphism

$$
C_{\text {cell }}^{*}(X)^{G} \approx C_{\text {cell }}^{*}(X / G) .
$$


Example 3.10. Suppose that $V$ is a representation of $G$ of dimension $d$, and consider the equivariant cellular chain complex

$$
C_{d}^{\text {cell }}\left(S^{V} ; \underline{\mathbb{Z}}\right) \rightarrow C_{d-1}^{\text {cell }}\left(S^{V} ; \underline{\mathbb{Z}}\right) \rightarrow \cdots \rightarrow C_{0}^{\text {cell }}\left(S^{V} ; \underline{\mathbb{Z}}\right),
$$

associated to an equivariant cell decomposition of $S^{V}$. The underlying homology groups are those of the sphere $S^{V}$. In particular, the kernel of

$$
C_{d}^{\text {cell }}\left(S^{V} ; \underline{\mathbb{Z}}\right) \rightarrow C_{d-1}^{\text {cell }}\left(S^{V} ; \underline{\mathbb{Z}}\right)
$$

is isomorphic, as a $G$-module, to $H_{d}^{u}\left(S^{V} ; \mathbb{Z}\right)$. If $V$ is orientable then the $G$-action is trivial, and one finds that the restriction map

$$
H_{d}^{G}\left(S^{V} ; \underline{\mathbb{Z}}\right) \rightarrow H_{d}^{u}\left(S^{V} ; \mathbb{Z}\right)
$$

is an isomorphism. A choice of orientation gives an equivariant isomorphism

$$
H_{d}^{u}\left(S^{V} ; \mathbb{Z}\right) \approx \mathbb{Z}
$$

Thus when $V$ is oriented there is a unique isomorphism

$$
H_{d}^{G}\left(S^{V} ; \underline{\mathbb{Z}}\right) \approx \mathbb{Z}
$$

extending the non-equivariant isomorphism given by the orientation.

3.4. Homology and geometric fixed points. In addition to the Mackey functor homotopy groups $\underline{\pi}_{*} X$ there are the $R O(G)$ graded homotopy groups $\pi_{\star}^{G} X$ defined by

$$
\pi_{V}^{G} X=\left[S^{V}, X\right]^{G} \quad V \in R O(G) .
$$

Here $R O(G)$ is the Grothendieck group of real representations of $G$. The use of $\star$ for the wildcard symbol in $\pi_{\star}^{G}$ is taken from Hu-Kriz [35]. The $R O(G)$-graded homotopy groups are also part of a Mackey functor $\underline{\pi}_{\star}(X)$ defined by

$$
\underline{\pi}_{V} X(B)=\left[S^{V} \wedge B_{+}, X\right]^{G} .
$$

As with $\mathbb{Z}$-graded homotopy groups, we'll use the abbreviation

$$
\pi_{V}^{H} X=\left(\underline{\pi}_{V} X\right)(G / H) .
$$

In this section we will make use of $R O(G)$-graded homotopy groups to describe the geometric fixed point spectrum $\Phi^{G} H \underline{\mathbb{Z}}$ when $G=C_{2^{n}}$ (Proposition 3.18 below).

There are a few distinguished elements of $R O(G)$-graded homotopy groups we will need. Let $V$ be a representation of $G$ and $S^{0} \rightarrow S^{V}$ the one point compactification of the inclusion $\{0\} \subset V$.

Definition 3.11. The element

$$
a_{V} \in \pi_{-V}^{G} S^{0}
$$

is the element corresponding under the suspension isomorphism $\pi_{-V}^{G} S^{0} \approx \pi_{0}^{G} S^{V}$ to the map $S^{0} \hookrightarrow S^{V}$ described above.

The element $a_{V}$ is the Euler class of $V$ in $R O(G)$-graded equivariant stable cohomotopy. If $V$ contains a trivial representation then $a_{V}=0$. For two representations $V$ and $W$ one has

$$
a_{V \oplus W}=a_{V} a_{W} \in \pi_{-V-W}^{G} S^{0} .
$$

When $V$ is oriented, Example 3.10 provides a preferred generator of $H_{d}^{G}\left(S^{V} ; \mathbb{Z}\right)$. We give the corresponding $R O(G)$-graded homotopy class name. 
Definition 3.12. Let $V$ be an oriented representation of $G$ of dimension $d$. The element

$$
u_{V} \in \pi_{d-V}^{G} H \underline{\mathbb{Z}} .
$$

is the element corresponding to the preferred generator of $\pi_{d} H \underline{\mathbb{Z}} \wedge S^{V}=H_{d}^{G}\left(S^{V} ; \underline{\mathbb{Z}}\right)$ given by Example 3.10.

If $V$ is trivial then $u_{V}=1$. If $V$ and $W$ are two oriented representations of $G$, and $V \oplus W$ is given the direct sum orientation, then

$$
u_{V \oplus W}=u_{V} u_{W} .
$$

Among other things this implies that the class $u_{V}$ is stable in $V$ in the sense that $u_{V+1}=u_{V}$.

For any $V$, the representation $V \oplus V$ has a canonical orientation, giving

$$
u_{V \oplus V} \in \pi_{2 d-2 V}^{G} H \underline{\mathbb{Z}} \text {. }
$$

When $V$ is oriented this class can be identified, up to sign, with $u_{V}^{2}$.

The elements $a_{V}$ and $u_{V}$ behave well with respect to the norm. The following result is a simple consequence of the fact (2.11) that $N S^{V}=S^{\text {ind } V}$.

Lemma 3.13. Suppose that $V$ is a d-dimensional representation of a subgroup $H \subset G$. Then

$$
\begin{aligned}
N a_{V} & =a_{\text {ind } V} \\
u_{\text {ind } d} \cdot N u_{V} & =u_{\text {ind } V},
\end{aligned}
$$

where ind $V=\operatorname{ind}_{H}^{G} V$ is the induced representation and $d$ is the trivial representation.

Remark 3.14. As is standard in algebra, we will adopt the convention that the operation of mutiplication by an element of a ring on a module is denoted by the element of the ring. We will also use it in closely related contexts. For example, for a $G$-spectrum $X$ we will refer to the to the maps

$$
\begin{aligned}
& a_{V} \wedge 1_{X}: S^{-V} \wedge X \rightarrow X \\
& u_{V} \wedge 1_{X}: S^{d-V} \wedge X \rightarrow H \underline{\mathbb{Z}} \wedge X
\end{aligned}
$$

as multiplication by $a_{V}$ and $u_{V}$ respectively, and, when no confusion is likely, denote them simply by $a_{V}$ and $u_{V}$. Note that $X$ might be a virtual representation sphere. This means that we will not usually distinguish in notation between these maps and their suspensions. Similarly, if $R$ is any equivariant algebra, and $x \in \pi_{V}^{G} S^{0}$ then the product of $x$ with $1 \in \pi_{0}^{G} R$ will be denoted $x \in \pi_{V}^{G} R$. In accordance with this, at various places in this paper the symbol $a_{V}$ might refer to a map $S^{-V} \rightarrow S^{0}$, or its suspension $S^{0} \rightarrow S^{V}$ or the Hurewicz image $S^{0} \rightarrow H \underline{\mathbb{Z}} \wedge S^{V}$ or equivalently an element of $\pi_{0}^{G} H \underline{\mathbb{Z}} \wedge S^{V}$.

Example 3.15. Let $S^{\infty V}$ be the colimit of the spaces $S^{n V}$ under the standard inclusions. Each of these inclusions is "multiplication by $a_{V}$." Smashing with a $G$-spectrum $X$ we find that $S^{\infty V} \wedge X$ is the colimit of the sequence

$$
X \stackrel{a_{V}}{\longrightarrow} S^{V} \wedge X \stackrel{a_{V}}{\longrightarrow} S^{V \oplus V} \wedge X \cdots \stackrel{a_{V}}{\longrightarrow} S^{n V} \wedge X \stackrel{a_{V}}{\longrightarrow} \cdots .
$$

Using the suspension isomorphism to replace $\pi_{\star}^{G} S^{n V} \wedge X$ with $\pi_{\star-n}^{G} X$ the sequence of the $R O(G)$-graded groups becomes

$$
\pi_{\star}^{G} X \stackrel{a_{V}}{\longrightarrow} \pi_{\star-V}^{G} X \cdots \stackrel{a_{V}}{\longrightarrow} \pi_{\star-n V}^{G} X \cdots
$$


from which one gets an isomorphism

$$
\pi_{\star}^{G} S^{\infty V} \wedge X \approx a_{V}^{-1} \pi_{\star}^{G} X .
$$

Under this isomorphism the effect in $R O(G)$-graded homotopy groups induced by the inclusion

$$
S^{n V} \wedge X \rightarrow S^{\infty V} \wedge X
$$

sends $x \in \pi_{\star}^{G} X \approx \pi_{\star}^{G} S^{n V} \wedge X$ to $a_{V}^{-n} x \in a_{V}^{-1} \pi_{\star}^{G} X$.

Example 3.16. Specializing Example 3.8, let $G=C_{2^{n}}$ and $\sigma$ the sign representation. Consider the equivariant homology of $S^{d \sigma}$ with coefficients in the constant Mackey functor $\mathbb{Z}$. The complex of cellular chains works out to be (Example 3.8) the complex of length $(d+1)$

$$
\mathbb{Z} \rightarrow \cdots \mathbb{Z} \stackrel{2}{\rightarrow} \mathbb{Z} \stackrel{0}{\rightarrow} \mathbb{Z} \stackrel{2}{\rightarrow} \mathbb{Z}
$$

Our conventions provide nomenclature for the homology classes. When $d$ is odd the group $H_{d}\left(S^{d \sigma} ; \underline{\mathbb{Z}}\right)$ is zero. When $d$ is even, the representation $d \sigma$ acquires a canonical orientation, the group $H_{d}^{C_{2}}\left(S^{d \sigma} ; \underline{\mathbb{Z}}\right)$ is canonically isomorphic to the integers, and the preferred generator is the class $u_{d \sigma}$ (Remark 3.14). For every even $0 \leq k<d$ the group $H_{k}^{G}\left(S^{d \sigma} ; \underline{\mathbb{Z}}\right)$ is cyclic of order 2 generated by the image of $u_{k \sigma} \in H_{k}^{G}\left(S^{k \sigma} ; \underline{\mathbb{Z}}\right)$ under the map induced by the inclusion $S^{k \sigma} \subset S^{d \sigma}$. As explained in Remark 3.14 this induced map is multiplication by $a_{(d-k) \sigma}$, and so this generator corresponds to the element

$$
a_{(d-k) \sigma} \cdot u_{k \sigma} \in \pi_{k-d \sigma}^{G}(H \underline{\mathbb{Z}})
$$

under the suspension isomorphism

$$
\pi_{k-d \sigma}^{G}(H \underline{\mathbb{Z}}) \approx \pi_{k}^{G}\left(H \underline{\mathbb{Z}} \wedge S^{d \sigma}\right)=H_{k}^{G}\left(S^{d \sigma} ; \underline{\mathbb{Z}}\right) .
$$

Example 3.17. Passing to the limit as $d \rightarrow \infty$ and using the last part of Example 3.15 we find that $a_{(d-k) \sigma} \cdot u_{k \sigma}$ is sent to

$$
a_{d \sigma}^{-1} \cdot a_{(d-k) \sigma} \cdot u_{k \sigma}=a_{k \sigma}^{-1} u_{k \sigma} \in \pi_{k} S^{\infty \sigma} .
$$

Writing $b=a_{2 \sigma}^{-1} u_{2 \sigma}$ we find that the homogeneous component

$$
\pi_{2 n}^{C_{2}} H \underline{\mathbb{Z}} \wedge S^{\infty \sigma} \subset \pi_{\star}^{C_{2}} H \underline{\mathbb{Z}} \wedge S^{\infty \sigma}=a_{2 \sigma}^{-1} \pi^{C_{2}} H \underline{\mathbb{Z}}
$$

is cyclic of order 2 , generated by $b^{n}$.

We now explicitly describe the geometric fixed point spectrum of $H \underline{\mathbb{Z}}$ when $G=C_{2^{n}}$. The computation plays an important role in the proof of the Reduction Theorem.

Proposition 3.18. Let $G=C_{2^{n}}$. For any $G$-spectrum $X$, the $R O(G)$-graded homotopy groups of $\tilde{E} \mathcal{P} \wedge X$ are given by

$$
\pi_{\star}^{G}(\tilde{E} \mathcal{P} \wedge X)=a_{\sigma}^{-1} \pi_{\star}^{G}(X)
$$

The homotopy groups of the commutative algebra $\Phi^{G} H \underline{\mathbb{Z}}$ are given by

$$
\pi_{*}\left(\Phi^{G} H \underline{\mathbb{Z}}\right)=\mathbb{Z} / 2[b],
$$

where $b=u_{2 \sigma} a_{\sigma}^{-2} \in \pi_{2}\left(\Phi^{G} H \underline{\mathbb{Z}}\right)=\pi_{2}^{G}(\tilde{E} \mathcal{P} \wedge H \underline{\mathbb{Z}}) \subset a_{\sigma}^{-1} \pi_{\star}^{G} H \underline{\mathbb{Z}}$. 
Proof: As mentioned in Remark 2.48, the space $\tilde{E} \mathcal{P}$ can be identified with

$$
\lim _{n \rightarrow \infty} S^{n \sigma}
$$

The first assertion therefore follows from Example 3.15. The second assertion follows from Example 3.17 and the fact that the map $a_{\sigma}^{-1} \pi_{\star}^{G} X \rightarrow \pi_{\star}^{G} \tilde{E} \mathcal{P} \wedge X$ is a ring homomorphism when $X$ is an equivariant algebra.

3.5. A gap in homology. We conclude $\S 3$ with some further observations about $S^{\rho_{G}-1}$. Proposition 3.20 below constitutes the computational part of the Gap Theorem, and contains the Cell Lemma as a special case.

Example 3.19. Suppose that $G$ is not the trivial group. In $\S 4.6 .2$ we will encounter the group

$$
\pi_{\rho_{G}-2}^{G} H \underline{\mathbb{Z}} \approx H_{G}^{1}\left(S^{\rho_{G}-1} ; \underline{\mathbb{Z}}\right)
$$

which, by Example 3.9, is isomorphic to

$$
H^{1}\left(S^{\rho_{G}-1} / G ; \mathbb{Z}\right) .
$$

The $G$-space $S^{\rho_{G}-1}$ is the unreduced suspension of the unit sphere $S\left(\rho_{G}-1\right)$, and so the orbit space is also a suspension. If $|G|>2$ then $S\left(\rho_{G}-1\right)$ is connected, hence so is the orbit space. If $G=C_{2}$, then $S\left(\rho_{G}-1\right) \approx G$ and the orbit space is still connected. In all cases then, the unreduced suspension $S^{\rho_{G}-1} / G$ is simply connected. Thus

$$
\pi_{\rho_{G}-2}^{G} H \underline{\mathbb{Z}} \approx H_{G}^{2}\left(S^{\rho_{G}} ; \underline{\mathbb{Z}}\right) \approx H_{G}^{1}\left(S^{\rho_{G}-1} ; \underline{\mathbb{Z}}\right)=0 .
$$

In fact, the same argument shows that for $n>0$ the orbit space $S^{n\left(\rho_{G}-1\right)} / G$ is simply connected, and hence

$$
H_{G}^{0}\left(S^{n\left(\rho_{G}-1\right)} ; \underline{\mathbb{Z}}\right)=H_{G}^{1}\left(S^{n\left(\rho_{G}-1\right)} ; \underline{\mathbb{Z}}\right)=0
$$

or, equivalently

$$
\pi_{n\left(\rho_{G}-1\right)}^{G} H \underline{\mathbb{Z}}=\pi_{n\left(\rho_{G}-1\right)-1}^{G} H \underline{\mathbb{Z}}=0 .
$$

Building on this, we have

Proposition 3.20. Let $G$ be any non-trivial finite group and $n \geq 0$ an integer. Except in case $G=C_{3}, i=3, n=1$ the groups

$$
H_{G}^{i}\left(S^{n \rho_{G}} ; \underline{\mathbb{Z}}\right)
$$

are zero for $0<i<4$. In the exceptional case one has

$$
H_{G}^{3}\left(S^{\rho_{C_{3}}} ; \underline{\mathbb{Z}}\right)=\mathbb{Z} \text {. }
$$

Proof: Since

$$
H_{G}^{i}\left(S^{n \rho_{G}} ; \underline{\mathbb{Z}}\right) \approx H_{G}^{i-n}\left(S^{n\left(\rho_{G}-1\right)} ; \underline{\mathbb{Z}}\right),
$$

connectivity and Example 3.19 show that $H_{G}^{i}\left(S^{n \rho_{G}} ; \underline{\mathbb{Z}}\right)=0$ for $i \leq n+1$. This takes care of the cases in which $n+1 \geq 3$, leaving only $n=1$, and in that case only the group

which is isomorphic to

$$
H_{G}^{2}\left(S^{\rho_{G}-1} ; \underline{\mathbb{Z}}\right)
$$

$$
H^{2}\left(S^{\rho_{G}-1} / G ; \mathbb{Z}\right) .
$$


Since the orbit space $S^{\rho_{G}-1} / G$ is simply connected, the universal coefficient theorem gives an inclusion

$$
H^{2}\left(S^{\rho_{G}-1} / G ; \mathbb{Z}\right) \rightarrow H^{2}\left(S^{\rho_{G}-1} / G ; \mathbb{Q}\right) .
$$

It therefore suffices to show that

$$
H^{2}\left(S^{\rho_{G}-1} / G ; \mathbb{Q}\right)=0 .
$$

But since $G$ is finite, this group is just the $G$-invariant part of

$$
H^{2}\left(S^{\rho_{G}-1} ; \mathbb{Q}\right)
$$

which is zero since $G$ does not have order 3 . When $G$ does have order 3 the group is $\mathbb{Q}$. The claim follows since the homology groups are finitely generated.

\section{THE SLICE FILTRATION}

The slice filtration is an equivariant analogue of the Postnikov tower, to which it reduces in the case of the trivial group. In this section we introduce the slice filtration and establish some of its basic properties. We work for the most part with a general finite group $G$, though our application to the Kervaire invariant problem involves only the case $G=C_{2^{n}}$. While the situation for general $G$ exhibits many remarkable properties, the reader should regard as exploratory the apparatus of definitions at this level of generality.

From now until the end of $\S 11$ our focus will be on homotopy theory. Though it will not appear in the notation, all spectra should be replaced by cofibrant or fibrant approximations where appropriate.

\subsection{Slice cells.}

4.1.1. Slice cells and their dimension. For a subgroup $K \subset G$ let $\rho_{K}$ denote its regular representation, and write

$$
\widehat{S}(m, K)=G_{+} \bigwedge_{K} S^{m \rho_{K}} \quad m \in \mathbb{Z} .
$$

Definition 4.1. The set of slice cells is

$$
\left\{\widehat{S}(m, K), \Sigma^{-1} \widehat{S}(m, K) \mid m \in \mathbb{Z}, K \subset G\right\} .
$$

This brings two notions of "cell" into the story: the slice cells and the cells of the form $G / H_{+} \wedge D^{m}$, used to manufacture $G$-CW spectra. We'll refer to the traditional equivariant cells as " $G$-cells" in order to distinguish them from the slice cells.

Definition 4.2. A slice cell is regular if it is of the form $\widehat{S}(m, K)$.

Definition 4.3. A slice cell is induced if it is of the form

$$
G_{+} \bigwedge_{H} \widehat{S}
$$

where $\widehat{S}$ is a slice cell for $H$ and $H \subset G$ is a proper subgroup. It is free if $H$ is the trivial group. A slice cell is isotropic if it is not free. 
Since

$$
\begin{aligned}
& {\left[G_{+} \bigwedge_{H} S, X\right]^{G} \approx\left[S, i_{H}^{*} X\right]^{H} \quad \text { and }} \\
& {\left[X, G_{+} \bigwedge_{H} S\right]^{G} \approx\left[i_{H}^{*} X, S\right]^{H}}
\end{aligned}
$$

induction on $|G|$ usually reduces claims about cells to the case of those which are not induced. The slice cells which are not induced are those of the form $S^{m \rho_{G}}$ and $S^{m \rho_{G}-1}$.

Definition 4.4. The dimension of a slice cell is defined by

$$
\begin{aligned}
\operatorname{dim} \widehat{S}(m, K) & =m|K| \\
\operatorname{dim} \Sigma^{-1} \widehat{S}(m, K) & =m|K|-1 .
\end{aligned}
$$

In other words the dimension of a slice cell is that of its underlying spheres.

Remark 4.5. Not every suspension of a slice cell is a slice cell. Typically, the spectrum $\Sigma^{-2} \widehat{S}(m, K)$ will not be a slice cell, and will not exhibit the properties of a slice cell of dimension $\operatorname{dim} \widehat{S}(m, K)-2$.

The following is immediate from the definition.

Proposition 4.6. Let $H \subset G$ be a subgroup. If $\widehat{S}$ is a $G$-slice cell of dimension $d$, then $i_{H}^{*} \widehat{S}$ is a wedge of $H$-slice cells of dimension d. If $\widehat{S}$ is an $H$-slice cell of dimension d then $G_{+}{ }_{H} \widehat{S}$ is a G-slice cell of dimension $d$.

The regular slice cells behave well under the norm.

Proposition 4.7. Let $H \subset G$ be a subgroup. If $\widehat{W}$ is a wedge of regular $H$-slice cells, then $N_{H}^{G} \widehat{W}$ is a wedge of regular G-slice cells.

Proof: The wedges of regular $H$-slice cells are exactly the indexed wedges (in the sense of $\S 2.3 .2)$ of spectra of the form $S^{m \rho_{K}}$ for $K \subset H$, and $m \in \mathbb{Z}$. Since regular representations induce to regular representations, the identity (2.11) and the distribution formula (Proposition A.37) show that the norm of such an indexed wedge is an indexed wedge of $S^{m \rho_{K}}$ with $K \subset G$ and $m \in \mathbb{Z}$. The claim follows.

4.1.2. Slice positive and slice null spectra. Underlying the theory of the Postnikov tower is the notion of "connectivity" and the class of $(n-1)$-connected spectra. In this section we describe the slice analogues of these ideas. There is a simple relationship between "connectivity" and "slice-positivity" which we will describe in detail in $\$ 4.4$.

Definition 4.8. A $G$-spectrum $Y$ is slice $n$-null, written

$$
Y<n \quad \text { or } \quad Y \leq n-1
$$

if for every slice cell $\widehat{S}$ with $\operatorname{dim} \widehat{S} \geq n$ the $G$-space

$$
\underline{\mathcal{S}}_{G}(\widehat{S}, Y)
$$

is equivariantly contractible. A $G$-spectrum $X$ is slice $n$-positive, written

$$
X>n \quad \text { or } \quad X \geq n+1
$$


if

$$
\underline{\mathcal{S}}_{G}(X, Y)
$$

is equivariantly contractible for every $Y$ with $Y \leq n$.

We will use the terms slice-positive and slice-null instead of "slice 0-positive" and "slice 0-null." The full subcategory of $\mathcal{S}^{G}$ consisting of $X$ with $X>n$ will be denoted $\mathcal{S}_{>n}^{G}$ or $\mathcal{S}_{\geq n+1}^{G}$. Similarly, the full subcategory of $\mathcal{S}^{G}$ consisting of $X$ with $X<n$ will be denoted $\mathcal{S}_{<n}^{G}$ or $\mathcal{S}_{\leq n-1}^{G}$.

Remark 4.9. The category $\mathcal{S}_{>n}^{G}$ is the smallest full subcategory of $\mathcal{S}^{G}$ containing the slice cells $\widehat{S}$ with $\operatorname{dim} \widehat{S}>n$ and possessing the following properties:

i) If $X$ is weakly equivalent to an object of $\mathcal{S}_{>n}^{G}$, then $X$ is in $\mathcal{S}_{>n}^{G}$.

ii) Arbitrary wedges of objects of $\mathcal{S}_{>n}^{G}$ are in $\mathcal{S}_{>n}^{G}$.

iii) If $X \rightarrow Y \rightarrow Z$ is a cofibration sequence and $X$ and $Y$ are in $\mathcal{S}_{>n}^{G}$ then so is $Z$.

iv) If $X \rightarrow Y \rightarrow Z$ is a cofibration sequence and $X$ and $Z$ are in $\mathcal{S}_{>n}^{G}$ then so is $Y$.

More briefly, these properties are that $\mathcal{S}_{>n}^{G}$ is closed under weak equivalences, homotopy colimits (properties ii) and iii)), and extensions.

Remark 4.10. The fiber of a map of slice $n$-positive spectra is not assumed to be slice $n$-positive, and need not be. For example, the fiber of $* \rightarrow S^{\rho_{G}}$ is $S^{\rho_{G}-1}$ which is not slice $(|G|-1)$-positive, even though both $*$ and $S^{\rho_{G}}$ are.

For $n=0,-1$, the notions of slice $n$-null and slice $n$-positive are familiar.

Proposition 4.11. For a $G$-spectrum $X$ the following hold

i) $X \geq 0 \Longleftrightarrow X$ is $(-1)$-connected, i.e. $\underline{\pi}_{k} X=0$ for $k<0$;

ii) $X \geq-1 \Longleftrightarrow X$ is (-2)-connected, i.e. $\underline{\pi}_{k} X=0$ for $k<-1$;

iii) $X<0 \Longleftrightarrow X$ is 0 -coconnected, i.e. $\underline{\pi}_{k} X=0$ for $k \geq 0$;

iv) $X<-1 \Longleftrightarrow X$ is $(-1)$-coconnected, i.e. $\underline{\pi}_{k} X=0$ for $k \geq-1$;

Proof: These are all straightforward consequences of the fact that $S^{0}$ is a slice cell of dimension 0 , and $S^{-1}$ is a slice cell of dimension $(-1)$.

Remark 4.12. It is not the case that if $Y>0$ then $\pi_{0} Y=0$. In Proposition 4.15 we will see that the fiber $F$ of $S^{0} \rightarrow H \underline{\mathbb{Z}}$ has the property that $F>0$. On the other hand $\underline{\pi}_{0} F$ is the augmentation ideal of the Burnside ring. Proposition 4.48 below gives a characterization of slice-positive spectra.

The classes of slice $n$-null and slice $n$-positive spectra are preserved under change of group.

Proposition 4.13. Suppose $H \subset G$, that $X$ is a $G$-spectrum and $Y$ is an $H$ spectrum. The following implications hold

$$
\begin{aligned}
& X>n \Longrightarrow i_{H}^{*} X>n \\
& X<n \Longrightarrow i_{H}^{*} X<n \\
& Y>n \Longrightarrow G_{+}{ }_{H} Y>n \\
& Y<n \Longrightarrow G_{+}{ }_{H} Y<n .
\end{aligned}
$$


Proof: The second and third implications are straightforward consequences of Proposition 4.6. The fourth implication follows from the Wirthmüller isomorphism and Proposition 4.6, and the first implication is an immediate consequence of the fourth.

We end this section with a mild simplification of the condition that a spectrum be slice $n$-null.

Lemma 4.14. For a $G$-spectrum $X$, the following are equivalent

i) $X<n$;

ii) For all slice cells $\widehat{S}$ with $\operatorname{dim} \widehat{S} \geq n,[\widehat{S}, X]^{G}=0$.

Proof: The first condition trivially implies the second. We prove that the second implies the first by induction on $|G|$. By the induction hypothesis we may assume that the $G$-space

$$
\underline{\mathcal{S}}_{G}(\widehat{S}, X)
$$

is contractible for all induced slice cells $\widehat{S}$ with $\operatorname{dim} \widehat{S} \geq n$, and that for all slice cells $\widehat{S}$ with $\operatorname{dim} \widehat{S} \geq n$, and all proper $H \subset G$, the space

$$
\underline{S}_{G}(\widehat{S}, X)^{H}
$$

is contractible. We therefore also know that the $G$-space

$$
\underline{S}_{G}(T \wedge \widehat{S}, X)
$$

is contractible, for all slice cells $\widehat{S}$ with $\operatorname{dim} \widehat{S} \geq n$ and all (-1)-connected $G$-CW spectra $T$ built entirely from induced $G$-cells. We must show that for each $t \geq 0$, the groups

$$
\begin{gathered}
{\left[S^{t} \wedge S^{m \rho_{G}-1}, X\right]^{G} \quad m|G|-1 \geq n} \\
{\left[S^{t} \wedge S^{m \rho_{G}}, X\right]^{G} \quad m|G| \geq n}
\end{gathered}
$$

are zero. They are zero by assumption when $t=0$. For $t>0$, the first case is a special case of the second, since $S^{1} \wedge S^{m \rho_{G}-1}$ is a slice cell of dimension $m|G|$. Let $T$ be the homotopy fiber of the map

$$
S^{t} \subset S^{t \rho_{G}},
$$

and consider the exact sequence

$$
\left[S^{t \rho_{G}} \wedge S^{m \rho_{G}}, X\right]^{G} \rightarrow\left[S^{t} \wedge S^{m \rho_{G}}, X\right]^{G} \rightarrow\left[T \wedge S^{m \rho_{G}}, X\right]^{G} .
$$

The leftmost group is zero since $S^{t \rho_{G}} \wedge S^{m \rho_{G}}$ is a slice cell of dimension $(t+$ $m)|G| \geq n$. The rightmost group is zero by the induction hypothesis since $T$ is $(-1)$-connected and built entirely from induced $G$-cells. It follows from exactness that the middle group is zero. 
4.2. The slice tower. Let $P^{n} X$ be the Bousfield localization, or Dror Farjoun nullification $([19,30])$ of $X$ with respect to the class $\mathcal{S}_{>n}^{G}$, and $P_{n+1} X$ the homotopy fiber of $X \rightarrow P^{n} X$. Thus, by definition, there is a functorial fibration sequence

$$
P_{n+1} X \rightarrow X \rightarrow P^{n} X
$$

The functor $P^{n} X$ can be constructed as the colimit of a sequence of functors

$$
W_{0} X \rightarrow W_{1} X \rightarrow \cdots \text {. }
$$

The $W_{i} X$ are defined inductively starting with $W_{0} X=X$, and taking $W_{k} X$ to be the cofiber of

$$
\bigvee_{I} \Sigma^{t} \widehat{S} \rightarrow W_{k-1} X
$$

in which the indexing set $I$ is the set of maps $\Sigma^{t} \widehat{S} \rightarrow W_{k-1} X$ with $\widehat{S}>n$ a slice cell and $t \geq 0$. By Lemma 4.14 the functors $P^{n}$ can also be formed using the analogous construction using only slice cells themselves, and not their suspensions.

Proposition 4.15. A spectrum $X$ is slice $n$-positive if an only if it admits (up to weak equivalence) a filtration

$$
X_{0} \subset X_{1} \subset \cdots
$$

whose associated graded spectrum $\bigvee X_{k} / X_{k-1}$ is a wedge of slice cells of dimension greater than $n$. For any spectrum $X, P_{n+1} X$ is slice n-positive.

Proof: This follows easily from the construction of $P^{n} X$ described above.

The map $P_{n+1} X \rightarrow X$ is characterized up to a contractible space of choices by the properties

i) for all $X, P_{n+1} X \in \mathcal{S}_{>n}^{G}$;

ii) for all $A \in \mathcal{S}_{>n}^{G}$ and all $X$, the map $\underline{\mathfrak{S}}_{G}\left(A, P_{n+1} X\right) \rightarrow \underline{\mathfrak{S}}_{G}(A, X)$ is a weak equivalence of $G$-spaces.

In other words, $P_{n+1} X \rightarrow X$ is the "universal map" from an object of $\mathcal{S}_{>n}^{G}$ to $X$. Similarly $X \rightarrow P^{n} X$ is the universal map from $X$ to a slice $(n+1)$-null $G$-spectrum $Z$. More specifically

iii) the spectrum $P^{n} X$ is slice $(n+1)$-null;

iv) for any slice $(n+1)$-null $Z$, the map

$$
\underline{\mathfrak{S}}_{G}\left(P^{n} X, Z\right) \rightarrow \underline{\mathfrak{S}}_{G}(X, Z)
$$

is a weak equivalence.

These conditions lead to a useful recognition principle.

Lemma 4.16. Suppose $X$ is a $G$-spectrum and that

$$
\tilde{P}_{n+1} \rightarrow X \rightarrow \tilde{P}^{n}
$$

is a fibration sequence with the property that $\tilde{P}^{n} \leq n$ and $\tilde{P}_{n+1}>n$. Then the canonical maps $\tilde{P}_{n+1} \rightarrow P_{n+1} X$ and $P^{n} X \rightarrow \tilde{P}^{n}$ are weak equivalences.

Proof: We show that the map $X \rightarrow \tilde{P}^{n}$ satisfies the universal property of $P^{n} X$. Suppose that $Z \leq n$, and consider the fibration sequence of $G$-spaces

$$
\underline{\mathfrak{S}}_{G}\left(\tilde{P}^{n}, Z\right) \rightarrow \underline{\mathfrak{S}}_{G}(X, Z) \rightarrow \underline{\mathfrak{S}}_{G}\left(\tilde{P}_{n+1}, Z\right)
$$


The rightmost space is contractible since $\tilde{P}_{n+1}>n$, so the map $\underline{\mathcal{S}}_{G}\left(\tilde{P}^{n}, Z\right) \rightarrow$ $\underline{\mathcal{S}}_{G}(X, Z)$ is a weak equivalence.

The following consequence of Lemma 4.16 is used in the proof of the Reduction Theorem.

Corollary 4.17. Suppose that $X \rightarrow Y \rightarrow Z$ is a cofibration sequence, and that the mapping cone of $P^{n} X \rightarrow P^{n} Y$ is slice $(n+1)$-null. Then both

$$
P^{n} X \rightarrow P^{n} Y \rightarrow P^{n} Z
$$

and

$$
P_{n+1} X \rightarrow P_{n+1} Y \rightarrow P_{n+1} Z
$$

are cofibration sequences.

Proof: Consider the diagram

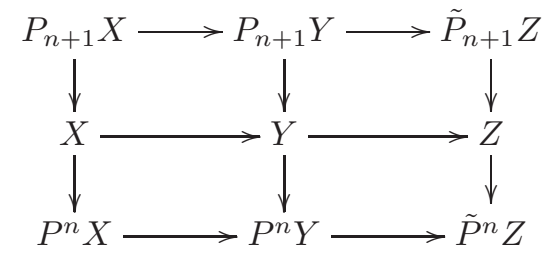

in which the rows and columns are cofibration sequences. By construction, $\tilde{P}_{n+1} Z$ is slice $n$-positive (Remark 4.9). If $\tilde{P}^{n} Z \leq n$ then the right column satisfies the condition of 4.16 , and the result follows.

Since $\mathcal{S}_{>n}^{G} \subset \mathcal{S}_{>n-1}^{G}$, there is a natural transformation

$$
P^{n} X \rightarrow P^{n-1} X
$$

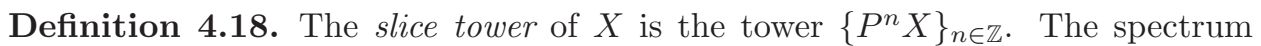
$P^{n} X$ is the $n^{\text {th }}$ slice section of $X$.

When considering more than one group, we will write $P^{n} X=P_{G}^{n} X$ and $P_{n} X=$ $P_{n}^{G} X$.

Let $P_{n}^{n} X$ be the fiber of the map

$$
P^{n} X \rightarrow P^{n-1} X
$$

Definition 4.19. The $n$-slice of a spectrum $X$ is $P_{n}^{n} X$. A spectrum $X$ is an $n$-slice if $X=P_{n}^{n} X$.

The spectrum $P_{n+1} X$ is analogous to the $n$-connected cover of $X$, and for two values of $n$ they coincide. The following is a straightforward consequence of Proposition 4.11 .

Proposition 4.20. For any spectrum $X, P_{0} X$ is the $(-1)$-connected cover of $X$ and $P_{-1} X$ is the (-2)-connected cover of $X$. The $(-1)$-slice of $X$ is given by

$$
P_{-1}^{-1} X=\Sigma^{-1} H_{-1} X .
$$

The formation of slice sections and therefore of the slices themselves behave well with respect to change of group. 
Proposition 4.21. The functor $P^{n}$ commutes with both restriction to a subgroup and left induction. More precisely, given $H \subset G$ there are natural weak equivalences

$$
i_{H}^{*}\left(P_{G}^{n} X\right) \rightarrow P_{H}^{n}\left(i_{H}^{*} X\right)
$$

and

$$
G_{+} \underset{H}{\wedge}\left(P_{H}^{n} X\right) \rightarrow P_{G}^{n}\left(G_{+} \underset{H}{\wedge} X\right)
$$

Proof: This is an easy consequence of Lemma 4.16 and Proposition 4.13.

Remark 4.22. When $G$ is the trivial group the slice cells are just ordinary cells and the slice tower becomes the Postnikov tower. It therefore follows from Proposition 4.21 that the tower of non-equivariant spectra underlying the slice tower is the Postnikov tower.

4.3. Multiplicative properties of the slice tower. The slice filtration does not quite have the multiplicative properties one might expect. In this section we collect a few results describing how things work. One important result is Corollary 4.32 asserting that the slice sections of a $(-1)$-connected commutative or associative algebra are (-1)-connected commutative or associative algebras. We'll show in $\S 4.7$ the slice filtration is multiplicative for the special class of "pure" spectra, defined in $\S 4.6 .2$.

Lemma 4.23. Smashing with $S^{m \rho_{G}}$ gives a bijection of the set of slice cells $\widehat{S}$ with $\operatorname{dim} \widehat{S}=k$ and those with $\operatorname{dim} \widehat{S}=k+m|G|$.

Proof: Since the restriction of $\rho_{G}$ to $K \subset G$ is $|G / K| \rho_{K}$ there is an identity

$$
S^{\rho_{G}} \wedge\left(G_{+}{ }_{K}^{\wedge} S^{m \rho_{K}}\right) \approx G_{+} \bigwedge_{K}\left(S^{\rho_{G}} \wedge S^{m \rho_{K}}\right) \approx G_{+}{ }_{K}^{\wedge} S^{(|G / K|+m) \rho_{K}} .
$$

The result follows easily from this.

Corollary 4.24. Smashing with $S^{m \rho_{G}}$ gives an equivalence

$$
\mathcal{S}_{\geq n}^{G} \rightarrow \mathcal{S}_{\geq n+m|G|}^{G} .
$$

Corollary 4.25. The natural maps

$$
\begin{gathered}
S^{m \rho_{G}} \wedge P_{k+1} X \rightarrow P_{k+m|G|+1}\left(S^{m \rho_{G}} \wedge X\right) \\
S^{m \rho_{G}} \wedge P^{k} X \rightarrow P^{k+m|G|}\left(S^{m \rho_{G}} \wedge X\right)
\end{gathered}
$$

are weak equivalences.

Proposition 4.26. If $X \geq n, Y \geq m$, and $n$ is divisible by $|G|$ then $X \wedge Y \geq n+m$.

Proof: By smashing $X$ with $S^{(-n /|G|) \rho_{G}}$ and using Corollary 4.25 we may assume $n=0$. Suppose that $Z<m$. Since $Y \geq m$, the zero space of function spectrum $Z^{Y}$ is contractible, and so $Z^{Y}$ is 0 -coconnected. Since $X$ is $(-1)$-connected (Proposition 4.11)

$$
\underline{\mathcal{S}}_{G}(X \wedge Y, Z) \approx \underline{\mathfrak{S}}_{G}\left(X, Z^{Y}\right)
$$

is contractible and so $X \wedge Y \geq m$. 
Definition 4.27. A map $X \rightarrow Y$ is a $P^{n}$-equivalence if $P^{n} X \rightarrow P^{n} Y$ is an equivalence. Equivalently, $X \rightarrow Y$ is a $P^{n}$-equivalence if for every $Z<n$, the map

$$
\underline{\mathfrak{S}}_{G}(Y, Z) \rightarrow \underline{\mathfrak{S}}_{G}(X, Z)
$$

is a weak equivalence.

Lemma 4.28. If the homotopy fiber $F$ of $f: X \rightarrow Y$ is in $\mathcal{S}_{>n}^{G}$, then $f$ is a a $P^{n}$ equivalence.

Proof: Immediate from the fibration sequence

$$
\underline{\mathcal{S}}_{G}(Y, Z) \rightarrow \underline{\mathfrak{S}}_{G}(X, Z) \rightarrow \underline{\mathfrak{S}}_{G}(F, Z) .
$$

Remark 4.29. The converse of the above result is not true. For instance, $* \rightarrow S^{0}$ is a $P^{-1}$-equivalence, but the fiber $S^{-1}$ is not in $\mathcal{S}_{>-1}^{G}$.

Lemma 4.30. i) If $Y \rightarrow Z$ is a $P^{n}$-equivalence and $X \geq 0$, then $X \wedge Y \rightarrow X \wedge Z$ is a $P^{n}$-equivalence;

ii) For $X_{1}, \ldots, X_{k} \in \mathcal{S}_{\geq 0}^{G}$, the map

$$
X_{1} \wedge \cdots \wedge X_{k} \rightarrow P^{n} X_{1} \wedge \cdots \wedge P^{n} X_{k}
$$

is a $P^{n}$-equivalence.

Proof: Since $P_{n+1} X$ and $P_{n+1} Y$ are both slice $n$-positive the vertical map in the square below are $P^{n}$-equivalences by Lemmas 4.28 and 4.26

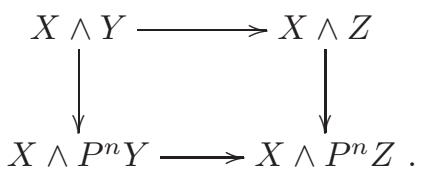

The bottom row is a weak equivalence by assumption. It follows that the top row is a $P^{n}$-equivalence. The second assertion is proved by induction on $k$, the case $k=1$ being trivial. For the induction step consider

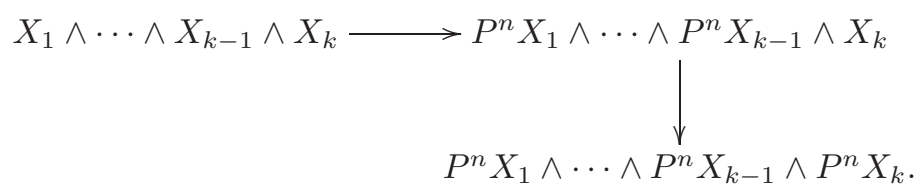

The first map is a $P^{n}$-equivalence by the induction hypothesis and part i). The second map is a $P^{n}$-equivalence by part i).

Remark 4.31. Lemma 4.30 can be described as asserting that the functor

$$
P^{n}:\{(-1) \text {-connected spectra }\} \rightarrow\left\{\mathcal{S}_{>n}^{G} \text {-null spectra }\right\}
$$

is weakly monoidal.

Corollary 4.32. Let $R$ be a (-1)-connected $G$-spectrum. If $R$ is a homotopy commutative or homotopy associative algebra, then so is $P^{n} R$ for all $n$. 
The following additional results are proved in §B.9. The first two are Propositions B.171, B.177, and the third is easily deduced from Proposition B.179.

Proposition 4.33. Suppose that $n \geq 0$ is an integer. If $A$ is a slice $(n-1)$-positive $H$-spectrum then $N_{H}^{G} A$ is a slice $(n-1)$-positive $G$-spectrum.

Proposition 4.34. Suppose that $n \geq 0$ is an integer. If $A$ is a slice $(n-1)$ positive $G$-spectrum then for every $m>0$, the symmetric smash power $\mathrm{Sym}^{m} A$ is slice $(n-1)$-positive.

Proposition 4.35. Suppose that $n \geq 0$ is an integer. If $R$ is a $(-1)$-connected equivariant commutative ring, then the slice section $P^{n} R$ can be given a the structure of an equivariant commutative ring in such a way that $R \rightarrow P^{n} R$ is a commutative ring homomorphism. Moreover this commutative ring structure is unique.

4.4. The slice spectral sequence. The slice spectral sequence is the homotopy spectral of the slice tower. The main point of this section is to establish strong convergence of the slice spectral sequence, and to show that for any $X$ the $E_{2}$-term is distributed in the gray region of Figure 1. We begin with some results relating the slice sections to Postnikov sections.

4.4.1. Connectivity and the slice filtration. Our convergence result for the slice spectral sequence depends on knowing how slice cells are constructed from $G$-cells. We will say that a space or spectrum $X$ decomposes into the elements of a collection of spectra $\left\{T_{\alpha}\right\}$ if $X$ is weakly equivalent to a spectrum $\tilde{X}$ admitting an increasing filtration

$$
X_{0} \subset X_{1} \subset \cdots
$$

with the property that $X_{n} / X_{n-1}$ is weakly equivalent to a wedge of $T_{\alpha}$.

Remark 4.36. A $G$-spectrum $X$ decomposes into a collection of spectra $\left\{G / H_{+} \wedge\right.$ $S^{m}$, with $H$ and $m$ ranging through some indexing list, if and only if $X$ is weakly equivalent a $G$-CW spectrum with $G$-cells of the form $G / H_{+} \wedge D^{m}$, with $H$ and $m$ ranging through the same list.

Remark 4.37. To say that $X$ decomposes into the elements of a collection of compact objects $\left\{T_{\alpha}\right\}$ means that $X$ is in the smallest subcategory of $\underline{S}_{G}$ closed under weak equivalences, arbitrary wedges, and the formation of mapping cones and extensions (i.e., the properties listed in Remark 4.9).

Lemma 4.38. Let $\widehat{S}$ be a slice cell. If $\operatorname{dim} \widehat{S}=n \geq 0$, then $\widehat{S}$ decomposes into the spectra $G / H_{+} \wedge S^{k}$ with $\lfloor n /|G|\rfloor \leq k \leq n$. If $\operatorname{dim} \widehat{S}=n<0$ then $\widehat{S}$ decomposes into $G / H_{+} \wedge S^{k}$ with $n \leq k \leq\lfloor n /|G|\rfloor$.

Proof: The cell structure of $S^{\rho_{G}-1}$ described in Example 3.4 has $G$-cells ranging in dimension from 0 to $|G|-1$, and suspends to a cell decomposition of $S^{\rho_{G}}$ with $G$-cells whose dimension ranges from 1 to $|G|$. The cases $\widehat{S}=S^{m \rho_{G}}$ and $\widehat{S}=S^{m \rho_{G}-1}$ with $m \geq 0$ are handled by smashing these together and passing to suspension spectra. For $m<0$, Spanier-Whitehead duality gives an equivariant cell decomposition of $S^{m \rho_{G}}$ into cells whose dimensions range from $m|G|$ to $m$ and of $\Sigma^{-1} S^{m \rho_{G}}$ into cells whose dimensions range from $n=m|G|-1$ to $m-1=\lfloor n /|G|\rfloor$. Finally, the case in which $\widehat{S}$ is induced from a subgroup $K \subset G$ is proved by left inducing its $K$-equivariant cell decomposition. 
Corollary 4.39. Let $Y \in \mathcal{S}_{\geq_{n}}^{G}$. If $n \geq 0$, then $Y$ can be decomposed into the spectra $G / H_{+} \wedge S^{m}$ with $m \geq\lfloor n /|G|\rfloor$. If $n \leq 0$ then $Y$ can be decomposed into $G / H_{+} \wedge S^{m}$ with $m \geq n$.

Proof: The class of $G$-spectra $Y$ which can be decomposed into $G / H_{+} \wedge S^{m}$ with $m \geq\lfloor n /|G|\rfloor$ is closed under weak equivalences, homotopy colimits, and extensions. By Lemma 4.38 it contains the slice cells $\widehat{S}$ with $\operatorname{dim} \widehat{S} \geq n$. It therefore contains all $Y \in \mathcal{S}_{\geq n}^{G}$ by Remark 4.9. A similar argument handles the case $n<0$.

Proposition 4.40. Write $g=|G|$.

i) If $n \geq 0$ and $k>n$ then $(G / H)_{+} \wedge S^{k}>n$.

ii) If $m \leq-1$ and $k \geq m$ then $(G / H)_{+} \wedge S^{k} \geq(m+1) g-1$.

iii) If $Y \geq n$ with $n \geq 0$, then $\underline{\pi}_{i} Y=0$ for $i<\lfloor n / g\rfloor$.

iv) If $Y \geq n$ with $n \leq 0$, then $\underline{\pi}_{i} Y=0$ for $i<n$.

Proof: We start with the first assertion. We will prove the claim by induction on $|G|$, the case of the trivial group being obvious. Using Proposition 4.13 we may assume by induction that $(G / H)_{+} \wedge S^{k}>n$ when $n \geq 0$ and $H \subset G$ is a proper subgroup. This implies that if $T$ is an equivariant $\mathrm{CW}$-spectrum built from $G$-cells of the form $(G / H)_{+} \wedge S^{k}$ with $k>n$ and $H \subset G$ a proper subgroup, then $T>n$. The homotopy fiber of the natural inclusion

$$
S^{k} \rightarrow S^{k \rho_{G}}
$$

can be identified with the suspension spectrum of $S\left(k \rho_{G}-k\right)_{+} \wedge S^{k}$, and so is such a $T$. Since $S^{k \rho_{G}} \geq k|G| \geq k g>n$ the fibration sequence

$$
T \rightarrow S^{k} \rightarrow S^{k \rho_{G}}
$$

exhibits $S^{k}$ as an extension of two slice $n$-positive spectra, making it slice $n$-positive. The second assertion is trivial for $k \geq 0$ since in that case $G / H_{+} \wedge S^{k} \geq 0$ and $(m+1) g-1 \leq-1$. The case $k \leq-1$ is handled by writing

$$
(G / H)_{+} \wedge S^{k}=\Sigma^{-1}(G / H)_{+} \wedge S^{(k+1) \rho_{G}} \wedge S^{-(k+1)\left(\rho_{G}-1\right)} .
$$

Since $-(k+1) \geq 0$, the spectrum $S^{-(k+1)\left(\rho_{G}-1\right)}$ is a suspension spectrum and so

$$
(G / H)_{+} \wedge S^{k} \geq(k+1) g-1 \geq(m+1) g-1 .
$$

The third and fourth assertions are immediate from Corollary 4.39.

Remark 4.41. We've stated part ii) of Proposition 4.40 in the form in which it is most clearly proved. When it comes up, it is needed as the implication that for $n<0$,

$$
k \geq\lfloor(n+1) / g\rfloor \Longrightarrow G / H_{+} \wedge S^{k}>n .
$$

To relate these, write $m=\lfloor(n+1) / g\rfloor$, so that

$$
m+1>(n+1) / g
$$

and by part ii) of Proposition 4.40

$$
G / H_{+} \wedge S^{k} \geq(m+1) g-1>n .
$$


4.4.2. The spectral sequence. The slice spectral sequence is the spectral sequence associated to the tower of fibration $\left\{P^{n} X\right\}$, and it takes the form

$$
E_{2}^{s, t}=\pi_{t-s}^{G} P_{t}^{t} X \Longrightarrow \pi_{t-s}^{G} X .
$$

It can be regarded as a spectral sequence of Mackey functors, or of individual homotopy groups. We have chosen our indexing so that the display of the spectral sequence is in accord with the classical Adams spectral sequence: the $E_{r}^{s, t}$-term is placed in the plane in position $(t-s, s)$. The situation is depicted in Figure 1 . The differential $d_{r}$ maps $E_{r}^{s, t}$ to $E_{r}^{s+r, t+r-1}$, or in terms the display in the plane, the group in position $(t-s, s)$ to the group in position $(t-s-1, s+r)$.

The following is an immediate consequence of Proposition 4.40. As there, we write $g=|G|$.

Theorem 4.42. Let $X$ be a $G$-spectrum. The Mackey functor homotopy groups of $P^{n} X$ satisfy

$$
\underline{\pi}_{k} P^{n} X=0 \text { for } \begin{cases}k>n & \text { if } n \geq 0 \\ k \geq\lfloor(n+1) / g\rfloor & \text { if } n<0\end{cases}
$$

and the map $X \rightarrow P^{n} X$ induces an isomorphism

$$
\underline{\pi}_{k} X \stackrel{\approx}{\rightarrow} \underline{\pi}_{k} P^{n} X \text { for } \begin{cases}k<\lfloor(n+1) / g\rfloor & \text { if } n \geq 0 \\ k \leq n & \text { if } n<0 .\end{cases}
$$

Thus for any $X$,

$$
\underset{n}{\lim _{n}} P^{n} X
$$

is contractible, the map

$$
X \rightarrow \underset{n}{\lim _{n}} P^{n} X
$$

is a weak equivalence, and for each $k$, the map

$$
\left\{\underline{\pi}_{k}(X)\right\} \rightarrow\left\{\underline{\pi}_{k} P^{n} X\right\}
$$

from the constant tower to the slice tower of Mackey functors is a pro-isomorphism.

Corollary 4.43. If $M$ is an $n$-slice then

$$
\underline{\pi}_{k} M=0
$$

if $n \geq 0$ and $k$ lies outside of the region $\lfloor n / g\rfloor \leq k \leq n$, or if $n<0$ and $k$ lies outside of the region $n \leq k<\lfloor(n+1) / g\rfloor$.

Theorem 4.42 gives the strong convergence of the slice spectral sequence, while Corollary 4.43 shows that the $E_{2}$-term vanishes outside of a restricted range of dimensions. The situation is depicted in Figure 1. The homotopy groups of individual slices lie along lines of slope -1 , and the groups contributing to $\underline{\pi}_{*} P^{n} X$ lie to the left of a line of slope -1 intersecting the $(t-s)$-axis at $(t-s)=n$. All of the groups outside the gray region are zero. The vanishing in the regions labeled 1-4 correspond to the four parts of Proposition 4.40.

Proposition 4.40 also gives a relationship between the Postnikov tower and the slice tower.

Corollary 4.44. If $X$ is an $(n-1)$-connected $G$-spectrum with $n \geq 0$ then $X \geq n$. 


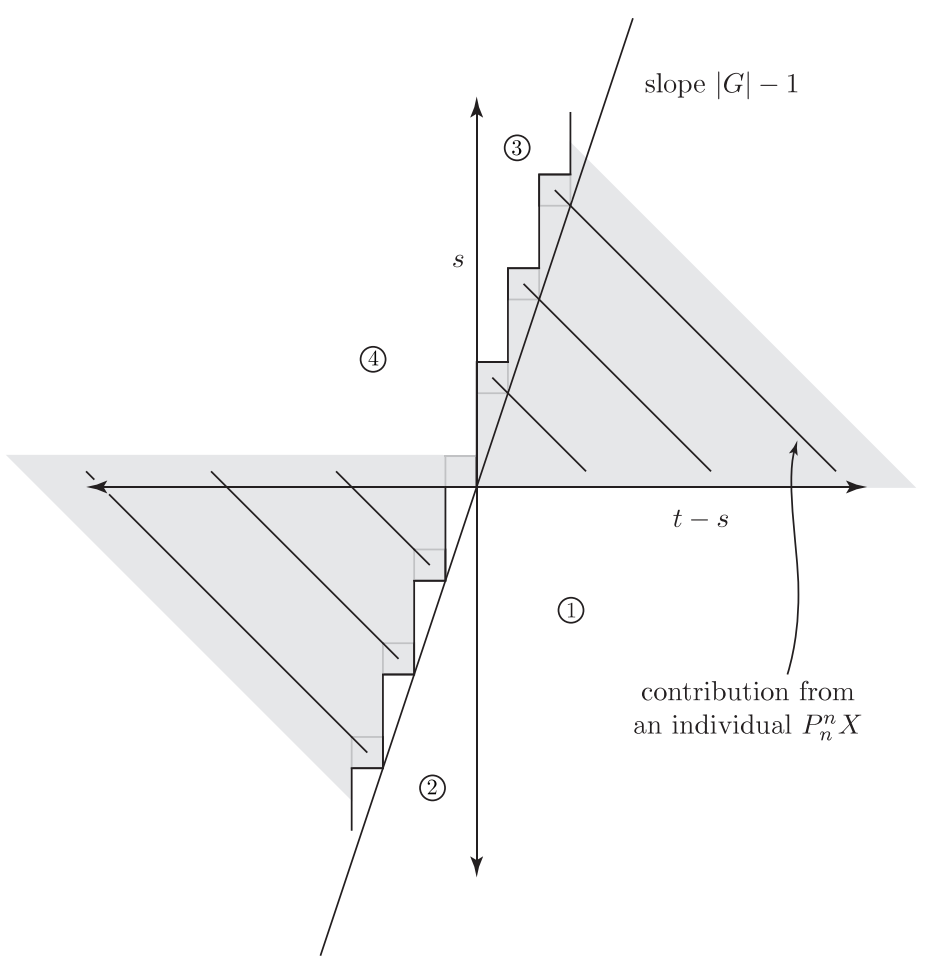

Figure 1. The slice spectral sequence

Proof: The assumption on $X$ means it is weakly equivalent to a $G$-CW spectrum having $G$-cells $G / H_{+} \wedge S^{m}$ only in dimensions $m \geq n$. By part i) of Proposition 4.40 these are in $\mathcal{S}_{\geq n}^{G}$.

We end this section with an application. The next result says that if a tower looks like the slice tower, then it is the slice tower.

Proposition 4.45. Suppose that $X \rightarrow\left\{\tilde{P}^{n}\right\}$ is a map from $X$ to a tower of fibrations with the properties

i) the map $X \rightarrow \lim _{\longleftarrow} \tilde{P}^{n}$ is a weak equivalence;

ii) the spectrum $\lim _{\longrightarrow} \tilde{P}^{n}$ is contractible;

iii) for all $n$, the fiber of the map $\tilde{P}^{n} \rightarrow \tilde{P}^{n-1}$ is an $n$-slice.

Then $\tilde{P}^{n}$ is the slice tower of $X$.

Proof: We first show that $\tilde{P}^{n}$ is slice $(n+1)$-null. We will use the criteria of Lemma 4.14. Suppose that $\widehat{S}$ is a slice cell with $\operatorname{dim} \widehat{S}>n$. By condition iii), the maps

$$
\left[\widehat{S}, \tilde{P}^{n}\right]^{G} \rightarrow\left[\widehat{S}, \tilde{P}^{n-1}\right]^{G} \rightarrow\left[\widehat{S}, \tilde{P}^{n-2}\right]^{G} \rightarrow \cdots
$$


are all monomorphisms. Since $\widehat{S}$ is finite, the map

$$
\underset{k \leq n}{\lim }\left[\widehat{S}, \tilde{P}^{k}\right]^{G} \rightarrow\left[\widehat{S}, \underset{k \leq n}{\lim _{k \leq n}} \tilde{P}^{k}\right]^{G}
$$

is an isomorphism. It then follows from assumption ii) that $\left[\widehat{S}, \tilde{P}^{n}\right]^{G}=0$. This shows that $\tilde{P}^{n}$ is slice $(n+1)$-null. Now let $\tilde{P}_{n+1}$ be the homotopy fiber of the map $X \rightarrow \tilde{P}^{n}$. By Lemma 4.16, the result will follow if we can show $\tilde{P}_{n+1}>n$. By assumption iii), for any $N>n+1$, the spectrum

$$
\tilde{P}_{n+1} \cup C \tilde{P}_{N}
$$

admits a finite filtration whose layers are $m$-slices, with $m \geq n+1$. It follows that

$$
\tilde{P}_{n+1} \cup C \tilde{P}_{N}>n \text {. }
$$

In view of the cofibration sequence

$$
\tilde{P}_{N} \rightarrow \tilde{P}_{n+1} \rightarrow \tilde{P}_{n+1} \cup C \tilde{P}_{N},
$$

to show that $\tilde{P}_{n+1}>n$ it suffices to show that $\tilde{P}_{N}>n$ for some $N>n$.

Let $Z$ be any slice $(n+1)$-null spectrum. We need to show that the $G$-space

$$
\underline{\mathfrak{S}}_{G}\left(\tilde{P}_{N}, Z\right)
$$

is contractible. We do this by studying the Mackey functor homotopy groups of the spectra involved, and appealing to an argument using the usual equivariant notion of connectivity. By Theorem 4.42, there is an integer $m$ with the property that for $k>m$,

$$
\underline{\pi}_{k} Z=0 .
$$

By Corollary 4.43 and assumption iii), for $N \gg 0$ and any $N^{\prime}>N$,

$$
\underline{\pi}_{k} \tilde{P}_{N} \cup C \tilde{P}_{N^{\prime}}=0, \quad k \leq m,
$$

so

$$
\underline{\pi}_{k} \tilde{P}_{N^{\prime}} \rightarrow \underline{\pi}_{k} \tilde{P}_{N}
$$

is an isomorphism for $k \leq m$. Since holim $\longleftarrow_{N^{\prime}} \tilde{P}_{N^{\prime}}$ is contractible this implies that for $N \gg 0$

$$
\underline{\pi}_{k} \tilde{P}_{N}=0, \quad k \leq m .
$$

Thus for $N \gg 0, \tilde{P}_{N}$ is $m$-connected in the usual sense and so

$$
\underline{\mathfrak{S}}_{G}\left(\tilde{P}_{N}, Z\right)
$$

is contractible.

4.5. The $R O(G)$-graded slice spectral sequence. Applying $R O(G)$-graded homotopy groups to the slice tower leads to an $R O(G)$-graded slice spectral sequence

$$
E_{2}^{s, V}=\pi_{V-s}^{G} P_{\operatorname{dim} V}^{\operatorname{dim} V} X \Longrightarrow \pi_{V-s}^{G} X
$$

The grading convention is chosen so that it restricts to the one of $\S 4.4 .2$ when $V$ is a trivial virtual representation. The $r^{\text {th }}$ differential is a map

$$
d_{r}: E_{2}^{s, V} \rightarrow E_{2}^{s+r, V+(r-1)} .
$$

The $R O(G)$-graded slice spectral sequence is a sum of spectral sequences, one for each element of $R O(G) / \mathbb{Z}$. We will call the spectral sequence corresponding to the coset $V+\mathbb{Z} \in R O(G) / \mathbb{Z}$ the slice spectral sequence for $\pi_{V+*}^{G} X$. This spectral sequence can be displayed on the $(x, y)$-plane, and we will do so following Adams 
conventions, with the term $E_{2}^{s, V+t}$ displayed at a position with $x$-coordinate $(V+$ $t-s)$ and $y$-coordinate $s$. For an example, see Figures 2, 3 and 4 in $\S 9$.

4.6. Special slices. In this section we investigate special slices of spectra, and introduce the notion of a spectrum with cellular slices, and of a pure $G$-spectrum. Our main result (Proposition 4.59) asserts that a map $X \rightarrow Y$ of $G$-spectra with cellular slices is a weak equivalence if and only if the underlying map of nonequivariant spectra is. This result plays an important role in the proof of the Reduction Theorem in $\S 7$. We also include material useful for investigating the slices of more general spectra.

4.6.1. Slice positive spectra, 0 -slices and (-1)-slices. In this section we will describe methods for determining the slices of spectra, and introduce a convenient class of equivariant spectra. Our first results make use of the isotropy separation sequence (§2.5.2) obtained by smashing with the cofibration sequence of pointed $G$-spaces

$$
E \mathcal{P}_{+} \rightarrow S^{0} \rightarrow \tilde{E} \mathcal{P} \text {. }
$$

The space $E \mathcal{P}_{+}$is an equivariant $\mathrm{CW}$-complex built from $G$-cells of the form $(G / H)_{+} \wedge S^{n}$ with $H \subset G$ a proper subgroup. It follows that if $W$ is a pointed $G$ space whose $H$-fixed points are contractible for all proper $H \subset G$, then $\mathcal{I}_{G}\left(E \mathcal{P}_{+}, W\right)$ is contractible.

Lemma 4.46. Fix an integer $d$. If $X$ is a $G$-spectrum with the property that $i_{H}^{*} X>d$ for all proper $H \subset G$, then $E \mathcal{P}_{+} \wedge X>d$.

Proof: Suppose that $Z \leq d$. Then

$$
\underline{\mathfrak{S}}_{G}\left(E \mathcal{P}_{+} \wedge X, Z\right) \approx \underline{\mathcal{T}}_{G}\left(E \mathcal{P}_{+}, \underline{\mathfrak{S}}_{G}(X, Z)\right) .
$$

By the assumption on $X$, the $G$-space $\underline{\mathfrak{S}}_{G}(X, Z)$ has contractible $H$ fixed points for all proper $H \subset G$. The Lemma now follows from the remark preceding its statement.

Lemma 4.47. Write $g=|G|$. The suspension spectrum of $\tilde{E} \mathcal{P}$ is in $\mathcal{S}_{\geq g-1}^{G}$.

Proof: The map $\tilde{E} \mathcal{P} \wedge S^{0} \rightarrow \tilde{E} \mathcal{P} \wedge S^{\rho_{G}-1}$ is a weak equivalence (Proposition 2.45 and Remark 2.49). The suspension spectrum of $\tilde{E} \mathcal{P}$ is in $\mathcal{S}_{\geq 0}^{G}$, since it is (-1)connected (Proposition 4.11). So $\tilde{E} \mathcal{P} \wedge S^{\rho_{G}-1} \geq g-1$ by Proposition 4.26.

Proposition 4.48. A $G$-spectrum $X$ is slice positive if and only if it is $(-1)$ connected and $\pi_{0}^{u} X=0$ (i.e., the non-equivariant spectrum $i_{0}^{*} X$ underlying $X$ is 0 connected).

Proof: The only if assertion follows from the fact that the slice cells of positive dimension are $(-1)$-connected and have 0 -connected underlying spectra. The "if" assertion is proved by induction on $|G|$, the case of the trivial group being trivial. For the induction step we may assume $X$ is $(-1)$-connected and has the property that $i_{H}^{*} X>0$ for all proper $H \subset G$. Consider the isotropy separation sequence for $X$

$$
E \mathcal{P}_{+} \wedge X \rightarrow X \rightarrow \tilde{E} \mathcal{P} \wedge X
$$


The leftmost term is slice-positive by Lemma 4.46, and the rightmost term is by Propositions 4.11 and 4.26, and Lemma 4.47. It follows that $X$ is slice-positive.

Example 4.49. Suppose that $f: S \rightarrow S^{\prime}$ is a surjective map of $G$-sets. Proposition 4.48 implies that the suspension spectrum of the mapping cone of $f$ is slice positive. This implies that if $H \underline{M}$ is an Eilenberg-MacLane spectrum which is a zero slice then for every surjective $S \rightarrow S^{\prime}$ the map $\underline{M}\left(S^{\prime}\right) \rightarrow \underline{M}(S)$ is a monomorphism. The proposition below shows that this is also a sufficient condition.

Proposition 4.50. i) A spectrum $X$ is a (-1)-slice if and only if it is of the form $X=\Sigma^{-1} H \underline{M}$, with $\underline{M}$ an arbitrary Mackey functor.

ii) A spectrum $X$ is a 0 -slice if and only if it is of the form $H \underline{M}$ with $\underline{M}$ a Mackey functor all of whose restriction maps are monomorphisms.

Remark 4.51. The condition on $\underline{M}$ in ii) is that if $S \rightarrow S^{\prime}$ is a surjective map of finite $G$-sets then $\underline{M}\left(S^{\prime}\right) \rightarrow \underline{M}(S)$ is a monomorphism. Let $G$ act on $G \times S$ and $G \times S^{\prime}$ through its left action on $G$. Then $G \times S \rightarrow G \times S^{\prime}$ has a section, so $\underline{M}\left(G \times S^{\prime}\right) \rightarrow \underline{M}(G \times S)$ is always a monomorphism. Using this one easily checks that condition is also equivalent to requiring that for every finite $G$-set $S^{\prime}$, the map $\underline{M}\left(S^{\prime}\right) \rightarrow \underline{M}\left(G \times S^{\prime}\right)$, induced by the action mapping $G \times S^{\prime} \rightarrow S^{\prime}$, is a monomorphism.

Proof: The first assertion is immediate from Proposition 4.20, which, combined with part i) of Proposition 4.40, also shows that a 0-slice is an Eilenberg-MacLane spectrum. Example 4.49 gives the "only if" part of the second assertion. For the "if" part, suppose that $\underline{M}$ is a Mackey functor all of whose restrictions maps are monomorphisms, and consider the sequence

$$
P_{1} H \underline{M} \rightarrow H \underline{M} \rightarrow P^{0} H \underline{M} .
$$

Since $P_{1} H \underline{M} \geq 0$ it is $(-1)$-connected, and so $P_{1} H \underline{M}$ is an Eilenberg-MacLane spectrum. For convenience, write

$$
\begin{aligned}
\underline{M}^{\prime} & =\underline{\pi}_{0} P_{1} H \underline{M} \\
\underline{M}^{\prime \prime} & =\underline{\pi}_{0} P^{0} H \underline{M}
\end{aligned}
$$

so that there is a short exact sequence

$$
\underline{M}^{\prime} \longmapsto \underline{M} \rightarrow \underline{M}^{\prime \prime} .
$$

Suppose that $S$ is any finite $G$-set and consider the following diagram

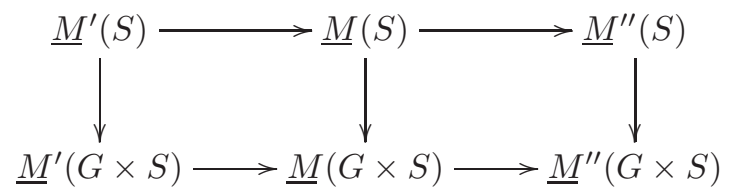

in which the rows are short exact, and the vertical maps are induced by the action mapping, as in Remark 4.51. The bottom right arrow is an isomorphism since $i_{0}^{*} H \underline{M} \rightarrow i_{0}^{*} P^{0} H \underline{M}$ is an equivalence. Thus $\underline{M}^{\prime}(G \times S)=0$ (this also follows from Proposition 4.48). The claim now follows from a simple diagram chase.

Remark 4.52. The second assertion of Proposition 4.50 can also be deduced directly from Corollary 3.6. 
Corollary 4.53. If $X=H \underline{M}$ is a zero slice and $\pi_{0}^{u} X=0$ then $X$ is contractible.

Corollary 4.54. The (-1)-slice of $S^{-1}$ is $\Sigma^{-1} H \underline{A}$. The zero slice of $S^{0}$ is $H \underline{\mathbb{Z}}$.

Proof: The first assertion follows easily from Part i) of Proposition 4.50. For the second assertion note that the $S^{0} \rightarrow H \underline{A}$ is a $P^{0}$-equivalence, so the zero slice of $S^{0}$ is $P^{0} H \underline{A}$. Consider the fibration sequence

$$
H \underline{I} \rightarrow H \underline{A} \rightarrow H \underline{\mathbb{Z}}
$$

in which $\underline{I}=\operatorname{ker} \underline{A} \rightarrow \underline{\mathbb{Z}}$ is the augmentation ideal. The leftmost term is slice positive by Proposition 4.48 , and the rightmost term is in $\mathcal{S}_{\leq 0}^{G}$ by Proposition 4.50. The claim now follows from Lemma 4.16.

Corollary 4.55. For $K \subset G$, the $m|K|$-slice of $\widehat{S}(m, K)$ is

$$
H \underline{\mathbb{Z}} \wedge \widehat{S}(m, K)
$$

and the $(m|K|-1)$-slice of $\Sigma^{-1} \widehat{S}(m, K)$ is

$$
H \underline{A} \wedge \Sigma^{-1} \widehat{S}(m, K) \text {. }
$$

Proof: Using the fact that $G_{+}{ }_{K}(-)$ commutes with the formation of the slice tower (Proposition 4.21) it suffices to consider the case $K=G$. The result then follows from Corollaries 4.25 and 4.54.

4.6.2. Cellular slices, isotropic and pure spectra.

Definition 4.56. A $d$-slice is cellular if it is of the form $H \underline{\mathbb{Z}} \wedge \widehat{W}$, where $\widehat{W}$ is a wedge of slice cells of dimension $d$. A cellular slice is isotropic if $\widehat{W}$ can be written as a wedge of slice cells, none of which is free (i.e., of the form $G_{+} \wedge S^{n}$ ). A cellular slice is pure if $\widehat{W}$ can be written as a wedge of regular slice cells (those of the form $\widehat{S}(m, K)$, and not $\left.\Sigma^{-1} \widehat{S}(m, K)\right)$.

Definition 4.57. A $G$-spectrum $X$ has cellular slices if $P_{n}^{n} X$ is cellular for all $d$, and is isotropic or pure if its slices are isotropic or pure.

Lemma 4.58. Suppose that $f: X \rightarrow Y$ is a map of cellular $d$-slices and $\pi_{d}^{u} f$ is an isomorphism. Then $f$ is a weak equivalence.

Proof: The proof is by induction on $|G|$. If $G$ is the trivial group, the result is obvious since $X$ and $Y$ are Eilenberg-MacLane spectra. Now suppose we know the result for all proper $H \subset G$, and consider the map of isotropy separation sequences

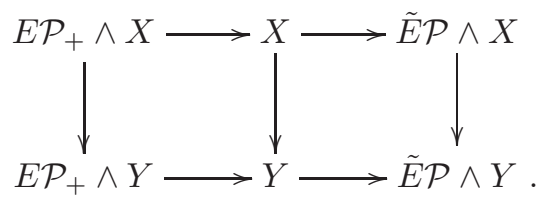

By the induction hypothesis, the left vertical map is a weak equivalence. If $d$ is not congruent to 0 or -1 modulo $|G|$ then the rightmost terms are contractible, since every slice cell of dimension $d$ is induced. Smashing with $S^{m \rho_{G}}$ for suitable $m$, we 
may therefore assume $d=0$ or $d=-1$. Smashing with $S^{1}$ in case $d=-1$ we reduce to the case $d=0$ and therefore assume that $X=H \underline{M}_{0}$ and $Y=H \underline{M}_{1}$ with $\underline{M}_{0}$ and $\underline{M}_{1}$ permutation Mackey functors. The result then follows from part iv) of Lemma 3.3.

Proposition 4.59. Suppose that $X$ and $Y$ have cellular slices. If $f: X \rightarrow Y$ has the property that $\pi_{*}^{u} f$ is an isomorphism. Then $f$ is a weak equivalence.

Proof: It suffices to show that for each $d$ the induced map of slices

$$
P_{d}^{d} X \rightarrow P_{d}^{d} Y
$$

is a weak equivalence. Since the map of ordinary spectra underlying the slice tower is the Postnikov tower, the map satisfies the conditions of Lemma 4.58, and the result follows.

For certain slices, the condition on $Y$ in Proposition 4.59 can be dropped.

Lemma 4.61. Suppose that $f: X \rightarrow Y$ is a map of 0 -slices and $X$ is cellular. If $\pi_{0}^{u} f$ is an isomorphism then $f$ is an equivalence.

Proof: Write $X=H \underline{M}$ and $Y=H \underline{M}^{\prime}$, and let $S$ be a finite $G$-set. Consider the diagram

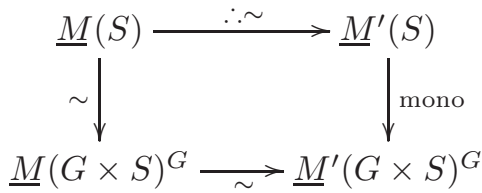

in which the vertical maps come from the action mapping $G \times S \rightarrow S$ (see the discussion preceding Lemma 3.3). The bottom arrow is an isomorphism by assumption. The vertical maps are monomorphisms by Proposition 4.50. The left vertical map is an isomorphism since $\underline{M}$ is a permutation Mackey functor (part ii) of Lemma 3.3). The result follows.

Proposition 4.62. Suppose that $f: X \rightarrow Y$ is a map of d-slices, $X$ is cellular and $d \not \equiv-1 \bmod p$ for any prime $p$ dividing $|G|$. If $\pi_{d}^{u} X \rightarrow \pi_{d}^{u} Y$ is an isomorphism then $f$ is a weak equivalence.

Proof: Let $C$ be the mapping cone of $f$. We know that $C \geq d$. We will show that

$$
[\widehat{S}, C]^{G}=0
$$

for all slice cells $\widehat{S}$ with $\operatorname{dim} \widehat{S} \geq d$. This will show (Lemma 4.14) that $C<d$ and hence must be contractible since its identity map is null. The assertion is obvious when $G$ is the trivial group. By induction on $|G|$ we may assume $\widehat{S}$ is not induced. If $d$ is divisible by $|G|$ we may smash with $S^{-d /|G| \rho_{G}}$ and reduce to the case $d=0$ which is Lemma 4.61. It remains to show that $\pi_{m \rho_{G}}^{G} C=0$ when $m|G| \geq d$ and that $\pi_{m \rho_{G}-1}^{G} C=0$ when $m|G|-1 \geq d$. Since

$$
d \not \equiv 0,-1 \quad \bmod |G|,
$$


the conditions in fact implies $m|G|-1>d$. So we are in the situation $m|G|-1>d$ and we need to show that both $\pi_{m \rho_{G}}^{G} C$ and $\pi_{m \rho_{G}-1}^{G} C$ are zero. The exact sequence

$$
\pi_{m \rho_{G}}^{G} Y \rightarrow \pi_{m \rho_{G}}^{G} C \rightarrow \pi_{m \rho_{G}-1}^{G} X
$$

gives the vanishing of $\pi_{m \rho_{G}}^{G} C$. For the remaining case consider the exact sequence

$$
\pi_{m \rho_{G}-1}^{G} Y \rightarrow \pi_{m \rho_{G}-1}^{G} C \rightarrow \pi_{m \rho_{G}-2}^{G} X \rightarrow \pi_{m \rho_{G}-2}^{G} Y .
$$

As above, the left group vanishes since $Y$ is a $d$-slice and $S^{m \rho_{G}-1}>d$. Lemma 4.63 below implies that the left vertical map in

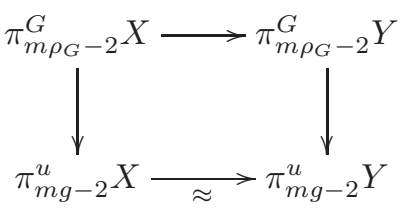

is monomorphism, and therefore so is the top horizontal map. Thus $\pi_{m \rho_{G}-1}^{G} C=0$ by exactness.

Lemma 4.63. Suppose $\widehat{S}$ is a slice cell of dimension $d$. If $m|G|-1>d$ then the restriction mapping

is a monomorphism.

$$
\pi_{m \rho_{G}-2}^{G} H \underline{\mathbb{Z}} \wedge \widehat{S} \rightarrow \pi_{m g-2}^{u} H \underline{\mathbb{Z}} \wedge \widehat{S}
$$

Proof: When $G$ is trivial the map is an isomorphism. By induction on $|G|$ we may therefore assume $G$ is not the trivial group and that $\widehat{S}$ is not induced, in which case $\widehat{S}=S^{k \rho_{G}}$ or $\widehat{S}=S^{k \rho_{G}-1}$. Note that

$$
S^{m \rho_{G}-2}=S^{(m-1) \rho_{G}-1} \wedge S^{\rho_{G}-1} \geq(m-1)|G|-1>(m-2)|G|
$$

so that both $\pi_{m \rho_{G}-2}^{G} H \underline{\mathbb{Z}} \wedge S^{k \rho_{G}}$ and $\pi_{m \rho_{G}-2}^{G} H \underline{\mathbb{Z}} \wedge S^{k \rho_{G}-1}$ are zero unless $k=m-1$. The group $\pi_{m \rho_{G}-2}^{G} H \underline{\mathbb{Z}} \wedge S^{(m-1) \rho_{G}-1}$ is zero since it is isomorphic to

$$
\pi_{m \rho_{G}-1}^{G} H \underline{\mathbb{Z}} \wedge S^{(m-1) \rho_{G}}
$$

and $S^{m \rho_{G}-1} \geq m|G|-1>(m-1)|G|$. This leaves the group

$$
\pi_{m \rho_{G}-2}^{G} H \underline{\mathbb{Z}} \wedge S^{(m-1) \rho_{g}} \approx \pi_{\rho_{G}-2}^{G} H \underline{\mathbb{Z}}
$$

whose triviality was established in Example 3.19.

4.6.3. The special case in which $G$ is a finite 2-group. In this section we record some results which are special to the case in which $G$ has order a power of 2 . The results about even slices are used in the proof of the Reduction Theorem in $\S 7.2$. The results on odd slices were used in an earlier approach to the main results of this paper, but are no longer. We include them here because they provide useful tools for investigating slices of various spectra. Throughout this section the group $G$ will be a finite 2-group.

Suppose that $X$ is a $G$-spectrum with the property that $\pi_{d}^{u} X$ is a free abelian group. In $\S 5.3$ we will define a refinement of $\pi_{d}^{u} X$ to be a map

$$
c: \widehat{W} \rightarrow X
$$


in which $\widehat{W}$ is a wedge of slice cells of dimension $d$, with the property that the map $\pi_{d}^{u} \widehat{W} \rightarrow \pi_{d}^{u} X$ is an isomorphism.

Proposition 4.64. If $\widehat{W} \rightarrow X$ is a refinement of $\pi_{2 k}^{u} X$ then the canonical map

$$
H \underline{\mathbb{Z}} \wedge \widehat{W} \rightarrow P_{2 k}^{2 k} X
$$

is an equivalence.

Proof: By Corollary 4.55 (and the fact that the formation of slices commutes with the formation of wedges), the map

$$
\widehat{W} \rightarrow H \underline{\mathbb{Z}} \wedge \widehat{W}
$$

induces an equivalence

$$
P_{2 k}^{2 k} \widehat{W} \rightarrow H \underline{\mathbb{Z}} \wedge \widehat{W}
$$

Applying $P_{2 k}^{2 k}$ to $\widehat{W} \rightarrow X$ then leads to a map

$$
H \underline{\mathbb{Z}} \wedge \widehat{W} \rightarrow P_{2 k}^{2 k} X
$$

which, since the slice tower refines the Postnikov tower, is an equivalence of underlying non-equivariant spectra. The result now follows from Proposition 4.62, since the only prime dividing $|G|$ is 2 .

Proposition 4.64 gives some control over the even slices of a $G$-spectrum $X$ when $G$ is a 2-group. The odd slices are something of a different story, and getting at them requires some knowledge of the equivariant homotopy type of $X$. Note that by Proposition 4.50 any Mackey functor can occur in an odd slice. On the other hand, only special ones can occur in even slices.

Corollary 4.65. If $\widehat{S}$ is a slice cell of odd dimension $d$, then for any $X$,

$$
[\widehat{S}, X]^{G} \approx\left[\widehat{S}, P_{d}^{d} X\right]^{G} \text {. }
$$

Proof: Since the formation of $P_{d}^{d} X$ commutes with the functors $i_{H}^{*}$, induction on $|G|$ reduces us to the case when $\widehat{S}$ is not an induced slice cell. So we may assume $\widehat{S}=S^{m \rho_{G}-1}$. Smashing $\widehat{S}$ and $X$ with $S^{-m \rho_{G}}$, and using Corollary 4.25 reduces to the case $m=0$, which is given by Proposition 4.20.

The situation most of interest to us in this paper is when the odd slices are contractible. Proposition 4.66 below gives a useful criterion.

Proposition 4.66. For a $G$ spectrum $X$ and an odd integer $d$, the following are equivalent:

i) The d-slice of $X$ is contractible;

ii) For every slice cell $\widehat{S}$ of dimension $d,[\widehat{S}, X]^{G}=0$.

Proof: By Corollary 4.65 (which requires $d$ to be odd), there is an isomorphism

$$
[\widehat{S}, X]^{G}=\left[\widehat{S}, P_{d}^{d} X\right]^{G} .
$$

By Lemma 4.14, the vanishing of this group implies that $P_{d}^{d} X<d$ and hence must be contractible, since it is also $\geq d$. 
Corollary 4.67. Suppose that $d$ is odd. If $X \rightarrow Y \rightarrow Z$ is a cofibration sequence, and the $d$-slices of $X$ and $Z$ are contractible, then the $d$-slice of $Y$ is contractible.

Proof: This is immediate from Proposition 4.66 and the long exact sequence of homotopy classes of maps.

Remark 4.68. Using the slice spectral sequence one can easily show that a pure spectrum always admits a refinement of homotopy groups. Thus the results above say that a spectrum $X$ is pure if and only if the even homotopy groups admit an equivariant refinement, and the "slice homotopy groups" $\pi_{m \rho_{H}-1}^{H} X$ are all zero whenever $H \subset G$ is non-trivial.

4.7. Further multiplicative properties of the slice filtration. In this section we show that the slice filtration has the expected multiplicative properties for pure spectra. Our main result is Proposition 4.69 below. It has the consequence that if $X$ and $Y$ are pure spectra, and $E_{r}^{s, t}(-)$ is the slice spectral sequence, then there is a map of spectral sequences

$$
E_{r}^{s, t}(X) \otimes E_{r}^{s^{\prime}, t^{\prime}}(Y) \rightarrow E_{r}^{s+s^{\prime}, t+t^{\prime}}(X \wedge Y)
$$

representing the pairing $\underline{\pi}_{*} X \wedge \underline{\pi}_{*} Y \rightarrow \underline{\pi}_{*}(X \wedge Y)$. In other words, multiplication in the slice spectral sequence of pure spectra behaves in the expected manner. We leave the deduction of this property from Proposition 4.69 to the reader.

Proposition 4.69. If $X \geq n$ is pure and $Y \geq m$ has cellular slices, then $X \wedge Y \geq$ $n+m$.

Proof: We need to show that $P^{n+m-1}(X \wedge Y)$ is contractible. By Lemma 4.30 the map

$$
X \wedge Y \rightarrow P^{n+m-1} X \wedge P^{n+m-1} Y
$$

is a $P^{n+m-1}$-equivalence, so we may reduce to the case in which the slice filtrations of $X$ and $Y$ are finite. That case in turn reduces to the situation in which

$$
\begin{aligned}
& X=H \underline{\mathbb{Z}} \wedge \widehat{S}(m, K) \\
& Y=H \underline{\mathbb{Z}} \wedge \widehat{S}^{\prime}
\end{aligned}
$$

in which $\widehat{S}^{\prime}$ is any slice cell. By induction on $|G|$ the assertion further reduces to the case in which neither $\widehat{S}$ nor $\widehat{S}^{\prime}$ is induced. Thus we may assume

$$
\begin{aligned}
& X=H \underline{\mathbb{Z}} \wedge S^{k \rho_{G}} \\
& Y=H \underline{\mathbb{Z}} \wedge S^{\ell \rho_{G}} \quad \text { or } \quad H \underline{\mathbb{Z}} \wedge \Sigma^{-1} S^{\ell \rho_{G}},
\end{aligned}
$$

in which case the result follows from Proposition 4.26.

\section{The COMPLEX COBORDISM SPECTRUM}

From here forward we specialize to the case $G=C_{2^{n}}$, and for convenience localize all spectra at the prime 2 . Write

$$
g=|G|,
$$

and let $\gamma \in G$ be a fixed generator. 
5.1. The spectrum $M U^{((G))}$. We now introduce our equivariant variation on the complex cobordism spectrum by defining

$$
M U^{((G))}=N_{C_{2}}^{G} M U_{\mathbb{R}}
$$

where $M U_{\mathbb{R}}$ is the $C_{2}$-equivariant real bordism spectrum of Landweber [43] and Fujii [24] (and further studied by Araki [6] and Hu-Kriz [35]). In §B.12 we will give a construction of $M U_{\mathbb{R}}$ as a commutative algebra in $\mathcal{S}^{C_{2}}$. The norm is taken along the unique inclusion $C_{2} \subset G$. Since the norm is symmetric monoidal, and its left derived functor may be computed on the spectra underlying cofibrant commutative rings (Proposition B.147), the spectrum $M U^{((G))}$ is an equivariant commutative ring spectrum. For $H \subset G$ the unit of the restriction-norm adjunction (Proposition 2.27) gives a canonical commutative algebra map

$$
M U^{((H))} \rightarrow i_{H}^{*} M U^{((G))} .
$$

By analogy with the shorthand $i_{0}^{*}$ for restriction along the inclusion of the trivial group, we will employ the shorthand notation

$$
i_{1}^{*}=i_{C_{2}}^{*}
$$

for the restriction map $\mathcal{S}^{G} \rightarrow \mathcal{S}^{C_{2}}$ induced by the unique inclusion $C_{2} \subset G$. Restricting, one has a $C_{2}$-equivariant smash product decomposition

$$
i_{1}^{*} M U^{((G))}=\bigwedge_{j=0}^{g / 2-1} \gamma^{j} M U_{\mathbb{R}} .
$$

5.2. Real bordism, real orientations and formal groups. We begin by reviewing work of Araki [6] and Hu-Kriz [35] on real bordism.

5.2.1. The formal group. Consider $\mathbf{C P}^{n}$ and $\mathbf{C P}^{\infty}$ as pointed $C_{2}$-spaces under the action of complex conjugation, with $\mathbf{C P}^{0}$ as the base point. The fixed point spaces are $\mathbf{R} \mathbf{P}^{n}$ and $\mathbf{R} \mathbf{P}^{\infty}$. There are homeomorphisms

$$
\mathbf{C P}^{n} / \mathbf{C P}^{n-1} \equiv S^{n \rho_{2}}
$$

and in particular an identification $\mathbf{C P}^{1} \equiv S^{\rho_{2}}$.

Definition 5.4 (Araki [6]). Let $E$ be a $C_{2}$-equivariant homotopy commutative ring spectrum. A real orientation of $E$ is a class $\bar{x} \in \tilde{E}_{C_{2}}^{\rho_{2}}\left(\mathbf{C P}^{\infty}\right)$ whose restriction to

$$
\tilde{E}_{C_{2}}^{\rho_{2}}\left(\mathbf{C P}^{1}\right)=\tilde{E}_{C_{2}}^{\rho_{2}}\left(S^{\rho_{2}}\right) \approx E_{C_{2}}^{0}(\mathrm{pt})
$$

is the unit. A real oriented spectrum is a $C_{2}$-equivariant ring spectrum $E$ equipped with a real orientation.

If $(E, \bar{x})$ is a real oriented spectrum and $f: E \rightarrow E^{\prime}$ is an equivariant multiplicative map, then

$$
f_{*}(\bar{x}) \in\left(E^{\prime}\right)^{\rho_{2}}\left(\mathbf{C P}^{\infty}\right)
$$

is a real orientation of $E^{\prime}$. We will often not distinguish in notation between $\bar{x}$ and $f_{*} \bar{x}$.

Example 5.5. The zero section $\mathbf{C P}^{\infty} \rightarrow M U(1)$ is an equivariant equivalence, and defines a real orientation

$$
\bar{x} \in M U_{\mathbb{R}}^{\rho_{2}}\left(\mathbf{C P}^{\infty}\right),
$$

making $M U_{\mathbb{R}}$ into a real oriented spectrum. 
Example 5.6. From the map

$$
M U_{\mathbb{R}} \rightarrow i_{1}^{*} M U^{((G))}
$$

provided by (5.1), the spectrum $i_{1}^{*} M U^{((G))}$ gets a real orientation which we'll also denote

$$
\bar{x} \in\left(M U^{((G))}\right)^{\rho_{2}}\left(\mathbf{C P}^{\infty}\right) .
$$

Example 5.7. If $\left(H, \bar{x}_{H}\right)$ and $\left(E, \bar{x}_{E}\right)$ are two real oriented spectra then $H \wedge E$ has two real orientations given by

$$
\bar{x}_{H}=\bar{x}_{H} \otimes 1 \text { and } \bar{x}_{E}=1 \otimes \bar{x}_{E} .
$$

The following result of Araki follows easily from the homeomorphisms (5.3).

Theorem 5.8 (Araki [6]). Let $E$ be a real oriented cohomology theory. There are isomorphisms

$$
\begin{aligned}
E^{\star}\left(\mathbf{C P}^{\infty}\right) & \approx E^{\star}[[\bar{x}]] \\
E^{\star}\left(\mathbf{C P}^{\infty} \times \mathbf{C P}^{\infty}\right) & \approx E^{\star}[[\bar{x} \otimes 1,1 \otimes \bar{x}]]
\end{aligned}
$$

Because of Theorem 5.8, the map $\mathbf{C P}^{\infty} \times \mathbf{C} \mathbf{P}^{\infty} \rightarrow \mathbf{C P}^{\infty}$ classifying the tensor product of the two tautological line bundles defines a formal group law over $\pi_{\star}^{G} E$. Using this, much of the theory relating formal groups, complex cobordism, and complex oriented cohomology theories works for $C_{2}$-equivariant spectra, with $M U_{\mathbb{R}}$ playing the role of $M U$. For information beyond the discussion below, see [6, 35].

Remark 5.9. A real orientation $\bar{x}$ corresponds to a coordinate on the corresponding formal group. Because of this we will use the terms interchangeably, preferring "coordinate" when the discussion predominantly concerns the formal group, and "real orientation" when it concerns spectra.

The standard formulae from the theory of formal groups give elements in the $R O\left(C_{2}\right)$-graded homotopy groups $\pi_{\star}^{C_{2}} E$ of real oriented $E$. For example, there is a map from the Lazard ring to $\pi_{\star}^{C_{2}} E$ classifying the formal group law. Using Quillen's theorem to identify the Lazard ring with the complex cobordism ring this map can be written as

$$
M U_{*} \rightarrow \pi_{\star}^{C_{2}} E .
$$

It sends $M U_{2 n}$ to $\pi_{n \rho_{2}}^{C_{2}} E$. When $E=M U_{\mathbb{R}}$ this splits the forgetful map

$$
\pi_{n \rho_{2}}^{C_{2}} M U_{\mathbb{R}} \rightarrow \pi_{2 n}^{u} M U_{\mathbb{R}}=\pi_{2 n} M U
$$

which is therefore surjective. A similar discussion applies to iterated smash products of $M U_{\mathbb{R}}$ giving

Proposition 5.11. For every $m>0$, the above construction gives a ring homomorphism

$$
\pi_{*}^{u} \bigwedge^{m} M U_{\mathbb{R}} \rightarrow \bigoplus_{j} \pi_{j \rho_{2}}^{C_{2}} \bigwedge^{m} M U_{\mathbb{R}}
$$

splitting the forgetful map

$$
\bigoplus_{j} \pi_{j \rho_{2}}^{C_{2}} \bigwedge^{m} M U_{\mathbb{R}} \rightarrow \pi_{*}^{u} \bigwedge^{m} M U_{\mathbb{R}}
$$

In particular, (5.13) is a split surjection. 
It is a result of $\mathrm{Hu}-\mathrm{Kriz}[35]$ that (5.13) is in fact an isomorphism. This result, and a generalization to $M U^{((G))}$ can be recovered from the slice spectral sequence.

The class

$$
\bar{x}_{H} \in H_{C_{2}}^{\rho_{2}}\left(\mathbf{C P}^{\infty} ; \underline{\mathbb{Z}}_{(2)}\right)
$$

corresponding to $1 \in H_{C_{2}}^{0}\left(\mathrm{pt}, \underline{\mathbb{Z}}_{(2)}\right)$ under the isomorphism

$$
H_{C_{2}}^{\rho_{2}}\left(\mathbf{C P}^{\infty} ; \underline{\mathbb{Z}}_{(2)}\right) \approx H_{C_{2}}^{\rho_{2}}\left(\mathbf{C P}^{2} ; \underline{\mathbb{Z}}_{(2)}\right) \approx H_{C_{2}}^{0}\left(\mathrm{pt}, \underline{\mathbb{Z}}_{(2)}\right)
$$

defines a real orientation of $H \underline{\mathbb{Z}}_{(2)}$. As in Example 5.7, the classes $\bar{x}$ and $\bar{x}_{H}$ give two orientations of $E=H \underline{\mathbb{Z}}_{(2)} \wedge M U_{\mathbb{R}}$. By Theorem 5.8 these are related by a power series

$$
\begin{aligned}
\bar{x}_{H} & =\log _{F}(\bar{x}) \\
& =\bar{x}+\sum_{i>0} \bar{m}_{i} \bar{x}^{i+1},
\end{aligned}
$$

with

$$
\bar{m}_{i} \in \pi_{i \rho_{2}}^{C_{2}} H \underline{\mathbb{Z}}_{(2)} \wedge M U_{\mathbb{R}}
$$

This power series is the logarithm of $F$. Similarly, the invariant differential on $F$ gives classes $(n+1) \bar{m}_{n} \in \pi_{n \rho_{2}}^{C_{2}} M U_{\mathbb{R}}$. The coefficients of the formal sum give

$$
\bar{a}_{i j} \in \pi_{(i+j-1) \rho_{2}}^{C_{2}} M U_{\mathbb{R}}
$$

Remark 5.14. Since the generator of $C_{2}$ acts by $(-1)^{n}$ on

$$
H_{2 n} i_{0}^{*} S^{n \rho_{2}}=\pi_{2 n}^{u} H \underline{\mathbb{Z}} \wedge S^{n \rho_{2}},
$$

it acts also acts by $(-1)^{n}$ on the non-equivariant class $m_{n}$ underlying $\bar{m}_{n}$ and by $(-1)^{n}$ on $\pi_{2 n}^{u} \bigwedge^{m} M U_{\mathbb{R}}=\pi_{2 n} \bigwedge^{m} M U$.

If $\left(E, \bar{x}_{E}\right)$ is a real oriented spectrum then $E \wedge M U_{\mathbb{R}}$ has two orientations

$$
\begin{aligned}
& \bar{x}_{E}=\bar{x}_{E} \otimes 1 \\
& \bar{x}_{R}=1 \otimes \bar{x} .
\end{aligned}
$$

These two orientations are related by a power series

$$
\bar{x}_{R}=\sum \bar{b}_{i} x_{E}^{i+1}
$$

defining classes

$$
\bar{b}_{i}=\bar{b}_{i}^{E} \in \pi_{i \rho_{2}}^{C_{2}} E \wedge M U_{\mathbb{R}}
$$

The power series (5.15) is an isomorphism over $\pi_{\star}^{C_{2}} E \wedge M U_{\mathbb{R}}$

$$
F_{E} \rightarrow F_{R}
$$

of the formal group law for $\left(E, \bar{x}_{E}\right)$ with the formal group law for $\left(M U_{\mathbb{R}}, \bar{x}\right)$.

Theorem 5.16 (Araki [6]). The map

$$
E_{\star}\left[\bar{b}_{1}, \bar{b}_{2}, \ldots\right] \rightarrow \pi_{\star}^{C_{2}} E \wedge M U_{\mathbb{R}}
$$

is an isomorphism. 
Araki's theorem has an evident geometric counterpart. For each $j$ choose a map

$$
S^{j \rho_{2}} \rightarrow E \wedge M U_{\mathbb{R}}
$$

representing $\bar{b}_{j}$. As in $\S 2.4$, let

$$
S\left[\bar{b}_{j}\right]=\bigvee_{k \geq 0} S^{k \cdot j \rho_{2}}
$$

be the free associative algebra on $S^{j \rho_{2}}$ and

$$
S\left[\bar{b}_{j}\right] \rightarrow E \wedge M U_{\mathbb{R}}
$$

the homotopy associative algebra map extending (5.39). Using the multiplication map, smash these together to form a map of spectra

$$
E\left[\bar{b}_{1}, \bar{b}_{2}, \ldots\right] \rightarrow E \wedge M U^{((G))},
$$

where

$$
E\left[\bar{b}_{1}, \bar{b}_{2}, \ldots\right]=E \wedge \underset{k}{\underset{k}{\longrightarrow}} S\left[\bar{b}_{1}\right] \wedge S\left[\bar{b}_{2}\right] \wedge \cdots \wedge S\left[\bar{b}_{k}\right] .
$$

The map on $R O\left(C_{2}\right)$-graded homotopy groups induced by (5.17) is the isomorphism of Araki's theorem. This proves

Corollary 5.18. If $E$ is a real oriented spectrum then there is a weak equivalence

$$
E \wedge M U_{\mathbb{R}} \approx E\left[\bar{b}_{1}, \bar{b}_{2}, \ldots\right] .
$$

Remark 5.19. If $E$ is strictly associative then (5.17) is a map of associative algebras, and the above identifies $E \wedge M U_{\mathbb{R}}$ as a twisted monoid ring over $E$.

As in $\S 2.4$, write

$$
S^{0}\left[\bar{b}_{1}, \bar{b}_{2}, \ldots\right]=\underset{\underset{k}{h o l i m}}{\longrightarrow} S^{0}\left[\bar{b}_{1}\right] \wedge S^{0}\left[\bar{b}_{2}\right] \wedge \cdots \wedge S^{0}\left[\bar{b}_{k}\right],
$$

and

$$
S^{0}\left[G \cdot \bar{b}_{1}, G \cdot \bar{b}_{1}, \ldots\right]=N_{C_{2}}^{G} S^{0}\left[\bar{b}_{1}, \bar{b}_{2}, \ldots\right] .
$$

Using Proposition 4.7 one can easily check that $S^{0}\left[G \cdot \bar{b}_{1}, G \cdot \bar{b}_{2}, \ldots\right]$ is a wedge of isotropic regular slice cells. Finally, let

$$
M U^{((G))}\left[G \cdot \bar{b}_{1}, G \cdot \bar{b}_{2}, \ldots\right]=M U^{((G))} \wedge S^{0}\left[G \cdot \bar{b}_{1}, G \cdot \bar{b}_{1}, \ldots\right]
$$

Corollary 5.20. For $H \subset G$ of index 2 , there is an equivalence of $H$-equivariant associative algebras

$$
i_{H}^{*} M U^{((G))} \approx M U^{((H))}\left[H \cdot \bar{b}_{1}, H \cdot \bar{b}_{2}, \ldots\right] .
$$

Proof: Apply $N_{C_{2}}^{H}$ to the decomposition of Corollary 5.18 with $E=M U_{\mathbb{R}}$. 
5.2.2. The unoriented cobordism ring. Passing to geometric fixed points from

$$
\bar{x}: \mathbf{C P}^{\infty} \rightarrow \Sigma^{\rho_{2}} M U_{\mathbb{R}}
$$

gives the canonical inclusion

$$
a: \mathbf{R} \mathbf{P}^{\infty}=M O(1) \rightarrow \Sigma M O,
$$

defining the $M O$ Euler class of the tautological line bundle. There are isomorphisms

$$
\begin{aligned}
M O^{*}\left(\mathbf{R P}^{\infty}\right) & \approx M O^{*}[[a] \\
M O^{*}\left(\mathbf{R P}^{\infty} \times \mathbf{R} \mathbf{P}^{\infty}\right) & \approx M O^{*}[[a \otimes 1,1 \otimes a \rrbracket
\end{aligned}
$$

and the multiplication map $\mathbf{R P}^{\infty} \times \mathbf{R} \mathbf{P}^{\infty} \rightarrow \mathbf{R} \mathbf{P}^{\infty}$ gives a formal group law over $M O_{*}$. By Quillen [67], it is the universal formal group law $F$ over a ring of characteristic 2 for which $F(a, a)=0$.

As described by Quillen [69, Page 53], the formal group can be used to give convenient generators for the unoriented cobordism ring. Let

$$
e \in H^{1}\left(\mathbf{R P}^{\infty} ; \mathbb{Z} / 2\right)
$$

be the $H \mathbb{Z} / 2$ Euler class of the tautological line bundle. Over $\pi_{*} H \mathbb{Z} / 2 \wedge M O$ there is a power series relating $e$ and the image of the class $a$

$$
e=\ell(a)=a+\sum \alpha_{n} a^{n+1}
$$

Lemma 5.21. The composite series

$$
\left(a+\sum \alpha_{2^{j}-1} a^{2^{j}}\right)^{-1} \circ \ell(a)=a+\sum_{j>0} h_{j} a^{j+1}
$$

has coefficients in $\pi_{*} M O$. The classes $h_{j}$ with $j+1=2^{k}$ are zero. The remaining $h_{j}$ are polynomial generators for the unoriented cobordism ring

$$
\pi_{*} M O=\mathbb{Z} / 2\left[h_{j}, j \neq 2^{k}-1\right] .
$$

Proof: The assertion that $h_{j}=0$ for $j+1=2^{k}$ is straightforward. Since the sequence

$$
\pi_{*} M O \rightarrow \pi_{*} H \mathbb{Z} / 2 \wedge M O \rightrightarrows \pi_{*} H \mathbb{Z} / 2 \wedge H \mathbb{Z} / 2 \wedge M O
$$

is a split equalizer, to show that the remaining $h_{j}$ are in $\pi_{*} M O$ it suffices to show that they are equalized by the parallel maps in (5.24). This works out to showing that the series (5.22) is invariant under substitutions of the form

$$
e \mapsto e+\sum \zeta_{m} e^{2^{m}}
$$

The series (5.22) is characterized as the unique isomorphism of the formal group law for unoriented cobordism with the additive group, having the additional property that the coefficients of $a^{2^{k}}$ are zero. This condition is stable under the substitutions (5.25). The last assertion follows from Quillen's characterization of $\pi_{*} M O$.

Remark 5.26. Recall the real orientation $\bar{x}$ of $i_{1}^{*} M U^{((G))}$ of Example 5.6. Applying the $R O(G)$-graded cohomology norm $(\S 2.3 .3)$ to $\bar{x}$, and then passing to geometric fixed points, gives a class

$$
\Phi^{G} N(\bar{x}) \in M O^{1}\left(\mathbf{R P}^{\infty}\right)
$$


One can easily check that $\Phi^{G} N(\bar{x})$ coincides with the $M O$ Euler class $a$ defined at the beginning of this section. Similarly one has

$$
\Phi^{G} N\left(\bar{x}_{H}\right)=e .
$$

Applying $\Phi^{G} N$ to $\log _{\bar{F}}$ and using the fact that it is a ring homomorphism (Proposition 2.59) gives

It follows that

$$
e=a+\sum \Phi^{G} N\left(\bar{m}_{k}\right) a^{k+1} .
$$

$$
\Phi^{G} N\left(\bar{m}_{k}\right)=\alpha_{k} .
$$

5.3. Refinement of homotopy groups. We begin by focusing on a simple consequence of Proposition 5.11.

Proposition 5.27. For every $m>1$, every element of

$$
\pi_{2 k}\left(\bigwedge^{m} M U\right)
$$

can be refined to an equivariant map

$$
S^{k \rho_{2}} \rightarrow \bigwedge^{m} M U_{\mathbb{R}} .
$$

This result expresses an important property of the $C_{2}$-spectra given by iterated smash products of $M U_{\mathbb{R}}$. Our goal in this section is to formulate a generalization to the case $G=C_{2^{n}}$.

Definition 5.28. Suppose $X$ is a $G$-spectrum. A refinement of $\pi_{k}^{u} X$ is a map

$$
c: \widehat{W} \rightarrow X
$$

in which $\widehat{W}$ is a wedge of slice cells of dimension $k$, inducing an isomorphism

$$
\pi_{k}^{u} \widehat{W} \rightarrow \pi_{k}^{u} X
$$

A refinement of the homotopy groups of $X$ (or a refinement of homotopy of $X$ ) is a map

$$
\widehat{W}=\bigvee \widehat{W}_{k} \rightarrow X
$$

whose restriction to each $\widehat{W}_{k}$ is a refinement of $\pi_{k}^{u}$.

Remark 5.29. Let $\sigma_{G}(\mathbb{Z})$ be the sign representation of $G$ on $\mathbb{Z}$. There is an $G$ module isomorphism $\pi_{|G|}^{u} S^{\rho_{G}} \approx \sigma_{G}(\mathbb{Z})$, and more generally

$$
\pi_{n|H|}^{u}\left(G_{+}{ }_{H}^{\wedge} S^{n \rho_{H}}\right) \approx \operatorname{ind}_{H}^{G} \sigma_{H}(\mathbb{Z})^{\otimes n} .
$$

This implies that when $k$ is even, a necessary condition for $\pi_{k}^{u} X$ to admit a refinement is that it be isomorphic as a $G$-module to a sum

$$
\bigoplus_{H \subset G} M_{H, k}
$$

where $M_{H, k}$ is zero unless $|H|$ divides $k$ and is a sum of copies of $\operatorname{ind}_{H}^{G}\left(\sigma_{H}(\mathbb{Z})^{\otimes \ell}\right)$ when $k=\ell|H|$. Adding the further condition that for every $H \subset G$, with $k=\ell|H|$, every element in $\pi_{k}^{u} X$ transforming in $\sigma_{H}(\mathbb{Z})^{\otimes \ell}$ refines to an element of $\pi_{\ell \rho_{H}}^{H} X$ makes it sufficient. A similar analysis describes the case in which $k$ is odd. 
Remark 5.30. Using Remark 5.29 one can check that a refinement of $\pi_{k}^{u} X$ consists of isotropic slice cells if and only if $\pi_{k}^{u} X$ does not contain a free $G$-module as a summand.

The splitting (5.12) used to prove Proposition 5.27 is multiplicative. This too has an important analogue.

Definition 5.31. Suppose that $R$ is an equivariant associative algebra. A multiplicative refinement of homotopy is an associative algebra map $\widehat{W} \rightarrow R$ which, when regarded as a map of $G$-spectra is a refinement of homotopy.

Proposition 5.32. For every $m \geq 1$ there exists a multiplicative refinement of homotopy

$$
\widehat{W} \rightarrow \bigwedge^{m} M U^{((G))},
$$

with $\widehat{W}$ a wedge of regular isotropic slice cells.

Two ingredients form the proof of Proposition 5.32. The first, Lemma 5.33 below, is a description of $\pi_{*}^{u} M U^{((G))}$ as a $G$-module. The computation is of interest in its own right, and is used elsewhere in this paper. It is proved in $\$ 5.4$. The second is the classical description of $\pi_{*}^{u}\left(\bigwedge^{m} M U^{((G))}\right), m>1$, as a $\pi_{*}^{u} M U^{((G))}$-module.

Lemma 5.33. There is a sequence of elements $r_{i} \in \pi_{2 i}^{u} M U^{((G))}$ with the property that

$$
\pi_{*}^{u} M U^{((G))}=\mathbb{Z}_{2}\left[G \cdot r_{1}, G \cdot r_{2}, \ldots\right],
$$

in which $G \cdot r_{i}$ stands for the sequence

$$
\left(r_{i}, \ldots \gamma^{\frac{g}{2}-1} r_{i}\right)
$$

of length $g / 2$.

We refer to the condition (5.34) by saying that the elements $r_{i} \in \pi_{2 i}^{u} M U^{((G))}$ form a set of $G$-algebra generators for $\pi_{*}^{u} M U^{((G))}$.

Remark 5.35. Lemma 5.33 completely describes $\pi_{*}^{u} M U^{((G))}$ as a representation of $G$. To spell it out, recall from Remark 5.14 that the action of the generator of $C_{2}$ on $\pi_{2 i}^{u} M U^{((G))}$ is by $(-1)^{i}$. The elements $r_{i} \in \pi_{2 i}^{u} M U^{((G))}$ therefore satisfy $\gamma^{\frac{g}{2}} r_{i}=$ $(-1)^{i} r_{i}$ and transform in the representation induced from the sign representation of $C_{2}$ if $i$ is odd and in the representation induced from the trivial representation of $C_{2}$ if $i$ is even. Lemma 5.33 implies that the map from the symmetric algebras on the sum of these representations to $\pi_{*}^{u} M U^{((G))}$ is an isomorphism.

Remark 5.36. The fact that the action of $C_{2}$ on $\pi_{2 i}^{u} M U^{((G))}$ is either a sum of sign or trivial representations means that it cannot contain a summand which is free. The same is therefore true of the $G$-action. By Remark 5.30 this implies that only isotropic slice cells may occur in a refinement of $\pi_{2 i}^{u} M U^{((G))}$.

Over $\pi_{*}^{u} M U^{((G))} \wedge M U^{((G))}$, there are two formal group laws, $F_{L}$ and $F_{R}$ coming from the canonical orientations of the left and right factors. There is also a canonical isomorphism between them, which can be written as

$$
x_{R}=\sum b_{j} x_{L}^{j+1} \text {. }
$$

Write

$$
G \cdot b_{i}
$$


for the sequence

$$
b_{i}, \gamma b_{i}, \ldots, \gamma^{g / 2-1} b_{i} .
$$

The following result is a standard computation in complex cobordism.

Lemma 5.37. The ring $\pi_{*}^{u} M U^{((G))} \wedge M U^{((G))}$ is given by

$$
\pi_{*}^{u} M U^{((G))} \wedge M U^{((G))}=\pi_{*}^{u} M U^{((G))}\left[G \cdot b_{1}, G \cdot b_{2}, \ldots\right] .
$$

For $m>1$,

$$
\pi_{*}^{u} \bigwedge^{m} M U^{((G))}=\pi_{*}^{u} M U^{((G))} \wedge \bigwedge^{m-1} M U^{((G))}
$$

is the polynomial ring

$$
\pi_{*}^{u} M U^{((G))}\left[G \cdot b_{i}^{(j)}\right]
$$

with

$$
\begin{aligned}
& i=1,2, \ldots, \quad \text { and } \\
& j=1, \ldots, m-1 .
\end{aligned}
$$

The element $b_{i}^{(j)}$ is the class $b_{i}$ arising from the $j^{\text {th }}$ factor of $M U^{((G))}$ in $\bigwedge^{m-1} M U^{((G))}$.

Proof: The second assertion follows from the first and the Künneth formula. If not for the fact that $G$ acts on both factors of $i_{0}^{*} M U^{((G))}$, the first assertion would also follow immediately from the Künneth formula and the usual description of $M U_{*} M U$. The quickest way to deduce it from the apparatus we have describe so far is to let $G \subset G^{\prime}$ be an embedding of index 2 into a cyclic group, write

$$
M U^{((G))} \wedge M U^{((G))} \approx i_{G}^{*} M U^{\left(\left(G^{\prime}\right)\right)}
$$

and use Corollary 5.20.

Remark 5.38. As with Lemma 5.33, the lemma above actually determines the structure of $\pi_{*}^{u} M U^{((G))} \wedge M U^{((G))}$ as a $G$-equivariant $\pi_{*}^{u} M U^{((G))}$-algebra. See Remark 5.35.

Proof of Proposition 5.32, assuming Lemma 5.33: This is a straightforward application of the method of twisted monoid rings of $\$ 2.4$. To keep the notation simple we begin with the case $m=1$. Choose a sequence $r_{i} \in \pi_{2 i}^{u} M U^{((G))}$ with the property described in Lemma 5.33. Let

$$
\bar{r}_{i}: S^{i \rho_{2}} \rightarrow i_{1}^{*} M U^{((G))},
$$

be a representative of the image of $r_{i}$ under the splitting (5.12). Since $M U^{((G))}$ is a commutative algebra, the method of twisted monoid rings can be used to construct an associative algebra map

$$
S^{0}\left[G \cdot \bar{r}_{1}, G \cdot \bar{r}_{2}, \ldots\right] \rightarrow M U^{((G))},
$$

Using Proposition 4.7 one can easily check that $S^{0}\left[G \cdot \bar{r}_{1}, G \cdot \bar{r}_{2}, \ldots\right]$ is a wedge of regular isotropic $G$-slice cells. Using Lemma 5.33 one then easily checks that (5.40) is multiplicative refinement of homotopy. The case $m \geq 1$ is similar, using in addition Lemma 5.37 and the collection $\left\{r_{i}, b_{i}(j)\right\}$. 
5.4. Algebra generators for $\pi_{*}^{u} M U^{((G))}$. In this section we will describe convenient algebra generators for $\pi_{*}^{u} M U^{((G))}$. Our main results are Proposition 5.45 (giving a criterion for a sequence of elements $r_{i}$ to "generate" $\pi_{*}^{u} M U^{((G))}$ as a $G$ algebra, as in Lemma 5.33) and Corollary 5.49 (specifying a particular sequence of $\left.r_{i}\right)$. Proposition 5.45 directly gives Lemma 5.33 .

We remind the reader that the notation $H_{*}^{u} X$ refers to the homology groups $H_{*}\left(i_{0}^{*} X\right)$ of the non-equivariant spectrum underlying $X$.

5.4.1. A criterion for a generating set. Let

$$
m_{i} \in H_{2 i} M U=\pi_{2 i}^{u} H \underline{\mathbb{Z}} \wedge M U_{\mathbb{R}}
$$

be the coefficient of the universal logarithm. Using the identification (5.2)

$$
i_{1}^{*} M U^{((G))}=\bigwedge_{j=0}^{g / 2-1} \gamma^{j} M U_{\mathbb{R}}
$$

and the Künneth formula, one has

$$
H_{*}^{u} M U^{((G))}=\mathbb{Z}_{(2)}\left[\gamma^{j} m_{k}\right]
$$

where

$$
\begin{gathered}
k=1,2, \ldots, \\
j=0, \ldots, g / 2-1 .
\end{gathered}
$$

By the definition of the $\gamma^{j} m_{k}$ and Remark 5.14, the action of $G$ on $H_{*}^{u} M U^{((G))}$ is given by

$$
\gamma \cdot \gamma^{j} m_{k}= \begin{cases}\gamma^{j+1} m_{k} & j<g / 2-1 \\ (-1)^{k} m_{k} & j=g / 2-1\end{cases}
$$

Let

$$
\begin{gathered}
I=\operatorname{ker} \pi_{*}^{u} M U^{((G))} \rightarrow \mathbb{Z}_{(2)} \\
I_{H}=\operatorname{ker} H_{*}^{u} M U^{((G))} \rightarrow \mathbb{Z}_{(2)}
\end{gathered}
$$

denote the augmentation ideals, and

$$
\begin{gathered}
Q_{*}=I / I^{2} \\
Q H_{*}=I_{H} / I_{H}^{2}
\end{gathered}
$$

the modules of indecomposable, with $Q_{2 m}$ and $Q H_{2 m}$ indicating the homogeneous parts of degree $2 m$ (the odd degree parts are zero). The module $Q H_{*}$ is the free abelian group with basis $\left\{\gamma^{j} m_{k}\right\}$, and from Milnor [65], one knows that the Hurewicz homomorphism gives an isomorphism

$$
Q_{2 k} \rightarrow Q H_{2 k}
$$

if $2 k$ is not of the form $2\left(2^{\ell}-1\right)$, and an exact sequence

$$
Q_{2\left(2^{\ell}-1\right)} \longmapsto Q H_{2\left(2^{\ell}-1\right)} \rightarrow \mathbb{Z} / 2
$$

in which the rightmost map is the one sending each $\gamma^{j} m_{k}$ to 1 .

Formula (5.41) implies that the $G$-module $Q H_{2 k}$ is the module induced from the sign representation of $C_{2}$ if $k$ is odd and from the trivial representation if $k$ is even. 
Lemma 5.43. Let $r=\sum a_{j} \gamma^{j} m_{k} \in Q H_{2 k}$. The unique G-module map

$$
\begin{aligned}
\mathbb{Z}_{(2)}[G] & \rightarrow Q H_{2 k} \\
1 & \mapsto r
\end{aligned}
$$

factors through a map

$$
\mathbb{Z}_{(2)}[G] /\left(\gamma^{g / 2}-(-1)^{k}\right) \rightarrow Q H_{2 k}
$$

which is an isomorphism if and only if $\sum a_{j} \equiv 1 \bmod 2$.

Proof: The factorization is clear, since $\gamma^{g / 2}$ acts with eigenvalue $(-1)^{k}$ on $Q H_{2 k}$. Use the unique map $\mathbb{Z}_{(2)}[G] \rightarrow Q H_{2 k}$ sending 1 to $m_{k}$ to identify $Q H_{2 k}$ with $A=$ $\mathbb{Z}_{(2)}[G] /\left(\gamma^{g / 2}-(-1)^{k}\right)$. The main assertion is then that an element $r=\sum a_{j} \gamma^{j} \in A$ is a unit if and only if $\sum a_{j} \equiv 1 \bmod 2$. Since $A$ is a finitely generated free module over the Noetherian local ring $\mathbb{Z}_{(2)}$, Nakayama's lemma implies that the map $A \rightarrow A$ given by multiplication by $r$ is an isomorphism if and only if it is after reduction modulo 2. So $r$ is a unit if and only if it is after reduction modulo 2. But $A /(2)=\mathbb{Z} / 2[\gamma] /\left(\gamma^{g / 2}-1\right)$ is a local ring with nilpotent maximal ideal $(\gamma-1)$. The residue map

$$
A /(2) \rightarrow A /(2, \gamma-1)=\mathbb{Z} / 2
$$

sends $\sum a_{j} \gamma^{j} m_{k}$ to $\sum a_{j}$. The result follows.

Lemma 5.44. The $G$-module $Q_{2\left(2^{\ell}-1\right)}$ is isomorphic to the module induced from the sign representation of $C_{2}$. For $y \in Q_{2\left(2^{\ell}-1\right)}$, the unique $G$-map

$$
\begin{aligned}
\mathbb{Z}_{(2)}[G] & \rightarrow Q H_{2\left(2^{\ell}-1\right)} \\
1 & \mapsto y
\end{aligned}
$$

factors through a map

$$
A=\mathbb{Z}_{(2)}[G] /\left(\gamma^{g / 2}+1\right) \rightarrow Q_{2\left(2^{\ell}-1\right)}
$$

which is an isomorphism if and only if $y=(1-\gamma) r$ where $r \in Q H_{2\left(2^{\ell}-1\right)}$ satisfies the condition $\sum a_{j}=1 \bmod 2$ of Lemma 5.43.

Proof: Identify $Q H_{2\left(2^{\ell}-1\right)}$ with $A$ by the map sending 1 to $m_{2^{\ell}-1}$. In this case $A$ is isomorphic to $\mathbb{Z}_{(2)}[\zeta]$, with $\zeta$ a primitive $g^{\text {th }}$ root of unity, and in particular is an integral domain. Under this identification, the rightmost map in (5.42) is the quotient of $A$ by the principal ideal $(\zeta-1)$. Since $A$ is an integral domain, this ideal is a rank 1 free module generated by any element of the form $(1-\gamma) r$ with $r \in A$ a unit. The result follows.

This discussion proves

Proposition 5.45. Let

$$
\left\{r_{1}, r_{2}, \ldots\right\} \subset \pi_{*}^{u} M U^{((G))}
$$

be any sequence of elements whose images

$$
s_{k} \in Q H_{2 k}
$$

have the property that for $k \neq 2^{\ell}-1, s_{k}=\sum a_{j} \gamma^{j} m_{k}$ with

$$
\sum a_{j} \equiv 1 \bmod 2
$$


and $s_{2^{\ell}-1}=(1-\gamma)\left(\sum a_{j} \gamma^{j} m_{2^{\ell}-1}\right)$, with

$$
\sum a_{j} \equiv 1 \quad \bmod 2 \text {. }
$$

Then the sequence

$$
\left\{r_{1}, \ldots \gamma^{\frac{g}{2}-1} r_{1}, r_{2}, \ldots, \gamma^{\frac{g}{2}-1} r_{2}, \ldots\right\}
$$

generates the ideal $I$, and so

$$
\mathbb{Z}_{(2)}\left[r_{1}, \ldots \gamma^{\frac{g}{2}-1} r_{1}, r_{2}, \ldots, \gamma^{\frac{g}{2}-1} r_{2}, \ldots\right] \rightarrow \pi_{*}^{u} M U^{((G))}
$$

is an isomorphism.

5.4.2. Specific generators. We now use the action of $G$ on $i_{1}^{*} M U^{((G))}$ to define specific elements $\bar{r}_{i} \in \pi_{i \rho_{2}}^{C_{2}} M U^{((G))}$ refining a sequence satisfying the condition of Proposition 5.45.

Write

$$
\bar{F}(\bar{x}, \bar{y})
$$

for the formal group law over $\pi_{\star}^{C_{2}} M U^{((G))}$, and

$$
\log _{\bar{F}}(\bar{x})=\bar{x}+\sum_{i>0} \bar{m}_{k} \bar{x}^{k+1}
$$

for its logarithm. This defines elements

$$
\bar{m}_{k} \in \pi_{k \rho_{2}}^{C_{2}} H \underline{\mathbb{Z}}_{(2)} \wedge M U^{((G))} .
$$

We define the elements

$$
\bar{r}_{k} \in \pi_{k \rho_{2}}^{C_{2}} M U^{((G))}
$$

to be the coefficients of the unique strict isomorphism of $\bar{F}$ with the 2-typification of $\bar{F}^{\gamma}$. The Hurewicz images

$$
\bar{r}_{k} \in \pi_{k \rho_{2}}^{C_{2}} H \underline{\mathbb{Z}}_{(2)} \wedge M U^{((G))}
$$

are given by the power series identity

$$
\sum \bar{r}_{k} \bar{x}^{k+1}=\left(\bar{x}+\sum \gamma\left(\bar{m}_{2^{\ell}-1}\right) \bar{x}^{2^{\ell}}\right)^{-1} \circ \log _{\bar{F}}(\bar{x}) .
$$

Modulo decomposables this becomes

$$
\bar{r}_{k} \equiv \begin{cases}\bar{m}_{k}-\gamma \bar{m}_{k} & k=2^{\ell}-1 \\ \bar{m}_{k} & \text { otherwise. }\end{cases}
$$

This shows that the elements $\bar{r}_{k}$ satisfy the condition of Proposition 5.45, hence

Corollary 5.49. The classes $r_{k}=i_{0}^{*} \bar{r}_{k}$ form a set of G-algebra generators for $\pi_{*}^{u} M U^{((G))}$.

These are the specific generators with which we shall work. Though it does not appear in the notation, the classes $\bar{r}_{i}$ depend on the group $G$. In $\S 9$ we will need to consider the classes $\bar{r}_{i}$ for a group $G$ and for a subgroup $H \subset G$. We will then use the notation

to distinguish them.

$$
\bar{r}_{i}^{H} \text { and } \bar{r}_{i}^{G}
$$

The following result establishes an important property of these specific $\bar{r}_{k}$. In the statement below, the symbol $N$ is the norm map on the $R O(G)$-graded homotopy groups of commutative rings. 
Proposition 5.50. For all $k$

$$
\Phi^{G} N\left(\bar{r}_{k}\right)=h_{k} \in \pi_{k} M O,
$$

where the $h_{k}$ are the classes defined in §5.2.2. In particular, the set

$$
\left\{\Phi^{G} N\left(\overline{r_{k}}\right) \mid k \neq 2^{\ell}-1\right\}
$$

is a set of polynomial algebra generators of $\pi_{*} M O$, and for all $\ell$

$$
\Phi^{G} N\left(\bar{r}_{2^{\ell}-1}\right)=h_{2^{\ell}-1}=0 .
$$

Proof: From Remark 5.26 we know that

$$
\begin{aligned}
\Phi^{G} N \bar{x} & =a \\
\Phi^{G} N \bar{x}_{H} & =e \\
\Phi^{G} N \bar{m}_{n} & =\alpha_{n} .
\end{aligned}
$$

Corollary 2.29 implies that

$$
\Phi^{G} N \gamma \bar{m}_{n}=\Phi^{G} N \bar{m}_{n}
$$

so we also know that

$$
\Phi^{G} N \gamma \bar{m}_{n}=\alpha_{n} .
$$

Since the Hurewicz homomorphism

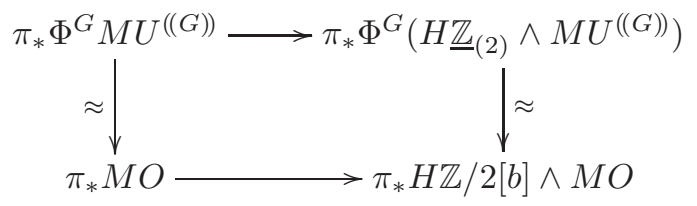

is a monomorphism, we can calculate $\Phi^{G} N \bar{r}_{k}$ using (5.47). Applying $\Phi^{G} N$ to (5.47), and using the fact that it is a ring homomorphism gives

$$
\begin{aligned}
a+ & \sum\left(\Phi^{G} N \bar{r}_{k}\right) a^{k+1} \\
& =\left(a+\sum\left(\Phi^{G} N \gamma \bar{m}_{2^{\ell-1}}\right) a^{2^{\ell}}\right)^{-1} \circ\left(a+\sum\left(\Phi^{G} N \bar{m}_{k}\right) a^{k+1}\right) \\
& =\left(a+\sum \alpha_{2^{\ell-1}} a^{2^{\ell}}\right)^{-1} \circ\left(a+\sum \alpha_{k} a^{k+1}\right) .
\end{aligned}
$$

But this is the identity defining the classes $h_{k}$.

In addition to

$$
h_{k}=\Phi^{G} N\left(\bar{r}_{k}\right) \in \pi_{k} \Phi^{G} M U^{((G))}=\pi_{k} M O
$$

there are some important classes $f_{k}$ attached to these specific $\bar{r}_{k}$.

Definition 5.51. Set

$$
f_{k}=a_{\bar{\rho}_{G}}^{k} N \bar{r}_{k} \in \pi_{k}^{G} M U^{((G))},
$$

where $\bar{\rho}_{G}=\rho_{G}-1$ is the reduced regular representation. 
The relationship between these classes is displayed in the following commutative diagram.

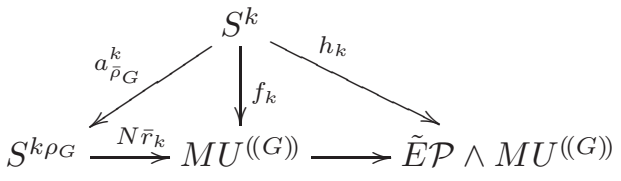

\section{The Slice Theorem and the Reduction Theorem}

Using the method of twisted monoid rings one can show the Slice Theorem and the Reduction Theorem to be equivalent. In $\S 6.1$ we formally state the Reduction Theorem, and assuming it, prove the Slice Theorem. In $\S 6.2$ we establish a converse, for associative algebras $R$ which are pure and which admit a multiplicative refinement of homotopy by a polynomial algebra. Both assertions are used in the proof of the Reduction Theorem in $\S 7$.

6.1. From the Reduction Theorem to the Slice Theorem. We now state the Slice Theorem, using the language of $\S 4.6 .2$.

Theorem 6.1 (Slice Theorem). The spectrum $M U^{((G))}$ is an isotropic pure spectrum.

For the proof of the slice theorem, let

$$
A=S^{0}\left[G \cdot \bar{r}_{1}, G \cdot \bar{r}_{2}, \ldots\right] \rightarrow M U^{((G))}
$$

be the multiplicative refinement of homotopy constructed in $\$ 5.3$ using the method of twisted monoid rings, and the specific generators of $\$ 5.4 .2$. Let $J$ be the left $G$-set defined by

$$
J=\coprod_{i} G / C_{2} .
$$

As described in $\S 2.4$, the spectrum $A$ is the indexed wedge

$$
A=\bigvee_{f \in \mathbb{N}_{0}^{J}} S^{\rho_{f}},
$$

in which $\rho_{f}$ is the unique multiple of the regular representation of the stabilizer group of $f$ having dimension

$$
\operatorname{dim} f=2 \sum_{j \in J} j f(j) .
$$

As in Example 2.33, let

$$
M_{d} \subset A
$$

be the monomial ideal consisting of the indexed wedge of the $S^{\rho_{f}}$ with $\operatorname{dim} f \geq d$. Then $M_{2 d-1}=M_{2 d}$, and the $M_{2 d}$ fit into a sequence

$$
\cdots \hookrightarrow M_{2 d+2} \hookrightarrow M_{2 d} \hookrightarrow M_{2 d-2} \hookrightarrow \cdots .
$$

The quotient

is the indexed wedge

$$
M_{2 d} / M_{2 d+2}
$$

$$
\widehat{W}_{2 d}=\bigvee_{\operatorname{dim} f=2 d} S^{\rho_{f}}
$$


on which $A$ is acting through the multiplicative map $A \rightarrow S^{0}$ (Examples 2.33 and A.49). The $G$-spectrum (6.2) is a wedge of regular isotropic slice cells of dimension $2 d$.

Replace $M U^{((G))}$ with a cofibrant $A$-module, and form

$$
K_{2 d}=M U^{((G))} \hat{A}_{\wedge} M_{2 d}
$$

The $K_{2 d}$ fit into a sequence

$$
K_{2 d+2} \hookrightarrow K_{2 d} \hookrightarrow \cdots .
$$

Lemma 6.3. The sequences

$$
\begin{gathered}
K_{2 d+2} \rightarrow K_{2 d} \rightarrow K_{2 d} / K_{2 d+2} \\
K_{2 d} / K_{2 d+2} \rightarrow M U^{((G))} / K_{2 d+2} \rightarrow M U^{((G))} / K_{2 d}
\end{gathered}
$$

are weakly equivalent to cofibration sequences. There is an equivalence

$$
K_{2 d} / K_{2 d+2} \approx R(\infty) \wedge \widehat{W}_{2 d}
$$

in which

$$
R(\infty)=M U^{((G))}{ }_{A}^{\wedge} S^{0}
$$

Proof: Since the map $K_{2 d+2} \rightarrow K_{2 d}$ is the inclusion of a wedge summand it is an $h$-cofibration of spectra, and the first assertion follows from Proposition B.20 and Corollary B.140. The second assertion follows from the associativity of the smash product

$$
M U^{((G))}{ }_{A}\left(M_{2 d} / M_{2 d+1}\right) \approx\left(M U^{((G))}{\underset{A}{\wedge}}_{S} S^{0}\right) \wedge \widehat{W}_{2 d} \approx R(\infty) \wedge \widehat{W}_{2 d} .
$$

This completes the proof.

The Thom map

$$
M U^{((G))} \rightarrow H \underline{\mathbb{Z}}_{(2)}
$$

factors uniquely through an $M U^{((G))}$-module map

$$
R(\infty) \rightarrow H \underline{\mathbb{Z}}_{(2)} .
$$

The following important result will be proved in $\S 7.3$.

Theorem 6.5 (The Reduction Theorem). The map

$$
R(\infty) \rightarrow H \underline{\mathbb{Z}}_{(2)}
$$

is a weak equivalence.

The case $G=C_{2}$ of the Reduction Theorem is Proposition 4.9 of Hu-Kriz[35]. Its analogue in motivic homotopy theory appears in unpublished work of the second author and Morel.

To deduce the Slice Theorem from Theorem 6.5 we need two simple lemmas.

Lemma 6.6. The spectrum $K_{2 d+2}$ is slice $2 d$-positive. 
Proof: The class of left $A$-modules $M$ for which $M \underset{A}{\wedge} M_{2 d+2}>2 d$ is closed under homotopy colimits and extensions. It contains every module of the form $\Sigma^{k} G / H_{+} \wedge A$, with $k \geq 0$. Since $A$ is $(-1)$-connected this means it contains every $(-1)$-connected cofibrant $A$-module. In particular it contains the cofibrant replacement of $M U^{((G))}$.

Lemma 6.7. If Theorem 6.5 holds then $M U^{((G))} / K_{2 d+2} \leq 2 d$.

Proof: This is easily proved by induction on $d$, using the fact that

$$
R(\infty) \wedge \widehat{W}_{2 d} \rightarrow M U^{((G))} / K_{2 d+2} \rightarrow M U^{((G))} / K_{2 d} .
$$

is weakly equivalent to a cofibration sequence (Lemma 6.3).

Proof of the Slice Theorem assuming the Reduction Theorem: It follows from the fibration sequence

$$
K_{2 d+2} \rightarrow M U^{((G))} \rightarrow M U^{((G))} / K_{2 d+2},
$$

Lemmas 6.6 and 6.7 above, and Lemma 4.16 that

$$
P^{2 d+1} M U^{((G))} \approx P^{2 d} M U^{((G))} \approx M U^{((G))} / K_{2 d+2} .
$$

Thus the odd slices of $M U^{((G))}$ are contractible and the $2 d$-slice is weakly equivalent to

$$
R(\infty) \wedge \widehat{W}_{2 d} \approx H \underline{\mathbb{Z}}_{(2)} \wedge \widehat{W}_{2 d}
$$

This completes the proof.

6.2. A converse. The arguments of the previous section can be reversed. Suppose that $R$ is a $(-1)$-connected associative algebra which we know in advance to be pure, and that $A \rightarrow R$ is a multiplicative refinement of homotopy, with

$$
A=S^{0}\left[G \cdot \bar{x}_{1}, \ldots\right]
$$

a twisted monoid ring having the property that $\left|\bar{x}_{i}\right|>0$ for all $i$. Note that this implies that $\pi_{0}^{u} R=\mathbb{Z}$ and that $P_{0}^{0} R=H \underline{\mathbb{Z}}$. Let $M_{d+1} \subset A$ be the monomial ideal consisting of the slice cells in $A$ of dimension $>d$, write

$$
\tilde{P}_{d+1} R=M_{d+1} \stackrel{\wedge}{A} R
$$

and

$$
\tilde{P}^{d} R=R / \tilde{P}_{d+1} R \approx\left(A / M_{d+1}\right) \bigwedge_{A} R
$$

Then the $\tilde{P}^{d} R$ form a tower. Since $M_{d+1}>d$ and $R \geq 0$ (Proposition 4.20), the spectrum $\tilde{P}_{d+1} R$ is slice $d$-positive. There is therefore a map

$$
\tilde{P}^{d} R \rightarrow P^{d} R
$$

compatible with variation in $d$.

Proposition 6.9. The map (6.8) is a weak equivalence. The tower $\left\{\tilde{P}^{d} R\right\}$ is the slice tower for $R$. 
By analogy with the slice tower, write $\tilde{P}_{d^{\prime}}^{d} R$ for the homotopy fiber of the map

$$
\tilde{P}^{d} R \rightarrow \tilde{P}^{d^{\prime}-1} R
$$

when $d^{\prime} \leq d$.

We start with a lemma concerning the case $d=0$.

Lemma 6.10. Let $n \geq 0$. If the map

$$
\tilde{P}^{0} R \rightarrow P^{0} R
$$

becomes an equivalence after applying $P^{n}$, then for every $d \geq 0$ the map

$$
\tilde{P}_{d}^{d} R \rightarrow P_{d}^{d} R
$$

becomes an equivalence after applying $P^{d+n}$.

Proof: Write $\widehat{W}_{d}=M_{d} / M_{d+1}$. Then there are equivalences

$$
\tilde{P}_{d}^{d} R \approx \widehat{W}_{d} \widehat{A}_{A} R \approx \widehat{W}_{d} \wedge\left(S^{0} \widehat{A}_{A} R\right) \approx \widehat{W}_{d} \wedge \tilde{P}_{0}^{0} R
$$

Since $A \rightarrow R$ is a refinement of homotopy and $R$ is pure, the analogous map

$$
\widehat{W}_{d} \wedge P_{0}^{0} R \rightarrow P_{d}^{d} R
$$

is also a weak equivalence. Now consider the following diagram

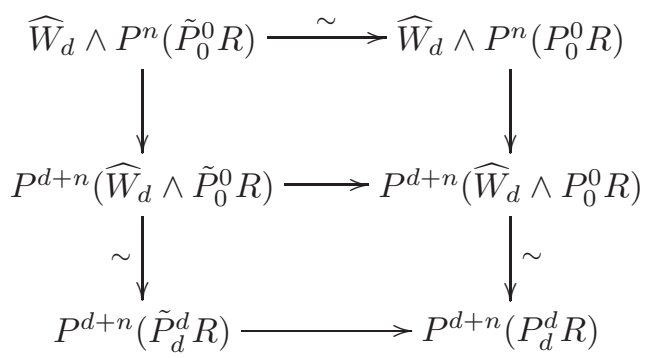

The top map is an equivalence by assumption. The bottom vertical maps are the result of applying $P^{d+n}$ to the weak equivalences just described. Since $\widehat{W}_{d}$ is a wedge of regular slice cells of dimension $d$, Corollary 4.25 implies that the upper vertical maps are weak equivalences. It follows that the bottom horizontal map is a weak equivalence as well.

Proof of Proposition 6.9: We will show by induction on $k$ that for all $d$, the map

$$
P^{d+k}\left(\tilde{P}^{d} R\right) \rightarrow P^{d+k}\left(P^{d} R\right)
$$

is a weak equivalence. By the strong convergence of the slice tower (Theorem 4.42) this will give the result. The induction starts with $k=0$ since $\tilde{P}_{d+1} R>d$ and so $R \rightarrow \tilde{P}^{d} R$ is a $P^{d}$-equivalence. For the induction step, suppose we know the result for some $k>0$, and consider

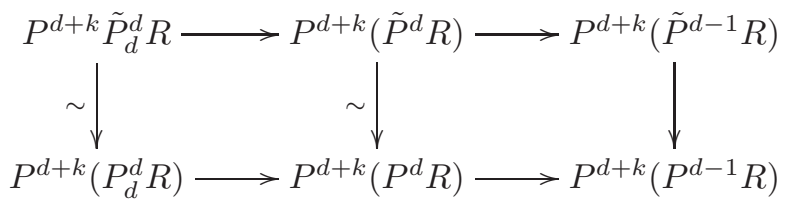


The bottom row is a cofibration sequence since it can be identified with

$$
P_{d}^{d} R \rightarrow P^{d} R \rightarrow P^{d-1} R .
$$

The middle vertical map is a weak equivalence by the induction hypothesis, and the left vertical map is a weak equivalence by the induction hypothesis and Lemma 6.10. It follows that the cofiber of the upper left map is weakly equivalent to $P^{d+k}\left(P^{d-1} R\right)$ and hence is $(d+k+1)$-slice null (in fact $d$ slice null). The top row is therefore a cofibration sequence by Corollary 4.17, and so the rightmost vertical map is a weak equivalence. This completes the inductive step, and the proof.

\section{The Reduction Theorem}

We will prove the Reduction Theorem by induction on $g=|G|$. The case in which $G$ is the trivial group follows from Quillen's results. We may therefore assume that we are working with a non-trivial group $G$ and that the Reduction Theorem is known for all proper subgroups of $G$. In the first subsection below we collect some consequences of this induction hypothesis. The proof of the induction step is in $\S 7.3$.

7.1. Consequences of the induction hypothesis. This next result holds for general $G$.

Lemma 7.1. Suppose that $X$ is pure spectrum and $\widehat{W}$ is a wedge of regular slice cells. Then $\widehat{W} \wedge X$ is pure. If $X$ is pure and isotropic and $\widehat{W}$ is regular isotropic, then $\widehat{W} \wedge X$ is pure and isotropic.

Proof: Using Proposition 4.21 one reduces to the case in which $\widehat{W}=S^{m \rho_{G}}$. In that case the claim follows from Corollary 4.25.

Proposition 7.2. Suppose $H \subset G$ has index 2. If the Slice Theorem holds for $H$ then the spectrum $i_{H}^{*} M U^{((G))}$ is an isotropic pure spectrum.

Proof: This is an easy consequence of Corollary 5.20, which gives an associative algebra equivalence

$$
i_{H}^{*} M U^{((G))} \approx M U^{((H))}\left[H \cdot \bar{b}_{1}, H \cdot \bar{b}_{2}, \ldots\right] .
$$

This shows that $i_{H}^{*} M U^{((G))}$ is a wedge of smash products of even dimensional isotropic slice cells with $M U^{((H))}$, and hence (by Lemma 7.1) an isotropic pure spectrum since $M U^{((H))}$ is.

Proposition 7.3. Suppose $H \subset G$ has index 2. If the Slice Theorem holds for $H$ then the map

$$
i_{H}^{*} R(\infty) \rightarrow i_{H}^{*} H \mathbb{Z}_{(2)}
$$

is an equivalence.

Proof: By Proposition 7.2 we know that $i_{H}^{*} M U^{((G))}$ is pure. The claim then follows from Proposition 6.9. 
7.2. Certain auxiliary spectra. Our proof of the Reduction Theorem will require certain auxiliary spectra. For an integer $k>0$ we define

$$
R(k)=M U^{((G))} /\left(G \cdot \bar{r}_{1}, \ldots, G \cdot \bar{r}_{k}\right)=M U^{((G))} \hat{A}_{A} A^{\prime}
$$

where

$$
\begin{aligned}
A & =S^{0}\left[G \cdot \bar{r}_{1}, G \cdot \bar{r}_{2}, \ldots\right] \\
A^{\prime} & =S^{0}\left[G \cdot \bar{r}_{k+1}, G \cdot \bar{r}_{k+2}, \ldots\right] .
\end{aligned}
$$

The spectrum $R(k)$ is a right $A^{\prime}$-module, and as in the case of $M U^{((G))}$ described in $\S 6$, the filtration of $A^{\prime}$ by the "dimension" monomial ideals leads to a filtration of $R(k)$ whose associated graded spectrum is

$$
R(\infty) \wedge A^{\prime} .
$$

Thus the reduction theorem also implies that $R(k)$ is a pure isotropic spectrum. By the results of the previous section, the induction hypothesis implies that $i_{H}^{*} R(k)$ is pure and isotropic.

We know from Proposition 4.64 that when $m$ is even, the slice $P_{m}^{m} R(k)$ is given by

$$
P_{m}^{m} R(k) \approx H \mathbb{Z}_{(2)} \wedge \widehat{W}_{m}
$$

where $\widehat{W} \subset A^{\prime}$ is the summand consisting of the wedge of slice cells of dimension $m$. When $m$ is odd the above discussion implies that $T \wedge P_{m}^{m} R(k)$ is contractible for any $G$-CW spectrum $T$ built entirely from induced $G$-cells. In particular, the equivariant homotopy groups of $E \mathcal{P}_{+} \wedge R(k)$ may be investigated by smashing the slice tower of $R(k)$ with $E \mathcal{P}_{+}$, and we will do so in $\S 7.3$, where we will exploit some very elementary aspects of the situation.

7.3. Proof of the Reduction Theorem. As mentioned at the beginning of the section, our proof of the Reduction Theorem is by induction on $|G|$, the case of the trivial group being a result of Quillen. We may therefore assume that $G$ is non-trivial, and that the result is known for all proper subgroups $H \subset G$. By Proposition 7.3 this implies that the map

$$
R(\infty) \rightarrow H \underline{\mathbb{Z}}_{(2)}
$$

becomes a weak equivalence after applying $i_{H}^{*}$.

For the induction step we smash the map in question with the isotropy separation sequence (2.44)

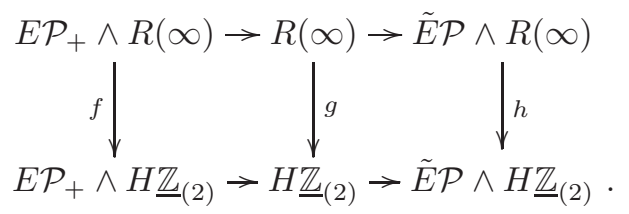

By the induction hypothesis, the map $f$ is an equivalence. It therefore suffices to show that the map $h$ is, and that, as discussed in Remark 2.49, is equivalent to showing that

$$
\pi_{*}^{G} h: \pi_{*} \Phi^{G} R(\infty) \rightarrow \pi_{*} \Phi^{G} H \underline{\mathbb{Z}}_{(2)}
$$

is an isomorphism.

We first show that the two groups are abstractly isomorphic. 
Proposition 7.5. The ring $\pi_{*} \Phi^{G} H \underline{\mathbb{Z}}_{(2)}$ is given by

$$
\pi_{*} \Phi^{G} H \underline{\mathbb{Z}}_{(2)}=\mathbb{Z} / 2[b]
$$

with

$$
b=u_{2 \sigma} a_{\sigma}^{-2} \in \pi_{2} \Phi^{G} H \underline{\mathbb{Z}}_{(2)} \subset a_{\sigma}^{-1} \pi_{\star}^{G} H \underline{\mathbb{Z}}_{(2)} .
$$

The groups $\pi_{*} \Phi^{G} R(\infty)$ are given by

$$
\pi_{n} \Phi^{G} R(\infty)= \begin{cases}\mathbb{Z} / 2 & n \geq 0 \text { even } \\ 0 & \text { otherwise }\end{cases}
$$

Proof: The first assertion is a restatement of Proposition 3.18. For the second we will make use of the monoidal geometric fixed point functor $\Phi_{M}^{G}$. The main technical issue is to take care that at key points in the argument we are working with spectra $X$ for which $\Phi^{G} X$ and $\Phi_{M}^{G} X$ are weakly equivalent.

Recall the definition

$$
R(\infty)=M U_{c}^{((G))}{ }_{A}^{\wedge} S^{0},
$$

where for emphasis we've written $M U_{c}^{((G))}$ as a reminder that $M U^{((G))}$ has been replaced by a cofibrant $A$-module (see $\$ 2.4$ ). Proposition B.208 implies that $R(\infty)$ is cofibrant, so there is an isomorphism

$$
\pi_{*} \Phi^{G} R(\infty) \approx \pi_{*} \Phi_{M}^{G} R(\infty)
$$

(Proposition B.201). For the monoidal geometric fixed point functor, Proposition B.208 gives an isomorphism

$$
\Phi_{M}^{G}(R(\infty))=\Phi_{M}^{G}\left(M U_{c}^{((G))} \underset{A}{\wedge} S^{0}\right) \approx \Phi_{M}^{G} M U_{c}^{((G))} \underset{\Phi_{M}^{G} A}{\wedge} S^{0} .
$$

We next claim that there are associative algebra isomorphisms

$$
\Phi_{M}^{G} A \approx S^{0}\left[\Phi^{G} N \bar{r}_{1}, \Phi^{G} N \bar{r}_{2}, \ldots\right] \approx S^{0}\left[\Phi^{C_{2}} \bar{r}_{1}, \Phi^{C_{2}} \bar{r}_{2}, \ldots\right] .
$$

For the first, decompose $A$ into an indexed wedge, and use Proposition B.192. For the second use the fact that the monoidal geometric fixed point functor distributes over wedges, and for $V$ and $W$ representations of $C_{2}$, can be computed in terms of the isomorphisms

$$
\Phi_{M}^{G}\left(N_{C_{2}}^{G}\left(S^{-W} \wedge S^{V}\right)\right) \approx \Phi_{M}^{G}\left(S^{-\operatorname{ind}_{C_{2}}^{G} W} \wedge S^{\operatorname{ind}_{C_{2}}^{G} V}\right) \approx \Phi_{M}^{C_{2}}\left(S^{-W} \wedge S^{V}\right) .
$$

By Proposition B.202, $\Phi_{M}^{G} M U_{c}^{((G))}$ is a cofibrant $\Phi_{M}^{G} A$-module, and so

$$
\Phi_{M}^{G} M U_{c}^{((G))} \underset{\Phi_{M}^{G} A}{\wedge} S^{0} \approx \Phi_{M}^{G} M U^{((G))} /\left(\Phi_{M}^{G} N \bar{r}_{1}, \Phi_{M}^{G} N \bar{r}_{2}, \ldots\right) .
$$

Since $M U_{c}^{((G))}$ is a cofibrant $A$-module, and the polynomial algebra $A$ has the property that $S^{-1} \wedge A$ is cofibrant, the spectrum underlying $M U_{c}^{((G))}$ is cofibrant (Corollary B.207). This means that

$$
\Phi_{M}^{G} M U_{c}^{((G))}
$$

and

$$
\Phi^{G} M U_{c}^{((G))} \sim \Phi^{G} M U^{((G))} \sim M O
$$

are related by a functorial zig-zag of weak equivalences (Proposition B.201). Putting all of this together, we arrive at the equivalence

$$
\Phi^{G} R(\infty) \sim M O /\left(\Phi^{C_{2}} \bar{r}_{1}, \Phi^{C_{2}} \bar{r}_{2}, \ldots\right) .
$$


By Proposition 5.50

$$
\Phi^{G} \bar{r}_{i}= \begin{cases}h_{i} & i \neq 2^{k}-1 \\ 0 & i=2^{k}-1 .\end{cases}
$$

From this is an easy matter to compute $\pi_{*} M O /\left(\Phi^{G} \bar{r}_{1}, \Phi^{G} \bar{r}_{2}, \ldots\right)$ using the cofibration sequences described at the end of $§ 2.4 .3$. The outcome is as asserted.

Before going further we record a simple consequence of the above discussion which will be used in $\S 9.1$.

Proposition 7.6. The map

$$
\pi_{*} \Phi^{G} M U^{((G))}=\pi_{*} M O \rightarrow \pi_{*} \Phi^{G} H \underline{\mathbb{Z}}_{(2)}
$$

is zero for $*>0$.

A simple multiplicative property reduces the problem of showing that (7.4) is an isomorphism to showing that it is surjective in dimensions which are a power of 2 .

Lemma 7.7. If for every $k \geq 1$, the class $b^{2^{k-1}}$ is in the image of

$$
\pi_{2^{k}} \Phi^{G} M U^{((G))} /\left(G \cdot \bar{r}_{2^{k}-1}\right) \rightarrow \pi_{2^{k}} \Phi^{G} H \underline{\mathbb{Z}}_{(2)},
$$

then (7.4) is surjective, hence an isomorphism.

Proof: By writing

$$
R(\infty)=M U^{((G))} /\left(G \cdot \bar{r}_{1}\right) \bigwedge_{M U^{((G))}}^{\wedge} M U^{((G))} /\left(G \cdot \bar{r}_{2}\right) \bigwedge_{M U^{((G))}}^{\wedge} \cdots
$$

we see that if for every $k \geq 1, b^{2^{k-1}}$ is in the image of (7.8), then all products of the $b^{2^{k-1}}$ are in the image of

$$
\pi_{*} \Phi^{G} R(\infty) \rightarrow \pi_{*} \Phi^{G} H \underline{\mathbb{Z}}_{(2)} .
$$

Hence every power of $b$ is in the image of (7.9).

In view of Lemma 7.7, the Reduction Theorem follows from

Proposition 7.10. For every $k \geq 1$, the class $b^{2^{k-1}}$ is in the image of

$$
\pi_{2^{k}} \Phi^{G}\left(M U^{((G))} /\left(G \cdot \bar{r}_{2^{k}-1}\right)\right) \rightarrow \pi_{2^{k}} \Phi^{G}\left(H \underline{\mathbb{Z}}_{(2)}\right) .
$$

To simplify some of the notation, write

$$
c_{k}=2^{k}-1
$$

and

$$
M_{k}=M U^{((G))} /\left(G \cdot \bar{r}_{c_{k}}\right) \text {. }
$$

Since $S^{c_{k} \rho_{G}}$ is obtained from $S^{c_{k}}$ by attaching induced $G$-cells, the restriction map

$$
\pi_{c_{k} \rho_{G}+1}^{G} \tilde{E} \mathcal{P} \wedge M_{k} \rightarrow \pi_{c_{k}+1}^{G} \tilde{E} \mathcal{P} \wedge M_{k}
$$

is an isomorphism (Remark 2.50). The element of interest in this group (the one hitting $b^{2^{k-1}}$ ) arises from the class

$$
N \bar{r}_{c_{k}} \in \pi_{c_{k} \rho_{G}}^{G} M U^{((G))}
$$

and the fact that it is zero for two reasons in $\pi_{c_{k} \rho_{G}}^{G} \tilde{E} \mathcal{P} \wedge M_{k}$ (it has been coned off in the formation of $M_{k}$, and it is zero in $\pi_{c_{k} \rho_{G}}^{G} \tilde{E} \mathcal{P} \wedge M U^{((G))}=\pi_{c_{k}} M O$ by Proposition 5.50). We make this more precise and prove Proposition 7.10 by chasing 
the class $N \bar{r}_{c_{k}}$ around the sequences of equivariant homotopy groups arising from the diagram

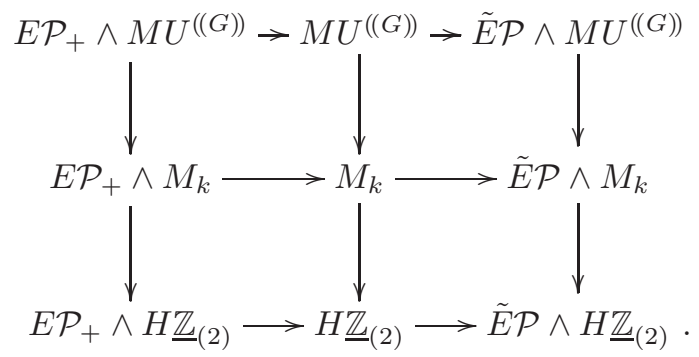

We start with the top row. By Proposition 5.50 the image of $N \bar{r}_{c_{k}}$ in

$$
\pi_{c_{k} \rho_{G}}^{G} \tilde{E} \mathcal{P} \wedge M U^{((G))} \approx \pi_{c_{k}}^{G} \tilde{E} \mathcal{P} \wedge M U^{((G))} \approx \pi_{c_{k}} M O
$$

is zero. There is therefore a class

$$
y_{k} \in \pi_{c_{k} \rho_{G}}^{G} E \mathcal{P}_{+} \wedge M U^{((G))}
$$

lifting $N \bar{r}_{c_{k}}$. The key computation, from which everything follows is

Proposition 7.12. The image under

$$
\pi_{c_{k} \rho_{G}}^{G} E \mathcal{P}_{+} \wedge M U^{((G))} \rightarrow \pi_{c_{k} \rho_{G}}^{G} E \mathcal{P}_{+} \wedge H \underline{\mathbb{Z}}_{(2)},
$$

of any choice of $y_{k}$ above, is non-zero.

Proof of Proposition 7.10 assuming Proposition 7.12: We continue chasing around the diagram (7.11). By construction the image of $y_{k}$ in $\pi_{c_{k} \rho_{G}}^{G} E \mathcal{P}_{+} \wedge M_{k}$ maps to zero in $\pi_{c_{k} \rho_{G}}^{G} M_{k}$. It therefore comes from a class

$$
\tilde{y}_{k} \in \pi_{c_{k} \rho_{G}+1}^{G} \tilde{E} \mathcal{P} \wedge M_{k} .
$$

The image of $\tilde{y}_{k}$ in $\pi_{c_{k} \rho_{G}+1}^{G} \tilde{E} \mathcal{P} \wedge H \underline{\mathbb{Z}}_{(2)}$ is non-zero since it has a non-zero image in

$$
\pi_{c_{k} \rho_{G}}^{G} E \mathcal{P}_{+} \wedge H \underline{\mathbb{Z}}_{(2)}
$$

by Proposition 7.12. Now consider the commutative square below, in which the horizontal maps are the isomorphisms (Remark 2.50) given by restriction along the fixed point inclusion $S^{2^{k}} \subset S^{c_{k} \rho_{G}+1}$ :

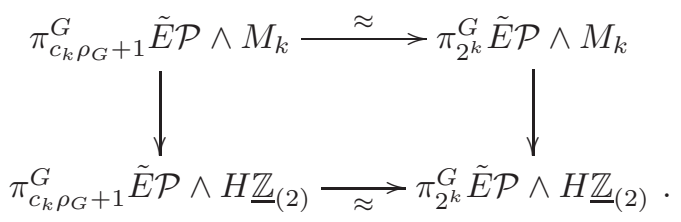

The group on the bottom right is cyclic of order 2, generated by $b^{2^{k-1}}$. We've just shown that the image of $\tilde{y}_{k}$ under the left vertical map is non-zero. It follows that the right vertical map is non-zero and hence that $b^{2^{k-1}}$ is in its image.

The remainder of this section is devoted to the proof of Proposition 7.12. 
The advantage of Proposition 7.12 is that it entirely involves $G$-spectra which have been smashed with $E \mathcal{P}_{+}$, and which (as discussed in $\S 7.2$ ) therefore fall under the jurisdiction of the induction hypothesis. In particular, the map

$$
E \mathcal{P}_{+} \wedge M U^{((G))} \rightarrow E \mathcal{P}_{+} \wedge H \underline{\mathbb{Z}}_{(2)}
$$

can be studied by smashing the slice tower of $M U^{((G))}$ with $E \mathcal{P}_{+}$.

We can cut down some the size of things by making use of the spectra introduced in $\S 7.2$. Factor (7.13) as

$$
E \mathcal{P}_{+} \wedge M U^{((G))} \rightarrow E \mathcal{P}_{+} \wedge R\left(c_{k}-1\right) \rightarrow E \mathcal{P}_{+} \wedge H \underline{\mathbb{Z}}_{(2)},
$$

and replace $y_{k}$ with its image

$$
y_{k} \in \pi_{c_{k} \rho_{G}}^{G} E \mathcal{P}_{+} \wedge R\left(c_{k}-1\right) .
$$

Lemma 7.14. For $0<m<c_{k} g$,

$$
\pi_{c_{k} \rho_{G}} E \mathcal{P}_{+} \wedge P_{m}^{m} R\left(c_{k}-1\right)=0 .
$$

There is an exact sequence

$$
\begin{aligned}
\pi_{c_{k} \rho_{G}}^{G} E \mathcal{P}_{+} \wedge P_{c_{k} g} R\left(c_{k}-1\right) \longrightarrow & \pi_{c_{k} \rho_{G}}^{G} E \mathcal{P}_{+} \wedge R\left(c_{k}-1\right) \\
& \downarrow \\
& \pi_{c_{k} \rho_{G}}^{G} E \mathcal{P}_{+} \wedge H \underline{\mathbb{Z}}_{(2)}=\mathbb{Z} / 2 .
\end{aligned}
$$

Proof: Because of the induction hypothesis, we know that the spectrum

$$
E \mathcal{P}_{+} \wedge P_{m}^{m} R\left(c_{k}-1\right)
$$

is contractible when $m$ is odd, and that when $m$ is even it is equivalent to

$$
E \mathcal{P}_{+} \wedge H \mathbb{Z} \wedge \widehat{W}_{m},
$$

where $\widehat{W} \subset S^{0}\left[G \cdot \bar{r}_{c_{k}}, \ldots\right]$ is the summand consisting of the wedge of slice cells of dimension $m$. Since $1<m<c_{k} g$ all of these cells are induced. This implies that the map

$$
E \mathcal{P}_{+} \wedge H \mathbb{Z} \wedge \widehat{W}_{m} \rightarrow H \mathbb{Z} \wedge \widehat{W}_{m}
$$

is an equivalence, since

$$
E \mathcal{P}_{+} \rightarrow S^{0}
$$

is an equivalence after restricting to any proper subgroup of $G$. But

$$
\pi_{c_{k} \rho_{G}}^{G} H \mathbb{Z} \wedge \widehat{W}_{m}=\pi_{0}^{G} H \mathbb{Z} \wedge S^{-c_{k} \rho_{G}} \wedge \widehat{W}_{m}=0
$$

since

$$
H \mathbb{Z} \wedge S^{-c_{k} \rho_{G}} \wedge \widehat{W}_{m}
$$

is an $\left(m-c_{k} g\right)$-slice and $m-c_{k} g<0$. This proves the first assertion. It implies that the map

$$
\pi_{c_{k} \rho_{G}}^{G} E \mathcal{P}_{+} \wedge P_{c_{k} g} R\left(c_{k}-1\right) \rightarrow \pi_{c_{k} \rho_{G}}^{G} E \mathcal{P}_{+} \wedge P_{1} R\left(c_{k}-1\right)
$$

is surjective. As mentioned in $\S 7.2$, Proposition 4.64 implies that $P_{0}^{0} R\left(c_{k}-1\right)=$ $H \mathbb{Z}_{(2)}$, and so the second assertion follows from the exact sequence of the fibration

$$
E \mathcal{P}_{+} \wedge P_{1} R\left(c_{k}-1\right) \rightarrow E \mathcal{P}_{+} \wedge R\left(c_{k}-1\right) \rightarrow E \mathcal{P}_{+} \wedge P_{0}^{0} R\left(c_{k}-1\right)
$$


The exact sequence in Lemma 7.14 converts the problem of showing that $y_{k}$ has non-zero image in $\pi_{c_{k} \rho_{G}}^{G} E \mathcal{P}_{+} \wedge H \underline{\mathbb{Z}}_{(2)}$ to showing that it is not in the image of

$$
\pi_{c_{k} \rho_{G}}^{G} E \mathcal{P}_{+} \wedge P_{c_{k} g} R\left(c_{k}-1\right)
$$

We now isolate a property of this image that is not shared by $y_{k}$. Recall that $\gamma$ is a fixed generator of $G$.

Proposition 7.15. The image of

$$
\pi_{c_{k} \rho_{G}}^{G} E \mathcal{P}_{+} \wedge P_{c_{k} g} R\left(c_{k}-1\right) \rightarrow \pi_{c_{k} \rho_{G}}^{G} R\left(c_{k}-1\right) \stackrel{i_{0}^{*}}{\longrightarrow} \pi_{c_{k} g}^{u} R\left(c_{k}-1\right)
$$

is contained in the image of $(1-\gamma)$.

The class $y_{k}$ does not have the property described in Proposition 7.15. Its image in $\pi_{c_{k} g}^{u} R\left(c_{k}-1\right)$ is $i_{0}^{*} N \bar{r}_{c_{k}}$ which generates a sign representation of $G$ occurring as a summand of $\pi_{c_{k} g}^{u} R\left(c_{k}-1\right)$. Thus once Proposition 7.15 is proved the proof of the Reduction Theorem is complete.

The proof of Proposition 7.15 makes use of the $R O(G)$-graded Mackey functor

$$
\underline{\pi}_{c_{k} \rho_{G}}(X)
$$

and the transfer map

$$
\underline{\pi}_{c_{k} \rho_{G}}(X)\left(C_{2}\right) \rightarrow \underline{\pi}_{c_{k} \rho_{G}}(X)(\mathrm{pt})
$$

in which $C_{2}$ is regarded as a finite $G$-set through the unique surjective map $G \rightarrow$ $C_{2}$. By definition (§3.1) of the covariant part $\underline{\pi}_{c_{k} \rho_{G *}}$ of the Mackey functor, the map (7.16) is given by the map of equivariant homotopy groups

$$
\pi_{c_{k} \rho_{G}}^{G}\left(X \wedge C_{2+}\right) \rightarrow \pi_{c_{k} \rho_{G}}^{G}(X)
$$

induced by the unique surjective map $C_{2} \rightarrow \mathrm{pt}$.

There are two steps in the proof of Proposition 7.15. First it is shown (Corollary 7.19) that the image of

$$
\pi_{c_{k} \rho_{G}}^{G} E \mathcal{P}_{+} \wedge P_{c_{k} g} R\left(c_{k}-1\right) \rightarrow \pi_{c_{k} \rho_{G}}^{G} R\left(c_{k}-1\right)
$$

is contained in the image of the transfer map just described. We then show (Lemma 7.20) that the image of the transfer map in $\pi_{c_{k} g}^{u} R\left(c_{k}-1\right)$ is in the image of $(1-\gamma)$.

Lemma 7.17. Let $M \geq 0$ be a $G$-spectrum, and regard $C_{2}$ as a finite $G$-set using the unique surjective map $G \rightarrow C_{2}$. The image of

$$
\pi_{0}^{G} E \mathcal{P}_{+} \wedge M \rightarrow \pi_{0}^{G} M
$$

is the image of the transfer map

$$
\pi_{0}^{G} M \wedge C_{2+} \rightarrow \pi_{0}^{G} M
$$

Proof: As mentioned in Remark 2.48, the space $E \mathcal{P}_{+}$can be taken to be the space $S_{+}^{\infty}$ on which $\gamma$ acts through the antipodal action. The standard cell decomposition in this model has 0 -skeleton $C_{2+}$. Since $M$ is $(-1)$-connected (Proposition 4.11) this implies that $\pi_{0}^{G} C_{2} \wedge M \rightarrow \pi_{0}^{G} E \mathcal{P}_{+} \wedge M$ is surjective, and the claim follows.

Corollary 7.18. The image of

$$
\pi_{c_{k} \rho_{G}}^{G} E \mathcal{P}_{+} \wedge P_{c_{k} g} R\left(c_{k}-1\right) \rightarrow \pi_{c_{k} \rho_{G}}^{G} P_{c_{k} g} R\left(c_{k}-1\right)
$$

is contained in the image of the transfer map. 
Proof: This follows from Lemma 7.17 above, after the identification

$$
\pi_{c_{k} \rho_{G}}^{G} P_{c_{k} g} R\left(c_{k}-1\right) \approx \pi_{0}^{G} S^{-c_{k} \rho_{G}} \wedge P_{c_{k} g} R\left(c_{k}-1\right)
$$

and the observation that

$$
S^{-c_{k} \rho_{G}} \wedge P_{c_{k} g} R\left(c_{k}-1\right) \approx P_{0}\left(S^{-c_{k} \rho_{G}} \wedge R\left(c_{k}-1\right)\right)
$$

is $\geq 0$.

Corollary 7.19. The image of

$$
\pi_{c_{k} \rho_{G}}^{G} E \mathcal{P}_{+} \wedge P_{c_{k} g} R\left(c_{k}-1\right) \rightarrow \pi_{c_{k} \rho_{G}}^{G} R\left(c_{k}-1\right)
$$

is contained in the image of the transfer map.

Proof: Immediate from Corollary 7.18 and the naturality of the transfer.

The remaining step is the special case $X=P_{c_{k} g} R\left(c_{k}-1\right), V=c_{k} \rho_{G}$ of the next result.

Lemma 7.20. Let $X$ be a $G$-spectrum, $V$ a virtual representation of $G$ of virtual dimension $d$, and regard $C_{2}$ as a finite $G$-set through the unique surjective map $G \rightarrow C_{2}$. Write $\epsilon \in\{ \pm 1\}$ for the degree of

$$
\gamma: i_{0}^{*} S^{V} \rightarrow i_{0}^{*} S^{V}
$$

The image of

$$
\pi_{V}^{G}\left(X \wedge C_{2+}\right) \rightarrow \pi_{V}^{G} X \rightarrow \pi_{d}^{u} X
$$

is contained in the image of

$$
(1+\epsilon \gamma): \pi_{d}^{u} X \rightarrow \pi_{d}^{u} X .
$$

Proof: Consider the diagram

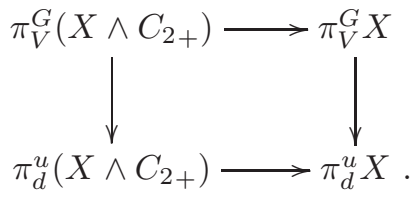

The non-equivariant identification

$$
C_{2+} \approx S^{0} \vee S^{0}
$$

gives an isomorphism of groups of non-equivariant stable maps

$$
\left[S^{V}, X \wedge C_{2+}\right] \approx\left[S^{V}, X\right] \oplus\left[S^{V}, X\right],
$$

and so an isomorphism of the group in the lower left hand corner with

$$
\pi_{d}^{u} X \oplus \pi_{d}^{u} X
$$

under which the generator $\gamma \in G$ acts as

$$
(a, b) \mapsto(\epsilon \gamma b, \epsilon \gamma a) .
$$

The map along the bottom is $(a, b) \mapsto a+b$. Now the image of the left vertical map is contained in the set of elements invariant under $\gamma$ which, in turn, is contained in the set of elements of the form

The result follows.

$$
(a, \epsilon \gamma a) .
$$


Proof of Proposition \%.15: As described after its statement, Proposition 7.15 is a consequence of Corollary 7.19 and Lemma 7.20.

\section{The Gap Theorem}

The proof of the Gap Theorem was sketched in the introduction, and various supporting details were scattered throughout the paper. We collect the story here for convenient reference.

Given the Slice Theorem, the Gap Theorem is a consequence of the following special case of Proposition 3.20

Proposition 8.1. Suppose that $G=C_{2^{n}}$ is a non-trivial group, and $m \geq 0$. Then

$$
H_{G}^{i}\left(S^{m \rho_{G}} ; \underline{\mathbb{Z}}_{(2)}\right)=0 \quad \text { for } 0<i<4 .
$$

Lemma 8.2 (The Cell Lemma). Let $G=C_{2^{n}}$ for some $n>0$. If $\widehat{S}$ is an isotropic slice cell of even dimension, then the groups $\pi_{k}^{G} H \underline{\mathbb{Z}}_{(2)} \wedge \widehat{S}$ are zero for $-4<k<0$.

Proof: Suppose that

$$
\widehat{S}=G_{+}{ }_{H}^{\wedge} S^{m \rho_{H}}
$$

with $H \subset G$ non-trivial. By the Wirthmüller isomorphism

$$
\pi_{k}^{G} H \underline{\mathbb{Z}}_{(2)} \wedge \widehat{S} \approx \pi_{k}^{H} H \underline{\mathbb{Z}}_{(2)} \wedge S^{m \rho_{H}},
$$

so the assertion is reduced to the case $\widehat{S}=S^{m \rho_{G}}$ with $G$ non-trivial. If $m \geq 0$ then $\pi_{k}^{G} H \underline{\mathbb{Z}}_{(2)} \wedge \widehat{S}=0$ for $k<0$. For the case $m<0$ write $i=-k, m^{\prime}=-m>0$, and

$$
\pi_{k}^{G} H \underline{\mathbb{Z}}_{(2)} \wedge \widehat{S}=H_{G}^{i}\left(S^{m^{\prime} \rho_{G}} ; \underline{\mathbb{Z}}_{(2)}\right) .
$$

The result then follows from Proposition 8.1.

Theorem 8.3. If $X$ is pure and isotropic, then

$$
\pi_{i}^{G} X=0 \quad-4<i<0 .
$$

Proof: Immediate from the slice spectral sequence for $X$ and the Cell Lemma.

Corollary 8.4. If $Y$ can be written as a directed homotopy colimit of isotropic pure spectra, then

$$
\pi_{i}^{G} X=0 \quad-4<i<0 .
$$

Theorem 8.5 (The Gap Theorem). Let $G=C_{2^{n}}$ with $n>0$ and $D \in \pi_{\ell_{G}} M U^{((G))}$ be any class. Then for $-4<i<0$

$$
\pi_{i}^{G} D^{-1} M U^{((G))}=0 .
$$


Proof: The spectrum $D^{-1} M U^{((G))}$ is the homotopy colimit

$$
\underset{j}{\operatorname{holim}} \Sigma^{-j \ell \rho_{G}} M U^{((G))} \text {. }
$$

By the Slice Theorem, $M U^{((G))}$ is pure and isotropic. But then the spectrum

$$
\Sigma^{-j \ell \rho_{G}} M U^{((G))}
$$

is also pure and isotropic, since for any $X$

$$
P_{m}^{m} \Sigma^{\rho_{G}} X \approx \Sigma^{\rho_{G}} P_{m-g}^{m-g} X
$$

by Corollary 4.25. The result then follows from Corollary 8.4.

\section{The Periodicity Theorem}

In this section we will describe a general method for producing periodicity results for spectra obtained from $M U^{((G))}$ by inverting suitable elements of $\pi_{\star}^{G} M U^{((G))}$. The Periodicity Theorem (Theorem 9.19) used in the proof of Theorem 1.1 is a special case. The proof relies on a small amount of computation of $\pi_{\star}^{G} M U^{((G))}$.

9.1. The $R O(G)$-graded slice spectral sequence for $M U^{((G))}$. Let $\sigma=\sigma_{G}$ be the real sign representation of $G$, and

$$
u=u_{2 \sigma} \in \pi_{2-2 \sigma}^{G} H \underline{\mathbb{Z}}_{(2)}
$$

the element defined in Definition 3.12. Since

$$
P_{0}^{0} M U^{((G))}=H \underline{\mathbb{Z}}
$$

the powers $u^{m}$ define elements

$$
u^{m} \in E_{2}^{0,2 m-2 m \sigma}=\pi_{2 m-2 m \sigma}^{G} P_{0}^{0} M U^{((G))}
$$

in the $E_{2}$-term of the $R O(G)$-graded slice spectral sequence

$$
E_{2}^{s, t}=\pi_{t-s}^{G} P_{\operatorname{dim} t}^{\operatorname{dim} t} M U^{((G))} \Longrightarrow \pi_{t-s} M U^{((G))},
$$

with $t \in-2 m \sigma+\mathbb{Z}$. Our periodicity theorems depend on the fate of these elements. To study them it is convenient to consider odd negative multiples of $\sigma$ as well, and to investigate the slice spectral sequences for $\pi_{*-k \sigma}$ for $k \geq 0$.

It turns out to be enough to investigate the groups $E_{2}^{s, t}$ with $s \geq(g-1)((t-$ $s)-(k-k \sigma))$, where $g=|G|$. The situation is depicted in Figures 2-4. We have, in fact, already described all of the groups in this range. To see this write $t^{\prime}=\operatorname{dim} t$ so that $t=t^{\prime}+(k-k \sigma)$, and

$$
E_{2}^{s, t}=\pi_{t^{\prime}-s+k}^{G} S^{k \sigma} \wedge P_{t^{\prime}}^{t^{\prime}} M U^{((G))} .
$$

Since $S^{k \sigma} \wedge P_{t^{\prime}}^{t^{\prime}} M U^{((G))} \geq t^{\prime}$, part iii) of Proposition 4.40 tells us that this group vanishes if

and hence if

$$
t^{\prime}-s+k<\left\lfloor t^{\prime} / g\right\rfloor
$$

$$
s>(g-1)((t-s)+k \sigma)+k .
$$

This gives the vanishing line depicted in Figures 2-4. Now $P_{t^{\prime}}^{t^{\prime}} M U^{((G))}$ is contractible unless $t^{\prime}$ is even, in which case it a wedge of $G$-spectra of the form $H \underline{\mathbb{Z}} \wedge \widehat{S}$ where 


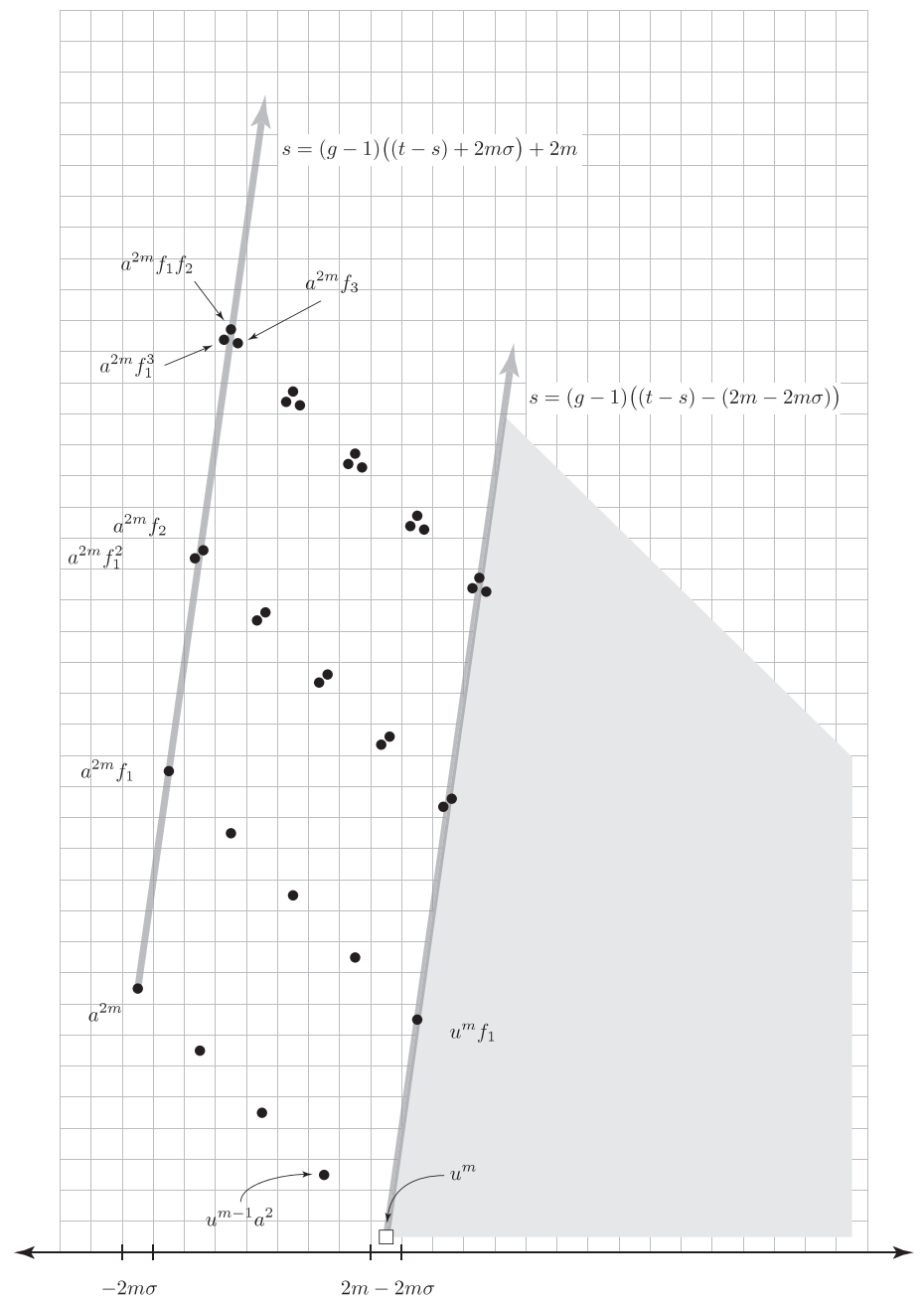

FIGURE 2. The slice spectral sequence for $\pi_{-2 m \sigma+*}^{G} M U^{((G))}$

$\widehat{S}$ is a slice cell of dimension $t^{\prime}$. Since the restriction of $\sigma$ to any proper subgroup is trivial, when $\widehat{S}=G_{+}{ }_{H} S^{\ell^{\prime} \rho_{H}}$ is an induced slice cell, there are isomorphisms

$$
S^{k \sigma} \wedge H \underline{\mathbb{Z}} \wedge \widehat{S} \approx G_{+}{ }_{H}\left(S^{k \sigma} \wedge H \underline{\mathbb{Z}} \wedge S^{\ell^{\prime} \rho_{H}}\right) \approx G_{+}{ }_{H}\left(S^{k} \wedge H \underline{\mathbb{Z}} \wedge S^{\ell^{\prime} \rho_{H}}\right)
$$

an so $\pi_{t^{\prime}-s+k}^{G} S^{k \sigma} \wedge H \underline{\mathbb{Z}} \wedge \widehat{S}$ is isomorphic to

$$
\pi_{t^{\prime}-s}^{H} H \underline{\mathbb{Z}} \wedge S^{\ell^{\prime} \rho_{H}} .
$$

This group vanishes if

$$
t^{\prime}-s<\ell^{\prime}=t^{\prime} / h \quad(h=|H|),
$$

so certainly when

$$
t^{\prime}-s \leq t^{\prime} / g
$$




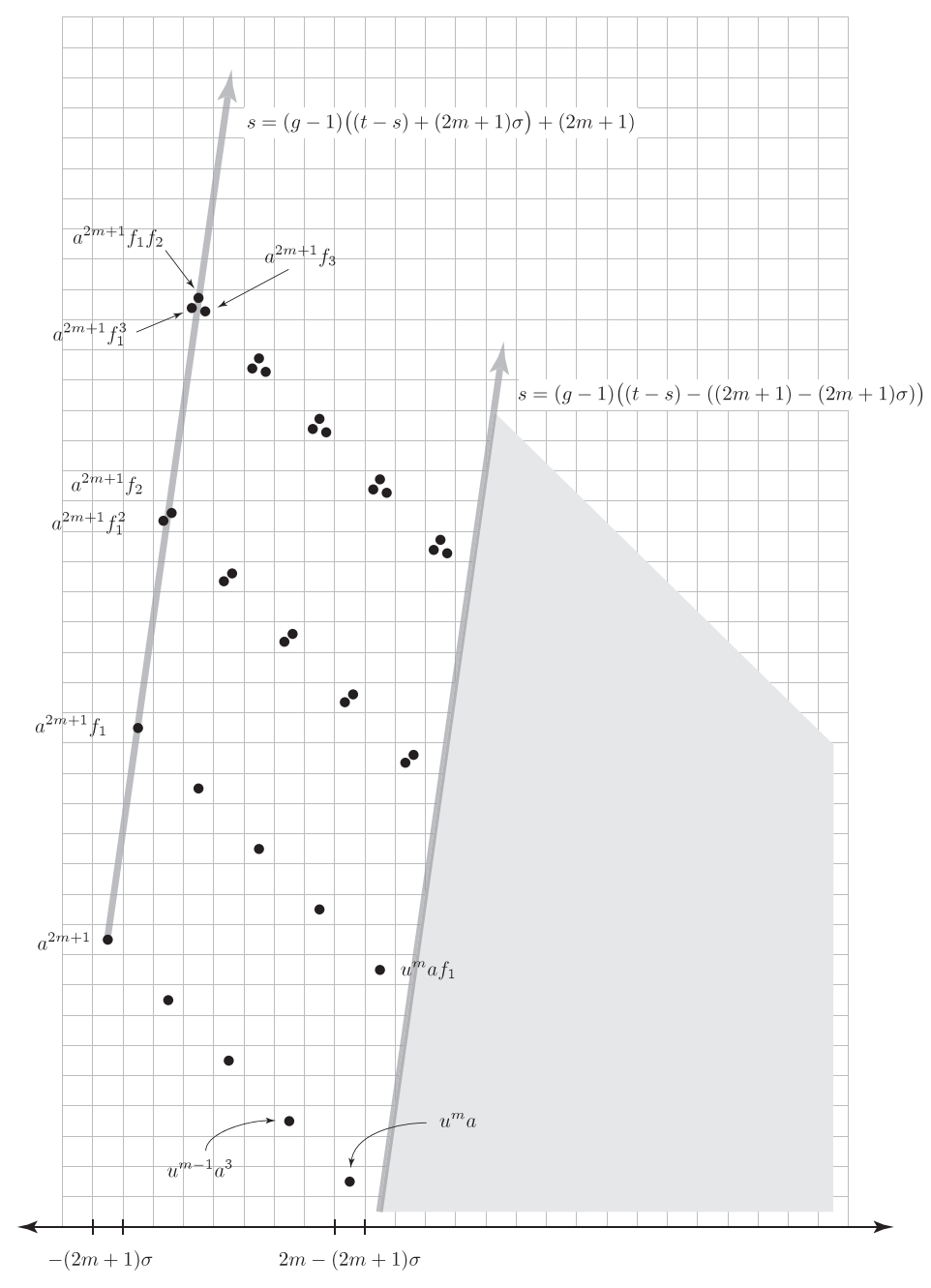

Figure 3. The slice spectral sequence for $\pi_{-(2 m+1) \sigma+*}^{G} M U^{((G))}$

or, equivalently when

$$
s \geq(g-1)((t-s)-(k-k \sigma)) .
$$

Thus in this range only the non-induced slice cells contribute.

The only even dimensional slice cells which are not induced are those of the form $S^{\ell \rho_{G}}$. We are therefore studying the groups

$$
\pi_{j}^{G} H \underline{\mathbb{Z}} \wedge S^{k \sigma} \wedge S^{\ell \rho_{G}}
$$

with $j \leq \ell+k$ and $k, \ell \geq 0$.

Lemma 9.1. For $k, \ell \geq 0$ and $j \leq \ell+k$ the group

$$
\pi_{j}^{G} H \underline{\mathbb{Z}} \wedge S^{k \sigma} \wedge S^{\ell \rho_{G}}
$$




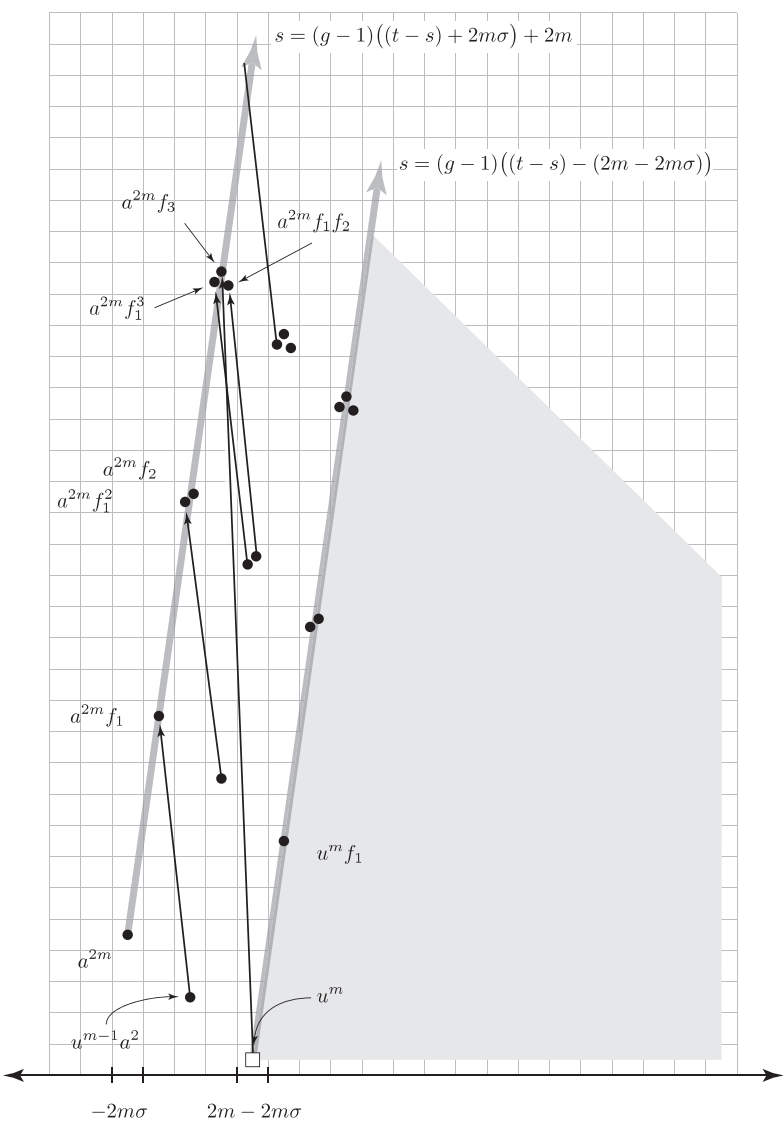

FiguRE 4. Differentials on $u^{m}$

is given by

$$
\pi_{j}^{G} H \underline{\mathbb{Z}} \wedge S^{k \sigma} \wedge S^{\ell \rho_{G}} \approx \begin{cases}0 & \text { if }(j-\ell)<0 \text { or }(j-\ell) \text { is odd } \\ \mathbb{Z} / 2 \cdot\left\{a_{\bar{\rho}}^{\ell} a_{\sigma}^{k-2 m} u_{2 \sigma}^{m}\right\} & \text { if }(j-\ell)=2 m \geq 0 \text { and } \ell>0 \\ \mathbb{Z}_{(2)} \cdot\left\{u_{2 \sigma}^{m}\right\} & \text { if }(j-\ell)=2 m \geq 0 \text { and } \ell=0 .\end{cases}
$$

Proof: This computation reduces to the one described in Example 3.16. To see this, write

$$
S^{k \sigma} \wedge S^{\ell \rho_{G}}=S^{(k+\ell) \sigma} \wedge S^{\ell} \wedge S^{\ell\left(\rho_{G}-\sigma-1\right)},
$$

and consider the map

$$
a_{\bar{\rho}-\sigma}^{\ell}: \pi_{j}^{G} H \underline{\mathbb{Z}} \wedge S^{(k+\ell) \sigma} \wedge S^{\ell} \rightarrow \pi_{j}^{G} H \underline{\mathbb{Z}} \wedge S^{k \sigma} \wedge S^{\ell \rho_{G}} .
$$

given by multiplication by $a_{\bar{\rho}-\sigma}^{\ell}$. When $\ell=0$ this map it is an isomorphism. When $\ell>0$, the space $S^{\ell\left(\rho_{G}-\sigma-1\right)}$ has the structure of a $G$-CW complex with one 0-cell and all other $G$-cells of positive dimension and induced from proper subgroups. Smashing with $S^{(k+\ell) \sigma}$ and using the fact that the restriction of $\sigma$ to every proper subgroup of $G$ is trivial, one finds that $S^{k \sigma} \wedge S^{\ell \rho_{G}}$ is obtained from $S^{(k+\ell) \sigma} \wedge S^{\ell}$ 
by attaching induced $G$-cells of dimension greater than $(k+2 \ell)$. This implies that the map $a_{\bar{\rho}-\sigma}^{\ell}$ is an isomorphism for $j<k+2 \ell$, and so certainly for $j \leq k+\ell$ since $\ell>0$. Thus in the range of interest, multiplication by $a_{\bar{\rho}-\sigma}^{\ell}$ is isomorphism, and the computation reduces to the evaluation of

$$
\pi_{j}^{G} H \underline{\mathbb{Z}} \wedge S^{(k+\ell) \sigma} \wedge S^{\ell}
$$

These groups were described in Example 3.16.

To complete the description of the $E_{2}$-term of the $R O(G)$-graded slice spectral sequence in this range we need to identify the summand of non-induced slices of $M U^{((G))}$. From the associative algebra equivalence

$$
\bigvee_{k \in \mathbb{Z}} P_{k}^{k} M U^{((G))} \sim H \mathbb{Z} \wedge S^{0}\left[G \cdot \bar{r}_{1}, \ldots\right]
$$

this is equivalent to identifying the summand of non-induced slice cells in the twisted monoid ring

$$
S^{0}\left[G \cdot \bar{r}_{1}, \ldots\right] \text {. }
$$

Since the smash product of an induced spectrum with any spectrum is induced, we can do this by identifying the summand of non-induced slice cells in each

$$
S^{0}\left[G \cdot \bar{r}_{i}\right]
$$

and smashing them together.

Take the generating inclusion

$$
\bar{r}_{i}: S^{i \rho_{C_{2}}} \rightarrow S^{0}\left[\bar{r}_{i}\right]
$$

apply $N_{C_{2}}^{G}$ to obtain

$$
N \bar{r}_{i}: S^{i \rho_{G}} \rightarrow S^{0}\left[G \cdot \bar{r}_{i}\right],
$$

and extend it to an associative algebra map

$$
S^{0}\left[N \bar{r}_{i}\right] \rightarrow S^{0}\left[G \cdot \bar{r}_{i}\right]
$$

Lemma 9.4. The map (9.3) is the inclusion of the summand of non-induced slice cells.

Proof: The distributive law expresses $S^{0}\left[G \cdot \bar{r}_{i}\right]=N_{C_{2}}^{G} S^{0}\left[\bar{r}_{i}\right]$ as an indexed wedge (see $\S 2.4)$

$$
S^{0}\left[G \cdot \bar{r}_{i}\right] \approx \bigvee_{f: G / C_{2} \rightarrow \mathbb{N}_{0}} S^{V_{f}}
$$

and $V_{f}=\bigoplus_{i=1}^{g / 2} \gamma^{i} f\left(\gamma^{i}\right) \rho_{C_{2}}$. We now decompose the right hand side into an ordinary wedge over the $G$-orbits. Since an indexed wedge over a $G$-orbit is induced from the stabilizer of any element of the orbit, the summand of non-induced slice cells consists of those $f$ which are constant. If $f: G / C_{2}$ is the constant function with value $n$, then $V_{f}=n \rho_{G}$, so the summand of non-induced slice cells is

$$
\bigvee_{\underline{n}} S^{n \rho_{G}} \text {. }
$$

The result follows easily from this.

Smashing these together gives 
Corollary 9.5. The associative algebra map

$$
S^{0}\left[N \bar{r}_{1}, \ldots\right] \rightarrow S^{0}\left[G \cdot \bar{r}_{1}, \ldots\right]
$$

is the inclusion of the summand of non-induced slice cells.

To put this all together, consider the $\mathbb{Z} \times R O(G)$-graded ring

$$
\mathbb{Z}_{(2)}\left[a, f_{i}, u\right] /\left(2 a, 2 f_{i}\right)
$$

with

$$
\begin{aligned}
|a| & =(1,1-\sigma) \\
\left|f_{i}\right| & =(i(g-1), i g) \\
|u| & =(0,2-2 \sigma) .
\end{aligned}
$$

Define a map

$$
\mathbb{Z}_{(2)}\left[a, f_{i}, u\right] /\left(2 a, 2 f_{i}\right) \rightarrow \bigoplus_{\substack{s, k \geq 0 \\ t \in *-k \sigma}} E_{2}^{s, t}
$$

by

$$
\begin{aligned}
f_{i} & \mapsto a_{\bar{\rho}}^{i} N \bar{r}_{i} \in E_{2}^{i(g-1), i g}=\pi_{i}^{G} P_{i g}^{i g} M U^{((G))} \\
a & \mapsto a_{\sigma} \in E_{2}^{1,1-\sigma}=\pi_{-\sigma} P_{0}^{0} 0 M U^{((G))}
\end{aligned}
$$

and by sending $u$ to the element $u \in E_{2}^{0,2-2 \sigma}$ described at the beginning of this section. The combination of Lemmas 9.1 and 9.4 gives

Proposition 9.7. The map

$$
\mathbb{Z}_{(2)}\left[a, f_{i}, u\right] /\left(2 a, 2 f_{i}\right) \rightarrow \bigoplus_{\substack{s, k \geq 0 \\ t \in *-k \sigma}} E_{2}^{s, t}
$$

is an isomorphism in the range

$$
s \geq(g-1)((t-s)-(k-k \sigma)) .
$$

We now turn to the differentials. By construction, the $f_{i}$ are the representatives at the $E_{2}$-term of the slice spectral sequence of the elements defined in Definition 5.51 (and also called $f_{i}$ ). They are therefore permanent cycles. Similarly, the element $a$ is the representative of $a_{\sigma}$ and also a permanent cycle. This leaves the powers of $u$. The case $G=C_{2}$ of the following result appears in unpublished work of Araki and in Hu-Kriz [35].

Theorem 9.9 (Slice Differentials Theorem). In the slice spectral sequence for $\pi_{\star}^{G} M U^{((G))}$ the differentials $d_{i} u^{2^{k-1}}$ are zero for $i<r=1+\left(2^{k}-1\right) g$, and

$$
d_{r} u^{2^{k-1}}=a^{2^{k}} f_{2^{k}-1} .
$$

Remark 9.10. It follows from Proposition 9.7 that what lies on the "vanishing line"

$$
s=(g-1)((t-s)+k \sigma)+k
$$

is the algebra

$$
\mathbb{Z}_{(2)}\left[a, f_{i}\right] /\left(2 a, 2 f_{i}\right)
$$


In Proposition 5.50 it was shown that the kernel of the map

$$
\mathbb{Z}_{(2)}\left[a_{\sigma}, f_{i}\right] /\left(2 a, 2 f_{i}\right) \rightarrow \pi_{\star}^{G} M U^{((G))} \rightarrow \pi_{\star}^{G} \Phi^{G} M U^{((G))}=\pi_{*} M O\left[a_{\sigma}^{ \pm 1}\right]
$$

is the ideal $\left(2, f_{1}, f_{3}, f_{7}, \ldots\right)$. The only possible non-trivial differentials into the vanishing line must therefore land in this ideal.

For the proof of Theorem 9.9 the reader may find it helpful to consult Figure 4.

Proof of Theorem 9.9: We establish the differential by induction on $k$. Assume the result for $k^{\prime}<k$. Then what's left in the range $s \geq(g-1)(t-s-k)$ after the differentials assumed by induction is the sum of two modules over $\mathbb{Z}_{(2)}\left[f_{i}\right] /\left(2 f_{i}\right)$. One is generated by $a^{2^{k}}$ and is free over the quotient ring

$$
\mathbb{Z} / 2\left[f_{i}\right] /\left(f_{1}, f_{3}, \ldots, f_{2^{k-1}-1}\right) .
$$

The other is generated by $u^{2^{k-1}}$. Since the differential must take its value in the ideal $\left(2, a, f_{1}, f_{3}, \ldots\right)$, the next (and only) possible differential on $u^{2^{k-1}}$ is the one asserted in the theorem. So all we need do is show that the classes $u^{2^{k-1}}$ do not survive the spectral sequence. For this it suffices to do so after inverting $a$. Consider the map

$$
a_{\sigma}^{-1} \pi_{\star}^{G} M U^{((G))} \rightarrow a_{\sigma}^{-1} \pi_{\star}^{G} H \underline{\mathbb{Z}}_{(2)} .
$$

We know the $\mathbb{Z}$-graded homotopy groups of both sides, since they can be identified with the homotopy groups of the geometric fixed point spectrum. If $u^{2^{k-1}}$ is a permanent cycle, then the class $a^{-2^{k}} u^{2^{k-1}}$ is as well, and represents a class with non-zero image in $\pi_{*}^{G} \Phi^{G} H \underline{\mathbb{Z}}_{(2)}$. This contradicts Proposition 7.6.

Remark 9.11. After inverting $a_{\sigma}$, the differentials described in Theorem 9.9 describe completely the $R O(G)$-graded slice spectral sequence. The spectral sequence starts from

$$
\mathbb{Z} / 2\left[f_{i}, a^{ \pm 1}, u\right]
$$

The class $u^{2^{k-1}}$ hits a unit multiple of $f_{2^{k}-1}$, and so the $E_{\infty}$-term is

$$
\mathbb{Z} / 2\left[f_{i}, i \neq 2^{k}-1\right]\left[a^{ \pm 1}\right]=M O_{*}\left[a^{ \pm 1}\right]
$$

which we know to be the correct answer since $\Phi^{G} M U^{((G))}=M O$. This also shows that the class $u^{2^{k-1}}$ is a permanent cycle modulo $\left(\bar{r}_{2^{k}-1}\right)$. This fact corresponds to the main computation in the proof of Theorem 6.5 (which, of course we used in the above proof). The logic can be reversed, and in [35] the results are established in the reverse order (for the group $G=C_{2}$ ).

Write

$$
\overline{\mathfrak{d}}_{k}=N \bar{r}_{2^{k}-1} \in \pi_{\left(2^{k}-1\right) \rho_{G}}^{G} M U^{((G))},
$$

and note that with this notation

$$
f_{2^{k}-1}=a_{\bar{\rho}}^{2^{k}-1} \overline{\mathfrak{d}}_{k}
$$

In the proof of the corollary below we will make use of the identity

$$
f_{2^{k+1}-1} \overline{\mathfrak{d}}_{k}=a_{\bar{\rho}}^{2^{k+1}-1} \overline{\mathfrak{d}}_{k+1} \overline{\mathfrak{d}}_{k}=f_{2^{k}-1} a_{\bar{\rho}}^{2^{k}} \overline{\mathfrak{d}}_{k+1}
$$

The map $\overline{\mathfrak{d}}_{k}: S^{\left(2^{k}-1\right) \rho_{G}} \rightarrow M U^{((G))}$ is represented at the $E_{2}$-term of the $R O(G)$ graded slice spectral sequence by a map $S^{\left(2^{k}-1\right) \rho_{G}} \rightarrow P_{\left(2^{k-1}\right) g}^{\left(2^{k-1}\right) g} M U^{((G))}$ which we 
will also call $\overline{\mathfrak{d}}_{k}$. Multiplying, this defines elements $\overline{\mathfrak{d}}_{k} u^{2^{k}}$ in the $E_{2}$-term of the $R O(G)$-graded slice spectral sequence.

Corollary 9.13. In the $R O(G)$-graded slice spectral sequence for $M U^{((G))}$, the class $\overline{\mathfrak{d}}_{k} u^{2^{k}}$ is a permanent cycle.

Proof: Write

$$
r=1+\left(2^{k+1}-1\right) g .
$$

Theorem 9.9 implies that differentials $d_{i}\left(\overline{\mathfrak{d}}_{k} u^{2^{k}}\right)=\overline{\mathfrak{d}}_{k} d_{i}\left(u^{2^{k}}\right)$ are zero for $i<r$, and

$$
d_{r}\left(\overline{\mathfrak{d}}_{k} u^{2^{k}}\right)=\overline{\mathfrak{d}}_{k} a^{2^{k+1}} f_{2^{k+1}-1}=a^{2^{k+1}} f_{2^{k}-1} a_{\bar{\rho}}^{2^{k}} \overline{\mathfrak{d}}_{k+1},
$$

the second equality coming from (9.12) above. But from the earlier differential

$$
d_{r^{\prime}} u^{2^{k-1}}=a^{2^{k}} f_{2^{k}-1}
$$

where $r^{\prime}=1+\left(2^{k}-1\right) g<r$, we also have

$$
d_{r^{\prime}}\left(u^{2^{k-1}} a^{2^{k}} a_{\bar{\rho}}^{2^{k}} \overline{\mathfrak{d}}_{k+1}\right)=a^{2^{k+1}} f_{2^{k}-1} a_{\bar{\rho}}^{2^{k}} \overline{\mathfrak{d}}_{k+1}
$$

so that in fact $d_{r}\left(\overline{\mathfrak{d}}_{k} u^{2^{k}}\right)=0$. The target of the remaining differentials work out to be in a region of the spectral sequence which is already zero at the $E_{2}$-term. So once we check this, the proof is complete.

To check the claim about the vanishing region first note that with our conventions, differential $d_{i+1}$ of the $R O(G)$-graded slice spectral sequence maps a subquotient of

to a sub-quotient of

$$
\pi_{m}^{G} P_{n}^{n} X
$$

$$
\pi_{m-1}^{G} P_{n+i}^{n+i} X
$$

The class in question starts out at the $E_{2}$-term as

$$
\overline{\mathfrak{d}}_{k} u^{2^{k}} \in \pi_{2^{k}(2-2 \sigma)+\left(2^{k}-1\right) \rho_{G}}^{G} P_{\left(2^{k}-1\right) g}^{\left(2^{k}-1\right) g} M U^{((G))}
$$

so we are interested in the groups

$$
\pi_{2^{k}(2-2 \sigma)+\left(2^{k}-1\right) \rho_{G}-1}^{G} P_{\left(2^{k}-1\right) g+i}^{\left(2^{k}-1\right) g+i} M U^{((G))}
$$

or, equivalently

$$
\pi_{2^{k+1}-1}^{G}\left(S^{2^{k+1} \sigma} \wedge S^{-\left(2^{k}-1\right) \rho_{G}} \wedge P_{\left(2^{k}-1\right) g+i}^{\left(2^{k}-1\right) g+i} M U^{((G))}\right)
$$

with $i+1>r=1+\left(2^{k+1}-1\right) g$. To simplify the notation, write

$$
X_{i}=S^{-\left(2^{k}-1\right) \rho_{G}} \wedge P_{\left(2^{k}-1\right) g+i}^{\left(2^{k}-1\right) g+i} M U^{((G))},
$$

so that the group we are interested in is

$$
\pi_{2^{k+1}-1}^{G}\left(S^{2^{k+1} \sigma} \wedge X_{i}\right)
$$

Now

$$
X_{i} \geq i .
$$

so Proposition 4.40 implies that

$$
\pi_{j}^{G} X_{i}=0
$$

for $j<\lfloor i / g\rfloor$. Since $S^{2^{k+1} \sigma}$ is $(-1)$-connected this means that if $i \geq 2^{k+1} g$ the group (9.14) is trivial. The remaining values of $i$ are strictly between $\left(2^{k+1}-1\right) g$ 
and $\left(2^{k+1}\right) g$, and hence not divisible by $g$. But since $M U^{((G))}$ is pure, when $i$ is not

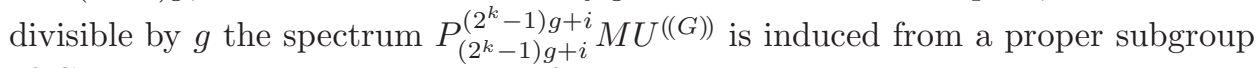
of $G$, hence so is $X_{i}$. There is therefore an equivalence

$$
S^{2^{k+1} \sigma} \wedge X_{i} \approx S^{2^{k+1}} \wedge X_{i}
$$

and so

since $X_{i} \geq 0$.

$$
\pi_{2^{k+1}-1}^{G}\left(S^{2^{k+1} \sigma} \wedge X_{i}\right)=\pi_{2^{k+1}-1}^{G}\left(S^{2^{k+1}} \wedge X_{i}\right)=0
$$

9.2. Periodicity theorems. We now turn to our main periodicity theorem. As will be apparent to the reader, the technique can be used to get a much more general result. We have chosen to focus on a case which contains what is needed for the proof of Theorem 1.1, and yet can be stated for general $G=C_{2^{n}}$.

Our motivating example is the spectrum $K_{\mathbb{R}}$ of "real" $K$-theory [7]. Multiplication by the real Bott class $\bar{r}_{1} \in \pi_{\rho_{2}} K_{\mathbb{R}}$ is an isomorphism, giving $K_{\mathbb{R}}$ an $S^{\rho_{2}}$ periodicity. On the other hand, the representation $4 \rho_{2}$ admits a Spin structure, and the construction of the $K O$-orientation of Spin bundles leads to a "Thom" class $u \in \pi_{8}^{C_{2}} K_{\mathbb{R}} \wedge S^{4 \rho_{2}}$. This class is represented at the $E_{2}$-term of the slice spectral sequence by $u_{4 \rho_{2}}$. Multiplication by $\bar{r}_{1}^{4} u$ is then an equivariant map $S^{8} \wedge K_{\mathbb{R}} \rightarrow K_{\mathbb{R}}$ whose underlying map of non-equivariant spectra is an equivalence. It therefore gives an equivalence $S^{8} \wedge K_{\mathbb{R}}^{h C_{2}} \approx K_{\mathbb{R}}^{h C_{2}}$. Since the map $K O \rightarrow K_{\mathbb{R}}^{h C_{2}}$ is an equivalence, this gives the 8-fold periodicity of $K O$.

In our situation we begin with an equivariant commutative ring $R$, a representation $V$ of $G$, and an element $D \in \pi_{V}^{G} R$. We manually create a spectrum with $S^{V}$-periodicity by working with the homotopy colimit, $D^{-1} R$, of the sequence

$$
R \stackrel{D}{\rightarrow} S^{-V} \wedge R \stackrel{D}{\rightarrow} S^{-2 V} \wedge R \rightarrow \cdots .
$$

The unit inclusion

$$
S^{0} \rightarrow D^{-1} R
$$

gives a map

$$
H \underline{\mathbb{Z}}=P_{0}^{0} S^{0} \rightarrow P_{0}^{0} D^{-1} R
$$

and hence defines, for every oriented representation $W$ of $G$, elements

$$
u_{W} \in \pi_{\operatorname{dim} W-W} P_{0}^{0} R=E_{2}^{0, \operatorname{dim} W-W}
$$

in the $E_{2}$-term of the $R O(G)$-graded slice spectral sequence for $\pi_{\star}^{G} D^{-1} R$. We will show, under certain hypotheses on $D$, that there is an integer $k>0$ with the property that $u_{k V}$ is a permanent cycle. Let $u \in \pi_{\star}^{G} D^{-1} R$ be any element representing $u_{k V}$. Then the equivariant map

$$
S^{k \operatorname{dim} V} \wedge D^{-1} R \stackrel{u}{\rightarrow} S^{k V} \wedge D^{-1} R \stackrel{D^{k}}{\longrightarrow} D^{-1} R
$$

induces an equivalence of underlying, non-equivariant spectra, and hence an equivalence of homotopy fixed point spectra

$$
\left(S^{k \operatorname{dim} V} \wedge D^{-1} R\right)^{h G} \rightarrow\left(D^{-1} R\right)^{h G} .
$$

This establishes a periodicity theorem for the homotopy fixed point spectrum $\left(D^{-1} R\right)^{h G}$.

The exposition is cleanest when one exploits multiplicative properties of the spectrum $D^{-1} R$. There are some easy general things to say at first. The spectrum 
$D^{-1} R$ is certainly an $R$-module, and inherits a homotopy commutative multiplication (over $R$ ) from $R$. The technique of [22, §VIII.4] can be used to show that the non-equivariant spectrum underlying $D^{-1} R$ has a unique commutative algebra structure for which the map $i_{0}^{*} R \rightarrow i_{0}^{*} D^{-1} R$ is a map of commutative rings.

With an additional assumption on $D$, one can go further. Let $H \subset G$ be a subgroup, and suppose that there is an $m>0$ for which the norm $N_{H}^{G}\left(i_{H}^{*} D\right)$ divides $D^{m}$. Write $D^{m}=D^{\prime} \cdot N_{H}^{G}\left(i_{H}^{*} D\right)$, and to keep the notation compact abbreviate $N_{H}^{G}\left(i_{H}^{*} D\right)$ to $N_{H}^{G} D$. Then there is a commutative diagram

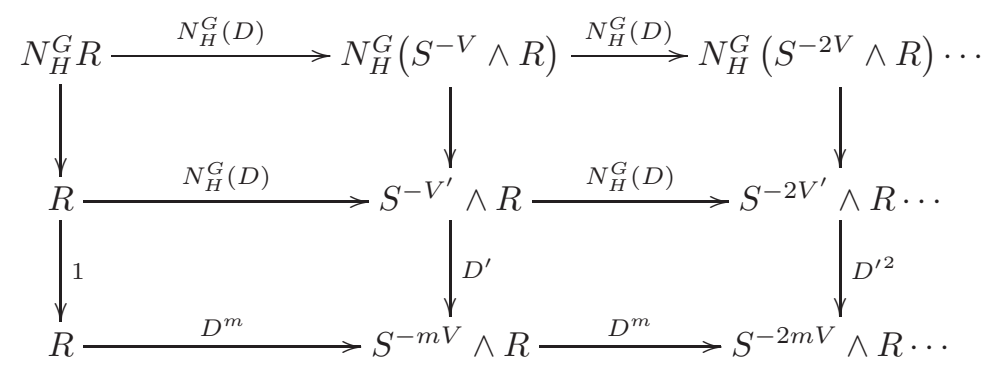

in which $V^{\prime}=\operatorname{ind}_{H}^{G} V$. Passing to the colimit gives a map

$$
N_{H}^{G} i_{H}^{*}\left(D^{-1} R\right) \rightarrow D^{-1} R
$$

extending the iterated multiplication. This allows one to form norms of elements in $\pi_{\star}^{H} D^{-1} R$ as if $D^{-1} R$ were an equivariant commutative ring.

A necessary condition for $D^{-1} R$ to actually be an equivariant commutative ring, is that for every $H \subset G$ the norm $N_{H}^{G} i_{H}^{*} D$ divides a power of $D$. In fact the condition is also sufficient. The proof of the result below is described in [29].

Proposition 9.15. Let $R$ be an equivariant commutative ring and $D \in \pi_{\star}^{G} R$. If $D$ has the property that for every $H \subset G$, the element $N_{H}^{G} i_{H}^{*} D$ divides a power of $D$, then the spectrum $D^{-1} R$ has a unique equivariant commutative algebra structure for which the map $R \rightarrow D^{-1} R$ is a map of commutative rings.

We will not make use of Proposition 9.15, as the ad hoc formation of norms from the non-trivial subgroups of $G$ is sufficient for our purpose.

Suppose that $t \in R O(G)$ and $u \in \pi_{t}^{H} D^{-1} R$ is represented at the $E_{2}$-term of the $R O(H)$-graded slice spectral sequence by the image of $u^{\prime} \in \pi_{t}^{H} H \mathbb{Z}$ under the map $\pi_{t}^{H} H \underline{\mathbb{Z}} \rightarrow \pi_{t}^{H} P_{0}^{0} D^{-1} R$ induced by the unit. We then have an $H$-equivariant commutative diagram

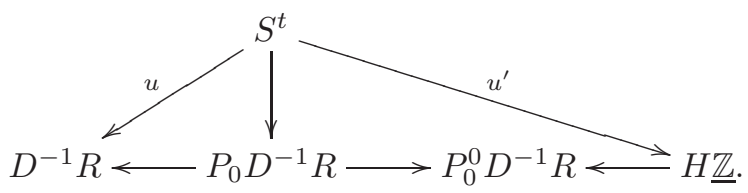

The maps in the bottom row are maps of homotopy commutative ring spectra. Since the formation of slice sections commutes with filtered colimits, if $N_{H}^{G} D$ divides a power of $D$ then the spectra along the bottom row also come equipped with maps $N_{H}^{G}(-) \rightarrow(-)$ extending the iterated multiplication, and compatible with the maps between them. This means we may apply the norm to the whole diagram to 
produce

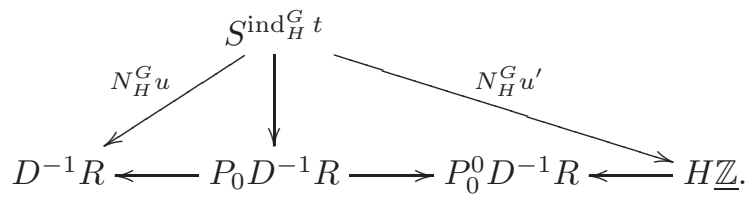

showing that $N_{H}^{G} u^{\prime}$ is a permanent cycle representing the class $N_{H}^{G} u \in \pi_{\text {ind }_{H}^{G}} D^{-1} R$.

We will take $R$ to be the spectrum $M U^{((G))}$. In order to specify the element $D$ we need to consider all of the spectra $M U^{((H))}$ for $H \subset G$, and we'll need to distinguish some of the important elements of the homotopy groups we've specified. We use (5.1) to map

$$
\pi_{\star}^{H} M U^{((H))} \rightarrow \pi_{\star}^{H} M U^{((G))},
$$

and make all of our computations in $\pi_{\star}^{H} M U^{((G))}$. Let

$$
\bar{r}_{i}^{H} \in \pi_{i \rho_{2}}^{C_{2}} M U^{((H))} \subset \pi_{i \rho_{2}}^{C_{2}} M U^{((G))}
$$

be the element defined in $\S 5.4 .2$, and

$$
\overline{\mathfrak{d}}_{k}^{H}=N_{C_{2}}^{H}\left(\bar{r}_{2^{k}-1}^{H}\right) \in \pi_{\left(2^{k}-1\right) \rho_{H}}^{H} M U^{((G))} .
$$

Finally, in addition to $g=|G|$ we will write $h=|H|$ for $H \subset G$.

Theorem 9.16. Let $D \in \pi_{\ell \rho_{G}}^{G} M U^{((G))}$ be any class having the property that for every nontrivial $H \subset G$, the element $N_{H}^{G} i_{H}^{*} D$ divides a power of $D$, and whose image in $\pi_{\star}^{H} M U^{((G))}$ is divisible by $\overline{\mathfrak{d}}_{g / h}^{H}$. The class $u_{2 \rho_{G}}^{2^{/ 2}}$ is a permanent cycle in the $R O(G)$-graded slice spectral sequence for $\pi_{\star}^{G} D^{-1} M U^{((G))}$.

Proof: By Corollary 9.13, for each nontrivial subgroup $H \subset G$, the class $\overline{\mathfrak{d}}_{g / h}^{H} u_{2 \sigma_{H}}^{2^{g / h}}$ is a permanent cycle in the $R O(H)$-graded slice spectral sequence for $\pi_{\star}^{H} M U^{((G))}$. Since $i_{H}^{*} D$ is divisible by $\overline{\mathfrak{d}}_{g / h}^{H}$, the class $u_{2 \sigma_{h}}^{2^{g / h}}$ is then a permanent cycle in the $R O(G)$-graded slice spectral sequence for $\pi_{\star}^{H} D^{-1} M U^{((G))}$. From this inventory of permanent cycles, and the ad hoc norm described above, we will show that $u_{2 \rho_{G}}^{g^{g / 2}}$ is also a permanent cycle.

To begin, note that if $H \subset G$ has index 2 , then $\operatorname{ind}_{H}^{G} 1=1+\sigma_{G}$. It follows from Lemma 3.13 that

$$
u_{2 \rho_{G}}=u_{2 \sigma_{G}}^{g / 2} N_{H}^{G} u_{2 \rho_{H}}
$$

This leads to the formula

$$
u_{2 \rho_{G}}^{k}=\prod_{0 \neq H \subset G} N_{H}^{G}\left(u_{2 \sigma_{H}}^{k h / 2}\right) .
$$

When $k=2^{g / 2}$ we have $k h / 2=2^{g / 2} h / 2 \geq 2^{g / h}$ for every $h \neq 1$ dividing $g$, so every term in the product is a permanent cycle (the inequality is an equality only when $h=2)$. This completes the proof.

Write $\Delta^{G}=u_{2 \rho_{G}} \overline{\mathfrak{d}}_{1}^{G}$.

Corollary 9.17. In the situation of Theorem 9.16 the class

$$
\left(\Delta^{G}\right)^{2^{g / 2}}=u_{2 \rho_{G}}^{2^{g / 2}}\left(\overline{\mathfrak{d}}_{1}^{G}\right)^{2 \cdot 2^{g / 2}}
$$


is a permanent cycle. Any class in $\pi_{2 \cdot g \cdot 2^{g / 2}}^{G} D^{-1} M U^{((G))}$ represented by (9.18) restricts to a unit in $\pi_{*}^{u} D^{-1} M U^{((G))}$.

Proof: The fact that (9.18) is a permanent cycle is immediate from Theorem 9.16. Since the slice tower refines the Postnikov tower, the restriction of an element in the $R O(G)$-graded group $\pi_{\star}^{G} D^{-1} M U^{((G))}$ to $\pi_{*}^{u} D^{-1} M U^{((G))}$ is determined entirely by any representative at the $E_{2}$-term of the slice spectral sequence. Since $u_{2 \rho_{G}}$ restricts to 1 , the restriction of any representative of (9.18) is equal to the restriction of $\left(\overline{\mathfrak{d}}_{1}^{G}\right)^{2 \cdot 2^{g / 2}}$, which is a unit since $\overline{\mathfrak{d}}_{1}^{G}$ divides $D$.

This gives

Theorem 9.19. With the notation of Theorem 9.16, if $M$ is any equivariant $D^{-1} M U^{((G))}$-module, then multiplication by $\left(\Delta^{G}\right)^{2^{g / 2}}$ is a weak equivalence

$$
\Sigma^{2 \cdot g \cdot 2^{g / 2}} i_{0}^{*} M \rightarrow i_{0}^{*} M
$$

and hence an isomorphism

$$
\left(\Delta^{G}\right)^{2^{g / 2}}: \pi_{*} M^{h G} \rightarrow \pi_{*+2 \cdot g \cdot 2^{g / 2}} M^{h G} .
$$

For example, in the case of $G=C_{2}$ the groups $\pi_{*}\left(D^{-1} M U^{((G))}\right)^{h G}$ are periodic with period $2 * 2 * 2=8$ and for $G=C_{4}$ there is a periodicity of $2 * 4 * 2^{2}=32$. For $G=C_{8}$ we have a period of $2 * 8 * 2^{4}=256$.

Remark 9.20. Suppose that $D \in \pi_{\star}^{G} R$ is of the form

$$
D=N_{C_{2}}^{G} x \text {. }
$$

Then for $C_{2} \subset H \subset G$ one has

$$
N_{H}^{G} i_{H}^{*} D=D^{g / h}
$$

Indeed,

$$
N_{H}^{G} i_{H}^{*} D=N_{H}^{G} i_{H}^{*} N_{C_{2}}^{G} x=N_{H}^{G}\left(N_{C_{2}}^{H}\right)^{g / h}=N_{C_{2}}^{G} x^{g / h}=D^{g / h} .
$$

Since each $\overline{\mathfrak{d}}_{k}^{H}$ has this form, any class $D$ which is a product of $N_{H}^{G} \overline{\mathfrak{d}}_{k}^{H}$ has the property required for Theorems 9.16 and 9.19 .

Corollary 9.21 (The Periodicity Theorem). Let $G=C_{8}$, and

$$
D=\left(N_{C_{2}}^{C_{8}} \overline{\mathfrak{d}}_{4}^{C_{2}}\right)\left(N_{C_{4}}^{C_{8}} \overline{\mathfrak{d}}_{2}^{C_{4}}\right)\left(\overline{\mathfrak{d}}_{1}^{C_{8}}\right) \in \pi_{19 \rho_{G}}^{G} M U^{((G))} .
$$

Then multiplication by $\left(\Delta^{G}\right)^{16}$ gives an isomorphism

$$
\pi_{*}\left(D^{-1} M U^{((G))}\right)^{h G} \rightarrow \pi_{*+256}\left(D^{-1} M U^{((G))}\right)^{h G} .
$$

Remark 9.22. For a periodicity theorem, one gets a sufficient inventory of powers of $u_{2 \sigma_{H}}$ as permanent cycles as long as for each $H$, some $\overline{\mathfrak{d}}_{j}^{H}$ is inverted. This is also enough to prove the Homotopy Fixed Point Theorem. Our particular choice of $\overline{\mathfrak{d}}_{g / h}^{H}$ is dictated by the requirements of the Detection Theorem. 


\section{The Homotopy Fixed Point Theorem}

Until now we haven't had occasion to refer to the function $G$-spectrum of maps from a pointed $G$-space $S$ to a $G$-spectrum $X$, which exists as part of the completeness of $\underline{\mathcal{S}}_{G}$ as a topological $G$-category. We will write $X^{S}$ for this object, so that

$$
\mathcal{S}^{G}\left(Z, X^{S}\right)=\mathcal{S}^{G}(Z \wedge S, X) .
$$

Definition 10.1. A $G$-spectrum $X$ is cofree if the map

$$
X \rightarrow X^{E G_{+}}
$$

adjoint to the projection map $E G_{+} \wedge X \rightarrow X$ is a weak equivalence.

If $X$ is cofree then the map

$$
\pi_{*}^{G} X \rightarrow \pi_{*}^{G} X^{E G_{+}}=\pi_{*} X^{h G}
$$

is an isomorphism. The main result of this section (Theorem 10.8) asserts that any module over $D^{-1} M U^{((G))}$ is cofree.

The map (10.2) is an equivalence of underlying spectra, and hence becomes an equivalence after smashing with any $G$-CW spectrum built entirely out of free $G$-cells. In particular, the map

$$
E G_{+} \wedge X \stackrel{\sim}{\longrightarrow} E G_{+} \wedge\left(X^{E G_{+}}\right)
$$

is an equivariant equivalence. One exploits this, as in [14], by making use of the pointed $G$-space $\tilde{E} G$ defined by the cofibration sequence

$$
E G_{+} \rightarrow S^{0} \rightarrow \tilde{E} G
$$

Lemma 10.5. For a $G$-spectrum $X$, the following are equivalent:

i) For all non-trivial $H \subset G$, the spectrum $\Phi^{H} X$ is contractible.

ii) The map $E G_{+} \wedge X \rightarrow X$ is a weak equivalence.

iii) The $G$-spectrum $\tilde{E} G \wedge X$ is contractible.

Proof: The equivalence of the second and third conditions is immediate from the cofibration sequence defining $\tilde{E} G$. Since $E G_{+}$is built from free $G$-cells, condition ii) implies condition i). For $H \subset G$ non-trivial, we have

$$
\Phi^{H}(\tilde{E} G \wedge X) \approx \Phi^{H}(\tilde{E} G) \wedge \Phi^{H}(X) \approx S^{0} \wedge \Phi^{H}(X) .
$$

Since the non-equivariant spectrum underlying $\tilde{E} G$ is contractible, condition i) therefore implies that $\Phi^{H}(\tilde{E} G \wedge X)$ is contractible for all $H \subset G$. But this means that $\tilde{E} G \wedge X$ is contractible (Proposition 2.52).

Corollary 10.6. If $R$ is an equivariant ring spectrum satisfying the equivalent conditions of Lemma 10.5 then any module over $R$ is cofree.

The condition of Corollary 10.6 requires $R$ to be an equivariant ring spectrum in the weakest possible sense, namely that $R$ possesses a unital multiplication (not necessarily associative) in ho $\mathcal{S}^{G}$. Similarly, the "module" condition is also one taking place in the homotopy category. 
Proof: Let $M$ be an $R$-module. Consider the diagram

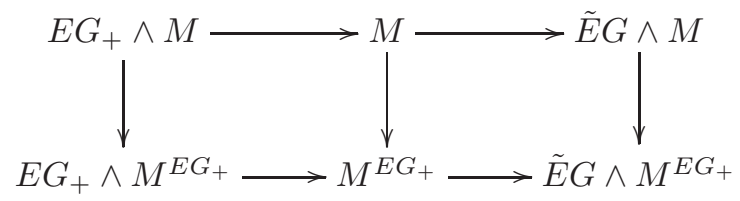

obtained by smashing $M \rightarrow M^{E G_{+}}$with the sequence (10.4). The fact that $R$ satisfies the condition i) of Lemma 10.5 implies that any $R$-module $M^{\prime}$ does since $\Phi^{H}(M)$ is a retract of $\Phi^{H}(R \wedge M) \approx \Phi^{H}(R) \wedge \Phi^{H}(M)$. Thus both $M$ and $M^{E G_{+}}$ satisfy the conditions of Lemma 10.5, and the terms on the right in (10.7) are contractible. The left vertical arrow is the weak equivalence (10.3). It follows that the middle vertical arrow is a weak equivalence.

Turning to our main purpose, we now consider a situation similar to the one in $\S 9.2$, and fix a class

$$
D \in \pi_{\ell \rho_{G}}^{G} M U^{((G))}
$$

with the property that for all non-trivial $H \subset G$ the restriction of $D$ to $\pi_{*}^{H} M U^{((G))}$ is divisible by $\overline{\mathfrak{d}}_{k}^{H}$ for some $k$ which may depend on $H$.

Theorem 10.8 (Homotopy Fixed Point Theorem). Any module $M$ over $D^{-1} M U^{((G))}$ is cofree, and so

$$
\pi_{*}^{G} M \rightarrow \pi_{*} M^{h G}
$$

is an isomorphism.

Proof: We will show that $D^{-1} M U^{((G))}$ satisfies condition i) of Lemma 10.5. The result will then follow from Corollary 10.6. Suppose that $H \subset G$ is non-trivial. Then

$$
\Phi^{H}\left(D^{-1} M U^{((G))}\right) \approx \Phi^{H}(D)^{-1} \Phi^{H}\left(M U^{((G))}\right) .
$$

But $D$ is divisible by $\overline{\mathfrak{d}}_{k}^{H}$, and so $\Phi^{H}(D)$ is divisible by

$$
\Phi^{H}\left(\overline{\mathfrak{d}}_{k}^{H}\right)=\Phi^{H}\left(N_{C_{2}}^{H}\left(\bar{r}_{2^{k}-1}^{H}\right)\right) y=\Phi^{C_{2}}\left(\bar{r}_{2^{k}-1}^{H}\right)
$$

which is zero by Proposition 5.50. This completes the proof.

Corollary 10.9. In the situation of Corollary 9.21, the map "multiplication by $\Delta^{G} "$ gives an isomorphism

$$
\pi_{*}^{G}\left(D^{-1} M U^{((G))}\right) \rightarrow \pi_{*+256}^{G}\left(D^{-1} M U^{((G))}\right) .
$$

Proof: In the diagram

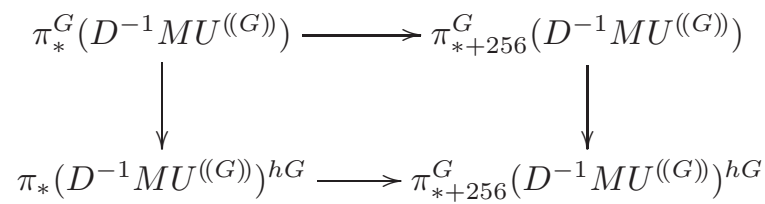

the vertical maps are isomorphisms by Theorem 10.8, and the bottom horizontal map is an isomorphism by Corollary 9.21 . 


\section{The Detection Theorem}

11.1. Outline of the proof. We now turn to the proof of the Detection Theorem. For the convenience of the reader, we restate the result.

Theorem 11.1 (The Detection Theorem). If $\theta_{j} \in \pi_{2^{j+1}-2} S^{0}$ is an element of Kervaire invariant 1 , and $j>2$, then the image of $\theta_{j}$ in $\pi_{2^{j+1}-2} \Omega$ is non-zero.

To recapitulate, we are working with the group $G=C_{8}$, and the spectrum $\Omega$ is the spectrum of $G$-fixed points in $\Omega_{\mathbb{O}}=D^{-1} M U^{((G))}$, with $D \in \pi_{19 \rho} M U^{((G))}$ the element specified in Corollary 9.21.

Theorem 11.2 (Algebraic Detection Theorem). If

$$
x \in \operatorname{Ext}_{M U_{*}(M U)}^{2,2^{j+1}}\left(M U_{*}, M U_{*}\right)
$$

is any element mapping to

$$
h_{j}^{2} \in \operatorname{Ext}_{\mathcal{A}}^{2,2^{j+1}}(\mathbb{Z} / 2, \mathbb{Z} / 2)
$$

in the $E_{2}$-term of the classical Adams spectral sequence, and $j>2$, then the image of $x$ in $H^{2}\left(C_{8} ; \pi_{2^{j+1}}^{u} \Omega_{\mathbb{O}}\right)$ is nonzero.

We will prove the Algebraic Detection Theorem by establishing the following.

Proposition 11.3. For $j>2$, there is a map

$$
H^{2}\left(C_{8} ; \pi_{2^{j+1}} \Omega_{\mathbb{O}}\right) \rightarrow \mathbb{Q} / \mathbb{Z}
$$

making the diagram

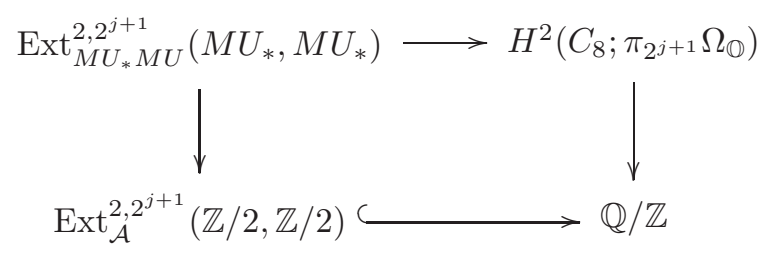

commute.

In (11.5), the bottom row is the Kervaire invariant homomorphism sending $h_{j}^{2}$ to $1 / 2$. Since the vector space

$$
\operatorname{Ext}_{\mathcal{A}}^{2,2^{j+1}}(\mathbb{Z} / 2, \mathbb{Z} / 2)
$$

has dimension 1, with basis $h_{j}^{2}$ (Adams [3, Theorem 2.5.1]), the Kervaire invariant homomorphism is completely specified by this property, and is a monomorphism. In plain language, Proposition 11.3 asserts that the Kervaire invariant homomorphism, thought of as a map

$$
\operatorname{Ext}_{M U_{*} M U}^{2,2^{j+1}}\left(M U_{*}, M U_{*}\right) \rightarrow \mathbb{Q} / \mathbb{Z}
$$

factors through $H^{2}\left(C_{8}, \pi_{2^{j+1}} \Omega_{\mathbb{O}}\right)$. This directly implies Theorem 11.2.

Remark 11.6. All three of these results (Theorems 11.1 and 11.2, and Proposition 11.3) remain true without the restriction $j>2$. The other cases $j \leq 2$ require separate arguments, and are not needed for the proof of Theorem 1.1, so we do not include them. 
We now describe the proof of Proposition 11.3, deferring the details to later subsections. In order to construct the map (11.4) we use of the theory of formal $A$-modules to construct a $C_{8}$-equivariant ring homomorphism from $\pi_{*}^{u} \Omega_{\mathbb{O}}$ to much smaller ring. Let $A=\mathbb{Z}_{2}[\zeta]$ be the 2-adic completion of the ring obtained by adjoining an $8^{\text {th }}$ root of unity to the ring of integers, and $F_{f}$ the Lubin-Tate formal $A$-module over $A$ associated to any choice of power series $f(x) \in A[x]$ satisfying (see $\S 11.2$ )

$$
\begin{aligned}
& f(x) \equiv \pi x \quad \bmod \left(x^{2}\right) \\
& f(x) \equiv x^{2} \quad \bmod (\pi),
\end{aligned}
$$

with uniformizer $\pi=\zeta-1$. By construction, there is an isomorphism

$$
\begin{aligned}
A \stackrel{\approx}{\rightarrow} \operatorname{End}\left(F_{f}\right) \\
a \mapsto[a](x)
\end{aligned}
$$

satisfying $[a]^{\prime}(0)=a$. Using the map $\gamma \mapsto \zeta$ to identify the group of $8^{\text {th }}$ roots of unity with $C_{8}$ gives an action of $C_{8}$ on $F_{f}$ extending the canonical action of $C_{2}$ by formal multiplication by -1 . As described in $\$ 11.3 .4$ below, this data is classify by a $C_{8}$-equivariant map of graded rings

$$
\pi_{*}^{u} M U^{\left(\left(C_{8}\right)\right)} \rightarrow A_{*}
$$

in which $A_{*}=A\left[u^{ \pm 1}\right],|u|=2$, and in which the action of the chosen generator $\gamma \in C_{8}$ is the $A$-algebra map sending $u$ to $\zeta u$. The first thing to check about this map is

Proposition 11.8. The image of $D \in \pi_{19 \rho} M U^{\left(\left(C_{8}\right)\right)}$ under

$$
\pi_{19 \rho} M U^{\left(\left(C_{8}\right)\right)} \rightarrow \pi_{152}^{u} M U^{\left(\left(C_{8}\right)\right)} \rightarrow A_{152}
$$

is a unit, hence (11.7) factors uniquely through a $C_{8}$-equivariant map

$$
\pi_{*}^{u} \Omega_{\mathbb{O}} \rightarrow A_{*} .
$$

Let

$$
\chi: H^{2}\left(C_{8} ; \pi_{2^{j+1}}^{u} \Omega_{\mathbb{O}}\right) \rightarrow H^{2}\left(C_{8} ; A_{2^{j+1}}\right)
$$

be the map of cohomology groups induced by (11.9). Using $\chi$, form the rightmost arrow in the diagram below

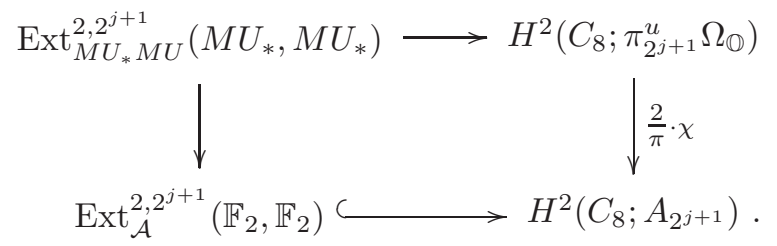

For the bottom arrow, note that both $\operatorname{Ext}_{\mathcal{A}}^{2,2^{j+1}}(\mathbb{Z} / 2, \mathbb{Z} / 2)$ and $H^{1}\left(C_{8} ; A_{2^{j+1}} /(\pi)\right)$ are cyclic of order 2 , and hence isomorphic by a unique isomorphism. The bottom arrow in (11.10) is defined to be the map corresponding to the connecting homomorphism

$$
H^{1}\left(C_{8} ; A_{2^{j+1}} /(\pi)\right) \rightarrow H^{2}\left(C_{8} ; A_{2^{j+1}}\right)
$$


under this isomorphism. For $j>2$, the action of $\gamma$ on $u^{2^{j}}$ is trivial, and so $H^{2}\left(C_{8}, A_{2^{j+1}}\right) \approx A_{2^{j+1}} /(8)$, and one easily checks that this map is a monomorphism.

The main point is the commutativity of the diagram. Once that is established, the map (11.4) can be taken to be the composition of the right vertical arrow in (11.10) with any map (dashed arrow)

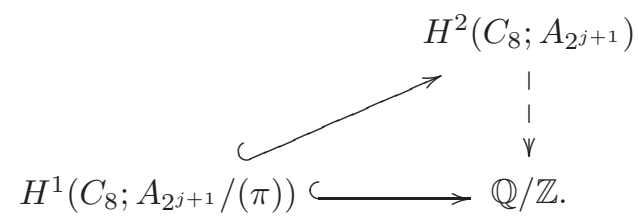

factoring the inclusion through the connecting homomorphism.

Checking the commutativity of (11.10) involves some technical details about the groups $\operatorname{Ext}_{M U_{*} M U}^{2,2^{j+1}}\left(M U_{*}, M U_{*}\right)$. The following lemma can be read off from [78, Theorem 1.5] (see $\S 11.6)$.

Lemma 11.11. For $j>1$, the map

$$
\operatorname{Ext}_{M U_{*} M U}^{1,2^{j+1}}\left(M U_{*}, M U_{*} /(2)\right) \rightarrow \operatorname{Ext}_{M U_{*} M U}^{2,2^{j+1}}\left(M U_{*}, M U_{*}\right)
$$

is surjective after localizing at 2 .

Lemma 11.11 enables us to replace the upper left corner of (11.10) with the

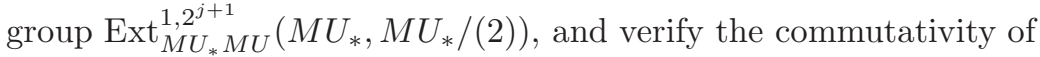

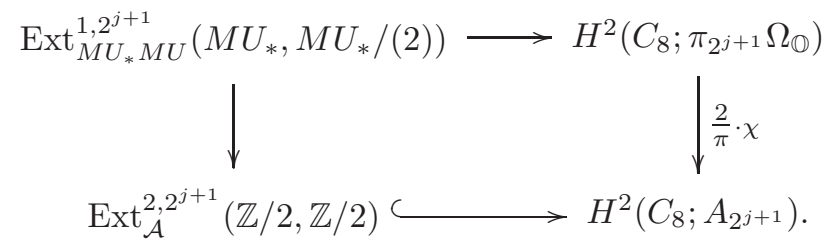

The key technical point in doing this is

Proposition 11.13. The maps

$$
\begin{gathered}
\operatorname{Ext}_{M U_{*} M U}^{1,2^{j+1}}\left(M U_{*}, M U_{*} /(2)\right) \rightarrow \operatorname{Ext}_{\mathcal{A}}^{2,2^{j+1}}(\mathbb{Z} / 2, \mathbb{Z} / 2) \\
\operatorname{Ext}_{M U_{*} M U}^{1,2^{j+1}}\left(M U_{*}, M U_{*} /(2)\right) \rightarrow H^{1}\left(C_{8} ; A_{2^{j+1}} /(\pi)\right)
\end{gathered}
$$

are surjective and have the same kernel.

Proposition 11.13 gives the commutativity of the left square in

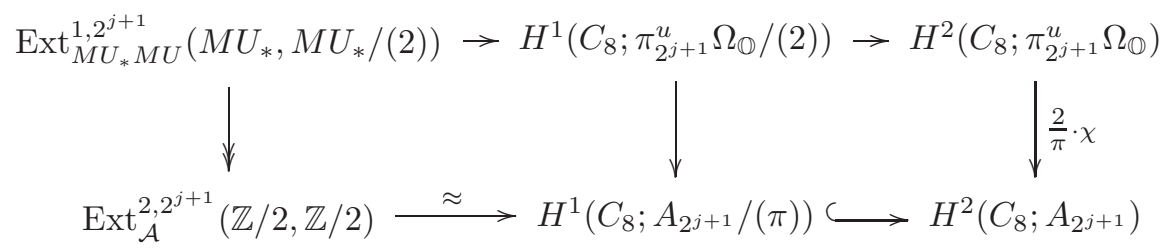

The commutativity of the right hand square follows from the naturality of the connecting homomorphism. The outer square is (11.12). This completes our summary of the proof of the Proposition 11.3 and the Detection Theorem. 
Remark 11.14. The argument of this section can be easily adapted to prove a detection theorem for $M U^{\left(\left(C_{2} n\right)\right)}$ as long as $n \geq 3$. The result does not hold in the cases $n<3$. What fails is the assertion in Proposition 11.13 that the two maps have the same kernel. This assertion makes essential use of the fact that the reduction of the Lubin-Tate group over $A /(\pi)$ has height greater than 2 .

The remainder of this section is devoted to filling in the details of this outline. We begin in $\S 11.2$ by recalling the Lubin-Tate formal $A$-module [49] and some simple but useful results relating the power series $[a](x)$ to the $\pi$-adic valuation of $a$. We turn in $\S 11.3$ to the ideas connecting the Adams-Novikov $E_{2}$-term to group cohomology. In $\S 11.3 .4$ we describe the "conjugation action" and prove Proposition 11.28 which describes the functor co-represented by $\pi_{*}^{u} M U^{((G))}$ on the category of $G$-equivariant graded commutative rings. Setting all of this up brings us as far as the statement of Proposition 11.8 which is proved in $\$ 11.4$. Proposition 11.13 is proved in $\S 11.5$. The proof relies heavily on the computations in [63] and [78], in the form of Proposition 11.34. An addendum to this section discusses how these computations are made, and how they lead to Lemmas 11.11 and Proposition 11.34.

The reader may also wish to consult [64] for another presentation of these ideas.

11.2. Formal $A$-modules and the Lubin-Tate group. Let $A$ and $R$ be commutative rings, and $e: A \rightarrow R$ a ring homomorphism. A (1-dimensional) formal $A$-module over $R$ is a formal group law $F$ over $R$, equipped with a ring homomorphism

$$
\begin{aligned}
A & \rightarrow \operatorname{End}(F) \\
a & \mapsto[a](x)
\end{aligned}
$$

with the property that $[a]^{\prime}(0)=e(a)$. In the case of interest to us, $e$ is a monomorphism (in fact the identity map), and we will not distinguish in notation between $a$ and $e(a)$.

Formal $A$-modules were introduced by Lubin and Tate in their work [49] on local class field theory. For $A$ the ring of integers in a local field with finite residue field, they constructed a formal $A$-module over $A$ itself, unique up to isomorphism. Their construction starts with a choice of uniformizer $\pi \in A$ and a power series

$$
f(x) \in A[[x]]
$$

intended to be the endomorphism $[\pi](x)$. Writing $q$ for the order of the residue field, the power series $f$ is required to satisfy

$$
\begin{aligned}
& f(x) \equiv \pi x \quad \bmod \left(x^{2}\right) \\
& f(x) \equiv x^{q} \quad \bmod (\pi) .
\end{aligned}
$$

For example, $f(x)$ could be taken to be $\pi x+x^{q}$. Lubin and Tate showed that such an $f$ determines a formal $A$-module in which the formal sum is the unique power series $F_{f}(x, y) \in A[[x]]$ satisfying

$$
\begin{aligned}
F_{f}(x, y) & \equiv x+y \quad \bmod (x, y)^{2} \\
F_{f}(f(x), f(y)) & =f\left(F_{f}(x, y)\right),
\end{aligned}
$$

and for $a \in A$ the power series $[a](x)$ is the unique power series satisfying

$$
\begin{aligned}
{[a](x) } & \equiv a x \quad \bmod (x)^{2} \\
{[a](f(x)) } & =f([a](x)) .
\end{aligned}
$$


In particular, one does indeed have $[\pi](x)=f(x)$.

Continuing with the Lubin-Tate formal $A$-module, for $a \in A$ write

$$
[a](x) \equiv \alpha_{d} x^{d}+\cdots \quad \bmod (\pi)
$$

with $0 \neq \alpha_{d} \in A /(\pi)$. One easily checks that the function $\nu(a)=\log _{q}(d)$ defines a valuation on $A$. The the fact that $[\pi](x)=f(x)$ implies that $\nu$ is the unique valuation for which $\nu(\pi)=1$.

We are interested in the case

$$
A=\mathbb{Z}_{2}[\zeta]
$$

with $\pi=\zeta-1$, and any fixed choice of $f(x)$. Since $\nu(\zeta-1)=1, \nu\left(\zeta^{2}-1\right)=2$ and $\nu\left(\zeta^{4}-1\right)=4$, and since any unit in $A$ is congruent to 1 modulo $\pi$, this means that modulo $(\pi)$

$$
\begin{aligned}
{[\zeta-1](x) } & \equiv x^{2}+\cdots \\
{\left[\zeta^{2}-1\right](x) } & \equiv x^{4}+\cdots \\
{\left[\zeta^{4}-1\right](x) } & \equiv x^{16}+\cdots,
\end{aligned}
$$

and so

$$
\begin{aligned}
{[\zeta](x) } & \equiv x \underset{F_{f}}{+} x^{2}+\cdots \\
& \equiv x+x^{2}+\cdots \\
{\left[\zeta^{2}\right](x) } & \equiv x \underset{F_{f}}{+} x^{4}+\cdots \\
& \equiv x+x^{4}+\cdots \\
{\left[\zeta^{4}\right](x) } & \equiv x \underset{F_{f}}{+} x^{16}+\cdots \\
& \equiv x+x^{16}+\cdots
\end{aligned}
$$

These congruences play an important role in the proof of Proposition 11.8.

Remark 11.16. The formulae (11.15) are independent of the choice of $f(x)$. In particular they hold for a choice of $f$ leading to a 2-typical formal group law.

11.3. Group actions and homogeneous formal group laws. We now turn to the relationship between group actions on formal group laws and group cohomology. Our eventual goal involves some explicit formulas, so we begin with a relatively detailed summary.

11.3.1. Homogeneous formal group laws. Suppose that $R_{*}=\bigoplus R_{n}$ is a graded commutative ring. By Quillen's work $[67,4]$ the set of graded ring homomorphisms

$$
M U_{*} \rightarrow R_{*}
$$

is in one to one correspondence with the set of formal group laws $F$ over $R$ which are homogeneous of degree -2 in the sense that the formal sum

$$
F(x, y)
$$

is homogeneous of degree -2 , when $x$ and $y$ are given degree -2 . In terms of the power series

$$
F(x, y)=x+y+\sum a_{i j} x^{i} y^{j}
$$

this means that $a_{i j}$ has degree $2(i+j-1)$. 
The graded ring $M U_{*} M U=\pi_{*} M U \wedge M U$ co-represents the functor associating to a graded ring $R_{*}$ the set of pairs $\left(F_{1}, F_{2}\right)$ of homogeneous formal group laws and an isomorphism $g: F_{1} \rightarrow F_{2}$ between them which is strict in the sense that it is given by a power series of the form

$$
[g](x)=x+O\left(x^{2}\right) .
$$

More generally, the ring $\pi_{*} M U^{(n)}$ ( $n$-fold smash product) co-represents functor associating to a graded ring $R_{*}$ the set of chains

$$
F_{1} \rightarrow \cdots \rightarrow F_{n}
$$

of homogeneous formal group laws and strict isomorphisms over $R$. The standard convention is that the homogeneous formal group law $F_{i}$ is the one classified by the map

$$
\pi_{*} M U \rightarrow \pi_{*} M U \wedge \cdots \wedge M U
$$

induced from the inclusion of the $i^{\text {th }}$ factor

$$
S^{0} \wedge \cdots \wedge M U \wedge \cdots \wedge S^{0} \rightarrow M U \wedge \cdots \wedge M U .
$$

Taken together, the pair $\left(M U_{*}, M U_{*} M U\right)$ forms the Hopf algebroid which corepresents the functor associating to a graded commutative ring $R_{*}$ the groupoid of homogeneous formal group laws over $R_{*}$ and strict isomorphisms. For the definition of Hopf algebroid, the reader is referred to [71, Definition A1.1.1].

11.3.2. Group actions. Let $\mathcal{M}_{F G}$ be the category of pairs $(R, F)$, with $F$ a formal group law over a commutative ring $R$, and in which a morphism

$$
(f, \psi):\left(R_{1}, F_{1}\right) \rightarrow\left(R_{2}, F_{2}\right)
$$

consists of a ring homomorphism $f: R_{1} \rightarrow R_{2}$, and an isomorphism of formal group laws $\psi: F_{2} \stackrel{\approx}{\rightarrow} f^{*} F_{1}$. Morphisms can also be described as ring homomorphisms

$$
\begin{aligned}
h: R_{1}[[x]] & \rightarrow R_{2}[\llbracket x] \\
h(r) & =f(r) \\
h(x) & =\psi(x)
\end{aligned}
$$

which are compatible with the formal sum in the sense that the diagram

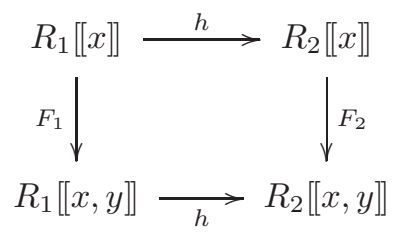

commutes. Let $\mathcal{M}_{F G}^{h}$ be the analogous category of homogeneous formal group laws over graded rings, and strict isomorphisms.

The categories $\mathcal{M}_{F G}$ and $\mathcal{M}_{F G}^{h}$ are related by the strictification functor

$$
\begin{aligned}
\mathcal{M}_{F G} & \rightarrow \mathcal{M}_{F G}^{h} \\
(R, F) & \mapsto\left(R_{*}, F^{h}\right) .
\end{aligned}
$$

The ring $R_{*}=R[u]$ is obtained from $R$ by adjoining a polynomial variable $u$ with $|u|=2$, and $F^{h}$ is the unique formal power series satisfying

$$
u F^{h}(x, y)=F(u x, u x) .
$$


The strictification of a map $(f, \psi):(R, F) \rightarrow\left(R^{\prime}, F^{\prime}\right)$ is the pair

$$
\left(f^{h}, \psi^{h}\right):\left(R_{*}, F^{h}\right) \rightarrow\left(R_{*}^{\prime},\left(F^{\prime}\right)^{h}\right)
$$

with $f^{h}(u)=\psi^{\prime}(0) u$ and $\psi^{h}(x)$ the unique power series satisfying $u \psi^{h}(x)=\psi(u x)$.

A (left) action of a group $G$ on a pair $(R, F) \in \mathcal{M}_{F G}$ is a map of monoids

$$
G \rightarrow \mathcal{M}_{F G}((R, F),(R, F))
$$

and a strict (left) action of a group on $\left(R_{*}, F\right) \in \mathcal{M}_{F G}^{h}$ is a map

$$
G \rightarrow \mathcal{M}_{F G}^{h}\left(\left(R_{*}, F\right),\left(R_{*}, F\right)\right) .
$$

The strictification functor converts a group action into a strict one.

A left action of $G$ on $(R, F)$ corresponds to a left action of $G$ on $R[x]$. We will use the notation

$$
\begin{aligned}
& r \mapsto g r \quad r \in R \\
& x \mapsto[g](x)
\end{aligned}
$$

for this action.

Example 11.18. Suppose that $E$ is a complex oriented, homotopy commutative ring spectrum, and that a finite group $G$ acts on $E$ by homotopy multiplicative maps. Let $F$ denote the corresponding homogeneous formal group law over $\pi_{*} E$. Then the action of $G$ on $E^{*}\left(\mathbf{C P}^{\infty}\right)$ gives a strict action of $G$ on $\left(\pi_{*} E, F\right)$.

Example 11.19. The group $C_{2}$ acts on any $(R, F) \in \mathcal{M}_{F G}$ as the identity map on $R$ and formal multiplication by $(-1)$ on $F$.

11.3.3. Group cohomology and the Adams-Novikov spectral sequence. When $\left(R_{*}, F\right)$ is a homogeneous formal group law equipped with a strict action of a group $G$, there is a map

$$
\operatorname{Ext}_{M U_{*} M U}^{s, t}\left(M U_{*}, M U_{*}\right) \rightarrow H^{s}\left(G ; R_{t}\right)
$$

Conceptually, it arises from the inclusion functor of the subcategory of $\mathcal{M}_{F G}^{h}$ whose only object is $\left(R_{*}, F\right)$ and whose monoid of self maps is given by the action of $G$. For the purposes of explicit computation, it is conveniently described as derived from a map of Hopf algebroids

$$
\left(M U_{*}, M U_{*} M U\right) \rightarrow\left(R_{*}, C\left(G ; R_{*}\right)\right),
$$

in which $C\left(G ; R_{*}\right)$ is the ring of (set-theoretic) functions from $G$ to $R_{*}$.

The Hopf algebroid $\left(R_{*}, C\left(G ; R_{*}\right)\right)$ is the one expressing the group action of $G$ on $R_{*}$. The "source" map

$$
\eta_{L}: R_{*} \rightarrow C\left(G ; R_{*}\right)
$$

sends $r \in R_{*}$ to the constant function with value $r$, and the "target" map $\eta_{R}$ : $R_{*} \rightarrow C\left(G ; R_{*}\right)$ is the transpose of the action mapping. It associates to $r \in R_{*}$ the function sending $g \in G$ to $g \cdot r$. The coproduct

$$
\Delta: C\left(G ; R_{*}\right) \rightarrow C\left(G ; R_{*}\right) \underset{R_{*}}{\otimes} C\left(G ; R_{*}\right)
$$

is the composition of the map

$$
C\left(G ; R_{*}\right) \rightarrow C\left(G \times G, R_{*}\right)
$$

dual to multiplication in $G$, and the isomorphism

$$
C\left(G ; R_{*}\right) \underset{R_{*}}{\otimes} C\left(G ; R_{*}\right) \stackrel{\approx}{\rightarrow} C\left(G \times G, R_{*}\right)
$$


given by setting

$$
\left(f_{1} \otimes f_{2}\right)\left(g_{1}, g_{2}\right)=f_{1}\left(g_{1}\right) \cdot g_{1} f_{2}\left(g_{2}\right) .
$$

The map (11.20) consists of the map $M U_{*} \rightarrow R_{*}$ classifying the homogeneous formal group law $F$, and the map $M U_{*} M U \rightarrow C\left(G, R_{*}\right)$, defined by declaring the composition

$$
M U_{*} M U \rightarrow C\left(G, R_{*}\right) \stackrel{\mathrm{ev}_{g}}{\longrightarrow} R_{*}
$$

to be the map classifying the strict isomorphism

$$
[g](x): F \rightarrow g^{*} F .
$$

When the $G$-action on $\left(R_{*}, F\right)$ arises, as in Example 11.18, from an action of $G$ on a complex oriented homotopy commutative ring spectrum $E$, the map (11.20) is the $E_{2}$-term of a map of spectral sequences abutting to the homomorphism $\pi_{*} S^{0} \rightarrow \pi_{*} E^{h G}$. We couldn't quite find this result in the literature (though [16, Proposition 6.7] is close). To see it, let

$$
C^{\bullet}(G ; E)=\operatorname{Map}^{G}\left(E G_{\bullet}, E\right)
$$

be the cosimplicial spectrum of $G$-maps from the bar construction model for $E G$. into $E$. Thus

$$
C^{n}(G ; E)=\prod_{G^{n}} E
$$

and $\operatorname{Tot} C^{\bullet}(G ; E)$ is the homotopy fixed point spectrum $E^{h G}$. The cosimplical ring $[n] \mapsto \pi_{*} C\left(G^{n}, E\right)$ is the nerve of the Hopf-algebroid $\left(\pi_{*} E, C\left(G, \pi_{*} E\right)\right)$ and forms the cobar complex for calculating $H^{*}\left(G, \pi_{*} E\right)$. The homotopy fixed point spectral sequence is the homotopy spectral sequence of this cosimplicial spectrum.

Choose a complex orientation for $E$, and for every $n \geq 0$ let

$$
M U^{(n+1)} \rightarrow C^{n}(G ; E) \in \operatorname{ho} \mathcal{S}
$$

be a representative of the unique homotopy class of homotopy multiplicative maps whose restriction to the $i^{\text {th }}$ smash factor of $M U^{(n+1)}$ is the composition of the complex orientation

$$
M U \rightarrow E=C^{0}(G ; E)
$$

with the cosimplicial structure map $C^{0}(G ; E) \rightarrow C^{n}(G ; E)$ corresponding to the inclusion of the $i^{\text {th }}$-vertex of $\Delta[n]$. The maps (11.21) fit into a homotopy commutative map of cosimplicial resolutions

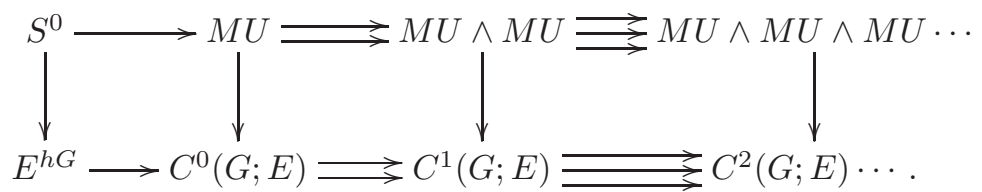

If this were an actually commutative diagram, the desired spectral sequence would be the one derived from the induced map of "Tot towers." Even though it is only homotopy commutative, the fact that the top row is an $M U$ Adams resolution and the spectra in the bottom row are complex oriented means that it can be still be refined to a map of towers.

This result doesn't quite seem to appear in the literature, though an assertion along these lines is made in [61, pp 289-90], and the case in which $M U$ is replaced by $E_{n}$ is [16, Proposition 6.2]. To spell out the details, we begin with some generalities 
about the Tot tower of a cosimplical spectrum $X^{\bullet}$. Let $N X^{n}$ be the iterated mapping cone of the coface maps

$$
d^{i}: X^{n-1} \rightarrow X^{n}, \quad i=1, \ldots, n .
$$

The spectrum $N X^{n}$ is a retract of $X^{n}$ (it is split by the inclusion of the iterated homotopy fiber of the co-degeneracy maps). By construction it depends, as a retract of $X^{n}$, functorially on $X^{\bullet}$. The spectra $N X^{n}$ fit into a sequence

$$
N X^{0} \stackrel{d^{0}}{\rightarrow} N X^{1} \stackrel{d^{0}}{\rightarrow} \cdots
$$

which is a "complex" in the sense that the composition of any two maps is null homotopic.

The homotopy spectral sequence of the cosimplicial spectrum $X^{\bullet}$ is derived from the tower $\left\{\operatorname{Tot}_{n} X^{\bullet}\right\}$. For our purposes it is easier to work with the fibers of the map from Tot $X^{\bullet}$. Write $F X^{0}=\operatorname{Tot} X^{\bullet}$, and define $F X^{n}$ to be the homotopy fiber of the map Tot $X^{\bullet} \rightarrow \operatorname{Tot}_{n-1} X^{\bullet}$. Then there is a functorial fibration sequence

$$
F X^{n} \rightarrow F X^{n-1} \rightarrow \Sigma^{-(n-1)} N X^{(n-1)} \text {. }
$$

Of course the homotopy spectral sequence can also be derived from the tower $\left\{F X^{n}\right\}$, for example by using it to reconstruct the Tot-tower.

To simplify the notation, write $X^{\bullet}=M U^{\bullet+1}$ and $Y^{\bullet}=C^{\bullet}(G ; E)$ for the cosimplicial spectra occurring in the top and bottom rows of (11.22). The complex $N X^{\bullet}$ is the standard $M U$-Adams resolution for $S^{0}$, and in this case the fibration sequence (11.23) is equivalent to

$$
F X^{n} \rightarrow F X^{n-1} \rightarrow M U \wedge F X^{n-1} .
$$

The consequence we need of this is the characterizing property of an Adams tower: if $R$ is any $M U$-module, then the connecting homomorphism

$$
\left[F X^{n}, R\right] \hookrightarrow\left[\Sigma^{-n-1} N X^{n}, R\right]
$$

is a monomorphism.

Our aim is to construct a map of towers

$$
\left\{F X^{n}\right\} \rightarrow\left\{F Y^{n}\right\} .
$$

Suppose by induction we have produced a homotopy commutative diagram

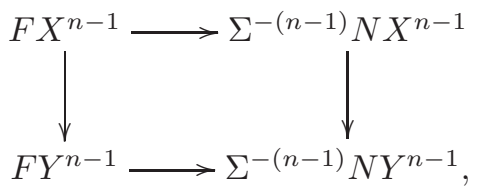

and choose any map $F X^{n} \rightarrow F Y^{n}$ making

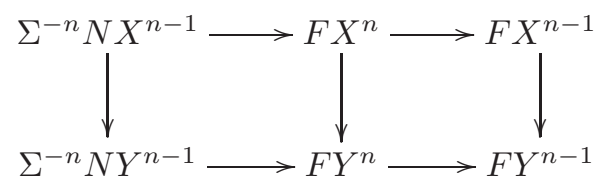


commute up to homotopy. We claim that the diagram

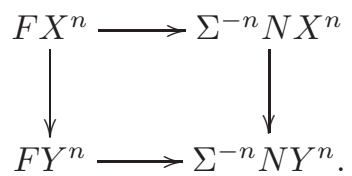

also commutes up to homotopy. The claim completes the induction step, and gives (11.25).

To verify the claim, consider

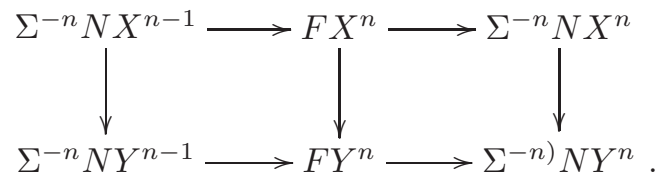

The outermost square commutes since it is a retract of a suspension of one of the squares in (11.22). The spectrum $\Sigma^{-(n+1)} N Y^{n+1}$ admits the structure of an $M U$-module since it is a retract of a suspension of $C^{n+1}(G ; E)$ which is complex orientable. Taking it for $R$ in the monomorphism (11.24) shows that the commutativity of the outer square in (11.26) implies the commutativity of the right hand square. This verifies the claim.

11.3.4. The conjugation action. Applying the strictification functor to Example 11.19, one is led to the "conjugation action" of $C_{2}$ on homogeneous formal group laws over graded rings.

Let $F$ be a homogeneous formal group law over a graded commutative ring $R_{*}$, and $c: R_{*} \rightarrow R_{*}$ any ring homomorphism with the property that $c: R_{2 n} \rightarrow R_{2 n}$ the map given by multiplication by $(-1)^{n}$. The homogeneous formal group law $F^{c}:=c^{*} F$ is given by

$$
F^{c}(x, y)=-F(-x,-y)
$$

The power series

$$
c(x)=-[-1]_{F}(x)
$$

has the property that $c \circ c(x)=x$ and provides both a strict isomorphism $F \rightarrow F_{c}$ and its inverse $F^{c} \rightarrow F$. These combine to give an action of $C_{2}$ on $\left(R_{*}, F\right)$ which we call the conjugation action associated to $c: R_{*} \rightarrow R_{*}$.

The map $c$ is completely specified on the even degree elements in $R_{*}$ and in general there are as many conjugation actions as there are ways of extending $c$ to all of $R_{*}$. In the examples of interest to us, $R_{*}$ will be evenly graded, and so there is exactly one conjugation action.

Example 11.27. If $E$ is a real-oriented spectrum, then the underlying $C_{2}$ action on $\left(i_{0}^{*} E\right)^{*}\left[\left[\mathbf{C P}^{\infty}\right]\right]$ is the conjugation action. The case $E=M U_{\mathbb{R}}$ is universal in the sense that the map $M U_{*} \rightarrow R_{*}$ classifying a homogeneous formal group law is equivariant for any choice of conjugation action.

We now generalize Example 11.27. Let $G=C_{2^{n}}$, and give $i_{1}^{*} M U^{((G))}$ the real orientation coming from the unit

$$
M U_{\mathbb{R}} \rightarrow i_{1}^{*} M U^{((G))}
$$


of the norm-restriction adjunction on equivariant commutative algebras (Example 5.6). Examples 11.18 and 11.27 then equip $\left(\pi_{*}^{u} M U^{((G))}, F\right)$ with a $G$-action extending the conjugation action of $C_{2}$.

Proposition 11.28. The pair $\left(\pi_{*}^{u} M U^{((G))}, F\right)$ equipped with its $G$-action is universal in the sense that map associating to a $G$-equivariant

$$
f: \pi_{*}^{u} M U^{((G))} \rightarrow R_{*},
$$

the pair $\left(R *, f^{*} F\right)$ with its induced $G$-action, is a bijection between the set of equivariant maps (11.29) and the set of $\left(R_{*}, F\right)$ equipped with a $G$-action extending the one on $R_{*}$, and the conjugation action of $C_{2} \subset G$.

Proof: Suppose that $\left(R_{*}, F\right)$ is a homogeneous formal group law over a graded ring, equipped with a $G$-action extending the conjugation action of $C_{2}$. Choose a generator $\gamma \in G$. This data is equivalent to an isomorphism

$$
\tau: F_{1} \rightarrow F_{0}
$$

having the property that the composite of the chain of isomorphisms

$$
\begin{gathered}
F_{g / 2} \stackrel{\tau_{g / 2-1}}{\longrightarrow} F_{g / 2-1} \rightarrow \cdots \stackrel{\tau_{1}}{\longrightarrow} F_{1} \stackrel{\tau_{0}}{\longrightarrow} F_{0} \\
\tau_{i}=\left(\gamma^{i}\right)^{*} \tau
\end{gathered}
$$

is the conjugation isomorphism $c$. The claim then follows from the decomposition (5.2) and the description of $\pi_{*} M U \wedge \cdots \wedge M U$ in terms of chains of composable strict isomorphisms.

11.4. The fundamental representation. As described in $\S 11.1$ these ideas can be used to construct a $C_{8}$-equivariant ring homomorphism from $\pi_{*}^{u} \Omega_{\mathbb{O}}$ to much smaller ring. Let $A=\mathbb{Z}_{2}[\zeta]$ be as in $\S 11$, and $F_{f}$ the Lubin-Tate formal $A$-module over $A$ associated to a power series series $f(x)$ leading to a 2-typical formal group law. Using $\zeta$ to identify the group of $8^{\text {th }}$-roots of unity with $C_{8}$, we get a $C_{8}$ action on $\left(A, F_{f}\right)$. From this apply the strictification functor to get a strict action of $C_{8}$ on $\left(A_{*}, F_{f}^{h}\right)$. With an eye toward Proposition 11.8, we invert the class $u$ and redefine $A_{*}$ to be $A\left[u, u^{-1}\right]$. The underlying $C_{2}$-action is the conjugation action, so Proposition 11.28 provides a $C_{8}$-equivariant map

$$
\pi_{*}^{u} M U^{\left(\left(C_{8}\right)\right)} \rightarrow A_{*},
$$

classifying $\left(A_{*}, F_{f}^{h}\right)$ with its $C_{8}$-action.

Proposition 11.31. The image of $D$ under

$$
D \in \pi_{19 \rho} M U^{\left(\left(C_{8}\right)\right)} \rightarrow \pi_{152}^{u} M U^{\left(\left(C_{8}\right)\right)} \rightarrow A_{152}
$$

is a unit, hence (11.30) factors through a $C_{8}$-equivariant map

$$
\pi_{*}^{u} \Omega_{\mathbb{O}} \rightarrow A_{*} .
$$

Proof: We must show that the classes $r_{1}^{C_{8}}, r_{3}^{C_{4}}$, and $r_{15}^{C_{2}}$ all map to units in $A_{*}$. It suffices to show that they do so in $A_{*} /(\pi)$. By definition (§5.4.2) the image of $r_{1}^{C_{8}}$ in $A_{2} /(\pi)$ is the coefficient of $x^{2}$ in the isomorphism of $\gamma^{*} F_{f}^{h}$ with the 2-typification of $F_{f}^{h}$. Since $F_{f}^{h}$ is already 2-typical, this is just the coefficient of $x^{2}$ in the power series $[\zeta]_{F_{f}^{h}}(x)$ in the homogeneous formal group law. By (11.15) this coefficient is 
congruent to $u$ modulo $\pi$, hence a unit. Equation (11.15) similarly shows that $r_{3}^{C_{4}}$ maps to $u^{3}$, and $r_{15}^{C_{2}}$ to $u^{15}$ modulo $(\pi)$. This completes the proof.

11.5. Technical results. In this section we describe explicitly the maps

$$
\begin{gathered}
\operatorname{Ext}_{M U_{*} M U}^{1,2^{j+1}}\left(M U_{*}, M U_{*} /(2)\right) \rightarrow \operatorname{Ext}_{\mathcal{A}}^{2,2^{j+1}}(\mathbb{Z} / 2, \mathbb{Z} / 2) \\
\operatorname{Ext}_{M U_{*} M U}^{1,2^{j+1}}\left(M U_{*}, M U_{*} /(2)\right) \rightarrow H^{1}\left(C_{8} ; A_{2^{j+1}} /(\pi)\right)
\end{gathered}
$$

occurring in the statement of Proposition 11.13. The results are Propositions 11.36 and 11.38 below. Combined with Lemma 11.11 and Proposition 11.34 they directly imply Proposition 11.13.

11.5.1. Preliminaries. We remind the reader that everything has been localized at the prime $p=2$. The 2-typification $F_{\text {univ }}^{(2)}$ of the universal formal group law $F_{\text {univ }}$ is classified by a map $B P_{*} \rightarrow M U_{*}$. This map extends to an equivalence of Hopf algebroids

$$
\left(B P_{*}, B P_{*} B P\right) \stackrel{\approx}{\longrightarrow}\left(M U_{*}, M U_{*} M U\right),
$$

giving an isomorphism

$$
\operatorname{Ext}_{B P_{*} B P}^{s, t}\left(B P_{*}, B P_{*}\right) \stackrel{\approx}{\rightarrow} \operatorname{Ext}_{M U_{*} M U}^{s, t}\left(M U_{*}, M U_{*}\right) .
$$

Our proofs depend heavily on the computations in [63] and [78], which are stated for $B P$. Because of the above isomorphism they apply equally well to $M U$.

In order to describe explicit computations, we fix the identification

$$
B P_{*} B P=\mathbb{Z}_{(2)}\left[v_{1}, v_{2}, \ldots, t_{1}, t_{2}, \ldots\right]
$$

in which the $v_{i}$ are the Hazewinkel generators, and the elements $t_{i} \in B P_{*} B P$ are the coefficients of the universal isomorphism

$$
\begin{aligned}
\eta_{R}^{*} F_{\text {univ }}^{(2)} & \rightarrow F_{\text {univ }}^{(2)}=\eta_{L}^{*} F_{\text {univ }}^{(2)} \\
x & \mapsto \sum^{F_{\text {univ }}^{(2)}} t_{n} x^{2^{n}}
\end{aligned}
$$

We will not distinguish in notation between the $v_{i}$ and $t_{i}$ in $B P_{*} B P$, and their images in $M U_{*} M U$.

An important role in the proof of 11.3 is played by the element $t_{1}$. Since any coordinate $x$ is 2-typical modulo $x^{3}$, the class $t_{1}$ is also given by the coefficient of the universal isomorphism of $\eta_{R}^{*} F_{\text {univ }}$ with $\eta_{L}^{*} F_{\text {univ }}$

$$
x \mapsto x+t_{1} x^{2}+\cdots \text {. }
$$

With the standard conventions this is the inverse of the universal strict isomorphism over $\pi_{*} M U \wedge M U$, which goes from $\eta_{L}^{*} F_{\text {univ }}$ to $\eta_{R}^{*} F_{\text {univ }}$.

\subsubsection{The Adams-Novikov 2-line. Let}

$$
\begin{aligned}
& \delta_{2}: \operatorname{Ext}_{M U_{*} M U}^{1,2^{j+1}}\left(M U_{*}, M U_{*} /(2)\right) \rightarrow \operatorname{Ext}_{M U_{*} M U}^{2,2^{j+1}}\left(M U_{*}, M U_{*}\right) \quad \text { and } \\
& \delta_{1}: \operatorname{Ext}_{M U_{*} M U}^{0,2^{j+1}}\left(M U_{*}, M U_{*} /\left(2, v_{1}^{\infty}\right)\right) \rightarrow \operatorname{Ext}_{M U_{*}, 2_{U}^{j+1}}^{1,1}\left(M U_{*}, M U_{*} /(2)\right)
\end{aligned}
$$

be the connecting homomorphisms associated to the short exact sequences of $M U_{*} M U$ co-modules

$$
\begin{gathered}
0 \rightarrow M U_{*} \stackrel{2}{\rightarrow} M U_{*} \rightarrow M U_{*} /(2) \rightarrow 0 \\
0 \rightarrow M U_{*} / 2 \rightarrow v_{1}^{-1} M U_{*} \rightarrow M U_{*} /\left(2, v_{1}^{\infty}\right) \rightarrow 0 .
\end{gathered}
$$


Our description of the maps (11.32) relies on the following computation, whose proof is discussed in $\S 11.6$. We employ the standard "cobar construction" notation for elements (see, for example, [71, Definition A1.2.11]).

Proposition $11.34([63,78])$. For $j>1$, the $\mathbb{Z} / 2$-vector space $\operatorname{Ext}_{M U_{*} M U}^{1,2^{j+1}}\left(M U_{*}, M U_{*} /(2)\right)$ has a basis consisting of the elements,

$$
v_{1}^{2^{j}-1}\left[t_{1}\right], \quad v_{1}^{2^{j}-2}\left[t_{1}^{2}\right]
$$

and the image under $\delta_{1}$ of certain elements of the form

$$
v_{2}^{s 2^{k}} / v_{1}^{2^{k}} \in \operatorname{Ext}_{M U_{*} M U}^{0,2^{j+1}}\left(M U_{*}, M U_{*} /\left(2, v_{1}^{\infty}\right)\right),
$$

with $s$ odd.

We will also need

Lemma 11.35. For $k \geq 2$ the connecting homomorphisms $\delta_{1}$ and $\delta_{2}$ satisfy the following congruences modulo the ideal $\left(2, v_{1}^{2}\right)$ :

$$
\begin{aligned}
\delta_{1}\left(v_{2}^{s 2^{k}} / v_{1}^{2^{k}}\right) & \equiv v_{2}^{(s-1) 2^{k}}\left[t_{1}^{2^{k+1}}\right] \\
\delta_{2} v_{1}^{2^{k}}\left[t_{1}\right] & \equiv 0 \\
\delta_{2} v_{1}^{2^{k}}\left[t_{1}^{2}\right] & \equiv 0 \\
\delta_{2} \delta_{1}\left(v_{2}^{s 2^{k}} / v_{1}^{2^{k}}\right) & \equiv v_{2}^{(s-1) 2^{k}}\left[t_{1}^{2^{k}} \mid t_{1}^{2^{k}}\right] .
\end{aligned}
$$

Proof: This is a straightforward (and long-known) computation using the structure formulae

$$
\begin{aligned}
& \eta_{R}\left(v_{1}\right)=v_{1}+2 t_{1} \\
& \eta_{R}\left(v_{2}\right) \equiv v_{2}+v_{1} t_{1}^{2}+v_{1}^{2} t_{1} \quad \bmod 2 .
\end{aligned}
$$

The assertion about $\delta_{1}$ is easy to check. The structure formulae imply that $\eta_{R}\left(v_{1}^{2}\right) \equiv$ $v_{1}^{2}$ modulo 4 , so one may work modulo $\left(4, v_{1}^{2}\right)$ when computing $\delta_{2}$. The terms $v_{1}^{2^{k}}\left[t_{1}\right]$ and $v_{1}^{2^{k}}\left[t_{1}^{2}\right]$ are already in this ideal, giving the first two assertions about $\delta_{2}$. The last makes use of the congruences

$$
\begin{aligned}
\delta_{2}\left[t_{1}^{2^{k+1}}\right] & \equiv\left[t_{1}^{2^{k}} \mid t_{1}^{2^{k}}\right] \quad \bmod 2 \\
\eta_{R} v_{2}^{4 i} & \equiv v_{2}^{4 i} \quad \bmod \left(4, v_{1}^{2}\right) .
\end{aligned}
$$

Since $s$ is odd and $k \geq 1,(s-1) 2^{k}$ is divisible by 4 . This means that

$$
\delta_{2}\left(v_{2}^{(s-1) 2^{k}}\left[t_{1}^{2^{k+1}}\right]\right) \equiv v_{2}^{(s-1) 2^{k}} \delta_{2}\left(\left[t_{1}^{2^{k+1}}\right]\right) \equiv v_{2}^{(s-1) 2^{k}}\left[t_{1}^{2^{k}} \mid t_{1}^{2^{k}}\right] \bmod \left(2, v_{1}^{2}\right) .
$$

This completes the proof.

11.5.3. The proof of Proposition 11.13. Given Lemma 11.11 and Proposition 11.34, Proposition 11.13 is an immediate consequence of the two results below.

Proposition 11.36. For $j>1$, the map

$$
\operatorname{Ext}_{M U_{*} M U}^{1,2^{j+1}}\left(M U_{*}, M U_{*}\right) \rightarrow \operatorname{Ext}_{\mathcal{A}}^{2,2^{j+1}}(\mathbb{Z} / 2, \mathbb{Z} / 2) .
$$


is given by

$$
\begin{aligned}
v_{1}^{2^{j}-1}\left[t_{1}\right] & \mapsto 0 \\
v_{1}^{2^{j}-2}\left[t_{1}^{2}\right] & \mapsto 0 \\
\delta_{1}\left(v_{2}^{s 2^{k}} / v_{1}^{2^{k}}\right) & \mapsto 0 \quad(s>1) \\
\delta_{1}\left(v_{2}^{2^{j-1}} / v_{1}^{2^{j-1}}\right) & \mapsto h_{j}^{2} .
\end{aligned}
$$

Proof: This follows directly from Lemma 11.35 and the fact that the map from $M U_{*} M U$ to the dual Steenrod algebra given by

$$
\begin{aligned}
v_{i} & \mapsto 0 \\
t_{i} & \mapsto \chi\left(\xi_{i}\right)^{2} .
\end{aligned}
$$

Remark 11.37. The map from the Adams-Novikov $E_{2}$-term to the classical Adams $E_{2}$-term has been completely determined for $s \leq 2$ and all $t$. For a comprehensive discussion, the reader is referred to [71, Chapter 5].

We next turn to the second map in Proposition 11.13. When $j>2$ the action of $C_{8}$ on $A_{2^{j+1}}$ is trivial, and the group $H^{1}\left(C_{8} ; A_{2^{j+1}} /(\pi)\right)$ is cyclic of order two, generated by the cohomology class of the cocycle whose value on $\gamma$ is $u^{2^{j}}$. Let us denote this class $v$.

Proposition 11.38. For $j>2$, the map

$$
\operatorname{Ext}_{M U_{*} M U}^{1,2^{j+1}}\left(M U_{*}, M U_{*} /(2)\right) \rightarrow H^{1}\left(C_{8} ; A_{2^{j+1}} /(\pi)\right) .
$$

is given by

$$
\begin{aligned}
& v_{1}^{2^{\jmath}-1}\left[t_{1}\right] \mapsto 0 \\
& v_{1}^{2^{j}-2}\left[t_{1}^{2}\right] \mapsto 0 \\
& \delta_{1}\left(v_{2}^{s 2^{k}} / v_{1}^{2^{k}}\right) \mapsto 0 \quad(s>1) \\
& \delta_{1}\left(v_{2}^{2^{j-1}} / v_{1}^{2^{j-1}}\right) \mapsto v \text {. }
\end{aligned}
$$

Proof: Let $\nu$ be the valuation on $A$ normalized so that $\nu(\pi)=1$. Since $\nu(2)=4$,

$$
[2]_{F_{f}^{h}}(x) \equiv x^{2^{4}}+\cdots \quad \bmod \pi,
$$

and $v_{1}$ and $v_{2}$ both map to zero in $A_{*} /(\pi)$. This gives the first line, and makes the second a consequence of Lemma 11.35. Lemma 11.35 also gives the identity

$$
\delta_{1}\left(v_{2}^{2^{j}} / v_{1}^{2^{j}}\right)=\left[t_{1}^{2^{j}}\right]
$$

so to determine the image of $\delta_{1}\left(v_{2}^{2^{j}} / v_{1}^{2^{j}}\right)$ we need to work out the image of $t_{1}$ under the map of Hopf-algebroids

$$
\left(M U_{*}, M U_{*} M U\right) \rightarrow\left(A_{*} /(\pi), C\left(C_{8}, A_{*} /(\pi)\right)\right) .
$$

As explained at the end of 11.5.1, the element $t_{1}$ occurs as the coefficient of $x^{2}$ the isomorphism $\eta_{R}^{*} F_{\text {univ }}^{(2)} \rightarrow F_{\text {univ }}^{(2)}$, inverse to the universal strict isomorphism. Since we have chosen a 2 -typical coordinate on $F_{f}^{h}$, the element $t_{1}$ is therefore sent, under 
the map of Hopf-algebroids (11.39), to the 1-cocycle on $C_{8}$ whose value on $\gamma$ is the coefficient of $x^{2}$ in the inverse of the power series $[\zeta](x)$. By (11.15) and the formula for strictification (11.17) this is $-u^{2^{j}} \equiv u^{2^{j}}$ modulo $(\pi)$.

11.6. Addendum. Lemma 11.11 and Proposition 11.34 do not quite appear literature in a readily accessible form, and the purpose of this addendum is to outline their proofs, explaining how the key points can be read off from the results of [63] and [78]. To conform with the notation of these references, we will use $B P$ rather that $M U$ in this section.

The paper [63] introduced the chromatic approach to studying the groups

$$
\operatorname{Ext}_{B P_{*} B P}^{s, *}\left(B P_{*}, B P_{*}\right) .
$$

The computation begins with the fact that for $s>0$ one has

$$
\operatorname{Ext}_{B P_{*} B P}^{s, *}\left(B P_{*}, B P_{*}\right) \otimes \mathbb{Q}=0 .
$$

This means that the connecting homomorphism

$$
\operatorname{Ext}_{B P_{*} B P}^{1,2^{j+1}}\left(B P_{*}, B P_{*} / 2^{\infty}\right) \rightarrow \operatorname{Ext}_{B P_{*} B P}^{2,2^{j+1}}\left(B P_{*}, B P_{*}\right)
$$

is an isomorphism. The assertion of Proposition 11.34 is that the map

$$
\operatorname{Ext}_{B P_{*} B P}^{1,2^{j+1}}\left(B P_{*}, B P_{*} / 2\right) \rightarrow \operatorname{Ext}_{B P_{*} B P}^{1,2^{j+1}}\left(B P_{*}, B P_{*} / 2^{\infty}\right)
$$

induced by the inclusion

$$
B P_{*} / 2 \stackrel{1 / 2}{\longrightarrow} B P_{*} / 2^{\infty}
$$

is surjective, and that the left had group is spanned by the elements listed. Continuing with the chromatic approach, one is led to the following diagram (in which, to manage the size, we have abbreviated $\operatorname{Ext}_{B P_{*} B P}^{s, t}\left(B P_{*}, M\right)$ to $\left.\operatorname{Ext}^{s, t}(M)\right)$

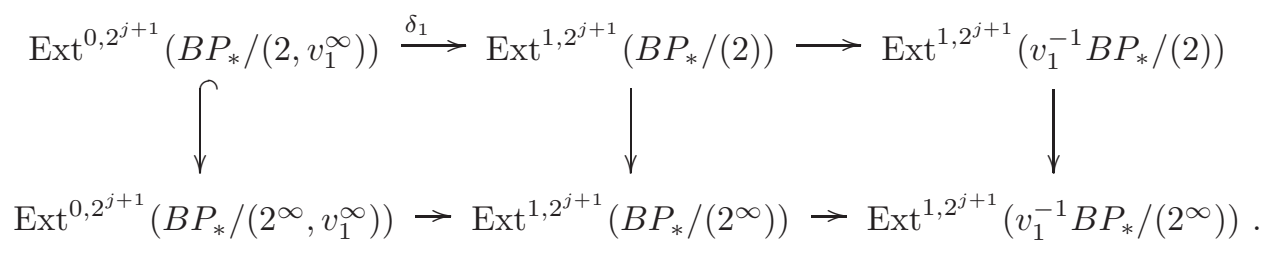

The rightmost column is analyzed using the Miller-Ravenel change of rings theorem (see [62] or [71, Chapter 6, §1]) which identifies it with the map

$$
H^{1}\left(\mathbb{Z}_{2}^{\times} ; \mathbb{Z}_{(2)}\left[v_{1}^{ \pm 1}\right] /(2)\right) \rightarrow H^{1}\left(\mathbb{Z}_{2}^{\times} ; \mathbb{Z}_{(2)}\left[v_{1}^{ \pm 1}\right] /\left(2^{\infty}\right)\right)
$$

in which $\lambda \in \mathbb{Z}_{2}^{\times}$acts on $v_{1}$ with eigenvalue $\lambda$. This is easily calculated, and one finds that the map is indeed surjective, and that $\operatorname{Ext}_{B P_{*} B P}^{1,2^{j+1}}\left(B P_{*}, v_{1}^{-1} B P_{*} /(2)\right)$ has dimension 2, with basis the image of $v_{1}^{2^{j-1}}\left[t_{1}\right]$ and $v_{1}^{2^{j-2}}\left[t_{1}^{2}\right]$. This reduces Proposition 11.34 to the assertion that the left vertical arrow is surjective (hence an isomorphism), and that the upper left group has a basis consisting of the elements of the form $v_{2}^{s 2^{k}} / v_{1}^{2^{k}}$. For this one first appeals to the invariant prime ideal theorem $([66,44]$, or see $[71$, Theorem 4.3.2]) for the fact that

$$
\operatorname{Ext}_{B P_{*} B P}^{0, *}\left(B P_{*}, B P_{*} /\left(2, v_{1}\right)\right)=\mathbb{Z} / 2\left[v_{2}\right] .
$$


It follows that any invariant element in $B P_{*} B P /\left(2^{\infty}, v_{1}^{\infty}\right)$ has the form

$$
\frac{v_{2}^{s 2^{k}}+r}{v_{1}^{\ell} 2^{i+1}}
$$

with $r \in\left(2, v_{1}\right) \subset B P_{*}$. We now come to the key point. It turns out that a necessary (but not sufficient) condition that such an element to be invariant is that the indices satisfy the inequality

$$
\ell \leq 2^{k-i}+2^{k-i-1} .
$$

This can be extracted from the stronger conditions of [78, Theorem 3.3], in which the symbol $x_{n}$ is an explicitly defined element, congruent to $v_{2}^{2^{n}}$ modulo $\left(2, v_{1}\right)$ and $y_{i}$ is an explicitly defined element congruent to $v_{1}^{2^{i}}$ modulo 2. From (11.42) it follows that for an element of the form (11.41) to be invariant and have degree $2^{j+1}$, the numbers $i, j, k$, and $\ell$ must satisfy

$$
6 s 2^{k}-2\left(2^{k-i}+2^{k-i-1}\right) \leq 2^{j+1} \leq 6 s 2^{k} .
$$

Expanding, and dividing both sides by $2^{k+1}$ gives

$$
3 s-2^{-i}-2^{-i-1} \leq 2^{j-k} \leq 3 s .
$$

Since $s \geq 1$ and $i \geq 0$, one has

$$
3 s-2^{-i}-2^{-i-1} \geq 3 s-3 / 2>1,
$$

and so $k<j$. This implies $2^{j-k}$ is even, and so must equal $3 s-1$. This in turn means that $2^{-i}+2^{-i-1}>1$, and so $i$ must be 0 .

It thus follows from the inequality (11.42) that the invariant elements of degree $2^{j+1}$ in $B P_{*} /\left(2^{\infty}, v_{1}^{\infty}\right)$ have the form

$$
\frac{v_{2}^{s 2^{k}}+r}{v_{1}^{2^{k}} 2} .
$$

Thus the left vertical map in (11.40) is surjective. Since

$$
\frac{v_{2}^{s 2^{k}}}{v_{1}^{2^{k}} 2}
$$

is already invariant, a simple induction shows that the elements stated form a basis.

\section{Appendix A. The CATEgory of EQUivariant orthogonal SPECTRA}

In this appendix we recall the definition and some basic properties of the theory of equivariant orthogonal spectra. For further details and references the reader is referred to Mandell-May [53] and to Mandell-May-Schwede-Shipley [54].

One of the reasons we have chosen to use equivariant orthogonal spectra is that it has many convenient category theoretic properties. These are independent of the homotopy theory of equivariant orthogonal spectra, and so we make two passes through the theory, one focused on the category theory, and the other on the homotopy theory.

Our main new innovation is the theory of the norm (§A.4). Most of the category theoretic aspects apply to any symmetric monoidal category, and things work out much cleaner at that level of generality.

\section{A.1. Category theory preliminaries.}




\section{A.1.1. Symmetric monoidal categories.}

Definition A.1. A symmetric monoidal category is a category $\mathcal{V}$ equipped with a functor

$$
\otimes: \mathcal{V} \times \mathcal{V} \rightarrow \mathcal{V}
$$

a unit object $\mathbf{1} \in \mathcal{V}$, a natural associativity isomorphism

$$
a_{A B C}:(A \otimes B) \otimes C \approx A \otimes(B \otimes C)
$$

a natural commutativity isomorphism

$$
s_{A B}: A \otimes B \approx B \otimes A
$$

and a unit isomorphism

$$
\iota_{A}: \mathbf{1} \otimes A \approx A .
$$

This data is required to satisfy the associative and commutative coherence axioms, as well as the strict symmetry axiom.

The two coherence axioms express that all of the ways one might get from one iterated tensor product to another using the associativity and commutativity transformations coincide. The strict symmetry axiom is that the square of the commutativity transformation is the identity map. See [51], or Borceux [12, §6.1].

Even though it requires six pieces of data to specify a symmetric monoidal category we will usually indicate one with a triple $\mathcal{V}=\left(\mathcal{V}_{0}, \otimes, \mathbf{1}\right)$.

A symmetric monoidal category is closed if for each $A$, the functor $A \otimes(-)$ has a right adjoint $(-)^{A}$, which one can think of as an "internal hom." Note that

$$
\mathcal{V}\left(\mathbf{1}, X^{A}\right) \approx \mathcal{V}(A, X)
$$

so that one can recover the usual hom from the internal hom.

A.1.2. Sifted colimits, commutative and associative algebras. In a closed symmetric monoidal category, the monoidal product commutes with colimits in each variable. It follows easily that the iterated monoidal product

$$
X \mapsto X^{\otimes n}
$$

commutes with all colimits over indexing categories $I$ for which the diagonal $I \rightarrow I^{n}$ is final in the sense of [51, §IX.3]. If $I \rightarrow I \times I$ is final, then for all $n \geq 2, I \rightarrow I^{n}$ is also final.

Definition A.2. A category $I$ is sifted the diagonal embedding $I \rightarrow I \times I$ is final.

Equivalently (see [25, 15.2 (c)], or [2, Theorem 2.15]), a small category $I$ is sifted if and only if the formation of colimits over $I$ in sets commutes with finite products.

Definition A.3. A sifted colimit is a colimit over a sifted category.

Examples of sifted colimits include reflexive coequalizers and directed colimits. In fact the class of sifted colimits is essentially the smallest class of colimits containing reflexive coequalizers and directed colimits. See, for example [1, 25].

Let $\mathcal{V}=\left(\mathcal{V}_{0}, \otimes, \mathbf{1}\right)$ be a closed symmetric monoidal category.

Definition A.4. An associative algebra in $\mathcal{V}$ is an object $A$ equipped with a multiplication map $A \otimes A \rightarrow A$ which is unital and associative. A commutative algebra is an associative algebra for which the multiplication map is commutative. 
The categories of associative and commutative algebras (and algebra maps) in $\mathcal{V}$ are denoted ass $\mathcal{V}$ and $\operatorname{comm} \mathcal{V}$, respectively.

The following straightforward result holds more generally for algebras over any operad. The existence of colimits in the algebra categories is proved by expressing any algebra as a reflexive coequalizer of a diagram of free algebras. There is an even more general result for algebras over a triple [12, Proposition 4.3.1]

Proposition A.5. Suppose that $\mathcal{V}$ is a closed symmetric monoidal category. The forgetful functors

$$
\begin{aligned}
\operatorname{ass} \mathcal{V} & \rightarrow \mathcal{V} \\
\operatorname{comm} \mathcal{V} & \rightarrow \mathcal{V}
\end{aligned}
$$

create limits. If $\mathcal{V}$ is cocomplete these functors have left adjoints

$$
\begin{aligned}
& X \mapsto T(X)=\coprod_{n \geq 0} X^{\otimes n} \\
& X \mapsto \operatorname{Sym}(X)=\coprod_{n \geq 0} X^{\otimes n} / \Sigma_{n},
\end{aligned}
$$

the categories ass $\mathcal{V}$ and $\operatorname{comm} \mathcal{V}$ are cocomplete, and the "free" functors above commute with all sifted colimits.

A left module over an associative algebra $A$ is an object $M$ equipped with a unital and associative left multiplication

$$
A \otimes M \rightarrow M .
$$

Similarly a right module is an object $N$ equipped with a unital, associative right multiplication $N \otimes A \rightarrow N$. Given a left $A$-module $M$ and a right $A$-module $N$ one defines $N \underset{A}{\otimes} M$ by the (reflexive) coequalizer

$$
N \otimes A \otimes M \rightrightarrows N \otimes M \rightarrow N \underset{A}{\otimes} M .
$$

When $A$ is commutative, a left $A$-module can be regarded as a right $A$-module by the action

$$
M \otimes A \stackrel{\text { flip }}{\longrightarrow} A \otimes M \rightarrow M .
$$

Using this, the formation $M \underset{A}{\otimes} N$ makes the category of left $A$-modules into a symmetric monoidal category.

A.1.3. Enriched categories. In this section we briefly describe the basic notions of enriched categories.. The reader is referred to [40] or [12, Ch. 6] for further details.

Suppose that $\mathcal{V}=\left(\mathcal{V}_{0}, \otimes, \mathbf{1}\right)$ is a symmetric monoidal category.

Definition A.6. A $\mathcal{V}$-category $\mathcal{C}$ consists of a collection ob $\mathcal{C}$ called the objects of $\mathcal{C}$, for each pair $X, Y \in$ ob $\mathcal{C}$ a morphism object $\mathcal{C}(X, Y) \in$ ob $\mathcal{V}_{0}$, for each $X$ an identity morphism $\mathbf{1} \rightarrow \mathcal{C}(X, X)$ and for each triple $X, Y, Z$ of objects of $\mathcal{C}$ a composition law

$$
\mathcal{C}(Y, Z) \otimes \mathcal{C}(X, Y) \rightarrow \mathcal{C}(X, Z)
$$

This data is required to satisfy the evident unit and associativity properties. 
As is customary, we write $X \in \mathcal{C}$ rather than $X \in \mathrm{ob} \mathcal{C}$. Most of the notions of ordinary category theory carry through in the context of enriched categories, once formulated without reference to "elements" of mapping objects. For example a functor $F: \mathcal{C} \rightarrow \mathcal{D}$ of $\mathcal{V}$-categories consists of a function

$$
F: \mathrm{ob} \mathcal{C} \rightarrow \mathrm{ob} \mathcal{D}
$$

and for each pair of objects $X, Y \in \mathcal{C}$ a $\mathcal{V}$-morphism

$$
F: \mathcal{C}(X, Y) \rightarrow \mathcal{D}(F X, F Y)
$$

compatible with the unit and composition. A natural transformation between two functors $F$ and $G$ is a function assigning to each $X \in \mathcal{C}$ a map $T_{X}: \mathbf{1} \rightarrow \mathcal{D}(F X, G X)$ which for every $X, Y$ makes the diagram

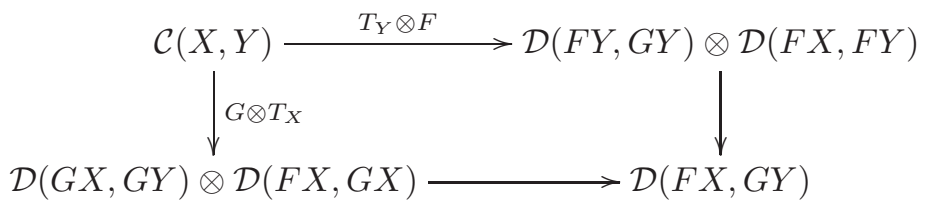

commute.

There is an ordinary category $\mathcal{C}_{0}$ underlying the enriched category $\mathcal{C}$. The objects of $\mathcal{C}_{0}$ are the objects of $\mathcal{C}$, and one defines

$$
\mathcal{C}_{0}(X, Y)=\mathcal{V}_{0}(\mathbf{1}, \mathcal{C}(X, Y))
$$

If $\mathcal{V}$ itself underlies a $\mathcal{W}$-enriched category, then any $\mathcal{V}$-category $\mathcal{C}$ has an underlying $\mathcal{W}$-category, whose underlying ordinary category is $\mathcal{C}_{0}$.

When $\mathcal{V}$ is a closed symmetric monoidal category, the internal hom defines an enrichment of $\mathcal{V}$ over itself, with underlying category $\mathcal{V}_{0}$.

When $\mathcal{V}$ is closed, a natural transformation $F \rightarrow G$ can be described as a map

$$
1 \rightarrow \prod_{X \in \mathcal{C}} \mathcal{D}(F X, G X)
$$

which equalizes the two arrows

$$
\prod_{X \in \mathcal{C}} \mathcal{D}(F X, G X) \rightrightarrows \prod_{X, Y \in \mathcal{C}} \mathcal{D}(F X, G Y)^{\mathcal{C}(X, Y)}
$$

describing the two ways of going around (A.7).

We will write $\mathfrak{C a t}_{\mathcal{V}}$ for the 2-category of $\mathcal{V}$-categories, and denote the category of enriched functors $\mathcal{C} \rightarrow \mathcal{D}$ as $\mathfrak{C a t}_{\mathcal{V}}(\mathcal{C}, \mathcal{D})_{0}$. When $\mathcal{V}$ is closed and contains products indexed by the collection of pairs of objects of $\mathcal{C}$, the category $\mathfrak{C a t} \mathcal{V}(\mathcal{C}, \mathcal{D})_{0}$ underlies an enriched category $\mathfrak{C a t}_{\mathcal{V}}(\mathcal{C}, \mathcal{D})$ in which the object of natural transformations from $F$ to $G$ is given by the equalizer of (A.8).

\section{A.2. Equivariant orthogonal spectra.}

A.2.1. Equivariant spaces. Let $\mathcal{T}$ be the category of pointed, compactly generated weak Hausdorff spaces (in the sense of [59]). The category $\mathcal{T}$ is symmetric monoidal under the smash product, with unit $S^{0}$. A topological category is a category enriched over $\left(\mathcal{T}, \wedge, S^{0}\right)$. 
Remark A.9. Working with compactly generated weak Hausdorff spaces has many benefits, but it does create some technical issue. Colimits are computed by forming the colimit in topological spaces, replacing the topology by the compactly generated topology, and then forming the universal quotient which is weak Hausdorff. This last step can alter the underlying point set. It does not, however, in the case of pushouts along closed inclusion. More precisely, given a pushout diagram

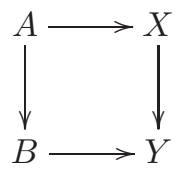

of topological spaces in which $A \rightarrow X$ is a closed inclusion, if $A, X$, and $B$ are compactly generated and weak Hausdorff then so is $Y$. This follows from [59, Proposition 2.5] and the remark about adjunction spaces immediately preceding its statement. Among other things this means that the smash product of two compactly generated weak Hausdorff spaces can be computed as the smash product of the underlying compactly generated spaces.

Now suppose that $G$ is a group. Let $\left(\mathcal{T}^{G}, \wedge, S^{0}\right)$ be the topological symmetric monoidal category of pointed spaces with a left $G$-action and spaces of equivariant maps. With this structure $\mathcal{T}^{G}$ is a closed symmetric monoidal category, with internal mapping spaces $\mathcal{I}_{G}(X, Y)=Y^{X}$ given by the space of non-equivariant maps, with the conjugation action of $G$.

A word about notation. The expression "category of $G$-spaces" can reasonably refer to three objects, depending on what is meant by a map. It can be an ordinary category, a category enriched over topological spaces, or a category in which the hom objects are the $G$-spaces of non-equivariant maps. As indicated above we will use $\mathcal{I}_{G}$ to denote the category enriched over $G$-spaces, with $\mathcal{I}_{G}(X, Y)$ denoting the $G$-space of non-equivariant maps, and $\mathcal{T}^{G}$ for the topological category of $G$-spaces, and spaces of equivariant maps.

We will be making use of categories enriched over $\mathcal{T}^{G}$. As in [53], we will refer to them as topological $G$-categories (or just $G$-categories for short). Let $\mathfrak{C a t}_{G}$ denote the collection of topological $G$-categories, and write $\mathfrak{C a t}_{G}(\mathcal{C}, \mathcal{D})$ for the enriched category of functors and left $G$-spaces of natural transformations. The symbol $\mathfrak{C a t}_{G}(\mathcal{C}, \mathcal{D})^{G}$ will denote the topological category of functors and spaces of equivariant natural transformations.

A.2.2. Change of group. Suppose that $H \subset G$ is a subgroup. The restriction functor

$$
\mathcal{T}^{G} \rightarrow \mathcal{T}^{H}
$$

has continuous left and a right adjoints given by

$$
\begin{aligned}
Y \mapsto G_{+}{ }_{H}^{\wedge} Y \\
Y \mapsto \mathcal{T}^{H}\left(G_{+}, Y\right) .
\end{aligned}
$$

These two constructions are basic examples of indexed monoidal products (see $\S A .3)$. Because $\mathcal{T}^{G}$ is pointed there is a canonical equivariant map

$$
G_{+}{ }_{H} Y \rightarrow \mathcal{T}^{H}\left(G_{+}, Y\right) .
$$


A.2.3. The basic indexing categories. For a real orthogonal representation $V$ of $G$ let $O(V)$ be the orthogonal group of non-equivariant linear isometric maps $V \rightarrow V$. The group $G$ acts on $O(V)$ by conjugation, and the group of fixed points is the orthogonal group of equivariant maps. Given orthogonal representations $V$ and $W$, we define $O(V, W)$ to be the Stiefel manifold of linear isometric embeddings of $V$ into $W$, with the conjugation action of $G$. The $G$ fixed points in $O(V, W)$ are the equivariant orthogonal embeddings. The group $O(W)$ acts transitively on $O(V, W)$ on the left. A choice of embedding $V \rightarrow W$ identifies $O(V, W)$ with the homogeneous space $O(W) / O(W-V)$.

Definition A.10. The category $\mathscr{J}_{G}$ is the topological $G$-category whose objects are finite dimensional real orthogonal representations of $G$, and with morphism $G$-space $\mathscr{J}_{G}(V, W)$ the Thom complex

$$
\mathscr{J}_{G}(V, W)=\operatorname{Thom}(O(V, W) ; W-V)
$$

of the "complementary bundle" $W-V$ over $O(V, W)$.

We will denote the topological category underlying $\mathscr{J}_{G}$ with the symbol $\mathscr{J}^{G}$. Thus $\mathscr{J}^{G}(V, W)=\mathscr{J}_{G}(V, W)^{G}$.

The $G$-space $\mathscr{J}_{G}(V, W)$ can be thought of as the topologically indexed wedge

$$
\bigvee_{V \rightarrow W} S^{W-V}
$$

When $\operatorname{dim} W<\operatorname{dim} V$ it reduces to the one point space $*$. When $\operatorname{dim} W \geq \operatorname{dim} V$ one can get a convenient description by choosing an orthogonal $G$-representation $U$ with $\operatorname{dim} U+\operatorname{dim} V=\operatorname{dim} W$ (for example the trivial representation). With this choice one has

$$
\mathscr{J}_{G}(V, W) \approx O(V \oplus U, W)_{+} \underset{O(U)}{\wedge} S^{U} .
$$

The fixed point space $\mathscr{J}_{G}(V, W)^{G}$ is given by

$$
\mathscr{J}_{G}(V, W)^{G}=\mathscr{J}\left(V^{G}, W^{G}\right) \wedge O\left(V^{\perp}, W^{\perp}\right)_{+}^{G},
$$

in which $V^{G}$ denotes space of invariant vectors in $V$, and $V^{\perp}$ its orthogonal complement. The space $O\left(V^{\perp}, W^{\perp}\right)^{G}$ in turn decomposes into the product

$$
\prod_{\alpha} O\left(V_{\alpha}, W_{\alpha}\right)
$$

in which $\alpha$ is running through the set of non-trivial irreducible representations of $G$, and $V_{\alpha} \subset V$ and $W_{\alpha} \subset W$ indicate the $\alpha$-isotypical parts.

When $G$ is the trivial group we will denote the category $\mathscr{J}_{G}$ simply by $\mathscr{J}$. For any $G$ there is an inclusion $\mathscr{J} \subset \mathscr{J}_{G}$ identifying $\mathscr{J}$ with the full subcategory of objects with trivial $G$-action. There is also a forgetful functor $\mathscr{J}_{G} \rightarrow \mathscr{J}$ which refines in the evident manner to a functor from $\mathscr{J}_{G}$ to the $G$-category of objects in $\mathscr{J}$ equipped with a $G$-action. One can easily check that this is an equivalence. For later reference, we single this statement out.

Proposition A.12. The forgetful functor described above gives an equivalence of $\mathscr{J}_{G}$ with the topological $G$-category of objects in $\mathscr{J}$ equipped with a $G$-action. Passage to fixed points gives an equivalence of $\mathscr{J}^{G}$ with the topological category of objects in $\mathscr{J}$ equipped with a $G$-action.

Proposition A.12 plays an important role in establishing one of the basic properties of the norm (Proposition A.59). 
A.2.4. Orthogonal spectra.

Definition A.13. An orthogonal G-spectrum is a functor

$$
\mathscr{J}_{G} \rightarrow \underline{\mathcal{I}}_{G}
$$

of topological $G$-categories.

Informally, an orthogonal spectrum $X$ consists of a collection of spaces $\left\{X_{V}\right\}$, and for each $V \rightarrow W$ a

$$
S^{W-V} \wedge X_{V} \rightarrow X_{W}
$$

These maps are required to be compatible with composition in $\mathscr{J}_{G}$, the action of $G$, and to vary continuously in $V \rightarrow W$. More formally, one has equivariant maps

$$
\operatorname{Thom}\left(O(V, W) ; S^{W-V}\right) \wedge X_{V} \rightarrow X_{W}
$$

compatible with composition.

Definition A.14. The topological G-category of orthogonal G-spectra is the category

The (topological) category of $G$-spectra is

$$
\underline{\mathfrak{S}}_{G}=\mathfrak{C a t}_{G}\left(\mathscr{J}_{G}, \mathcal{I}_{G}\right)
$$

$$
\mathcal{S}^{G}=\mathfrak{C a t}_{G}\left(\mathscr{J}_{G}, \underline{\mathcal{I}}_{G}\right)^{G} .
$$

We will use the notation

$$
\mathcal{S}=\mathfrak{C a t}_{G}(\mathscr{J}, \mathcal{T})
$$

to denote the category $\underline{S}_{G}$ for the case of the trivial group.

The $(G$-) category of orthogonal $G$-spectra is complete and cocomplete (in the sense of enriched categories). Both limits and colimits in $\mathcal{S}^{G}$ are computed objectwise:

$$
\begin{aligned}
& \left(\lim _{\longrightarrow} X^{\alpha}\right)_{V}=\lim _{\longrightarrow} X_{V}^{\alpha} \\
& \left(\lim _{\longleftarrow} X^{\alpha}\right)_{V}=\lim _{\longleftarrow} X_{V}^{\alpha} .
\end{aligned}
$$

Certain orthogonal $G$-spectra play a fundamental role. For $V \in \mathscr{J}_{G}$ let

$$
S^{-V}: \mathscr{J}_{G} \rightarrow \underline{\mathcal{I}}_{G}
$$

be the functor co-represented by $V$. By the Yoneda lemma

$$
\underline{\mathcal{S}}_{G}\left(S^{-V}, X\right)=X_{V} \text {. }
$$

For a pointed $G$-space $A$ let $S^{-V} \wedge A$ be the orthogonal $G$-spectrum with

$$
\left(S^{-V} \wedge A\right)_{W}=\left(S^{-V}\right)_{W} \wedge A \text {. }
$$

Again, by Yoneda,

$$
\underline{\mathcal{S}}_{G}\left(S^{-V} \wedge A, X\right)=\underline{\mathcal{T}}_{G}\left(A, X_{V}\right) .
$$

It also follows from the Yoneda lemma that every $X$ is functorially expressed as a reflexive coequalizer

$$
\bigvee_{V, W} S^{-W} \wedge \mathscr{J}_{G}(V, W) \wedge X_{V} \rightrightarrows \bigvee_{V} S^{-V} \wedge X_{V} \rightarrow X
$$

We call this the tautological presentation of $X$ and for ease of typesetting, sometimes indicate it as

$$
X=\underset{V}{\lim } S^{-V} \wedge X_{V}
$$


A.2.5. Smash product. The symmetric monoidal structures on $\mathscr{J}_{G}$ and $\mathcal{T}_{G}$ combine to give $\mathcal{S}^{G}$ a symmetric monoidal structure (the Day convolution), denoted $\wedge$. The smash product of two orthogonal $G$-spectra $X$ and $Y$ is defined to be the left Kan extension of

$$
(V, W) \mapsto X_{V} \wedge Y_{W}: \mathscr{J}_{G} \times \mathscr{J}_{G} \rightarrow \underline{\mathcal{T}}_{G}
$$

along the map

$$
\mathscr{J}_{G} \times \mathscr{J}_{G} \rightarrow \mathscr{J}_{G}
$$

sending $(V, W)$ to $V \oplus W$. The smash product is thus characterized by the fact that it commutes with enriched colimits in both variables, and satisfies

$$
S^{-V} \wedge S^{-W}=S^{-(V \oplus W)} .
$$

In terms of the tautological presentations

$$
\begin{aligned}
& X=\underset{V}{\lim _{W}} S^{-V} \wedge X_{V} \\
& Y=\underset{W}{\lim } S^{-W} \wedge Y_{W}
\end{aligned}
$$

one has

$$
\begin{aligned}
& X \wedge Y=\underset{V}{\lim } S^{-V} \wedge X_{V} \wedge Y \\
& =\underset{V}{\lim } S^{-V} \wedge X_{V} \wedge \underset{W}{\lim _{W}} S^{-W} \wedge Y_{W} \\
& =\underset{V, W}{\lim _{V, W}} S^{-V \oplus W} \wedge X_{V} \wedge Y_{W} .
\end{aligned}
$$

The above expression is, of course, an abbreviation for the reflexive coequalizer diagram

$$
\begin{aligned}
& \bigvee_{\substack{V_{0}, V_{1}, W_{0}, W_{1}}} \mathscr{J}_{G}\left(V_{0}, V_{1}\right) \wedge \mathscr{J}_{G}\left(W_{0}, W_{1}\right) \wedge S^{-V_{1} \oplus W_{1}} \wedge X_{V_{0}} \wedge Y_{W_{0}} \\
& \rightrightarrows \bigvee_{V, W} S^{-V \oplus W} \wedge X_{V} \wedge Y_{W} .
\end{aligned}
$$

Proposition A.17. The category $\mathcal{S}^{G}$ is a closed symmetric monoidal category with respect to $\wedge$.

Smashing the tautological presentation of a general spectrum $X$ with $S^{-V}$ gives a presentation of $S^{-V} \wedge X$ as a (reflexive) coequalizer

$$
\bigvee_{W_{0}, W_{1}} \mathscr{J}_{G}\left(W_{0}, W_{1}\right) \wedge S^{-V \oplus W_{1}} \wedge X_{W_{0}} \rightrightarrows \bigvee_{W} S^{-V \oplus W} \wedge X_{W} \rightarrow S^{-V} \wedge X
$$

This is not the tautological presentation of $S^{-V} \wedge X$, but from it, one can read off the formula of the following lemma

Lemma A.18. If $\operatorname{dim} W<\operatorname{dim} V$, then $\left(S^{-V} \wedge X\right)_{W}=*$. If $\operatorname{dim} W \geq \operatorname{dim} V$, then there is a natural isomorphism of $G$-spaces

$$
\left(S^{-V} \wedge X\right)_{W} \approx O(V \oplus U, W)_{+} \wedge_{O(U)} X_{U}
$$

where $U$ is any orthogonal G-representation with

$$
\operatorname{dim} U+\operatorname{dim} V=\operatorname{dim} W .
$$


A.2.6. Variations on the indexing category. There is a lot of flexibility in defining $\mathcal{S}^{G}$. In this section we describe a variation which is especially convenient for certain category theoretical properties, and will be used in our construction of the norm. We learned of the result below from Lars Hesselholt and Mark Hovey. It is due to Mandell-May ([53, Lemma V.1.5]).

Proposition A.19. Let $i: \mathscr{J} \rightarrow \mathscr{J}_{G}$ be the inclusion of the full subcategory of trivial $G$-representations. The functors

$$
i^{*}: \mathfrak{C a t}_{G}\left(\mathscr{J}_{G}, \underline{\mathcal{I}}_{G}\right) \rightarrow \mathfrak{C a t}_{G}\left(\mathscr{J} \underline{\mathcal{I}}_{G}\right)
$$

and

$$
i_{!}: \mathfrak{C a t}_{G}\left(\mathscr{J}, \underline{\mathcal{I}}_{G}\right) \rightarrow \mathfrak{C a t}_{G}\left(\mathscr{J}_{G}, \underline{\mathcal{I}}_{G}\right)
$$

given by restriction and left Kan extension along $i$ are inverse equivalences of enriched symmetric monoidal categories.

In other words the symmetric monoidal (topological) category $\mathcal{S}^{G}$ can simply be regarded as the symmetric monoidal (topological) category of objects in $\mathcal{S}$ equipped with a $G$-action.

The proof of Proposition A.19 requires a simple technical lemma ([53, Lemma V.1.1]).

Lemma A.20. Suppose that $V$ and $W$ are orthogonal $G$-representations with $\operatorname{dim} V=\operatorname{dim} W$. Then for any $U$

$$
O(V, U) \times O(V) \times O(W, V) \rightrightarrows O(V, U) \times O(W, V) \rightarrow O(W, U)
$$

is a (reflexive) coequalizer in $\mathcal{T}^{G}$.

Proof: Since the forgetful functor $\mathcal{T}^{G} \rightarrow \mathcal{T}$ preserves colimits and reflects isomorphisms, it suffices to prove the result in $\mathcal{T}$, where it is obvious, since the coequalizer diagram can be split by choosing an orthogonal (non-equivariant) isomorphism of $V$ with $W$.

Proof of Proposition A.19: Since $i: \mathscr{J} \rightarrow \mathscr{J}_{G}$ is fully faithful, the left Kan extension $i_{\text {! }}$ is fully faithful (see for example, [51, Corollary X.3]). To show that it is essentially surjective, let $W \in \mathscr{J}_{G}$ be any object, and let $V \in \mathscr{J}$ be a vector space of the same dimension as $W$. Define $X$ by the coequalizer

$$
(O(W, V) \times O(V))_{+} \wedge S^{-V} \rightrightarrows O(W, V)_{+} \wedge S^{-V} \rightarrow X .
$$

Since $\mathscr{J}_{G}(W, V)=O(W, V), i_{*} X$ is given by the coequalizer of

$$
\left(\mathscr{J}_{G}(W, V) \times \mathscr{J}_{G}(V, V)\right)_{+} \wedge S^{-V} \rightrightarrows \mathscr{J}_{G}(W, V)_{+} \wedge S^{-V} \rightarrow i_{*} X .
$$

There is thus a natural map

$$
i_{*} X \rightarrow S^{-W} .
$$

Evaluating at $U \in \mathscr{J}_{G}$ and using Lemma A.20 shows that (A.21) is an isomorphism. Thus $S^{-W}$ is in the image of $i_{*}$. It then follows easily that $i_{*}$ is essentially surjective.

Finally, the fact that $i_{*}$ is symmetric monoidal is immediate from the fact that left Kan extensions commute. It follows that $i^{*}$ is as well, since it is the inverse equivalence. 
A.2.7. Equivariant commutative and associative algebras. Using the notions described in $\S$ A.1.2 one can transport many algebraic structures to $\mathcal{S}^{G}$ using the symmetric monoidal smash product.

Definition A.22. A G-equivariant commutative (associative) algebra is a commutative (associative) algebra with unit in $\mathcal{S}^{G}$.

The conventions of $\S$ A.1.2 would dictate that we refer to the topological categories of $G$-equivariant commutative and associative algebras as comm $\mathcal{S}^{G}$ and ass $\mathcal{S}^{G}$. To ease some of the typesetting it will be convenient to employ the slightly abbreviated notation

$$
\begin{aligned}
\operatorname{Comm}^{G} & =\operatorname{comm} \mathcal{S}^{G} \\
\operatorname{Alg}^{G} & =\operatorname{ass} \mathcal{S}^{G},
\end{aligned}
$$

and to write $\mathbf{C o m m}_{G}$ and $\mathbf{A l g}_{G}$ for the corresponding $G$-equivariant topological categories of not necessarily equivariant algebra maps.

Since $\mathcal{S}^{G}$ is a closed symmetric monoidal category under $\wedge$, Proposition A.5 implies that both $\mathbf{C o m m}^{G}$ and $\mathbf{A l g}{ }^{G}$ are complete and cocomplete, and that the forgetful functors

$$
\begin{aligned}
\mathbf{C o m m}^{G} & \rightarrow \mathcal{S}^{G} \\
\mathbf{A l g}^{G} & \rightarrow \mathcal{S}^{G}
\end{aligned}
$$

create enriched limits, sifted colimits, and have left adjoints

$$
\begin{aligned}
\text { Sym }: \mathcal{S}^{G} & \rightarrow \mathbf{C o m m}^{G} \\
T: \mathcal{S}^{G} & \rightarrow \mathbf{A l g}^{G} .
\end{aligned}
$$

Similarly, there are categories of left and right modules over an associative alge$\operatorname{bra} A$. We will use the symbol $\mathcal{M}_{A}$ for the category of left $A$-modules. As described in $\S$ A.1.1, when $A$ is commutative, the category $\mathcal{M}_{A}$ inherits a symmetric monoidal product $M \bigwedge_{A} N$ defined by the reflexive coequalizer diagram

$$
M \wedge A \wedge N \rightrightarrows M \wedge N \rightarrow M \underset{A}{\wedge} N
$$

\section{A.3. Indexed monoidal products.}

A.3.1. Covering categories and fiberwise constructions. We begin with an example. Suppose that $(\mathcal{C}, \otimes, \mathbf{1})$ is a symmetric monoidal category and that $I$ is a finite set. Write $\mathcal{C}^{I}$ for the $I$-fold product of copies of $\mathcal{C}$. For notational purposes, and subsequent generalization it will be useful to think of an object of $\mathcal{C}^{I}$ as a functor $X: I \rightarrow \mathcal{C}$, with $I$ regarded as a category with no non-identity morphisms. The iterated monoidal product

$$
\otimes^{I} X=\bigotimes_{i \in I} X i
$$

defines a functor

$$
\otimes^{I}: \mathcal{C}^{I} \rightarrow \mathcal{C}
$$

The functor $\otimes^{I}$ is natural in isomorphisms in $I$ (this is just the symmetry of the symmetric monoidal structure). In this section we make use of the notion of a covering category to exploit this naturality in a systematic way.

Let $\mathfrak{S e t s}_{\text {iso }}$ be the groupoid of sets and isomorphisms. Suppose that $J$ is a category, and that $P: J \rightarrow \mathfrak{S e t s}_{\text {iso }}$ is a functor with the property that each $P j$ is 
finite. Then $P$ defines a $J$-diagram of finite sets, and the iterated monoidal product defines for each $j$ a functor

$$
\otimes^{P j}: \mathcal{C}^{P j} \rightarrow \mathcal{C}
$$

These vary functorially in $j$. This functoriality is expressed most cleanly using the Grothendieck construction [28, §VI.8] (see also [38, §B.1], or [50, p. 44] where the special case in which $\mathfrak{C a t}$ is replaced with $\mathfrak{S e t s}$ is attributed to Yoneda).

Suppose that $J$ is a category, and that $P: J \rightarrow \mathfrak{C}$ at is a functor. The Grothendieck construction associates to $P$ the category

$$
I=\int P
$$

of pairs $(j, s)$ with $j \in J$ and $s \in P(j)$. The set of maps from $(j, s)$ to $\left(j^{\prime}, s^{\prime}\right)$ is the set of pairs $(f, h)$ with $f: j \rightarrow j^{\prime}$ a map in $J$, and $h: P f(s) \rightarrow s^{\prime}$ a map in $P j^{\prime}$. By regarding a finite set as a category with no non-identity morphisms the Grothendieck construction also applies to functors $P: J \rightarrow \mathfrak{S e t s}_{\text {iso }}$.

A functor $p: I \rightarrow J$ arises from the Grothendieck construction of $P: J \rightarrow \mathfrak{S e t s}_{\text {iso }}$ if and only if it is satisfies the following two conditions

i) for every morphism $f: i \rightarrow j$ in $J$, and every $a \in I$ with $p a=i$, there is a unique morphism $g$ with domain $a$, and with $p g=f$;

ii) for every morphism $f: i \rightarrow j$ in $J$, and every $b \in I$ with $p b=j$, there is a unique morphism $g$ with range $b$, and with $p g=f$.

If $p: I \rightarrow J$ satisfies the above conditions, then $j \mapsto p^{-1}(j)$ defines a functor

$$
p^{-1}: J \rightarrow \mathfrak{S e t s}_{\text {iso }} .
$$

This structure is analogous to the notion of a covering space, and we name it accordingly.

Definition A.24. A functor $I \rightarrow J$ satisfying properties i) and ii) above is called a covering category.

A covering category $p: I \rightarrow J$ in which each of the fibers $p^{-1}(j)$ is finite will be called a finite covering category.

The aggregate of the functors (A.23) is a functor

$$
p_{*}^{\otimes}: \mathcal{C}^{I} \rightarrow \mathcal{C}^{J}
$$

given in terms of $p$ by

$$
p_{*}^{\otimes} X(j)=\bigotimes_{p(i)=j} X(i) .
$$

We will have much more to say about this in the next few sections. For now we focus on the general process that led to its construction.

Suppose we are given a formation of a category depending functorially on a set $I$, or in other words a functor

$$
C: \mathfrak{S e t s}_{\text {iso }} \rightarrow \mathfrak{C a t} .
$$

Given a covering category $p: I \rightarrow J$ let $C_{I} \rightarrow J$ be the category obtained by applying the Grothendieck construction to the composite

$$
J \rightarrow \mathfrak{S e t s}_{\text {iso }} \stackrel{C}{\rightarrow} \mathfrak{C a t}
$$


in which the first functor is the one classifying $I \rightarrow J$. Let $C(p)$ be the category of sections of $C_{I} \rightarrow J$. We will say that $C(p)$ is constructed from $C$ by working fiberwise. For example the category constructed from $C(S)=\mathcal{C}^{S}$ by working fiberwise is $\mathcal{C}^{I}$. The category constructed from the constant functor $C^{\prime}(S)=\mathcal{C}$ is $\mathcal{C}^{J}$.

A natural transformation $C \rightarrow C^{\prime}$ leads, via the same process, to a functor $C(p) \rightarrow C^{\prime}(p)$ which we will also describe as being constructed by working fiberwise.

A.3.2. Indexed monoidal products. When $(\mathcal{C}, \otimes, \mathbf{1})$ is a symmetric monoidal category, the diagram category $\mathcal{C}^{I}$ can be regarded as a symmetric monoidal category using the objectwise monoidal structure.

Definition A.25. Let $p: I \rightarrow J$ be a finite covering category and $(\mathcal{C}, \otimes, \mathbf{1})$ a symmetric monoidal category. The indexed monoidal product (along $p$ ) is the functor

$$
p_{*}^{\otimes}: \mathcal{C}^{I} \rightarrow \mathcal{C}^{J}
$$

constructed fiberwise from the iterated monoidal product.

For some purposes the notation $X^{\otimes(I / J)}$ is preferable to $p_{*}^{\otimes} X$. When $J$ is the one point $G$-set this can be further abbreviated to $X^{\otimes I}$. We use this alternate notation systematically when $\otimes$ is the smash product $\wedge$.

The properties of iterated monoidal products listed in the following proposition are straightforward.

Proposition A.26. The functor $\otimes^{I}: \mathcal{C}^{I} \rightarrow \mathcal{C}$ is symmetric monoidal. If

$$
\otimes: \mathcal{C}^{2} \rightarrow \mathcal{C}
$$

commutes with colimits in each variable then so does $\otimes^{I}$. In this situation $\otimes^{I}$ commutes with sifted colimits.

Applying Proposition A.26 fiberwise to a finite covering category $p: I \rightarrow J$ gives

Proposition A.27. The indexed monoidal product $p_{*}^{\otimes}: \mathcal{C}^{I} \rightarrow \mathcal{C}^{J}$ is symmetric monoidal. If

$$
\otimes: \mathcal{C}^{2} \rightarrow \mathcal{C}
$$

commutes with colimits in each variable then $p_{*}^{\otimes}$ commutes with sifted colimits.

Remark A.28. Though it plays no role in this paper, it can be useful to observe that the class of colimits preserved by $p_{*}^{\otimes}$ is slightly larger than the class of sifted colimits. For example $p_{*}^{\otimes}$ will commute with objectwise reflexive coequalizers, which are diagrams of the form

$$
X \rightrightarrows Y \rightarrow Z
$$

with the property that for each $j \in J$ there is a map $Y j \rightarrow X j$ completing

$$
X j \rightrightarrows Y j
$$

to a reflexive coequalizer diagram. The maps $Y j \rightarrow X j$ are not required to be natural in $j$.

The following is also straightforward

Proposition A.29. Suppose that $p: I \rightarrow J$ and $q: J \rightarrow K$ are covering categories. Then $q \circ p$ is a covering category, which is finite if $p$ and $q$ are. In that case there is a natural isomorphism

$$
q_{*}^{\otimes} \circ p_{*}^{\otimes} \approx(q \circ p)_{*}^{\otimes} .
$$


arising from the symmetric monoidal structure. This natural isomorphism is compatible with composition in the sense that if

$$
I \stackrel{p}{\rightarrow} J \stackrel{q}{\rightarrow} K \stackrel{r}{\rightarrow} L
$$

is a composition of finite covering categories categories, the diagram

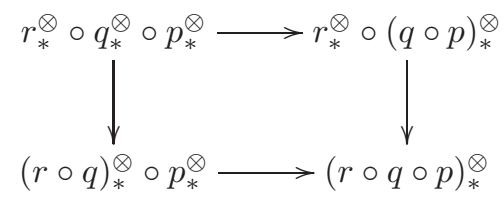

(in which the associativity isomorphisms have been suppressed) commutes.

The following results are also proved by working fiberwise.

Proposition A.30. Suppose that $\left(\mathcal{C}, \otimes, \mathbf{1}_{\mathcal{C}}\right)$ and $\left(\mathcal{D}, \wedge, \mathbf{1}_{\mathcal{D}}\right)$ are symmetric monoidal categories, and that

$$
\begin{gathered}
F: \mathcal{C} \rightarrow \mathcal{D} \\
T: F X \wedge F Y \rightarrow F(X \otimes Y) \\
\phi: \mathbf{1}_{\mathcal{D}} \rightarrow F \mathbf{1}_{\mathcal{D}}
\end{gathered}
$$

form a lax monoidal functor. If $p: I \rightarrow J$ is a finite covering category then $T$ gives a natural transformation

$$
p_{*}^{T}: p_{*}^{\wedge} \circ F^{I} \rightarrow F^{J} \circ p_{*}^{\otimes}
$$

between the two ways of going around

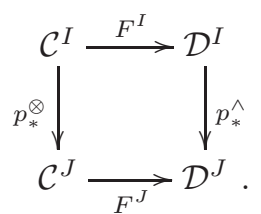

If $T$ is a natural isomorphism, then so is $p^{T}$.

The association $p \mapsto p_{*}^{T}$ of Proposition A.30 is compatible with the composition isomorphism of Proposition A.29 in the evident sense.

Suppose that $p: I \rightarrow J$ is a covering category, and $f: \tilde{J} \rightarrow J$ is a functor. Let $\tilde{I}$ be the "rigid pullback" category of pairs $\left(j^{\prime}, i\right) \in \tilde{J} \times I$ with $f\left(i^{\prime}\right)=p(j)$, and in which a morphism is a pair $\left(g, g^{\prime}\right)$ with $f(g)=p\left(g^{\prime}\right)$. Then the functor $\tilde{p}: \tilde{I} \rightarrow \tilde{J}$ defined by $\left(j^{\prime}, i\right) \mapsto j^{\prime}$ is a covering category.

Proposition A.31. In the situation described above, if $p: I \rightarrow J$ is finite then the following commutes up to a natural isomorphism given by the symmetric monoidal structure

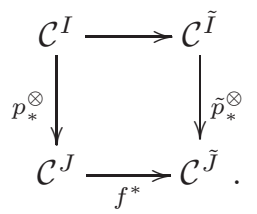


The categories $I$ and $J$ used in this paper arise from a left action of a group $G$ on a finite set $A$. Given such an $A$, let $\mathcal{B}_{A} G$ be the category whose set of objects is $A$ and in which a map $a \rightarrow a^{\prime}$ is an element $g \in G$ with the property that $g a=a^{\prime}$. When $A=$ pt we will abbreviate $\mathcal{B}_{A} G$ to just $\mathcal{B} G$. For any finite map $A \rightarrow B$ of $G$-sets, the corresponding functor

$$
\mathcal{B}_{A} G \rightarrow \mathcal{B}_{B} G
$$

is a covering category.

In the following series of examples we suppose $H \subset G$ is a subgroup, take $A=G / H$ to be the set of right $H$-cosets, and write $p: A \rightarrow \mathrm{pt}$ for the unique equivariant map. In this case the inclusion of the identity coset gives an equivalence

$$
\mathcal{B} H \rightarrow \mathcal{B}_{A} G
$$

and hence an equivalence of functor categories

$$
\mathcal{C}^{\mathcal{B}_{A} G} \rightarrow \mathcal{C}^{\mathcal{B} H} .
$$

An inverse is provided by the left Kan extension.

Example A.32. Suppose $\mathcal{C}$ is the category of abelian groups, with $\oplus$ as the symmetric monoidal structure. Then $\mathcal{C}^{\mathcal{B}_{A} G}$ is equivalent to the category of left $H$-modules, and the functor $p_{*}^{\oplus}$ is left additive induction. If the symmetric monoidal structure is taken to be the tensor product, then $p_{*}^{\otimes}$ is "norm induction."

Example A.33. Now take $(\mathcal{C}, \otimes, \mathbf{1})$ to be the category $\left(\mathcal{S}, \wedge, S^{0}\right)$ of orthogonal spectra. From the above and Proposition A.19, the category $\mathcal{S}^{\mathcal{B}_{A} G}$ is equivalent to the category of orthogonal $H$-spectra, and $\mathcal{S}^{\mathcal{B} G}$ is equivalent to the category of orthogonal $G$-spectra. In this case $p_{*}^{\wedge}$ defines a multiplicative transfer from orthogonal $H$-spectra to orthogonal $G$-spectra. This is the norm. It is discussed more fully in $\S A .4$ and $\S$ B.5.

Remark A.34. When $\mathcal{C}$ has all colimits and the tensor unit $\mathbf{1}$ is the initial object one may form infinite "weak" monoidal products, and the condition that $p: I \rightarrow J$ be finite may be dropped. If $I$ is an infinite set and $\{X i\}$ a collection of objects indexed by $i \in I$ set

$$
\otimes^{I} X i=\underset{I^{\prime} \subset I \text { finite }}{\lim _{\longrightarrow}} \otimes I^{I^{\prime}} X i
$$

in which the transition maps associated to $I^{\prime} \subset I^{\prime \prime}$ are given by tensoring with the unit

$$
\otimes^{I^{\prime}} X i \approx\left(\otimes^{I^{\prime}} X i\right) \otimes\left(\otimes^{I^{\prime \prime}-I^{\prime}} \mathbf{1}\right) \rightarrow \otimes^{I^{\prime \prime}} X i .
$$

The functor $p_{*}^{\otimes}$ is constructed by working fiberwise.

Remark A.35. The results of this section apply, with the obvious modifications, in the setting of enriched categories.

A.3.3. Distributive laws. Continuing with the same notation, we now assume that the category $\mathcal{C}$ comes equipped with two symmetric monoidal structures, $\otimes$ and $\oplus$, and that $\otimes$ distributes over $\oplus$ in the sense that there is a natural isomorphism

$$
A \otimes(B \oplus C) \approx(A \otimes B) \oplus(A \otimes C)
$$

compatible with all of the symmetries. For a precise definition see [45], or the definition of bipermutative category in [58, Chapter VI]. In all of our examples, $\oplus$ 
will be the categorical coproduct, and $A \otimes(-)$ will commute with all colimits. Given $p: I \rightarrow J$ and $q: J \rightarrow K$ we can form

$$
q_{*}^{\otimes} \circ p_{*}^{\oplus} .
$$

Our goal is to express this in the form

$$
q_{*}^{\otimes} \circ p_{*}^{\oplus}=r_{*}^{\oplus} \circ \pi_{*}^{\otimes} .
$$

We start with the case in which $K$ is the trivial category, and $p: I \rightarrow J$ is a map of finite sets. Let $\Gamma=\Gamma(I / J)$ be the set of sections $s: J \rightarrow I$ of $p$. Write ev : $J \times \Gamma \rightarrow I$ for the evaluation map, $\pi: J \times \Gamma \rightarrow \Gamma$ for the projection, and with an eye toward generalization, $r: \Gamma \rightarrow\{\mathrm{pt}\}$ for the unique map. The following lemma expresses the usual distributivity expansion

$$
\bigotimes_{j \in J}\left(\bigoplus_{p(i)=j} X i\right) \approx \bigoplus_{s \in \Gamma}\left(\bigotimes_{j \in J} X s(j)\right)
$$

in functorial terms.

Lemma A.36. The following diagram of functors commutes, up to a canonical natural isomorphism given by the symmetries of the symmetric monoidal structures

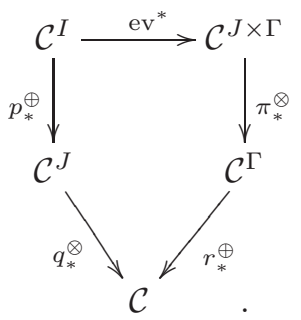

Working fiberwise, it is now a simple matter to deal with the more general case in which $p: I \rightarrow J$ and $q: J \rightarrow K$ are covering categories. Let $\Gamma$ be the category of pairs $(k, s)$, with $k \in K$ and $s$ a section of $(q \circ p)^{-1} k \rightarrow q^{-1} k$. A morphism $(k, s) \rightarrow\left(k, s^{\prime}\right)$ in $\Gamma$ is a map $f: k \rightarrow k^{\prime}$ making the following diagram commute

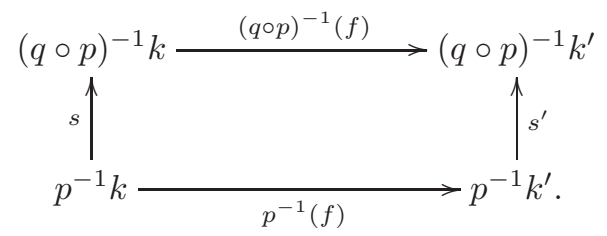

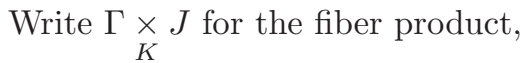

$$
\text { ev }: \underset{K}{\underset{\times}{\times}} J \rightarrow I
$$

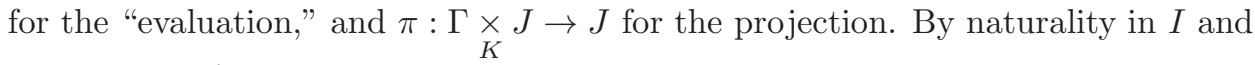
$J$ in Lemma A.36 we have 
Proposition A.37. The following diagram of functors commutes, up to a canonical natural isomorphism given by the symmetries of the symmetric monoidal structures

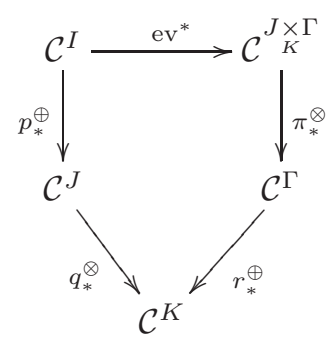

This formula is used in showing that the norm of a wedge of regular slice cells is a wedge of regular slice cells (Proposition 4.7), in the construction of monomial ideals (§A.3.6), and in describing the structure of equivariant twisted monoid rings and their monomial ideals $(\S 2.4)$.

A.3.4. Indexed monoidal products and pushouts. The homotopy theoretic properties of the norm depend on a formula for the indexed monoidal product of a pushout. We describe here the absolute case. The fiberwise analogue is spelled out in §B.8.2.

Suppose that $(\mathcal{C}, \otimes)$ is a closed symmetric monoidal category with finite colimits, and let $I$ be a finite set. For $X \in \mathcal{C}^{I}$ write $X^{\otimes I}$ for the iterated monoidal product. Suppose we are given a pushout diagram

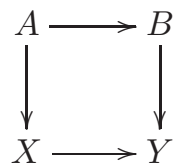

in $\mathcal{C}^{I}$. We wish to express $Y^{\otimes I}$ as an iterated pushout. Since the coequalizer diagram

$$
X \amalg A \amalg B \rightrightarrows X \amalg B \rightarrow Y
$$

can be completed to a reflexive coequalizer, the sequence

$$
(X \amalg A \amalg B)^{\otimes I} \rightrightarrows(X \amalg B)^{\otimes I} \rightarrow Y^{\otimes I}
$$

is a coequalizer (Proposition A.26). Using the distributivity law of $\S A .3 .3$ this can be re-written as

$$
\coprod_{I=I_{0} \amalg I_{1} \amalg I_{2}} X^{\otimes I_{0}} \otimes A^{\otimes I_{1}} \otimes B^{\otimes I_{2}} \rightrightarrows \coprod_{I=I_{0} \amalg I_{1}} X^{\otimes I_{0}} \otimes B^{\otimes I_{1}} \rightarrow Y^{\otimes I} .
$$

The horizontal arrows do not preserve the coproduct decompositions, but the sequence can be filtered by the cardinality of the exponent of $B$. Define fil ${ }_{n} Y$ by the coequalizer diagram

$$
\coprod_{\substack{I=I_{0} \amalg I_{1} \amalg I_{2} \\\left|I_{1}\right|+\left|I_{2}\right| \leq n}} X^{\otimes I_{0}} \otimes A^{\otimes I_{1}} \otimes B^{\otimes I_{2}} \rightrightarrows \coprod_{\substack{I=I_{0} \amalg I_{1} \\\left|I_{1}\right| \leq n}} X^{\otimes I_{0}} \otimes B^{\otimes I_{1}} \rightarrow \mathrm{fil}_{n} Y .
$$

Thus $\operatorname{fil}_{0} Y=X^{\otimes I}$ and $\operatorname{fil}_{|I|} Y=Y^{\otimes I}$. There is an evident coequalizer diagram

$$
\coprod_{\substack{I=I_{0} \amalg I_{1} \amalg I_{2} \\\left|I_{1}\right|+\left|I_{2}\right|=n}} X^{\otimes I_{0}} \otimes A^{\otimes I_{1}} \otimes B^{\otimes I_{2}} \rightrightarrows \operatorname{fil}_{n-1} Y \amalg\left(\coprod_{\substack{I=I_{0} \amalg I_{1} \\\left|I_{1}\right|=n}} X^{\otimes I_{0}} \otimes B^{\otimes I_{1}}\right) \rightarrow \operatorname{fil}_{n} Y,
$$


which can be re-written as a pushout square

$$
\begin{gathered}
\coprod_{\substack{I=I_{2} \amalg I_{1} \amalg I_{0} \\
\left|I_{0}\right|=|I|-n}} X^{\otimes I_{0}} \otimes A^{\otimes I_{1}} \otimes B^{\otimes I_{2}} \longrightarrow \coprod_{\substack{I=I_{1} \amalg I_{0} \\
\left|I_{1}\right|=n}} X^{\otimes I_{0}} \otimes B^{\otimes I_{1}} \\
\downarrow \\
\operatorname{fil}_{n-1} Y \longrightarrow \mathrm{fil}_{n} Y .
\end{gathered}
$$

The upper left term may be replaced by its effective quotient

$$
\coprod_{\left|I_{1}\right|=n} X^{\otimes I_{0}} \otimes \partial_{A} B^{\otimes I_{1}}
$$

in which $\partial_{A} B^{\otimes S}$ is defined by the coequalizer diagram

$$
\coprod_{\substack{S=S_{0} \amalg S_{1} \amalg S_{2} \\ S_{0} \neq \emptyset}} A^{\otimes S_{0}} \otimes A^{\otimes S_{1}} \otimes B^{\otimes S_{2}} \rightrightarrows \coprod_{\substack{S=S_{0} \amalg S_{1} \\ S_{0} \neq \emptyset}} A^{\otimes S_{0}} \otimes B^{\otimes S_{1}} \rightarrow \partial_{A} B^{\otimes S},
$$

leading to a pushout square

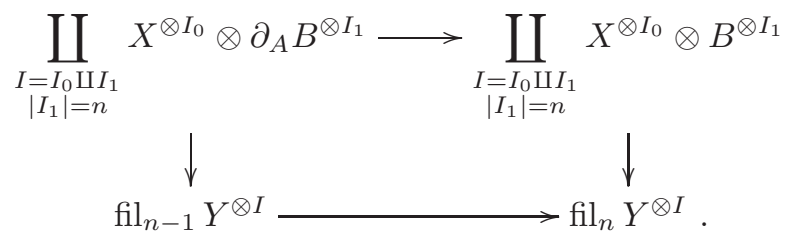

The object $\partial_{A} B^{\otimes S}$ can also be computed as the coequalizer of

$$
\coprod_{\substack{S=S_{0} \amalg S_{1} \amalg S_{2} \\\left|S_{0}\right|=\left|S_{1}\right|=1}} A^{\otimes S_{0}} \otimes A^{\otimes S_{1}} \otimes B^{\otimes S_{2}} \rightrightarrows \coprod_{\substack{S=S_{0} \amalg S_{1} \\\left|S_{0}\right|=1}} A^{\otimes S_{0}} \otimes B^{\otimes S_{1}} \rightarrow \partial_{A} B^{\otimes S},
$$

We call the map

$$
\partial_{A} B^{\otimes S} \rightarrow B^{\otimes S}
$$

the indexed corner map, since in the absolute case with $|I|=2$ it reduces to the "corner map" in

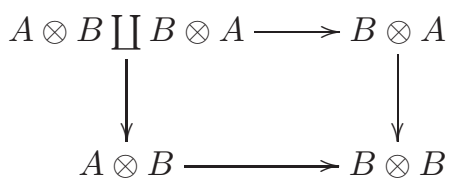

from the pushout of the top and left arrows to the bottom right term.

Remark A.42. The category of arrows in $\mathcal{C}$ becomes a closed symmetric monoidal category with

$$
\left(A_{1} \rightarrow B_{1}\right) \otimes\left(A_{2} \rightarrow B_{2}\right)
$$

taken to be the corner map in

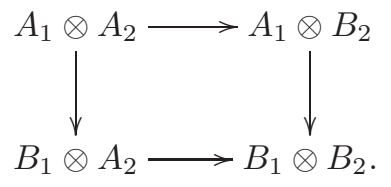

If $A \rightarrow B$ is a map in $\mathcal{C}^{S}$ then $(A \rightarrow B)^{\otimes S}$ works out to be $\partial_{A} B^{\otimes S} \rightarrow B^{\otimes S}$. 
By working fiberwise, one obtains a similar iterated pushout describing $p_{*}^{\otimes} Y$, involving the evident analogue $\partial_{A} p_{*}^{\otimes} B \rightarrow p_{*}^{\otimes} B$ of $\partial_{A} B^{\otimes S} \rightarrow B^{\otimes S}$.

Taking $A=X$ and $B=Y$ in (A.38) gives a filtration of the indexed monoidal product of any map. In the case of a pushout square (A.38) the two filtrations in fact coincide. We describe the absolute case. The relative case follows easily by working fiberwise.

Proposition A.43. Let

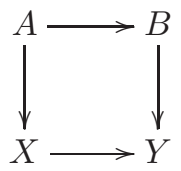

be a pushout square in $\mathcal{C}^{I}$.

i) The square

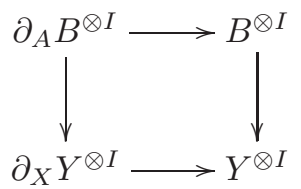

is a pushout square.

ii) The filtrations of $Y^{\otimes I}$ arising from (A.44) and

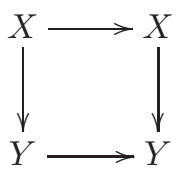

coincide.

Proof: The proof is by induction on $n=|I|$, the case in which $n=1$ being trivial. Let fil $_{m} Y^{\otimes I}$ be the filtration computed from the pushout square (A.44), and $\operatorname{fil}_{m}^{\prime} Y^{\otimes I}$ be the one computed from (A.45). The evident map of squares gives a natural map fil $m Y^{\otimes I} \rightarrow \operatorname{fil}_{m}^{\prime} Y^{\otimes I}$. Consider the diagram

$$
\begin{gathered}
\coprod_{\substack{I=I_{0} \amalg I_{1} \\
\left|I_{1}\right|=m}} X^{\otimes I_{0}} \otimes \partial_{A} B^{\otimes I_{1}} \longrightarrow \coprod_{\substack{I=I_{0} \amalg I_{1} \\
\left|I_{1}\right|=m}} X^{\otimes I_{0}} \otimes B^{\otimes I_{1}} \\
\coprod_{\substack{I=I_{0} \amalg I_{1} \\
\left|I_{1}\right|=m}} X^{\otimes I_{0}} \otimes \partial_{X} Y^{\otimes I_{1}} \longrightarrow \coprod_{\substack{I=I_{0} \amalg I_{1} \\
\left|I_{1}\right|=m}} X^{\otimes I_{0}} \otimes Y^{\otimes I_{1}} \\
\downarrow \\
\mathrm{fil}_{m-1} Y^{\otimes I} \underset{\mathrm{fil}_{m} Y^{\otimes I} .}{\downarrow}
\end{gathered}
$$

If $m<n$ then the induction hypothesis and part i) imply that the upper square is a pushout square. This shows that the map $\operatorname{fil}_{m} Y^{\otimes I} \rightarrow \mathrm{fil}_{m}^{\prime} Y^{\otimes I}$ is an isomorphism $m<n$. The case $m=n-1$ then gives an identification

$$
\text { fil }_{n-1} Y^{\otimes I}=\partial_{X} Y^{\otimes I},
$$


which, when combined with the pushout square

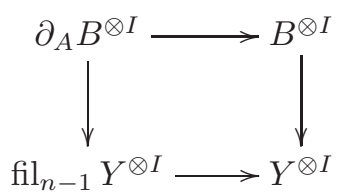

gives part i) for $I$.

By working in the category of arrows (as in Remark A.42) one can see that the formation of $\partial_{A}\left(p_{*}^{\otimes} B\right)$ is compatible with the isomorphism coming from the distributive law. More explicitly, let $I \stackrel{p}{\rightarrow} J \stackrel{q}{\rightarrow} K$ be a sequence of covering categories, and recall the basic diagram

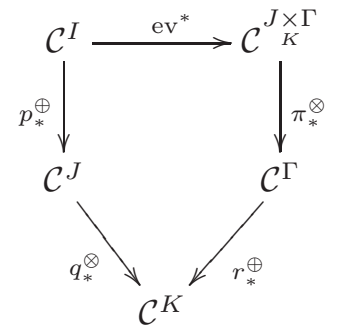

Suppose that $A \rightarrow B$ is a map in $\mathcal{C}^{I}$. The distributivity isomorphism in the arrow category is given by

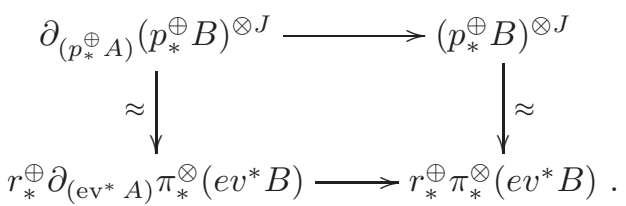

The fact that the left vertical arrow is an isomorphism is what expresses the compatibility of $\partial_{A} q_{*}^{\otimes} B$ with the distributive law.

A.3.5. Commutative algebras and indexed monoidal products. By Proposition A.5, if $\mathcal{C}$ is a co-complete closed symmetric monoidal category, then $\operatorname{comm} \mathcal{C}$ is cocomplete. The restriction functor $p^{*}: \operatorname{comm} \mathcal{C}^{J} \rightarrow \operatorname{comm} \mathcal{C}^{I}$ then has a left adjoint $p$ ! given by left Kan extension.

Proposition A.46. If $p: I \rightarrow J$ is a covering category, the following diagram commutes

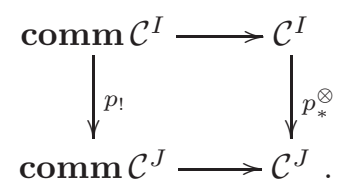

Proof: For a commutative algebra $A \in \operatorname{comm} \mathcal{C}^{I}$, and $j \in J$ the value of $p_{!} A$ at $j$ is calculated as the colimit over the category $I / j$ of the restriction of $p$. Since $p: I \rightarrow J$ is a covering category, the category $I / j$ is equivalent to the discrete category $p^{-1} j$, and so

$$
\left(p_{!} A\right) j=\otimes^{p^{-1} j} A,
$$


and the result follows.

A.3.6. Monomial ideals. Let $I$ be a set and consider the polynomial algebra

$$
A=\mathbb{Z}\left[x_{i}\right], \quad i \in I .
$$

As an abelian group, it has a basis consisting of the monomials $x^{f}$, with

$$
f: I \rightarrow\{0,1,2, \ldots\}
$$

a function taking the value zero on all but finitely many elements, and

$$
x^{f}=\prod_{j \in J} x_{j}^{f(j)} .
$$

The collection of such $f$ is a monoid under addition, and we denote it $\mathbb{N}_{0}^{I}$. If $D \subset \mathbb{N}_{0}^{I}$ is a monoid ideal then the subgroup $M_{D} \subset A$ with basis $\left\{x^{f} \mid f \in D\right\}$ is an ideal. These are the monomial ideals and they can be formed in any monoidal product of free associative algebras in any closed symmetric monoidal category.

Let $(\mathcal{C}, \otimes, \mathbf{1})$ be a closed symmetric monoidal category. Fix a set $I$ which we temporarily assume to be finite. Given $X \in \mathcal{C}^{I}$ let

$$
T X=\coprod_{n \geq 0} X^{\otimes n}
$$

be the free associative algebra generated by $X$. Write $A=p_{*}^{\otimes} T X \in \mathcal{C}$, where $p: I \rightarrow$ pt is the unique map. Then $A$ is an associative algebra in $\mathcal{C}$. The motivating example above occurs when $\mathcal{C}$ is the category of abelian groups and $X$ is the constant diagram $X i=\mathbb{Z}$.

Using Proposition A.37, the object $A$ can be expressed as an indexed coproduct

$$
A=\coprod_{f: I \rightarrow \mathbb{N}_{0}} X^{\otimes f}
$$

where $\mathbb{N}_{0}=\{0,1,2, \ldots\}$ and

$$
X^{\otimes f}=\bigotimes_{i \in I} X(i)^{\otimes f(i)} .
$$

The set

$$
\mathbb{N}_{0}^{I}=\left\{f: I \rightarrow \mathbb{N}_{0}\right\}
$$

is a commutative monoid under addition of functions. The multiplication map in $A$ is the sum of the isomorphisms

$$
X^{\otimes f} \otimes X^{\otimes g} \approx X^{\otimes(f+g)}
$$

given by the symmetry of the monoidal product $\otimes$, and the isomorphism

$$
X^{\otimes f(i)} \otimes X^{\otimes g(i)} \approx X^{\otimes(f(i)+g(i))} .
$$

For a monoid ideal $D \subset \mathbb{N}_{0}^{I}$, set

$$
M_{D}=\coprod_{f \in D} X^{\otimes f}
$$

The formula (A.47) for the multiplication in $A$ gives $M_{D}$ the structure of an ideal in $A$. If $D \subset D^{\prime}$ then the evident inclusion $M_{D} \subset M_{D^{\prime}}$ is an inclusion of ideals.

When $\mathcal{C}$ is pointed (in the sense that the initial object is isomorphic to the terminal object), the map

$$
A \rightarrow A / M_{D}
$$


is a map of associative algebras, where $A / M_{D}$ is defined by the pushout diagram

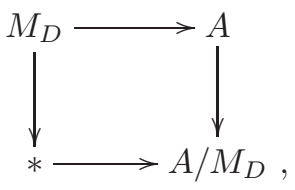

with $*$ denoting the terminal (and initial) object.

Definition A.48. The ideal $M_{D} \subset A$ is the monomial ideal associated to the monoid ideal $D$.

Example A.49. Suppose that $\operatorname{dim}: \mathbb{N}_{0}^{I} \rightarrow \mathbb{N}_{0}$ is any homomorphism. Given $d \in \mathbb{N}_{0}$ the set

$$
\{f \mid \operatorname{dim} f \geq d\}
$$

is a monoid ideal. We denote the corresponding monomial ideal $M_{d}$. The $M_{d}$ form a decreasing filtration

$$
\cdots \subset M_{d+1} \subset M_{d} \subset \cdots \subset M_{1} \subset M_{0}=A .
$$

When $\mathcal{C}$ is pointed, the quotient

$$
M_{d} / M_{d+1}
$$

is isomorphic as an $A$ bimodule to

$$
A / M_{1} \otimes \coprod_{\operatorname{dim} f=d} X^{\otimes f},
$$

in which $A$ act through its action on the left factor.

Remark A.50. The quotient module is defined by the pushout square

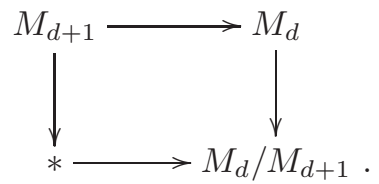

The pushout can be calculated in the category of left $A$-modules, $A$ bimodules, or just in $\mathcal{C}$.

Remark A.51. All of this discussion can be made to be covariant with respect to inclusion in $I$. Suppose that $I_{0} \subset I_{1}$ is an inclusion of finite sets and $X_{1}: I_{1} \rightarrow \mathcal{C}$ is an $I_{1}$-diagram. Define $X_{0}: I_{1} \rightarrow \mathcal{C}$ by

$$
X_{0}(i)= \begin{cases}X_{1}(i) & i \in I_{0} \\ * & \text { otherwise. }\end{cases}
$$

There is a natural map $X_{0} \rightarrow X_{1}$. Let $A_{0}$ and $A_{1}$ be the associative algebras constructed from the $X_{i}$ as described above. The algebra $A_{0}$ coincides with the one constructed directly from the restriction of $X_{0}$ to $I_{0}$. A monoid ideal $D_{1} \subset \mathbb{N}_{0}^{I_{1}}$ defines ideals $M_{D_{0}} \subset A_{0}$ and $M_{D_{1}} \subset A_{1}$. The monoid ideal $D_{0}$ is the same as the 
one constructed from the intersection of $D_{0}$ with $\mathbb{N}_{0}^{I_{0}}$, where $\mathbb{N}_{0}^{I_{0}}$ is regarded as a subset of $\mathbb{N}_{0}^{I_{1}}$ by extension by 0 . There is a commutative diagram

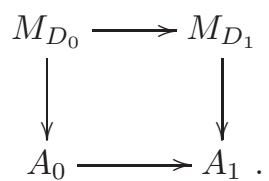

Using this, the construction of monomial ideals can be extended to the case of infinite sets $I$, by passing to the colimit over the finite subsets. As in the motivating example, when the set $I$ is infinite, the indexing monoid $\mathbb{N}_{0}^{I}$ is the set of finitely supported functions.

By working fiberwise, this entire discussion applies to the situation of a (possibly infinite) covering category $p: I \rightarrow J$. Associated to $X: I \rightarrow \mathcal{C}$ is

$$
A=p_{*}^{\otimes} T X \in \operatorname{ass} \mathcal{C}^{J}=(\operatorname{ass} \mathcal{C})^{J} .
$$

In case $I / J$ is infinite, the algebra $A$ is formed fiberwise by passing to the colimit from the finite monoidal products using the unit map, as described in Remark A.34. As an object of $\mathcal{C}^{J}$, the algebra $A$ decomposes into

$$
A=\coprod_{f \in \Gamma} X^{\otimes f}
$$

where $\Gamma$ is the set of sections of

$$
\mathbb{N}_{0}^{I / J} \rightarrow J
$$

with $\mathbb{N}_{0}^{I / J}$ formed from the Grothendieck construction applied to

$$
j \mapsto \mathbb{N}_{0}^{I_{j}} \quad\left(I_{j}=p^{-1}(j)\right) .
$$

The category $\mathbb{N}_{0}^{I / J}$ is a commutative monoid over $J$, and associated to any monoid ideal $D \subset \mathbb{N}_{0}^{I / J}$ over $J$, is a monomial ideal $M_{D} \subset A$.

The situation of interest in this paper (see $\S 2.4$ ) is when $I \rightarrow J$ is of the form

$$
\mathcal{B}_{K} G \rightarrow \mathcal{B} G
$$

associated to a $G$-set $K$, and the unique map $K \rightarrow$ pt. In this case $\mathbb{N}_{0}^{I / J}$ is the $G$-set $\mathbb{N}_{0}^{K}$ of finitely supported functions $K \rightarrow \mathbb{N}_{0}$. The relative monoid ideals are just the $G$-stable monoid ideals. A simple algebraic example arises in the case of a polynomial algebra $\mathbb{Z}\left[x_{i}\right]$ in which a group $G$ is acting on the set indexing the variables.

A.4. The norm. We now specialize the discussion of $\S$ A.3 to the case $(\mathcal{C}, \otimes, \mathbf{1})=$ $\left(\mathcal{S}, \wedge, S^{0}\right)$ and define the norm functor.

Because of Proposition A.19 we may identify the category of $G$-equivariant orthogonal spectra as the functor category $\mathcal{S}^{\mathcal{B} G}$. If $H \subset G$, then the functor

$$
i: \mathcal{B} H \rightarrow \mathcal{B}_{G / H} G
$$

sending the unique object to the coset $H / H$ is an equivalence of categories. This leads to an equivalence

$$
i^{*}: \mathcal{S}^{\mathcal{B}_{G / H} G} \rightarrow \mathcal{S}^{\mathcal{B} H},
$$

with inverse

$$
i_{!}: \mathcal{S}^{\mathcal{B} H} \rightarrow \mathcal{S}^{\mathcal{B}_{G / H} G}
$$


given by the left Kan extension. Write $p: \mathcal{B}_{G / H} G \rightarrow \mathcal{B} G$ for the functor corresponding to the $G$-map $G / H \rightarrow$ pt.

Definition A.52. The norm functor $N_{H}^{G}: \mathcal{S}^{H} \rightarrow \mathcal{S}^{G}$ is the composite

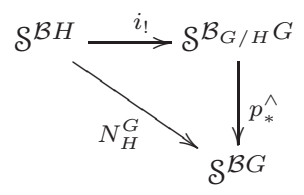

By Proposition A.27 we have

Proposition A.53. The functor $N_{H}^{G}$ is symmetric monoidal and commutes with sifted colimits.

Remark A.54. By Remark A.28, the norm also commutes with the formation of coequalizer diagrams in $\mathcal{S}^{H}$ whose underlying non-equivariant diagram in $\mathcal{S}$ extends to a reflexive coequalizer.

Remark A.55. We have defined the norm on the topological categories of equivariant spectra. Since it is symmetric monoidal it naturally extends to a functor of enriched categories

$$
N_{H}^{G}: \underline{\mathfrak{S}}_{H} \rightarrow \underline{\mathfrak{S}}_{G}
$$

compatible with the norm on spaces (and, in fact, spectra) in the sense that it gives for every $X, Y \in \underline{\mathcal{S}}_{H}$ a $G$-equivariant map

$$
N_{H}^{G}\left(\underline{\mathfrak{S}}_{H}(X, Y)\right) \rightarrow \underline{\mathfrak{S}}_{G}\left(N_{H}^{G} X, N_{H}^{G} Y\right) .
$$

By Proposition A.46, on equivariant commutative algebras the norm is the left adjoint of the restriction functor.

Corollary A.56. The following diagram commutes up to a natural isomorphism given by the symmetry of the smash product:

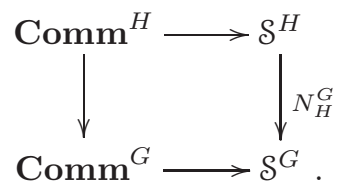

The left vertical arrow is the left adjoint to the restriction functor.

Remark A.57. Because of Corollary A.56 we will refer to the left adjoint to the restriction functor

$$
\mathrm{Comm}^{G} \rightarrow \mathrm{Comm}^{H}
$$

as the commutative algebra norm, and denote it

$$
N_{H}^{G}: \mathbf{C o m m}^{H} \rightarrow \mathbf{C o m m}^{G} .
$$

The Yoneda embedding gives a functor

$$
\begin{aligned}
\mathscr{J}^{o p} & \rightarrow \mathcal{S} \\
V & \mapsto S^{-V} .
\end{aligned}
$$


By definition of $\wedge$ this is a symmetric monoidal functor, and we are in the situation described in Proposition A.30. Thus if $p: I \rightarrow J$ is a covering category, there is a natural isomorphism between the two ways of going around

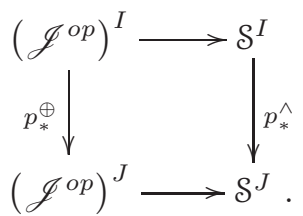

Take $I=\mathcal{B}_{G / H} G$ and $J=\mathcal{B} G$. Then the functor category $\left(\mathscr{J}^{o p}\right)^{I}$ is equivalent to the category $\left(\mathscr{J}^{H}\right)^{o p}$ (Proposition A.12), and $\mathcal{S}^{I}$ is equivalent to $\mathcal{S}^{H}$ (Proposition A.19). By naturality, the functor

$$
\mathscr{J}^{H^{o p}} \rightarrow \mathcal{S}^{H}
$$

corresponding to

$$
\left(\mathscr{J}^{o p}\right)^{I} \rightarrow \mathcal{S}^{I}
$$

is just the Yoneda embedding, and so sends an orthogonal $H$-representation $V$ to $S^{-V}$. Similarly $\left(\mathscr{J}^{o p}\right)^{J}$ is equivalent to $\mathscr{J}^{G^{o p}}, \mathcal{S}^{J}$ is equivalent to the category of orthogonal $G$-spectra, and the functor between them sends an orthogonal $G$ representation $W$ to $W^{-W}$. One easily checks (as in Example A.32) that the functor $p_{*}^{\oplus}$ corresponds to additive induction. We therefore have a commutative diagram

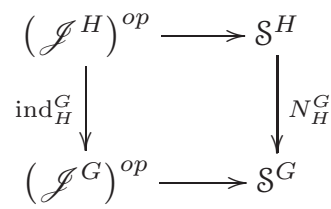

This proves

Proposition A.59. Let $V$ be a finite dimensional $H$-representation, and set $W=$ $\operatorname{ind}_{H}^{G} V$. There is a natural isomorphism

$$
N_{H}^{G} S^{-V} \approx S^{-W} .
$$

A.5. $h$-cofibrations. Suppose that $\mathcal{C}$ is a complete topological category (and in particular tensored and cotensored over $\mathcal{T}$ ).

Definition A.60. A map $i: A \rightarrow X$ in $\mathcal{C}$ is an $h$-cofibration if it has the homotopy extension property: given $f: X \rightarrow Y$ and a homotopy $h: A \otimes[0,1] \rightarrow Y$ with $\left.h\right|_{A \otimes\{0\}}=f \circ i$ there is an extension of $h$ to $H: X \otimes[0,1] \rightarrow Y$.

Example A.61. The mapping cylinder $A \rightarrow X \cup_{A} A \otimes[0,1]$ of any map $A \rightarrow X$ is an $h$-cofibration.

As is well-known, a map $i: A \rightarrow X$ is an $h$-cofibration if and only if

$$
\text { cyl } i=X \otimes\{0\} \underset{A \otimes\{0\}}{\cup} A \otimes[0,1] \rightarrow X \otimes[0,1]
$$

is the inclusion of a retract. 
Proposition A.62. The class of h-cofibrations is stable under composition, and the formation of coproducts and cobase change. Given a sequence

$$
X_{1} \stackrel{f_{1}}{\longrightarrow} \cdots \rightarrow X_{i} \stackrel{f_{i}}{\longrightarrow} X_{i+1} \rightarrow \cdots
$$

in which each $f_{i}$ is an h-cofibration, the map

$$
X_{1} \rightarrow \underset{i}{\lim } X_{i}
$$

is an h-cofibration.

Proposition A.63. Any topological functor $L$ which is a continuous left adjoint preserves the class of $h$-cofibrations.

Now suppose that $\mathcal{C}$ has a symmetric monoidal structure $\otimes$ which is compatible with the cartesian product of spaces, in the sense that for spaces $S$ and $T$, and objects $X, Y \in \mathcal{C}$ there is a natural isomorphism

$$
(X \otimes S) \otimes(Y \otimes T) \approx(X \otimes Y) \otimes(S \times T)
$$

compatible with the enrichment and the symmetric monoidal structures. Then given $i: A \rightarrow X$ we may form

$$
i^{\otimes n}: A^{\otimes n} \rightarrow X^{\otimes n}
$$

and regard it as a map in the category $\mathcal{C}^{\mathcal{B} \Sigma_{n}}$ of objects in $\mathcal{C}$ equipped with a $\Sigma_{n^{-}}$ action.

Proposition A.64. If $A \rightarrow X$ is an $h$-cofibration in $\mathcal{C}$, then for any $Z, A \otimes Z \rightarrow$ $X \otimes Z$ is an h-cofibration.

Proposition A.65. If $i: A \rightarrow X$ is an h-cofibration then $i^{\otimes n}$ is an $h$-cofibration in $\mathcal{C}^{\mathcal{B} \Sigma_{n}}$

Remark A.66. In the category of equivariant orthogonal spectra a version of this result appears in [54, Lemma 15.8] (where the reader is referred to [22, Lemma XII.2.3]).

Proof: The main point is to show that the diagonal inclusion

$$
\operatorname{cyl}\left(A^{\otimes n} \rightarrow X^{\otimes n}\right) \rightarrow \operatorname{cyl}(A \rightarrow X)^{\otimes n}
$$

is the inclusion of a $\Sigma_{n}$-equivariant retract. Granting this for the moment, one constructs a $\Sigma_{n}$-equivariant retraction of

$$
\operatorname{cyl}\left(A^{\otimes n} \rightarrow X^{\otimes n}\right) \rightarrow X^{\otimes n} \otimes[0,1]
$$

as the composition

$$
\begin{aligned}
X^{\otimes n} \otimes[0,1] \stackrel{1 \otimes \operatorname{diag}}{\longrightarrow} X^{\otimes n} \otimes[0,1]^{n} \approx( & X \otimes[0,1])^{\otimes n} \\
& \rightarrow \operatorname{cyl}(A \rightarrow X)^{\otimes n} \rightarrow \operatorname{cyl}\left(A^{\otimes n} \rightarrow X^{\otimes n}\right) .
\end{aligned}
$$

For the retraction of (A.67) start with the pushout square

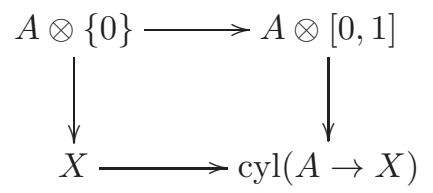


and consider the last stage of the filtration of $\operatorname{cyl}(A \rightarrow X)^{\otimes n}$ constructed in $\S A .3 .4$

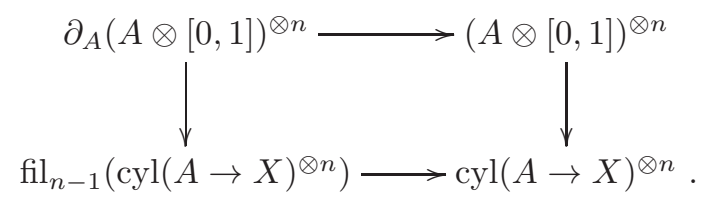

Form the $\Sigma_{n}$-equivariant map

$$
\operatorname{fil}_{n-1}\left(\operatorname{cyl}(A \rightarrow X)^{\otimes n}\right) \rightarrow X^{\otimes n} \rightarrow \operatorname{cyl}\left(A^{\otimes n} \rightarrow X^{\otimes n}\right)
$$

using the map $\operatorname{cyl}(A \rightarrow X) \rightarrow X$. To extend it to $\operatorname{fil}_{n}\left(\operatorname{cyl}(A \rightarrow X)^{\otimes n}\right)=\operatorname{cyl}(A \rightarrow$ $X)^{\otimes n}$ note that the top row of (A.68) can be identified with the tensor product of the identity map of $A^{\otimes n}$ with

$$
\partial_{\{0\}} I^{n} \rightarrow I^{n} .
$$

This identification is compatible with the action of the symmetric group. The desired extension is then constructed using any $\Sigma_{n}$-equivariant retraction of $I^{n}$ to the diagonal which takes $\partial_{\{0\}} I^{n}$ to $\{0\}$

Working fiberwise one concludes

Proposition A.69. Suppose that $\mathcal{C}$ is as above, and $p: I \rightarrow J$ is a covering category. The indexed monoidal product

$$
p_{*}^{\otimes}: \mathcal{C}^{I} \rightarrow \mathcal{C}^{J}
$$

preserves the class of h-cofibrations.

We end with a technical result which is useful for establishing some of the basic homotopy theoretic properties of equivariant orthogonal spectra, especially in connection with the monoidal geometric fixed point functor (§B.10). Though it does not appear explicitly in the literature in this form, it is a minor variation of [48, Appendix, Proposition 3.9] and is proved in the same manner.

Lemma A.70. An h-cofibration in $\mathcal{S}^{G}$ is an objectwise closed inclusion.

Proof: The assertion reduces immediately to showing that that $h$-cofibrations in the category of compactly generated weak Hausdorff spaces are closed inclusions. For this latter fact suppose that $A \subset X$ is an $h$-cofibration of compactly generated weak Hausdorff spaces. Then the mapping cylinder $\operatorname{cyl}(A \rightarrow X)$ is an equalizer of two maps from $X \times[0,1]$ to itself. Since $X \times[0,1]$ is weak Hausdorff, it is closed. But $A \subset X$ is the inverse image of $\operatorname{cyl}(A \rightarrow X)$ under the inclusion $X \times\{1\} \rightarrow X \times[0,1]$. See [48, Pages 488-9].

\section{ApPEndix B. Homotopy theORY of EQUiVARIANT ORTHOgOnAL SPECTRA}

We now turn to the stable homotopy theory of equivariant orthogonal spectra, the basis of which is the notion of stable weak equivalence defined in §2.2.4. Our goal is to set up the infrastructure needed for the proofs of properties $\mathbf{S p}_{\mathbf{1}}^{\mathbf{G}}-\mathbf{S p}_{6}^{\mathbf{G}}$, and for working with the formation of indexed wedges, smash products, symmetric powers and their compositions. These latter are explicit constructions, and to work with them in homotopy theory means determining, in each case, a full subcategory of $\mathcal{S}^{G}$ on which the construction preserves weak equivalences, and which is homotopically wide in the sense that it contains at least one object of each weak equivalence class, 
The standard way of doing this is to complete the set of weak equivalences to a Quillen model category structure, in such a way that each of the constructions takes weak equivalences between cofibrant objects to weak equivalences. This can be done in this case, but a problem arises when composing these operations. For example, in all of the standard model structures on $\mathcal{S}^{G}$, the symmetric powers of a cofibrant object are not cofibrant (or at least not known to be). The situation is reminiscent of the theory of unbounded operators, in which a domain of definition needs to be specified, and in which one can run into trouble trying to compose operators. It might be possible to find a model structure whose collection of cofibrant objects is preserved by all of these constructions. But this is more than is really required.

This is a situation where the language of model categories tends to obscure the basic task at hand. What is needed is to determine, for a given functor, a homotopically wide full subcategory on which the functor preserves weak equivalences. This problem depends only on the weak equivalences, and is most naturally considered in the context of homotopical categories. With this in mind we begin our work using homotopical categories, where the entire focus is on weak equivalences and derived functors, and put off introducing a model category structure until it is really needed.

Here is a summary of the contents of this appendix. In $\S B .1$ we review the theory of homotopical categories. Section B.2 introduces various notions of "flatness," which depend only on the class of weak equivalences, and play an important role in determining the homotopical properties of various functors. In $\S \mathrm{B} .3$ we develop a considerable amount of the stable homotopy theory of $\mathcal{S}^{G}$ using only the language of homotopical categories. This includes most of the results used in $\S 2.2 .5$ to verify $\mathbf{S p}_{1}^{\mathrm{G}}-\mathbf{S p}_{5}^{\mathrm{G}}$. Our analysis is facilitated by an approximation $\pi^{\mathrm{st}} \mathcal{S}^{G}$ to ho $\mathcal{S}^{G}$ which we study as a homotopical category in its own right. To go further it is helpful to have a model structure around and in $\S \mathrm{B} .4$ we define the positive complete model structure on $\mathcal{S}^{G}$. This is a variation on the positive stable model structure of [53] having the convenient property that indexed wedges and smash products of cofibrant objects are cofibrant. Sections B.5 and B.6 describe the homotopy properties of indexed smash and symmetric powers. Section B.7 contains a proof that the forgetful functor $\operatorname{comm} \mathcal{S}^{G} \rightarrow \mathcal{S}^{G}$ creates a model structure. The proofs of this that appear in the literature are incomplete, and it does not seem possible to give a complete proof without first analyzing the homotopy properties of indexed smash products. Section B.8 contains the important result that the formation of indexed smash products is homotopical on a subcategory of $\mathcal{S}^{G}$ containing both the cofibrant objects and the spectra underlying cofibrant commutative rings. This result is crucial for making use of the norm functor and is part of the reason that we work outside of the framework of model categories. Sections B.10 and B.11 contain results on the geometric fixed point functor and its interaction with the constructions described above. Finally, Section B.12 contains a construction of the real bordism spectrum $M U_{\mathbb{R}}$ on which all of the results of this paper are based.

B.1. Homotopical categories and model categories. We begin by reviewing some notions from [21].

Definition B.1. A homotopical category is a category $\mathcal{C}$ equipped with a class of morphisms called weak equivalences which contains all identity maps, and satisfies the two out of six property described below. 
The two out of six property asserts that in the situation

$$
\bullet \stackrel{u}{\rightarrow} \bullet \stackrel{v}{\rightarrow} \bullet \stackrel{w}{\rightarrow} \bullet
$$

if $v u$ and $w v$ are in $\mathcal{W}$ then so are $u, v, w$, and $v w u$. It implies the "two out of three" property (that two of three maps in composition being weak equivalences implies the third is), and that isomorphisms are weak equivalences.

Remark B.2. If the weak equivalences have the property that a map is a weak equivalence if and only if some functor applied to the map becomes an isomorphism, then identity maps are weak equivalences, the two out of six property automatically holds, and retracts of weak equivalences are weak equivalences.

Suppose that $\mathcal{C}$ is a homotopical category.

Definition B.3. A homotopy functor is a functor $F: \mathcal{C} \rightarrow \mathcal{D}$ with the property that $F(w)$ is an isomorphism whenever $w \in \mathcal{W}$.

There is a universal homotopy functor $L: \mathcal{C} \rightarrow$ ho $\mathcal{C}$ called the the localization of $\mathcal{C}$ with respect to $\mathcal{W}$. It is characterized uniquely up to unique isomorphism by the following universal property: for every category $\mathcal{D}$, and every homotopy functor $F: \mathcal{C} \rightarrow \mathcal{D}$ there is a unique functor ho $\mathcal{C} \rightarrow D$ making the diagram

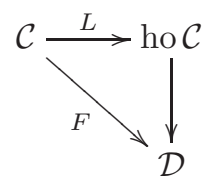

commute. While this characterization may seem stronger than is natural for characterizing an arrow in a 2-category, it simplifies the presentation. The difference between this and the 2-categorical formulation amounts to the convention that the map $\mathcal{C} \rightarrow$ ho $\mathcal{C}$ be the identity map on objects. The category ho $\mathcal{C}$ is the homotopy category of $\mathcal{C}$. Since the localization functor $L$ is the identity map on objects, it tends not to appear in notation.

Two issues emerge when working with homotopical categories. One is to find a description of ho $\mathcal{C}(X, Y)$ and the other is to describe conditions under which a functor $F: \mathcal{C} \rightarrow \mathcal{D}$ between homotopical categories induces a functor ho $F:$ ho $\mathcal{C} \rightarrow$ ho $\mathcal{D}$. For the first question the following can be helpful.

Proposition B.4. The transformation $\mathcal{C}(X,-) \rightarrow \operatorname{ho} \mathcal{C}(X,-)$ is the universal natural transformation from $\mathcal{C}(X,-)$ to a homotopy functor.

Proof: This is one situation where it is clearer to actually make use of the notation $L: \mathcal{C} \rightarrow$ ho $\mathcal{C}$. Spelled out, the assertion is that if $F: \mathcal{C} \rightarrow$ Sets is a homotopy functor and $\mathcal{C}(X,-) \rightarrow F$ a natural transformation, then there is a unique dotted arrow making the diagram

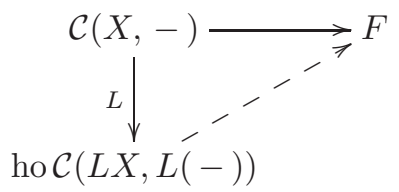

commute. Before describing the proof we make an observation about the property characterizing the functor $L: \mathcal{C} \rightarrow$ ho $\mathcal{C}$. For homotopy functors $F$ and $G$ on $\mathcal{C}$, this 
property supplies unique factorizations $F=\tilde{F} \circ L$ and $G=\tilde{G} \circ L$. It also implies that composition with $L$ gives a bijection between the set of natural transformations $\tilde{G} \rightarrow \tilde{F}$ and $G \rightarrow F$.

With this in mind we now turn to the proof of the proposition. By the Yoneda Lemma, the horizontal arrow in (B.5) is given by an element of $F(X)$. By the remark above, the set of natural transformations

$$
\text { ho } \mathcal{C}(L X, L(-)) \rightarrow F
$$

is in bijection with the set of natural transformations

$$
\text { ho } \mathcal{C}(L X,-) \rightarrow \tilde{F}
$$

which, again by Yoneda, is in one to one correspondence with the elements of $\tilde{F}(L X)=F(X)$. The map between these sets corresponding to the two ways of going around (B.5) is the identity.

Corollary B.6. Suppose that $\mathcal{C}$ is a homotopical category, and that $X \in \mathcal{C}$ has the property that $\mathcal{C}(X,-)$ is a homotopy functor. Then the natural transformation $\mathcal{C}(X,-) \rightarrow \operatorname{ho} \mathcal{C}(X,-)$ is a bijection.

Proof: Immediate from Proposition B.4.

For the second question, there is an apparatus of definitions to organize the situation.

Definition B.7. A functor between homotopical categories is homotopical if it sends weak equivalences to weak equivalences.

By the universal property, a homotopical functor $F: \mathcal{C} \rightarrow \mathcal{D}$ induces a functor ho $F:$ ho $\mathcal{C} \rightarrow$ ho $\mathcal{D}$. Furthermore, adjoint homotopical functors induce adjoint functors on the homotopy categories. But there are more general situations under which such a functor is induced. Suppose that $F: \mathcal{C} \rightarrow \mathcal{D}$ is a functor between homotopical categories and that one can find a subcategory $\mathcal{C}^{\prime} \subset \mathcal{C}$ on which $F$ is homotopical (where the weak equivalences in $\mathcal{C}^{\prime}$ are taken to be those morphisms which are weak equivalences in $\mathcal{C}$ ). Then $F$ induces a functor

$$
\text { ho } \mathcal{C}^{\prime} \rightarrow \text { ho } \mathcal{D} \text {. }
$$

If, in addition, ho $\mathcal{C}^{\prime} \rightarrow$ ho $\mathcal{C}$ is an equivalence of categories, then one gets an induced functor ho $\mathcal{C} \rightarrow$ ho $\mathcal{D}$ by composing with an inverse to this equivalence.

The situation becomes more manageable when there is a pair $(r, s)$ consisting of a functor $r: \mathcal{C} \rightarrow \mathcal{C}$ with the property that $F \circ r$ is homotopical, and a natural weak equivalence $s: r \rightarrow$ Id. In that case $\mathcal{C}^{\prime}$ can be taken to be the full subcategory generated by the image of $r$, the induced functor $\mathbf{L} F:$ ho $\mathcal{C} \rightarrow$ ho $\mathcal{D}$ can be computed as

$$
\mathbf{L} F X=F \circ r(X),
$$

and because of $s$, comes equipped with a natural transformation between the two ways of going around the diagram

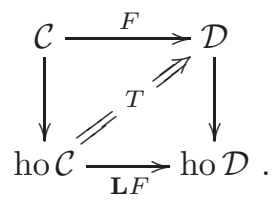


Together with this transformation, $\mathbf{L} F$ is characterized by a universal property. It is most easily stated if we overload some of the notation by using the symbol $F$ to denote the composite functor

$$
\mathcal{C} \stackrel{F}{\rightarrow} \mathcal{D} \rightarrow \text { ho } \mathcal{D}
$$

and identify functors ho $\mathcal{C} \rightarrow$ ho $\mathcal{D}$ with homotopy functors $\mathcal{C} \rightarrow$ ho $\mathcal{D}$. With these conventions we may regard the transformation $T$ as going from $\mathbf{L} F$ to $F$

$$
T: \mathbf{L} F \rightarrow F .
$$

The universal property is that if $G: \mathcal{C} \rightarrow$ ho $\mathcal{D}$ is a homotopy functor and $S: G \rightarrow F$ is a natural transformation, then there is a unique natural transformation $G \rightarrow \mathbf{L} F$ making

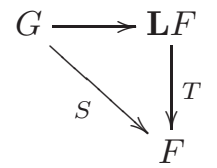

commute. Put differently, $\mathbf{L} F$ is the closest homotopy functor to the left of $F$.

The functor characterized by the above properties is the left derived functor of $F$. It is guaranteed to exist when $F$ is left deformable in the sense that there is a pair $(r, s)$ as above, and $F \circ r$ is homotopical.

A common situation arises when the weak equivalences on $\mathcal{C}$ refine to a model category structure, and $F$ takes weak equivalences between cofibrant objects to weak equivalences. In that case $F$ is left deformable, and one may take $(r, s)$ to be a functorial cofibrant replacement.

There are evident dual notions of a right deformable functors $F$ and a right derived functor $\mathbf{R} F$. For more on the definition of derived functors the reader is referred to [68] for the case of model categories, and to [21, Chapter VII] for the more general case of homotopical categories.

When

$$
F: \mathcal{C} \leftrightarrows \mathcal{D}: G
$$

are adjoint functors between homotopical categories, and $F$ is left deformable and $G$ is right deformable, then the derived functors

$$
\mathbf{L} F: \text { ho } \mathcal{C} \leftrightarrows \text { ho } \mathcal{D}: \mathbf{R} G
$$

are adjoint. See [21, Chapter VII, §44].

It is common, when there is no confusion likely, to drop the $\mathbf{L}$ from $\mathbf{L} F$ and not distinguish in notation between a functor and its derived functors. We follow this convention in the main body of the paper, where the emphasis is on homotopy theory.

B.2. Flat maps. The notion of a flat map and a flat functor was introduced in the unpublished manuscript [31] in order to isolate useful classes of maps and objects on which left derived functors can be computed. Though the original context involved model categories, the definitions involve only the weak equivalences and belong most naturally to the theory of homotopical categories. The dual notion was coined a "sharp map" by Charles Rezk, and used for a different purpose in [72].

Definition B.8. A functor $F: \mathcal{C} \rightarrow \mathcal{D}$ between categories with weak equivalences is flat if it is homotopical and preserves colimits. 
Typically the functor $F$ will be a left adjoint, and so will automatically preserve colimits.

Definition B.9. Suppose that $\mathcal{C}$ is a homotopical category possessing small colimits. A map $f: A \rightarrow X$ in $\mathcal{C}$ is flat if for every $A \rightarrow B$ and every weak equivalence $B \rightarrow B^{\prime}$, the map

$$
X \cup_{A} B \rightarrow X \cup_{A} B^{\prime}
$$

is a weak equivalence.

In other words a morphism $f$ is flat if and only if "cobase change along $f$ " preserves weak equivalences. Since cobase change is a left adjoint this is equivalent to the flatness of the cobase change functor.

Example B.10. A model category is left proper if and only if every cofibration is flat.

Proposition B.11. i) Finite coproducts of flat maps are flat.

ii) Composites of flat maps are flat

iii) Any cobase change of a flat map is flat.

iv) If a retract of a weak equivalence is a weak equivalence then a retract of a flat map is flat.

Proposition B.12. Suppose that

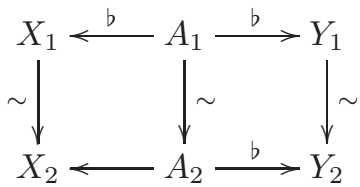

is a diagram in which $A_{2} \rightarrow Y_{2}$ and both maps in the top row are flat. If the vertical maps are weak equivalences, then so is the map

$$
X_{1} \underset{A_{1}}{\cup} Y_{1} \rightarrow X_{2} \underset{A_{2}}{\cup} Y_{2}
$$

of pushouts.

Proof: First suppose that $A_{1}=A_{2}=A$. Then

$$
X_{1} \cup_{A} Y_{1} \rightarrow X_{1} \cup_{A} Y_{2}
$$

is a weak equivalence since $A \rightarrow X_{1}$ is flat. The map $X_{1} \rightarrow X_{1} \cup Y_{A}$ is flat, since it is a cobase change of $A \stackrel{b}{\rightarrow} Y_{2}$ along $A \rightarrow X_{1}$. But this implies that

$$
X_{1} \cup_{A} Y_{2} \rightarrow X_{2} \cup_{X_{1}}\left(X_{1} \cup_{A} Y_{2}\right)=X_{2} \cup_{A} Y_{2} .
$$

is a weak equivalence. Putting these together gives the result in this case. 
For the general case, consider the following diagram

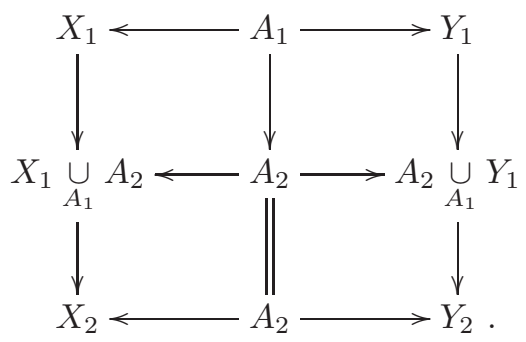

The flatness of the maps $A_{1} \rightarrow X_{1}$ and $A_{1} \rightarrow Y_{1}$ implies that the upper vertical maps (hence all the vertical maps) are weak equivalences, and that the maps in the middle row are flat. It also implies that

$$
A_{1} \rightarrow X_{1} \underset{A_{1}}{\cup} Y_{1}
$$

is flat. Since $A_{1} \rightarrow A_{2}$ is a weak equivalence, this means that

$$
X_{1} \underset{A_{1}}{\cup} Y_{1} \rightarrow A_{2} \underset{A_{1}}{\cup}\left(X_{1} \underset{A_{1}}{\cup} Y_{1}\right)
$$

is a weak equivalence. But this is the map from the pushout of the top row to the pushout of the middle row. By the case in which $A_{1}=A_{2}$, the map from the pushout of the middle row to the pushout of the bottom row is a also a weak equivalence. This completes the proof.

Remark B.13. If $\mathcal{C}$ has the property that every map can be factored into a flat map followed by a weak equivalence, then the above result holds with the assumption that only one of the maps in the top row is a weak equivalence. Suppose for instance that it is the map $A_{1} \rightarrow X_{1}$, and factor $A_{1} \rightarrow Y_{1}$ into a flat map $A_{1} \rightarrow Y_{1}^{\prime}$ followed by a weak equivalence $Y_{1}^{\prime} \rightarrow Y_{1}$ Now consider the diagram

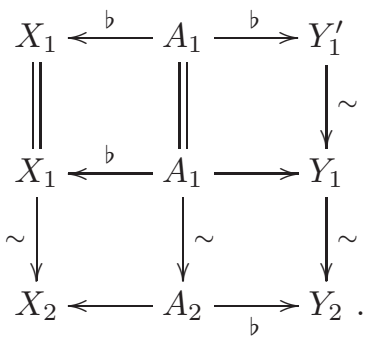

By Proposition B.12, the map from the pushout of the top row to the pushout of the middle row is a weak equivalence, as is the map from the pushout of the top row to the pushout of the bottom row. The map from the pushout of the middle row to the pushout of the bottom row is then a weak equivalence by the two out of three property of weak equivalences.

Remark B.14. In the category $\mathcal{S}^{G}$ equipped with the stable weak equivalences (B.3.1), the $h$-cofibrations will turn out to be flat. The mapping cylinder construction then factors every map into a flat map followed by a weak equivalence, so Remark B.13 applies. 
Now suppose that $(\mathcal{C}, \otimes, \mathbf{1})$ is a closed symmetric monoidal category, equipped with a class $\mathcal{W}$ of weak equivalences, making $\mathcal{C}$ into a homotopical category.

Definition B.15. An object $X \in \mathcal{C}$ is flat if the functor $X \otimes(-)$ is flat.

Showing that a symmetric monoidal structure on $\mathcal{C}$ induces one on ho $\mathcal{C}$ essentially comes down to exhibiting enough flat objects in $\mathcal{C}$. In $\S$ B.3.7 we will show that the cellular objects of $\mathcal{S}^{G}$ are flat.

Remark B.16. Suppose that every object $Z \in \mathcal{C}$ admits a weak equivalence equivalence $\tilde{Z} \rightarrow Z$ from a flat object $\tilde{Z}$. If $X \rightarrow Y$ is a weak equivalence of flat objects, so is $X \otimes Z \rightarrow Y \otimes Z$ for any $Z$. This follows from the diagram

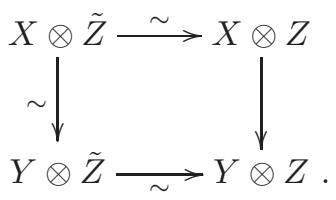

B.3. Equivariant stable homotopy theory. The weak equivalences were defined in $§ 2.2 .4$ as the maps inducing isomorphisms of stable homotopy groups. Equipped with them $\mathcal{S}^{G}$ becomes a homotopical category, and the functor $\mathcal{S}^{G} \rightarrow$ ho $\mathcal{S}^{G}$ is defined. In this section we establish many of the basic properties of ho $\mathcal{S}^{G}$, including most of the results used in $\S 2.2 .5$ to verify $\mathbf{S p}_{\mathbf{1}}^{\mathbf{G}}-\mathbf{S p}_{\mathbf{5}}^{\mathbf{G}}$ of $\S 2.2 .1$.

B.3.1. Stable weak equivalences and basic homotopical functors. We begin with some basic homotopical functors.

Proposition B.17. The formation of filtered colimits along objectwise closed inclusions is homotopical.

Proof: This is immediate from the fact that formation of homotopy groups commutes with filtered colimits of closed inclusions.

Since $h$-cofibrations are objectwise closed inclusions (Lemma A.70), Proposition B.17 applies to the formation of filtered colimits along $h$-cofibrations.

The following three results, which are part of [53, Theorem III.3.5] (see also [54, Theorem 7.4 iv)]), imply that many basic functors are homotopical.

Proposition B.18. Suppose $f: X \rightarrow Y$ is a map and let $F \rightarrow X$ be the homotopy fiber, defined by the pullback square

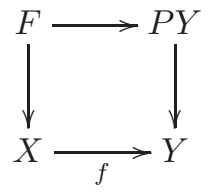

in which $P Y$ is the path spectrum of $Y$. There is a long exact sequence

$$
\cdots \rightarrow \pi_{k}^{H} F \rightarrow \pi_{k}^{H} X \rightarrow \pi_{k}^{H} Y \rightarrow \pi_{k-1}^{H} F \rightarrow \cdots
$$

Sketch of proof: This sequence is gotten by passing to the colimit from the exact sequence

$$
\cdots \rightarrow \pi_{k+V}^{H} F_{V} \rightarrow \pi_{k+V}^{H} X_{V} \rightarrow \pi_{k+V}^{H} Y_{V} \rightarrow \pi_{k-1+V}^{H} F_{V} \rightarrow \cdots .
$$


Proposition B.19. For any $X$, any $H \subset G$, and any $k \in \mathbb{Z}$ the suspension map

$$
\pi_{k}^{H} X \rightarrow \pi_{k+1}^{H} S^{1} \wedge X
$$

is an isomorphism.

Sketch of proof: Choose an exhausting sequence $\left\{V_{n}\right\}$ with the property that $V_{n} \oplus \mathbb{R} \subset V_{n+1}$. Then the map $\pi_{k+V_{n}}^{H} X_{V_{n}} \rightarrow \pi_{k+V_{n+1}}^{H} X_{V_{n+1}}$ factors through the suspension map $\pi_{k+1+V_{n}}^{H} S^{1} \wedge X_{V_{n}}$, and so the sequence for computing $\pi_{k+1}^{H} S^{1} \wedge X$ threads through the sequence for computing $\pi_{k}^{H} X$.

Proposition B.20. Let $X \rightarrow Y$ be an h-cofibration.

i) The map $Y \cup C X \rightarrow Y / X$ is a weak equivalence.

ii) There is a natural long exact sequence of stable homotopy groups

$$
\cdots \rightarrow \pi_{k}^{H} X \rightarrow \pi_{k}^{H} Y \rightarrow \pi_{k}^{H}(Y / X) \rightarrow \pi_{k-1}^{H} X \rightarrow \ldots,
$$

in which the map $\pi_{k} Y \rightarrow \pi_{k} Y / X$ is induced by the evident quotient map, and the connecting homomorphism $\pi_{k}^{H} Y / X \rightarrow \pi_{k-1}^{H} X$ is induced by the maps

$$
Y / X \leftarrow Y \cup C X \rightarrow \Sigma X .
$$

and the suspension isomorphism of Proposition B.19.

Sketch of proof: For the first part, since $A \rightarrow X$ is an $h$-cofibration, the map $X \cup C A \rightarrow X / A$ is a homotopy equivalence, hence induces an isomorphism of stable homotopy groups. The result can then be deduced from Proposition B.19 as in [48, III.2.1].

Corollary B.21. The h-cofibrations in $\mathcal{S}^{G}$ are flat.

Proposition B.20 implies that the formation of mapping cones is homotopical as is the formation of quotients of $h$-cofibrations. It also gives parts i) and iii) of the Proposition below. Part ii) follows from the fact that the formation of unstable homotopy groups commutes with products and the fact that filtered colimits commute with finite products.

Proposition B.22. i) For any any set of spectra $\left\{X_{\alpha}\right\}$ the map

$$
\bigoplus \pi_{*}^{G} X_{\alpha} \rightarrow \pi_{*}^{G} \bigvee X_{\alpha}
$$

is an isomorphism, hence the formation of wedges is homotopical.

ii) For any any finite set of spectra $\left\{X_{\alpha}\right\}$ the map

$$
\pi_{*}^{G} \prod X_{\alpha} \rightarrow \prod \pi_{*}^{G} X_{\alpha}
$$

is an isomorphism, hence the formation of finite products is homotopical.

iii) For any finite set of spectra $\left\{X_{\alpha}\right\}$ the map

$$
\bigvee X_{\alpha} \rightarrow \prod X_{\alpha}
$$

is a weak equivalence. 
Corollary B.23. The category ho $\mathcal{S}^{G}$ is additive, and admits finite products and arbitrary coproducts. The coproducts are given by wedges and the finite products by finite products.

Proof: Let's begin with the case of coproducts. Let $J$ be a set. The adjoint functors

$$
\bigvee:\left(\mathcal{S}^{G}\right)^{J} \leftrightarrows \mathcal{S}^{G}: \operatorname{diag}
$$

are homotopical by Proposition B.22. They therefore induce adjoint functors

$$
\bigvee:\left(\operatorname{ho} \mathcal{S}^{G}\right)^{J} \leftrightarrows \operatorname{hoS}^{G}: \operatorname{diag}
$$

on the homotopy categories. This shows that arbitrary coproducts exist in ho $\mathcal{S}^{G}$ and that they may be computed as wedges. A similar argument shows that finite products exist, are computed as products in $\mathcal{S}^{G}$, and that the map from a finite coproduct to a finite product is an isomorphism. This endows the morphism sets in ho $\mathcal{S}^{G}$ with the structure of commutative monoids. That they are in fact abelian groups can be seen by checking that for all $X$, the "shearing map" $X \vee X \rightarrow X \times X$, with first component the projection to the first summand and second component the coproduct of the identity map with itself, is a weak equivalence.

The "indexed" analogue of Proposition B.22 is also true, and appears as Proposition B.56. It expresses a kind of "equivariant additivity" on ho $\mathcal{S}^{G}$.

B.3.2. Suspension and zero space. The suspension and zero space functors were defined in Definition 2.7. Formation of the suspension spectrum is nearly homotopical.

Proposition B.24. The suspension spectrum functor is homotopical on the subcategory of non-degenerately based $G$-spaces. The right derived functor $\mathbf{R} \Omega^{\infty} X$ may be computed as

$$
\mathbf{R} \Omega^{\infty} X=\text { ho } \underset{\lim }{\longrightarrow} \Omega^{V_{n}} X_{V_{n}}
$$

where $\left\{V_{n}\right\}$ is any choice of exhausting sequence, and $\Omega^{V_{n}}(-)$ is the $G$-space of non-equivariant maps.

Proof: The assertion about suspension spectra follows from the fact that if $K \rightarrow L$ is an equivariant weak equivalence of non-degenerately based $G$-spaces, then so is

$$
S^{V} \wedge K \rightarrow S^{V} \wedge L
$$

for any representation $V$. This reduces to the statement that for every $H \subset G$, the map

$$
S^{V^{H}} \wedge K^{H} \rightarrow S^{V^{H}} \wedge L^{H}
$$

is a weak equivalence, assuming $K^{H} \rightarrow L^{H}$ is. But this is a standard fact. The functor $\mathbf{R} \Omega^{\infty} X=\operatorname{holim} \Omega^{V_{n}} X_{V_{n}}$ is clearly homotopical, so what is needed for the second assertion is to construct a functorial weak equivalence $X \rightarrow X^{\prime}$, in which $X^{\prime}$ has the property that the map

$$
\mathbf{R} \Omega^{\infty} X^{\prime} \rightarrow \Omega^{\infty} X^{\prime}
$$

is a weak equivalence. One way to do this is to define $X \rightarrow X^{\prime}$ by $X_{V} \rightarrow$ holimn $\Omega^{V_{n}} X_{V \oplus V_{n}}=X_{V}^{\prime}$. (One can also take $X^{\prime}$ to be the functorial fibrant 
replacement coming from the small object construction in the positive complete model structure of $\S B$.4.1.)

Adding a "whisker" provides a left deformation to $\Sigma^{\infty}$, and the natural transformation $X \rightarrow X^{\prime}$ appearing in the proof above gives a right deformation of $\Omega^{\infty}$. The derived suspension spectrum and zero space functors therefore induce adjoint functors on the homotopy categories

$$
\mathbf{L} \Sigma^{\infty}: \text { ho } \mathcal{T}^{G} \leftrightarrows \operatorname{ho}^{G}: \mathbf{R} \Omega^{\infty}
$$

B.3.3. An approximation to the homotopy category. Our further analysis of ho $\mathcal{S}^{G}$ is facilitated by an approximation, $\pi^{\text {st }} \mathcal{S}^{G}$.

Let

$$
\varepsilon_{V}: S^{-V} \wedge S^{V} \rightarrow S^{0}
$$

be the map adjoint to the identity map of $S^{V}$. Associated to a linear isometric embedding $t: V \rightarrow W$ is a map

$$
S^{-W} \wedge S^{W} \rightarrow S^{-V} \wedge S^{V}
$$

One way to describe it is to note that the space of such maps is the space of equivariant maps

$$
S^{W} \rightarrow\left(S^{-V} \wedge S^{V}\right)_{W}
$$

and that

$$
\left(S^{-V} \wedge S^{V}\right)_{W} \approx \operatorname{Thom}(O(V, W) ;(W-V) \oplus V) \approx O(V, W)_{+} \wedge S^{W} .
$$

The map (B.26) corresponds to smashing the identity map of $S^{W}$ with the map $S^{0} \rightarrow O(V, W)_{+}^{G}$ sending the non-base point to $t$. The map (B.26) can also be expressed as $\operatorname{Id} \wedge \epsilon_{U}$ after rewriting the domain as

$$
S^{-V} \wedge S^{V} \wedge S^{-U} \wedge S^{U}
$$

with $U=W-t(V)$. When $V<W$ the fixed point space $O(V, W)^{G}$ is connected, and so the homotopy class (B.26) is independent of the choice of $t$.

For $X, Y \in \mathcal{S}^{G}$ let

$$
\pi^{\mathrm{st}} \mathcal{S}^{G}(X, Y)=\underset{V}{\lim } \pi_{0} \mathcal{S}^{G}\left(S^{-V} \wedge S^{V} \wedge X, Y\right),
$$

in which the limit is taken over the partially ordered set of representations of $G$ $(\S 2.2 .4)$. We wish to make $\pi^{\text {st }} \mathcal{S}^{G}(X, Y)$ into the morphisms in a category. For this we need to define the composition law. An element $f \in \pi^{\text {st }} \mathcal{S}^{G}$ is represented by a map $f_{V}: S^{-V} \wedge S^{V} \wedge X \rightarrow Y$. Given $f \in \pi^{\text {st } \mathcal{S}^{G}}(X, Y)$ and $g \in \pi^{\text {st }} \mathcal{S}^{G}(Y, Z)$ represented by

$$
\begin{gathered}
f_{V}: S^{-V} \wedge S^{V} \wedge X \rightarrow Y \\
g_{W}: S^{-W} \wedge S^{W} \wedge Y \rightarrow Z
\end{gathered}
$$

the composition $g \circ f$ is defined to be the equivalence class of the map

$$
(g \circ f)_{W \oplus V}: S^{-W \oplus V} \wedge S^{W \oplus V} \wedge X \rightarrow Z
$$

constructed from the isomorphism

$$
S^{-W \oplus V} \wedge S^{W \oplus V} \approx S^{-W} \wedge S^{W} \wedge S^{-V} \wedge S^{V}
$$

and the composite

$$
S^{-W} \wedge S^{W} \wedge S^{-V} \wedge S^{V} \wedge X \stackrel{\mathrm{Id} \wedge f_{V}}{\longrightarrow} S^{-W} \wedge S^{W} \wedge Y \stackrel{g_{W}}{\longrightarrow} Z .
$$


Associativity of the composition follows from the associativity of the smash product.

Definition B.27. The category $\pi^{\text {st }} \mathcal{S}^{G}$ is the category whose objects are those of $\mathcal{S}^{G}$, with morphisms $\pi^{\text {st }} \mathcal{S}^{G}(X, Y)$, and the composition law described above.

One thing that makes $\pi^{\text {st }} \mathcal{S}^{G}$ so useful is that the hom sets are easy to describe, and yet the functors $\pi_{k}^{H}$ factor through it and are corepresentable.

Proposition B.28. For all $k \in \mathbb{Z}$, there is a natural isomorphism

$$
\pi^{s t} \mathcal{S}^{G}\left(G / H_{+} \wedge S^{k}, Y\right) \approx \pi_{k}^{H}(Y) .
$$

Proof: Suppose $k \geq 0$. Then

$$
\begin{aligned}
& \pi^{\mathrm{st}} \mathcal{S}^{G}\left(G / H_{+} \wedge S^{k}, Y\right)=\underset{\lim }{\longrightarrow} \pi_{0} \mathcal{S}^{G}\left(S^{-V} \wedge S^{V} \wedge G / H_{+} \wedge S^{k}, Y\right) \\
& =\underline{\lim } \pi_{0} \mathcal{S}^{H}\left(S^{-V} \wedge S^{V} \wedge S^{k}, Y\right) \\
& =\lim _{\longrightarrow} \pi_{0} \mathcal{T}^{H}\left(S^{V} \wedge S^{k}, Y_{V}\right) \\
& =\lim _{k+V} \pi_{V}^{H} Y_{V}=\pi_{k}^{H} Y \text {. }
\end{aligned}
$$

Similarly,

$$
\begin{aligned}
\pi^{\mathrm{st}} \mathcal{S}^{G}\left(G / H_{+} \wedge S^{-k}, Y\right) & =\underset{\longrightarrow}{\longrightarrow} \pi_{0} \mathcal{S}^{G}\left(S^{-V} \wedge S^{V} \wedge G / H_{+} \wedge S^{-k}, Y\right) \\
& =\underset{\lim }{\longrightarrow} \pi_{0} \mathcal{S}^{H}\left(S^{-V} \wedge S^{V} \wedge S^{-k}, Y\right) \\
& =\underset{\mathrm{lim}}{\longrightarrow} \pi_{0} \mathcal{T}^{H}\left(S^{V}, Y_{V+k}\right) \\
& =\underset{\mathrm{l}}{\longrightarrow} \pi_{V}^{H} Y_{V+k}=\underset{W>k}{\lim _{W-k}} \pi_{W}^{H} Y_{W}=\pi_{-k}^{H} Y .
\end{aligned}
$$

Proposition B.28 implies that a map $X \rightarrow Y \in \mathcal{S}^{G}$ which becomes an isomorphism in $\pi^{\text {st } \mathcal{S}^{G}}$ is a weak equivalence. An important example is

Proposition B.30. Suppose that $V$ is a representation of $G$. For every $X$, the map

$$
S^{-V} \wedge S^{V} \wedge X \rightarrow X
$$

is an isomorphism in $\pi^{s t} \mathcal{S}^{G}$, hence a weak equivalence.

Proof: We will show that for all $Y$, the map

$$
\pi^{\mathrm{st}} \mathcal{S}^{G}(X, Y) \rightarrow \pi^{\mathrm{st}} \mathcal{S}^{G}\left(S^{-V} \wedge S^{V} \wedge X, Y\right)
$$

is an isomorphism. By definition,

$$
\pi^{\mathrm{st}} \mathcal{S}^{G}(X, Y)=\underset{W}{\lim }\left(S^{-W} \wedge S^{W} \wedge X, Y\right),
$$

while

$$
\pi^{\text {st }} \mathcal{S}^{G}\left(S^{-V} \wedge S^{V} \wedge X, Y\right)=\underset{U}{\lim } \pi_{0} \mathcal{S}^{G}\left(S^{-U} \wedge S^{U} \wedge S^{-V} \wedge S^{V} \wedge X, Y\right) .
$$

Writing $W=U \oplus V$ and using the identification $S^{-W} \wedge S^{W} \approx S^{-U} \wedge S^{U} \wedge S^{-V} \wedge S^{V}$, this last colimit may be replaced by

$$
\underset{W>V}{\lim _{W}} \pi_{0} \mathcal{S}^{G}\left(S^{-W} \wedge S^{W} \wedge X, Y\right),
$$


since the set $\{U \mid U \oplus V>V\}$ is cofinal in the poset of all representations. But this clearly coincides with (B.32), since $\{W \mid W>V\}$ is also cofinal in the poset of representations.

Remark B.33. The weak equivalence (B.31) is often written in the form

$$
S^{-V \oplus W} \wedge S^{W} \wedge X \rightarrow S^{-V} \wedge X .
$$

This is gotten from (B.31) by writing $S^{-V \oplus W}$ as $S^{-V} \wedge S^{-W}$ and writing the map as

$$
S^{-W} \wedge S^{W} \wedge\left(S^{-V} \wedge X\right) \rightarrow\left(S^{-V} \wedge X\right) .
$$

Corollary B.34. Suppose that $V$ is a representation of $G$. Smashing with $S^{V}$ and $S^{-V}$ are inverse equivalences of $\pi^{s t} \mathcal{S}^{G}$.

Remark B.35. Corollary B.34 does not directly imply the analogous statement for ho $\mathcal{S}^{G}$. For that one needs to know that smashing with $S^{V}$ and $S^{-V}$ are homotopical. This will be proved in $\S B .3 .5$.

One consequence of Corollary B.34 is that $\pi^{\text {st }} \mathcal{S}^{G}$ is tensored over the equivariant Spanier-Whitehead category $\mathcal{S} \mathcal{W}^{G}$ defined in $\S 2.2 .1$. The main point is to show that

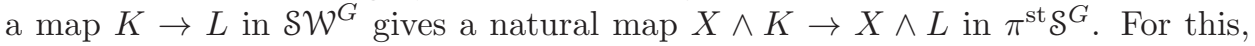
suppose that the map $K \rightarrow L$ is represented by a map of spaces $S^{V} \wedge K \rightarrow S^{V} \wedge L$. This latter map gives us an element of

$$
\pi^{\mathrm{st}} \mathcal{S}^{G}\left(X \wedge S^{V} \wedge K, X \wedge S^{V} \wedge L\right)
$$

and hence an element of $\pi^{\text {st } \mathcal{S}^{G}}(X \wedge K, Y \wedge L)$ under the isomorphism of Corollary B.34.

This fact leads to a form of Spanier-Whitehead duality in $\pi^{\text {st }} \mathcal{S}^{G}$. Suppose that $K$ is a finite $G$-CW complex, and that $L$ is a " $V$-dual" in the sense that there is a representation $V$ of $G$ and maps in $\mathcal{S} \mathcal{W}^{G}$

$$
\begin{aligned}
K \wedge L & \rightarrow S^{V} \\
S^{V} & \rightarrow L \wedge K
\end{aligned}
$$

with the property that the composites

$$
\begin{gathered}
S^{V} \wedge L \rightarrow L \wedge K \wedge L \rightarrow L \wedge S^{V} \\
K \wedge S^{V} \rightarrow K \wedge L \wedge S^{V} \rightarrow S^{V} \wedge K
\end{gathered}
$$

are the symmetry isomorphism. Then for $X, Y \in \pi^{\text {st }} \mathcal{S}^{G}$ the composite

$$
\begin{aligned}
\pi^{\mathrm{st}} \mathcal{S}^{G}(X, Y \wedge K) \rightarrow & \pi^{\mathrm{st}} \mathcal{S}^{G}(X \wedge L, Y \wedge K \wedge L) \\
& \rightarrow \pi^{\mathrm{st}} \mathcal{S}^{G}\left(X \wedge L, Y \wedge S^{V}\right) \approx \pi^{\mathrm{st}} \mathcal{S}^{G}\left(S^{-V} \wedge X \wedge L, Y\right)
\end{aligned}
$$

is an isomorphism, by the standard duality manipulation.

Given $X \rightarrow Y \in \mathcal{S}^{G}$, and any $Z$ there is a long exact sequence

$$
\begin{array}{r}
\cdots \rightarrow \pi^{\mathrm{st}} \mathcal{S}^{G}\left(Z, S^{k} \wedge X\right) \rightarrow \pi^{\mathrm{st}} \mathcal{S}^{G}\left(Z, S^{k} \wedge Y\right) \rightarrow \pi^{\mathrm{st}} \mathcal{S}^{G}\left(Z, S^{k} \wedge(Y \cup C X)\right) \\
\rightarrow \pi^{\mathrm{st}} \mathcal{S}^{G}\left(Z, S^{k+1} \wedge X\right) \rightarrow \cdots .
\end{array}
$$

As in the proof of B.20, this is proved with the argument of [48, III.2.1], using the analogue of Proposition B.19 given as the special case of Corollary B.34 in which $V$ is trivial. 
There is also an easier long exact sequence in the other variable. Let $A \rightarrow X$ be a map in $\mathcal{S}^{G}$ and $Y$ any spectrum. Then there is a long exact sequence (B.38)

$$
\cdots \rightarrow \pi^{\mathrm{st}} \mathcal{S}^{G}\left(S^{k} \wedge(X \cup C A), Y\right) \rightarrow \pi^{\mathrm{st}} \mathcal{S}^{G}\left(S^{k} \wedge X, Y\right) \rightarrow \pi^{\mathrm{st}} \mathcal{S}^{G}\left(S^{k} \wedge A, Y\right) \rightarrow \cdots
$$

Under the isomorphism given by Proposition B.28, this is the long exact sequence of Proposition B.18 associated to the fibration sequence of function spectra

$$
Y^{X \cup C A} \rightarrow Y^{X} \rightarrow Y^{A}
$$

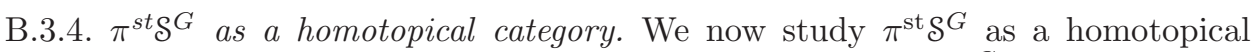
category, and in doing so establish the fact that the functor $\mathcal{S} \mathcal{W}^{G} \rightarrow$ ho $\mathcal{S}^{G}$ is fully faithful.

By Proposition B.28 the functors $\pi_{k}^{H}$ factor through $\pi^{\text {st }} \mathcal{g}^{G}$. We make $\pi^{\text {st }} \mathcal{g}^{G}$ into a homotopical category by defining a map to be a weak equivalence if it induces an isomorphism in $\pi_{k}^{H}$ for all $H \subset G$ and all $k \in \mathbb{Z}$. Since a map in $\mathcal{S}^{G}$ is a weak equivalence if and only if it is so in $\pi^{\text {st }} \mathcal{S}^{G}$, the canonical functor

$$
\text { ho } \mathcal{S}^{G} \rightarrow \text { ho } \pi^{\text {st }} \mathcal{S}^{G}
$$

is an isomorphism. Corollary B.6 asserts that if $X \in \mathcal{S}^{G}$ happens to have the property that $\pi^{\text {st } \mathcal{S}^{G}}(X,-)$ is a homotopy functor, then $\pi^{\text {st }} \mathcal{S}^{G}(X,-) \rightarrow$ ho $\pi^{\text {st }} \mathcal{S}^{G}(X,-)$ is an isomorphism. Combining this with the isomorphism (B.39) gives

Lemma B.40. If $X \in \mathcal{S}^{G}$ has the property that $\pi^{\text {st }} \mathcal{S}^{G}(X,-)$ is a homotopy functor, then for all $Y$, the maps

$$
\pi^{s t} \mathcal{S}^{G}(X, Y) \rightarrow \operatorname{ho} \pi^{s t} \mathcal{S}^{G}(X, Y) \stackrel{\sim}{\leftarrow} \operatorname{ho}^{G}(X, Y)
$$

are isomorphisms, and so ho $\mathcal{S}^{G}(X, Y)$ may be computed as $\pi^{\text {st }} \mathcal{S}^{G}(X, Y)$.

Proposition B.42. For $k \in \mathbb{Z}$ the maps (B.29) and (B.41) give isomorphisms

$$
\pi_{k}^{H} X \approx \pi^{s t} \mathcal{S}^{G}\left(G / H_{+} \wedge S^{k}, X\right) \approx \operatorname{ho}^{G}\left(G / H_{+} \wedge S^{k}, X\right) .
$$

Proof: The first isomorphism is given by Proposition B.28, and it implies that $\pi^{\text {st }} \mathcal{S}^{G}\left(G / H_{+} \wedge S^{k}, X\right)$ is a homotopy functor of $X$. Lemma B.40 then gives the second isomorphism.

Corollary B.43. A map $X \rightarrow Y$ in $\mathcal{S}^{G}$ is a weak equivalence if and only if it becomes an isomorphism in ho $\mathcal{S}^{G}$.

Proposition B.44. When $X$ is of the form $X=S^{\ell} \wedge K$ with $K$ a finite $G$-CW complex, and $\ell \in \mathbb{Z}$, the functor $\pi^{\text {st }} \mathcal{S}^{G}(X,-)$ is a homotopy functor, and so for all $Y$ ho $\mathcal{S}^{G}(X, Y)$ may be computed as $\pi^{\text {st }} \mathcal{S}^{G}(X, Y)$.

Proof: Working through the skeletal filtration of $K$ and using the exact sequence (B.38) reduces the claim to the case in which $K=G / H_{+} \wedge S^{n}$. But that case is Corollary B.28.

Note that

$$
\pi^{\mathrm{st}} \mathcal{S}^{G}\left(S^{0} \wedge K, S^{0} \wedge L\right)=\underset{\lim _{\longrightarrow}}{ } \pi_{0} \mathcal{T}^{G}\left(S^{V} \wedge K, S^{V} \wedge L\right) .
$$

When $L$ is a finite $G$-CW complex, this is the definition of $\delta W^{G}(K, L)$. Thus Proposition B.44 contains as a special case 
Proposition B.45. The functor $\Sigma^{\infty}$ induces a fully faithful embedding $\mathcal{S W}^{G} \rightarrow$ ho $\mathcal{S}^{G}$.

B.3.5. Equivariant additivity. Our next goal is to show that the formation of indexed wedges in $\mathcal{S}^{G}$ is homotopical. We will do this, as in [5], via a SpanierWhitehead duality argument. To make this work we need to show that smashing with $S^{V}$ and $S^{-V}$ are homotopical. As mentioned in Remark B.35, this implies that they induce inverse functors on ho $\mathcal{S}^{G}$. It also lays the groundwork for our investigation of the homotopical properties of the smash product in $\S$ B.3.7.

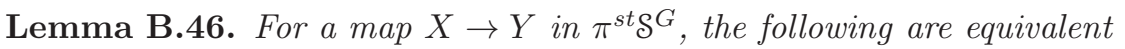

i) The $\operatorname{map} X \rightarrow Y$ is a weak equivalence.

ii) For all $H \subset G$ and all $k \in \mathbb{Z}$ the map

$$
\pi^{s t}\left(G / H_{+} \wedge S^{k}, X\right) \rightarrow \pi^{s t}\left(G / H_{+} \wedge S^{k}, Y\right)
$$

is an isomorphism.

iii) For some representation $V$ of $G$, all $H \subset G$ and all $k \in \mathbb{Z}$ the map

$$
\pi^{s t}\left(G / H_{+} \wedge S^{k} \wedge S^{V}, X\right) \rightarrow \pi^{s t}\left(G / H_{+} \wedge S^{k} \wedge S^{V}, Y\right)
$$

is an isomorphism.

iv) For all representations $V$ of $G$, all $H \subset G$ and all $k \in \mathbb{Z}$ the map

$$
\pi^{s t}\left(G / H_{+} \wedge S^{k} \wedge S^{V}, X\right) \rightarrow \pi^{s t}\left(G / H_{+} \wedge S^{k} \wedge S^{V}, Y\right)
$$

is an isomorphism.

Proof: The equivalence of the first two statements in Proposition B.42, and they imply the fourth by Proposition B.44. The fourth statement obviously implies the third. That the third statement implies the first two is proved by induction on $|G|$, the assertion being trivial when $G$ is trivial. We may therefore assume that part iii) holds, and that part ii) holds for all proper $H \subset G$. Let $V_{0} \subset V$ be the subspace of invariant vectors. Using the long exact sequence (B.38), and working by downward induction through an equivariant cell decomposition of $S^{V}$, one sees that for all $k \in \mathbb{Z}$ and all $H \subset G$, our assumptions imply that the map

$$
\pi^{\mathrm{st}}\left(G / H_{+} \wedge S^{k} \wedge S^{V_{0}}, X\right) \rightarrow \pi^{\mathrm{st}}\left(G / H_{+} \wedge S^{k} \wedge S^{V_{0}}, Y\right)
$$

is an isomorphism. But in $\pi^{\mathrm{st}} \mathcal{g}^{G}$ there is an isomorphism $S^{k} \wedge S^{V_{0}} \approx S^{k+\ell}$ with $\ell=\operatorname{dim} V_{0}$, so this implies part ii).

We next show that both smashing with $S^{V}$ and smashing with $S^{-V}$ are homotopical functors. Combined with Corollary B.34 this implies that they induce inverse equivalences of ho $\mathcal{S}^{G}$.

Proposition B.47. Let $V$ be a representation of $G$. The following conditions on a map $X \rightarrow Y \in \pi^{\text {st }} \mathcal{S}^{G}$ are equivalent

i) The map $X \rightarrow Y$ is a weak equivalence

ii) The map $S^{V} \wedge X \rightarrow S^{V} \wedge Y$ is a weak equivalence

iii) The map $S^{-V} \wedge X \rightarrow S^{-V} \wedge Y$ is a weak equivalence. 
Proof: Since smashing with $S^{V}$ is the inverse equivalence of smashing with $S^{-V}$ it suffices to establish the equivalence of the first two assertions. Now for any $X$, smashing with $S^{V}$ gives an isomorphism

$$
\pi^{\text {st }}\left(G / H_{+} \wedge S^{k}, S^{-V} \wedge X\right) \approx \pi^{\text {st }}\left(G / H_{+} \wedge S^{k} \wedge S^{V}, X\right),
$$

so the equivalence of the first two assertions is a consequence of Lemma B.46.

Corollary B.48. Suppose that $V$ is a representation of $G$. Smashing with $S^{V}$ and $S^{-V}$ are inverse equivalences of ho $\mathcal{S}^{G}$.

With Proposition B.47 in place, we have the following generalization of Proposition B.44.

Proposition B.49. When $X$ is of the form $X=S^{-V} \wedge K$, with $K$ a finite $G-C W$ complex, the functor $\pi^{\text {st } \mathcal{S}^{G}}(X,-)$ is a homotopy functor, hence

$$
\pi^{s t} \mathcal{S}^{G}(X,-) \rightarrow \operatorname{ho}^{G}(X,-)
$$

is an isomorphism.

Proof: By Corollary B.34 there is an isomorphism.

$$
\pi^{\text {st }} \mathcal{S}^{G}\left(S^{-V} \wedge K,(-)\right) \approx \pi^{\text {st }} \mathcal{S}^{G}\left(K, S^{V} \wedge(-)\right) .
$$

But $S^{V} \wedge(-)$ is a homotopy functor by Proposition B.47, and $\pi^{\text {st } \mathcal{S}^{G}}(K,(-))$ is a homotopy functor by Proposition B.44.

Expanded out, Proposition B.49 gives the formula

$$
\operatorname{ho} \mathcal{S}^{G}\left(S^{-V} \wedge K, Y\right)=\underset{W}{\lim }\left[S^{W} \wedge K, Y_{V \oplus W}\right]^{G}
$$

advertised in $\S 2.2 .4$ as (2.19). Taking $S^{-V} \wedge K$ to be $S^{-V} \wedge S^{k} \wedge G / H_{+}, k \in \mathbb{Z}$, this specializes to the isomorphism

$$
\operatorname{hos}^{G}\left(S^{-V} \wedge S^{k} \wedge G / H_{+}, X\right) \approx \lim _{W>-k} \pi_{W+k}^{H} X_{V \oplus W} .
$$

In particular, the expression $\lim _{W>-k} \pi_{W+k}^{H} X_{V \oplus W}$ is a homotopy functor of $X$. This fact is used in the proof of Proposition B.69, which plays a fundamental role in establishing the positive complete stable model category structure on $\mathcal{S}^{G}$.

The fact that $\pi^{\text {st }} \mathcal{S}^{G}$ is tensored over $\mathcal{S} \mathcal{W}^{G}$ also gives control over homotopical properties of the smash product and of indexed wedges.

Corollary B.51. If $X$ is of the form $S^{-W} \wedge K$, with $K$ a $G$-CW complex and $W$ a representation of $G$, then the functor

$$
(-) \wedge X: \mathcal{S}^{G} \rightarrow \mathcal{S}^{G}
$$

is homotopical.

Proof: By Proposition B.17 we may assume $K$ to be finite. In addition, it suffices to show that smashing with $S^{-W} \wedge K$ is homotopical as a functor from $\pi^{\text {st }} \mathcal{S}^{G}$ to itself. Suppose that $Y \rightarrow Y^{\prime}$ is a weak equivalence. Let $L \in \mathcal{S} \mathcal{W}^{G}$ be a $V$-dual of $K$. By the isomorphism of Proposition B.28 it suffices to show that for all $H \subset G$ and all $k \in \mathbb{Z}$, the map

$$
\pi^{\mathrm{st}} \mathcal{S}^{G}\left(G / H_{+} \wedge S^{k}, Y \wedge X\right) \rightarrow \pi^{\mathrm{st}} \mathcal{S}^{G}\left(G / H_{+} \wedge S^{k}, Y^{\prime} \wedge X\right)
$$


is an isomorphism. Using the first part of the duality isomorphism (B.36), we can identify this map with

$$
\pi^{\mathrm{st}} \mathcal{S}^{G}\left(G / H_{+} \wedge S^{k} \wedge S^{W} \wedge L, S^{V} \wedge Y\right) \rightarrow \pi^{\mathrm{st}} \mathcal{S}^{G}\left(G / H_{+} \wedge S^{k} \wedge S^{W} \wedge L, S^{V} \wedge Y^{\prime}\right),
$$

and finally by Proposition B.44, with

$$
\operatorname{ho} \mathcal{S}^{G}\left(G / H_{+} \wedge S^{k} \wedge S^{W} \wedge L, S^{V} \wedge Y\right) \rightarrow \operatorname{ho} \mathcal{S}^{G}\left(G / H_{+} \wedge S^{k} \wedge S^{W} \wedge L, S^{V} \wedge Y^{\prime}\right) \text {. }
$$

But this latter map is an isomorphism since $S^{V} \wedge Y \rightarrow S^{V} \wedge Y^{\prime}$ is a weak equivalence (Proposition B.47).

Proposition B.52. Let $J$ be a finite $G$-set. For any $X \in \mathcal{S}^{G}$, the canonical map

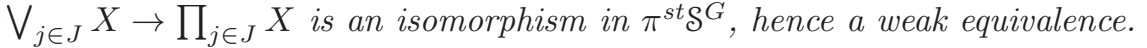

Proof: The finite $G$-sets are self-dual in $\mathcal{S} \mathcal{W}^{G}$. Since

$$
\bigvee_{j \in J} X \approx J_{+} \wedge X
$$

the result follows from the duality isomorphism

$$
\pi^{\mathrm{st}} \mathcal{G}^{G}\left(Z, J_{+} \wedge X\right) \approx \pi^{\mathrm{st}} \mathcal{S}^{G}\left(J_{+} \wedge Z, X\right) \approx \pi^{\mathrm{st}} \mathcal{S}^{G}\left(Z, \prod_{j \in J} X\right)
$$

once one checks that the composite map is the same as the one coming from the canonical map from the (constant) finite indexed wedge to the finite indexed product. We leave this to the reader.

Corollary B.53. Let $J$ be a finite $G$-set and $X$ an equivariant $J$-diagram. The map

$$
\bigvee_{j \in J} X_{j} \rightarrow \prod_{j \in J} X_{j}
$$

is an isomorphism in $\pi^{s t} \mathcal{S}^{G}$, hence a weak equivalence.

Proof: Let $U: \mathcal{S}^{G} \rightarrow \mathcal{S}^{B_{J} G}$ be the pullback map associated to the unique equivariant map $J \rightarrow$ pt. The indexed wedge is the left adjoint to $U$ and the indexed product is the right adjoint. The natural transformation from the indexed wedge to the indexed product is easily checked to satisfy the condition of Lemma B.54 below. This reduces us to checking the case in which the $J$-diagram is constant at a $G$-spectrum $X$. But that case is covered by Proposition B.52.

We have used

Lemma B.54. Suppose that $U: \mathcal{D} \rightarrow \mathcal{C}$ is a functor with a left adjoint $L$ and right adjoint $R$, and that $L \rightarrow R$ is a natural transformation. If the composition

$$
\text { Id } \rightarrow U R \rightarrow \text { Id }
$$

of the adjoint to $L \rightarrow R$ with the counit of the adjunction is the identity, then $L \rightarrow R$ is a retract of $L U R \rightarrow R U R$. 
Proof: Just apply $L \rightarrow R$ on the left to the composition (B.55) to get

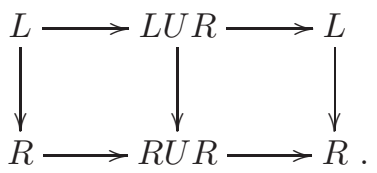

Corollary B.53 implies the only non-trivial part of the following "indexed" analogue of Proposition B.22.

Proposition B.56. i) The formation of finite indexed products is homotopical.

ii) Suppose that $J$ is a finite $G$-set, and $X: \mathcal{B}_{J} G \rightarrow \mathcal{S}$ is a functor. The map

$$
\bigvee_{j \in J} X_{j} \rightarrow \prod_{j \in J} X_{j}
$$

is a stable weak equivalence in $\mathcal{S}^{G}$. Hence the formation of finite indexed wedges is homotopical.

iii) The formation of all indexed wedges is homotopical.

B.3.6. Change of group. Let $H \subset G$ be a subgroup. Specializing Proposition B.56 to the case $J=G / H$ gives the homotopical properties of the "change of group" functors. The functor $i_{H}^{*}: \mathcal{S}^{G} \rightarrow \mathcal{S}^{H}$ is homotopical by definition, and so induces a functor on the homotopy categories

$$
i_{H}^{*}: \operatorname{ho} \mathcal{S}^{G} \rightarrow \operatorname{ho} \mathcal{S}^{H} .
$$

Taking $J=G / H$ in Proposition B.56 we see that the left and right adjoints to $i_{H}^{*}$ are also homotopical, and that the canonical natural transformation between them is a weak equivalence. They therefore induce left and right adjoints to the restriction map on the homotopy categories, and the canonical map between them is an isomorphism. This is the Wirthmüller isomorphism [84, 5].

B.3.7. Weak equivalences and the smash product. The smash product is not known to preserve weak equivalences, but it does so in good cases.

Definition B.57. An equivariant orthogonal spectrum is cellular if it is in the smallest subcategory of $\mathcal{S}^{G}$ containing the spectra of the form $G_{+}{ }_{H} S^{-V} \wedge S^{k}$ with $V$ a representation of $H$ and $k \geq 0$ and which is closed under the formation of arbitrary coproducts, the formation of mapping cones, and the formation of filtered colimits along $h$-cofibrations.

The small object argument shows that every $X$ receives, functorially, a weak equivalence $\tilde{X} \rightarrow X$ from a cellular $\tilde{X}$.

Proposition B.58. If $K$ is cellular then $K$ is flat: the functor $X \mapsto X \wedge K$ preserves weak equivalences.

Proof: By Corollary B.51 and the fact that the formation of indexed wedges is homotopical (Proposition B.56) the result is true when $K=G_{+}{ }_{H}^{\wedge} S^{-V} \wedge S^{k}$. The functor $X \wedge K$ is built from

$$
X \wedge G_{+}{ }_{H} S^{-V} \wedge S^{k}
$$


by forming wedges, mapping cones, and filtered colimits along $h$-cofibrations, all of which are homotopical by Proposition B.56.

Since every object is weakly equivalent to a cellular object, and cellular objects are flat, Remark B.16 implies

Proposition B.59. Suppose that $X \rightarrow Y$ is a weak equivalence of flat spectra. Then for any $Z$, the map $X \wedge Z \rightarrow Y \wedge Z$ is a weak equivalence.

Let $\mathcal{S}_{\mathrm{fl}}^{G} \subset \mathcal{S}^{G}$ be the full subcategory of flat objects, considered as a homotopical category using the stable weak equivalences. Since every object of $\mathcal{S}^{G}$ is weakly equivalent to an object of $\mathcal{S}_{\mathrm{fl}}^{G}$, the functor

$$
\text { ho } \mathcal{S}_{\mathrm{fl}}^{G} \rightarrow \text { ho } \mathcal{S}^{G}
$$

is an equivalence of categories. The above results show

Proposition B.61. The smash product functor

$$
\mathcal{S}_{f l}^{G} \times \mathcal{S}^{G} \rightarrow \mathcal{S}^{G}
$$

is homotopical.

The equivalence (B.60) and Proposition B.58 are enough to show that the smash product descends to give ho $\mathcal{S}^{G}$ a symmetric monoidal structure, and that the map $\mathcal{S} \mathcal{W}^{G} \rightarrow$ ho $^{G}$ is symmetric monoidal. For a more refined statement, see $\S B$.4.2.

\section{B.4. Spectra as a model category.}

B.4.1. The positive complete model structure. Let $\mathcal{A}_{\text {cof }}$ be the set of maps

$$
\mathcal{A}_{\text {cof }}=\left\{G_{+}{ }_{H}^{\wedge} S^{-V} \wedge S_{+}^{n-1} \rightarrow G_{+}{ }_{H}^{\wedge} S^{-V} \wedge D_{+}^{n}\right\}
$$

with $n \geq 0, H$ a subgroup of $G$ and $V$ a representation of $H$ containing a non-zero invariant vector. We define the class

$$
\mathcal{S}_{\text {cof }}^{G} \subset \mathcal{S}^{G}
$$

of positive complete cofibrations to be the smallest collection of maps in $\mathcal{S}^{G}$ containing the maps in (B.62) and which is closed under coproducts, cobase change along arbitrary maps, and filtered colimits. A positive complete fibration (or just fibration) is a map having the right lifting property with respect to the class of maps in $\mathcal{S}_{\text {cof }}^{G}$ which are stable weak equivalences.

Proposition B.63. The category $\mathcal{S}^{G}$ equipped with the stable weak equivalences, the positive complete cofibrations and the positive complete fibrations forms a (cofibrantly generated) Quillen model category.

We will call this model structure the positive complete model structure, and when we need to recruit a model structure for some task, this will be the one we use. Henceforth the terms "cofibration," "fibration" and "weak equivalence" will refer to "positive complete cofibration," "positive complete fibration," and "stable weak equivalence."

Remark B.64. Since the maps in $\mathcal{A}_{\text {cof }}$ are mapping cylinders they are $h$-cofibrations. This implies that the cofibrations in $\mathcal{S}^{G}$ are $h$-cofibrations (cf. [53, Lemma III.2.5]) and hence flat. The cofibrant objects in $\mathcal{S}^{G}$ are cellular hence flat. 
The "positive" condition is needed for the study of commutative algebras. On the other hand, it creates some peculiarities in the model structure. For example, the zero sphere $S^{0}$ is not cofibrant, nor is $S^{0} \wedge K$ when $K$ is a $G$-CW complex. The cofibrant replacements are given by

$$
S^{-1} \wedge S^{1} \wedge K \rightarrow S^{0} \wedge K
$$

This means that the adjunction

$$
\Sigma^{\infty}: \mathcal{T}^{G} \leftrightarrows \mathcal{S}^{G}: \Omega^{\infty}
$$

is not a Quillen adjunction, even though the left adjoint preserves all weak equivalences between non-degenerately based $G$-spaces, and so barely needs to be derived.

The positive complete model structure does not quite appear in the literature. It is closely related to the positive stable model structure of [53].

The positive complete model structure is cofibrantly generated. The set $\mathcal{A}_{\text {cof }}$ is the set of generating cofibrations. The set $\mathcal{B}_{\text {acyclic }}$ of generating acyclic cofibrations consists of the analogous maps

$$
G_{+}{ }_{H}^{\wedge} S^{-V} \wedge I_{+}^{n-1} \rightarrow G_{+}{ }_{H}^{\wedge} S^{-V} \wedge I^{n}
$$

together with the corner maps formed by smashing

$$
G_{+} \underset{H}{\wedge}\left(S^{-V \oplus W} \wedge S^{W}\right) \rightarrow G_{+} \underset{H}{\wedge} \tilde{S}^{-V}
$$

with the maps $S_{+}^{n-1} \rightarrow D_{+}^{n}$. The $H$-representation $V$ is assumed to have a nonzero invariant vector, while $W$ need not. The map (B.66) is extracted from the factorization

$$
S^{-V \oplus W} \wedge S^{W} \rightarrow \tilde{S}^{-V} \rightarrow S^{-V}
$$

formed by applying the small object construction in $\mathcal{S}^{H}$, using the maps in $\mathcal{A}_{\text {cof }}$.

A map $X \rightarrow Y$ has the right lifting property with respect to the class of maps $\mathcal{A}_{\text {cof }}$ if and only for each $H \subset G$ and each representation $V$ of $H$ containing a non-zero invariant vector, the map $X_{V} \rightarrow Y_{V}$ is an acyclic fibration in $\mathcal{T}^{H}$. Among other things this implies that $X \rightarrow Y$ is a weak equivalence and that the map $\tilde{S}^{-V} \rightarrow S^{-V}$ is a homotopy equivalence. From this one concludes that a map $X \rightarrow Y$ has the right lifting property with respect to $\mathcal{B}_{\text {acyclic }}$ if and only if for each subgroup $H \subset G$ and each representation $V$ of $H$ containing a non-zero invariant vector, the map $X_{V} \rightarrow Y_{V}$ is a fibration in $\mathcal{S}^{H}$, and for each representation $W$ of $H$ the square

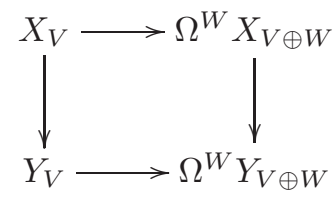

is homotopy cartesian in $\mathcal{T}^{H}$.

Proposition B.69. If a map $X \rightarrow Y$ is a weak equivalence and has the right lifting property with respect to $\mathcal{B}_{\text {acyclic }}$ then it has the right lifting property with respect to $\mathcal{A}_{\text {cof }}$. 
Proof: We must show that the conditions imply that for each $H \subset G$ and each representation $V$ of $H$ containing a non-zero invariant vector, the map $X_{V} \rightarrow Y_{V}$ is an acyclic fibration in $\mathcal{T}^{H}$. Part of our assumption is that it is a fibration, so it remains to show that it is a weak equivalence. Choose an exhausting sequence $\left\{V_{n}\right\}$. Letting $W$ range through this sequence in (B.68) leads to a homotopy cartesian square

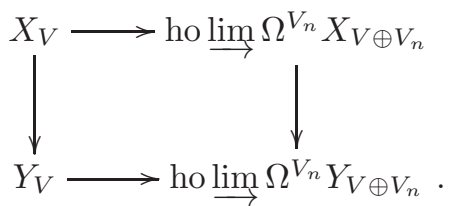

Since $X \rightarrow Y$ is a weak equivalence, the rightmost vertical map is a weak equivalence (by (B.50)), hence so is $X_{V} \rightarrow Y_{V}$.

Proposition B.70. Any cobase change along a map in $\mathcal{B}_{\text {acyclic }}$ is a weak equivalence.

Proof: Since the maps in $\mathcal{B}_{\text {acyclic }}$ are flat, it suffices to check that the maps in $\mathcal{B}_{\text {acyclic }}$ are weak equivalences. The only ones for which this is not obvious are the corner maps. Since they are flat, it suffices to check that the quotients

$$
G_{+}{ }_{H}\left(\tilde{S}^{-W} /\left(S^{-V \oplus W} \wedge S^{V}\right)\right) \wedge D^{m} / S^{m}
$$

are weakly contractible. Since $D^{m} / S^{m}$ is flat, and $G_{+} \underset{H}{\wedge}(-)$ is homotopical, it suffices to show that

$$
\tilde{S}^{-W} /\left(S^{-V \oplus W} \wedge S^{V}\right)
$$

is weakly contractible in $\mathcal{S}^{H}$, or, equivalently that the leftmost map in (B.67) is a weak equivalence in $\mathcal{S}^{H}$. But that is a consequence of Proposition B.30 and the two out of three property.

Proposition B.71. A map $X \rightarrow Y$ is a fibration if and only if it has the right lifting property with respect to $\mathcal{B}_{\text {acyclic }}$

Proof: Suppose that $A \rightarrow B$ is an acyclic cofibration. Using the small object

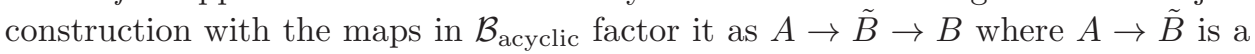
filtered colimit of maps constructed by iterated cobase change along maps in $\mathcal{B}_{\text {acyclic }}$ and $\tilde{B} \rightarrow B$ has the right lifting property with respect to $\mathcal{B}_{\text {acyclic. The map } A \rightarrow \tilde{B}}$ is a weak equivalence by Propositions B.70 and B.17. It follows that $\tilde{B} \rightarrow B$ is a weak equivalence, and so by Proposition B.69, has the right lifting property with respect to $\mathcal{A}_{\text {cof }}$. This means that $A \rightarrow B$ is a retract of $A \rightarrow \tilde{B}$. Since $X \rightarrow Y$ has the right lifting property for $A \rightarrow \tilde{B}$ it also has this property for $A \rightarrow B$.

The verification of the model category axioms is now completely straightforward and left to the reader.

Let $H \subset G$ be a subgroup. In the positive complete model category structures, the restriction functor

$$
i_{H}^{*}: \mathcal{S}^{G} \rightarrow \mathcal{S}^{H}
$$

preserves weak equivalences, fibrations and cofibrations. This implies 
Proposition B.72. Let $H \subset G$ be a subgroup. The restriction functor and its left adjoint form a Quillen pair

$$
G_{+}{ }_{H}(-): \mathcal{S}^{H} \leftrightarrows \mathcal{S}^{G}: i_{H}^{*},
$$

as do the restriction functor and its right adjoint

$$
i_{H}^{*}: \mathcal{S}^{G} \leftrightarrows \mathcal{S}^{H}: \prod_{j \in G / H}(-)_{j}
$$

Corollary B.73. An indexed wedge of cofibrations is a cofibration.

Corollary B.73 is one of our reasons for introducing the positive complete model structure. The positive stable model structure of [53] does not have this property.

Associated to any map $i: G^{\prime} \rightarrow G$ of finite groups is a functor $i^{*}: \mathcal{S}^{G} \rightarrow \mathcal{S}^{G^{\prime}}$. This functor has both a left and right adjoint. The functor $i^{*}$ sends the generating cofibrations to indexed wedges of generating cofibrations, hence cofibrations by Corollary B.73. Since it is a left adjoint it therefore sends cofibrations to cofibrations. It also sends the generating acyclic cofibrations to weak equivalences. To see this note that the generators of the form $X \wedge\left(I_{+}^{n-1} \rightarrow I_{+}^{n}\right)$ are homotopy equivalences hence go to homotopy equivalences. To check that the corner maps go to weak equivalences, it suffices to show that the maps (B.66) go to weak equivalences. Since $\tilde{S}^{-V} \rightarrow S^{-V}$ is a homotopy equivalence, this is equivalent to showing that maps of the form

$$
G_{+}{ }_{H}\left(S^{-V \oplus W} \wedge S^{W}\right) \rightarrow G_{+}{ }_{H}^{\wedge} S^{-V}
$$

go to weak equivalences. But these maps go to an indexed wedge of maps of the form

$$
\left(S^{-V^{\prime} \oplus W^{\prime}} \wedge S^{W^{\prime}}\right) \rightarrow S^{-V^{\prime}}
$$

which are weak equivalences. Thus $i^{*}$ also sends acyclic cofibrations to acyclic cofibrations. This gives

Proposition B.75. If $i: G^{\prime} \rightarrow G$ is any homomorphism of finite groups, then the pullback functor

$$
i^{*}: \mathcal{S}^{G} \rightarrow \mathcal{S}^{G^{\prime}}
$$

is a left Quillen functor.

For more along these lines see [53, Remark V.3.13]

B.4.2. Smash product. Equipped with the smash product and the positive complete model category structure, $\mathcal{S}^{G}$ is a symmetric monoidal model category in the sense of Hovey [34, Definition 4.2.6] and Schwede-Shipley [75]. This means that the analogue of Quillen's axiom SM7 holds (the pushout product axiom), and for any cofibrant $X$, the map

$$
\tilde{S}^{0} \wedge X \rightarrow X
$$

is a weak equivalence, where $\tilde{S}^{0} \rightarrow S^{0}$ is a cofibrant approximation. As will be apparent to the reader the proof applies equally well if "cofibration" is replaced by "cellular."

Proposition B.76. Equipped with the smash product, the positive complete model structure is a symmetric monoidal model category. 
The positive complete model structure also satisfies the monoid axiom [76, Definition 3.3].

Proposition B.77. If $X \rightarrow Y$ is an acyclic cofibration in $\mathcal{S}^{G}$, and $Z$ is arbitrary then $X \wedge Z \rightarrow Y \wedge Z$ is a flat weak equivalence.

We have stated these together to slightly streamline the proof. When cofibrations are flat, the monoid axiom implies the "weak equivalence" part of the pushout product axiom once one knows the "cofibration" part. Indeed suppose $A_{1} \rightarrow B_{1}$ is an acyclic cofibration and $A_{2} \rightarrow B_{2}$ is a cofibration. Then the vertical arrows in the diagram

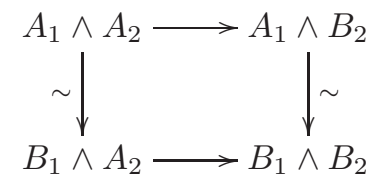

are weak equivalences by the monoid axiom, and all of the arrows are cofibrations by the "cofibration" part (Remark B.79). Since cofibrations are flat, the map from $A_{1} \wedge B_{2}$ to the pushout is a weak equivalence, and the desired weak equivalence assertion then follows from two out of three.

Proofs of Propositions B.76 and B.7\%: The unit axiom follows from Proposition B.59 since cofibrant objects are cellular, hence flat (Remark B.64). The pushout product axiom asserts that if $f_{1}: A_{1} \rightarrow B_{1}$ and $f_{2}: A_{2} \rightarrow B_{2}$ are cofibrations, then the corner map from the pushout of the left and top arrows in

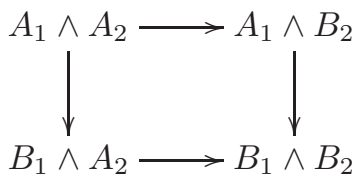

to the bottom right term is a cofibration, and is acyclic if one of $f_{1}$ or $f_{2}$ is. It suffices to check the cofibration condition when $f_{1}$, and $f_{2}$ are in $\mathcal{A}_{\text {cof }}$ and so of the form

$$
\begin{aligned}
& G \underset{H_{1}}{\wedge} S^{-V_{1}} \wedge\left(S^{k-1} \rightarrow D^{k}\right) \\
& G \underset{H_{2}}{\wedge} S^{-V_{2}} \wedge\left(S^{\ell-1} \rightarrow D^{\ell}\right) .
\end{aligned}
$$

But in that case the corner map is the smash product of $G \underset{H_{1}}{\wedge} S^{-V_{1}}$ with $G \underset{H_{2}}{\wedge} S^{-V_{2}}$ with the pushout product of $S^{k-1} \rightarrow D^{k}$ and $S^{\ell-1} \rightarrow D^{\ell}$. This is an indexed wedge of cofibrations hence a cofibration. As remarked above, once Proposition B.77 is proved, we are done. Since $X \rightarrow Y$ is a cofibration it is an $h$-cofibration, so it suffices to show that $(Y / X) \wedge Z$ is weakly contractible if $Y / X$ is. But $Y / X$ is cofibrant, hence flat, so the claim follows from Proposition B.59.

Remark B.79. The special case of the pushout product axiom for $* \rightarrow A$ and $* \rightarrow B$ asserts that if $A$ and $B$ are cofibrant, then so is $A \wedge B$.

Hovey [34, Theorem 4.3.2] now implies

Corollary B.80. The left derived smash product makes ho $\mathcal{S}^{G}$ into a complete symmetric monoidal category. 
B.4.3. The canonical homotopy presentation. Let

$$
\cdots \subset V_{n} \subset V_{n+1} \subset \ldots
$$

be an exhausting sequence of orthogonal $G$-representations, and consider the transition diagram

$$
S^{-V_{n+1} \wedge} \underset{S^{-V_{n}} \wedge X_{n} .}{ }\left(V_{n}, V_{n+1}\right) \wedge X_{n} \longrightarrow S^{-V_{n+1}} \wedge X_{n+1}
$$

Write

$$
W_{n}=V_{n+1}-V_{n}
$$

for the orthogonal complement of $V_{n}$ in $V_{n+1}$. The inclusion $V_{n} \subset V_{n+1}$ gives an embedding

$$
S^{W_{n}} \rightarrow \mathscr{J}_{G}\left(V_{n}, V_{n+1}\right),
$$

and so from (B.81) a diagram

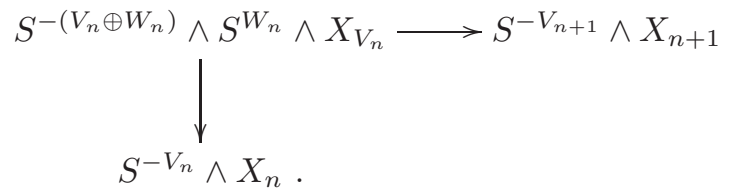

Putting these together as $n$ varies results in a system

$$
A_{0} \stackrel{\sim}{\leftarrow} B_{0} \rightarrow A_{1} \stackrel{\sim}{\leftarrow} B_{1} \rightarrow A_{2} \stackrel{\sim}{\leftarrow} B_{2} \rightarrow A_{3} \stackrel{\sim}{\leftarrow} B_{3} \rightarrow \ldots .
$$

The system (B.82) maps to $X$ and a simple check of equivariant stable homotopy groups shows that the map from its homotopy colimit to $X$ is a weak equivalence. Now for each $n$ let $C_{n}$ be the homotopy colimit of the portion

$$
A_{0} \stackrel{\simeq}{\leftarrow} B_{0} \rightarrow \ldots \rightarrow A_{n-1} \stackrel{\sim}{\leftarrow} B_{n-1} \rightarrow A_{n}
$$

of (B.82). Then $C_{n}$ is naturally weakly equivalent to $A_{n}=S^{-V_{n}} \wedge X_{V_{n}}$, and the $C_{n}$ fit into a sequence

$$
C_{0} \rightarrow C_{1} \rightarrow C_{2} \rightarrow \ldots
$$

whose homotopy colimit coincides with that of (B.82). This gives the canonical homotopy presentation of $X$. One can functorially replace the sequence (B.84) with a weakly equivalent sequence of cofibrations between cofibrant-fibrant objects. The colimit of this sequence is naturally weakly equivalent to $X$. It will be cofibrant automatically, and fibrant since the model category $\mathcal{S}^{G}$ is compactly generated.

We write the canonical homotopy presentation of $X$ as

$$
X \approx \underset{V_{n}}{\operatorname{holim}}\left(S^{-V_{n}} \wedge X_{V_{n}}\right)_{\mathrm{cf}},
$$

or when more precision is needed, as a diagram

$$
X \leftarrow \underset{V_{n}}{\operatorname{holim}}\left(S^{-V_{n}} \wedge X_{V_{n}}\right)_{\mathrm{c}} \rightarrow \underset{V_{n}}{\operatorname{holim}}\left(S^{-V_{n}} \wedge X_{V_{n}}\right)_{\mathrm{cf}},
$$

with the subscript indicating cofibrant and cofibrant-fibrant replacement. 
B.5. Homotopy properties of the norm. The purpose of this section is to establish Proposition B.105 which asserts that indexed smash products have a left derived functor which may be computed on cofibrant objects. As will be apparent to the reader, they can also be computed on cellular objects. Many of the technical results in this section are also required for our analysis of symmetric powers and of commutative algebras.

Before formulating our main results, we generalize the situation slightly.

B.5.1. Equivariant $J$-diagrams. Given a non-empty $G$-set $J$, consider the category $\mathcal{S}^{\mathcal{B}_{J} G}$ of functors $\mathcal{B}_{J} G \rightarrow \mathcal{S}$. A choice of point $t$ in each $G$-orbit of $J$ gives an equivalence

$$
\mathcal{S}^{\mathcal{B}_{J} G} \approx \prod_{t} \mathcal{S}^{G_{t}}
$$

where $G_{t}$ is the stabilizer of $t$. We give $\mathcal{S}^{\mathcal{B}_{J} G}$ the model structure corresponding to the product of the positive complete model structures under this equivalence. The model structure is independent of the chosen points in each orbit. We will refer to the model category $\mathcal{S}^{\mathcal{B}_{J} G}$ as the model category of equivariant $J$-diagrams of spectra.

To be more explicit, a map of $J$-diagrams $X \rightarrow Y$ is a weak equivalence if and only for each $j \in J$ the map $X j \rightarrow Y j$ is a weak equivalence in $\mathcal{S}^{G_{j}}$. The generating cofibrations are the maps whose $j^{\text {th }}$ component has the form

$$
G_{j} \underset{H_{j}}{\wedge} S^{-V_{j}} \wedge S_{+}^{m_{j}-1} \rightarrow G_{j}+{ }_{H_{j}}^{\wedge} S^{-V_{j}} \wedge D_{+}^{m_{j}}
$$

with $V_{j}$ a representation of $H_{j}$ having a non-zero invariant vector. They can be expressed without reference to points and stabilizers as an indexed wedge

$$
p_{*}^{\vee}\left(S^{-V} \wedge\left(S_{+}^{n-1} \rightarrow D_{+}^{n}\right)\right)
$$

with $p: J^{\prime} \rightarrow J$ a finite surjective map of $G$-sets, and $V$ a $G$-equivariant orthogonal vector bundle over $J^{\prime}$ having a nowhere-zero invariant section. The generating acyclic cofibrations are the maps of the form

$$
p_{*}^{\vee} S^{-V} \wedge\left(I_{+}^{n-1} \rightarrow I_{+}^{n}\right)
$$

and those constructed as the corner map formed by smashing

$$
p_{*}^{\vee}\left(S^{-V \oplus W} \wedge S^{W} \rightarrow \tilde{S}^{-V}\right)
$$

with the maps $S_{+}^{n-1} \rightarrow D_{+}^{n}$. As in (B.66), the map (B.86) is extracted from the factorization

$$
S^{-V \oplus W} \wedge S^{W} \rightarrow \tilde{S}^{-V} \rightarrow S^{-V}
$$

by applying the small object construction in the category of equivariant $J^{\prime}$-diagrams using the generating cofibrations. The map $\tilde{S}^{-V} \rightarrow S^{V}$ is a homotopy equivalence.

If $J \rightarrow K$ is a map of finite $G$-sets, the restriction functor

$$
\mathcal{S}^{B_{K} G} \rightarrow \mathcal{S}^{B_{J} G}
$$

has both a left and right adjoint, given by the two Kan extensions. All three functors are homotopical, and the both the restriction functor and its left adjoint send cofibrations to cofibrations. This means that the restriction functor is both a left and right Quillen functor. 
Let $p: J \rightarrow K$ be an equivariant map of finite $G$-sets. The indexed smash product gives a functor

$$
p_{*}^{\wedge}=(-)^{\wedge J / K}: \mathcal{S}^{\mathcal{B}_{J} G} \rightarrow \mathcal{S}^{\mathcal{B}_{K} G} .
$$

When $J \rightarrow K$ is the map $G / H \rightarrow$ pt this is the norm. The various homotopical properties of indexed and symmetric smash products we require are most naturally expressed as properties of $(-)^{\wedge J / K}$. Working fiberwise, establishing these reduces to the case $K=$ pt. To keep the discussion uncluttered we focus on that case in this section, leaving the extension to the case of more general $K$ to the reader.

B.5.2. Indexed smash products and cofibrations. Let $p: J \rightarrow$ pt be the unique equivariant map and write the indexed smash product as $(-)^{\wedge J}$. Note that if $V$ is an equivariant orthogonal vector bundle over $J$ then

$$
\left(S^{-V}\right)^{\wedge J}=S^{-V^{\prime}},
$$

where $V^{\prime}$ is the orthogonal $G$-space of global sections of $V$.

Lemma B.88. Suppose that $A \rightarrow B$ is a generating cofibration in $\mathcal{S}^{\mathcal{B}_{J} G}$. The indexed corner map $\partial_{A} B^{\wedge J} \rightarrow B^{\wedge J}$ is an indexed wedge

$$
\bigvee_{\Gamma} S^{-V} \wedge\left(S(W)_{+} \rightarrow D(W)_{+}\right)
$$

in which $\Gamma$ is a $G$-set, $V$ and $W$ are equivariant vector bundles over $\Gamma$ and $V$ has a non-zero invariant section. In particular, $\partial_{A} B^{\wedge J} \rightarrow B^{\wedge J}$ is a cofibration.

Proof: This is a straightforward consequence of the distributive law (Theorem A.37) applied to (B.85), and the compatibility of the formation of $\partial_{A} B^{\wedge J}$ with indexed wedges, as described at the end of $\S$ A.3.4.

Proposition B.89. Suppose that $J$ is a non-empty finite $G$-set. If $X \rightarrow Y$ is a cofibration of equivariant $J$-diagrams, the indexed smash product

$$
X^{\wedge J} \rightarrow Y^{\wedge J}
$$

is an h-cofibration. It is a cofibration between cofibrant objects in $\mathcal{S}^{G}$ if $X$ is cofibrant.

Proof: The assertion that $X^{\wedge J} \rightarrow Y^{\wedge J}$ is an $h$-cofibration is contained in Proposition A.69. For the cofibration assertion we work by induction on $|J|$, and may therefore assume the result to be known for any non-empty $J_{0} \subset J$ and any $H \subset G$ stabilizing $J_{0}$ as a subset. In particular, we may assume that if $X$ is cofibrant, then $X^{\wedge J_{0}}$ is a cofibrant $H$-spectrum for any non-empty proper $J_{0} \subset J$ and any $H \subset G$ stabilizing $J_{0}$ as a subset.

We will establish the theorem in the case in which $X \rightarrow Y$ arises from a pushout square of $J$-diagrams

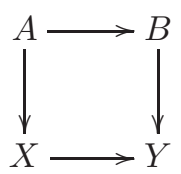

in which $A \rightarrow B$ is a generating cofibration. We will show in this case that $X^{\wedge J} \rightarrow Y^{\wedge J}$ is an $h$-cofibration, and is a cofibration if $X$ is cofibrant. Since the 
formation of indexed smash products commutes with directed colimits and retracts, the proposition then follows from the small object argument.

Give $Y^{\wedge J}$ the filtration described in $\S A .3 .4$. The successive terms are related by the pushout square

$$
\begin{gathered}
\bigvee_{\substack{J=J_{0} \amalg J_{1} \\
\left|J_{1}\right|=n}} X^{\wedge J_{0}} \wedge \partial_{A} B^{\wedge J_{1}} \longrightarrow \bigvee_{\substack{J=J_{0} \amalg J_{1} \\
\left|J_{1}\right|=n}} X^{\wedge J_{0}} \wedge B^{\wedge J_{1}} \\
\downarrow \\
\operatorname{fil}_{n-1} Y^{\wedge J} \longrightarrow \operatorname{fil}_{n} Y^{\wedge J} .
\end{gathered}
$$

By Lemma B.88, each of the maps

$$
\partial_{A} B^{\wedge J_{1}} \rightarrow B^{\wedge J_{1}}
$$

is a cofibration. If $X$ is cofibrant, then $X^{\wedge J_{0}}$ is either $S^{0}$ or cofibrant by induction, hence

$$
X^{\wedge J_{0}} \wedge \partial_{A} B^{\wedge J_{1}} \rightarrow X^{\wedge J_{0}} \wedge B^{\wedge J_{1}}
$$

is a cofibration by the pushout product axiom. Since indexed wedges preserve cofibrations, the top row of (B.90) is then a cofibration and hence so is the bottom row.

To show that the indexed smash product has a left derived functor we need to augment Proposition B.89 and show that what when $X \rightarrow Y$ is an acyclic cofibration, then $X^{\wedge J} \rightarrow Y^{\wedge J}$ is a weak equivalence. This can be proved with the above argument once we know that the indexed corner maps $\partial_{A} B^{\wedge J} \rightarrow B^{\wedge J}$ associated to the generating acyclic cofibrations are weak equivalences. But the generating acyclic cofibrations contain the maps of the form (B.86) so dealing with them requires understanding something about indexed corner maps of fairly general cofibrations. These can be studied as the indexed smash products of maps in a different symmetric monoidal category.

B.5.3. The category of arrows. Let $\mathcal{S}_{1}^{G}$ denote the category of maps $X=\left(X_{0} \rightarrow X_{1}\right)$ in $\mathcal{S}^{G}$, with morphisms the commutative diagrams. As mentioned in Remark A.42, $\mathcal{S}_{1}^{G}$ can be made into a closed symmetric monoidal category by defining

$$
\left(X_{1} \rightarrow X_{2}\right) \wedge\left(Y_{1} \rightarrow Y_{2}\right)
$$

to be the corner map, from the pushout of the top and left arrows in

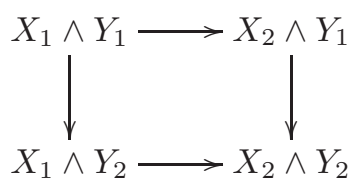

to the bottom right corner. The tensor unit is $* \rightarrow S^{0}$.

We give $\mathcal{S}_{1}^{G}$ the projective model structure in which a map

$$
\left(X_{1} \rightarrow X_{2}\right) \rightarrow\left(Y_{1} \rightarrow Y_{2}\right)
$$

is a weak equivalence or fibration if each of $X_{i} \rightarrow Y_{i}$ is, and is a cofibration if both $X_{1} \rightarrow Y_{1}$ and the corner map

$$
X_{2} \underset{X_{1}}{\cup} Y_{1} \rightarrow Y_{2}
$$


are cofibrations. An object $X_{1} \rightarrow X_{2}$ is therefore cofibrant if $X_{1}$ is cofibrant and $X_{1} \rightarrow X_{2}$ is a cofibration.

The model structure on $\mathcal{S}_{1}^{G}$ is compactly generated. The generating (acyclic) cofibrations in $\mathcal{S}_{1}^{G}$ are of two types. Type I are the maps

$$
(K \rightarrow K) \rightarrow(L \rightarrow L)
$$

and type II are the maps

$$
(* \rightarrow K) \rightarrow(* \rightarrow L)
$$

were $K \rightarrow L$ is running through the set $\mathcal{A}_{\text {cof }}$ defined in (B.62) (respectively $\mathcal{B}_{\text {acyclic }}$ ).

Proposition B.93. Equipped with the structure just described, $\mathcal{S}_{1}^{G}$ is a symmetric monoidal model category satisfying the monoid axiom.

Proof: The proof follows the proof of Propositions B.76 and B.77, and, because of the special nature of the generators, essentially reduces to it. It suffices to check the "cofibration" assertion on generators. In each of the three cases (type I and type I, type II and type II, and mixed type) the result reduces to the case of $\mathcal{S}^{G}$. Since the cofibrations are $h$-cofibration, the monoid axiom reduces showing that if $(* \rightarrow *) \rightarrow\left(X_{1} \rightarrow X_{2}\right)$ is an acyclic cofibration and $\left(Z_{1} \rightarrow Z_{2}\right)$ is arbitrary, then both the domain and range in the corner map of

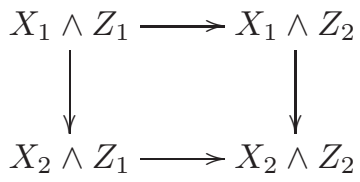

are weakly contractible. But by the monoid axiom for $\mathcal{S}^{G}$, every term in the diagram is weakly contractible. The claim then follows since the left vertical arrow is an $h$-cofibration, hence flat. As pointed out before the statement of Proposition B.76, this implies the "weak equivalence" part of the pushout product axiom. The unit axiom is also straightforward and left to the reader.

The proof of Proposition B.93 is more or less completely formal, and can be rewritten to apply to the arrow category of any symmetric monoidal model category. This is done in the recent paper [33] of Hovey.

B.5.4. Indexed corner maps and cofibrations. Proposition B.93 addresses the homotopy properties of ordinary smash products in $\mathcal{S}_{1}^{G}$. For the indexed smash products we work in the arrow category $\mathcal{S}_{1}^{\mathcal{B}_{J} G}$ of maps of equivariant $J$-diagrams, in the projective model structure. Our aim is to establish Proposition B.96 which gives control over the indexed corner maps in $\mathcal{S}^{G}$ (Proposition B.97). It is the analogue in $\mathcal{S}_{1}^{\mathcal{B}}{ }_{J} G$ of Proposition B.89. In preparation, we need to identify the generating (acyclic) cofibrations. As mentioned in the previous section, those in $\mathcal{S}_{1}^{G}$ are of two types. Type I are the maps

$$
(K \rightarrow K) \rightarrow(L \rightarrow L)
$$

and type II are the maps

$$
(* \rightarrow K) \rightarrow(* \rightarrow L)
$$

were $K \rightarrow L$ is running through the set $\mathcal{A}_{\text {cof }}$ defined in (B.62) (respectively $\mathcal{B}_{\text {acyclic }}$ ). The generating (acyclic) cofibrations in $\mathcal{S}_{1}^{\mathcal{B}_{J} G}$ can be taken to be the equivariant $J$-diagrams consisting entirely of type I or type II generators. 
Remark B.94. A map (B.91) is an $h$-cofibration if both $X_{1} \rightarrow Y_{1}$ and the corner map (B.92) are. Since the cofibrations in $\mathcal{S}^{G}$ are $h$-cofibrations the same is true of the cofibrations in $\mathcal{S}_{1}^{G}$.

Lemma B.95. If $X \rightarrow Y$ is a generating cofibration in the category of equivariant $J$-diagrams in $\mathcal{S}_{1}^{G}$, then the indexed corner map

$$
\partial_{X} Y^{\wedge J} \rightarrow Y^{\wedge J}
$$

is a cofibration between cofibrant objects in $\mathcal{S}_{1}^{G}$.

Proof: First note that for generating cofibrations of type I, the corner map is

$$
\left(\partial_{K} L^{\wedge J} \rightarrow \partial_{K} L^{\wedge J}\right) \rightarrow\left(L^{\wedge J} \rightarrow L^{\wedge J}\right)
$$

and in type II it is

$$
\left(* \rightarrow \partial_{K} L^{\wedge J}\right) \rightarrow\left(* \rightarrow L^{\wedge J}\right) .
$$

The assertion therefore reduces to Lemma B.88.

Proposition B.96. Suppose that $J$ is a finite $G$-set. If

$$
X \rightarrow Y
$$

is a cofibration in $\mathcal{S}_{1}^{G}$ and $X$ is cofibrant, then the indexed smash product

$$
X^{\wedge J} \rightarrow Y^{\wedge J}
$$

is a cofibration between cofibrant objects.

Proof: The proof proceeds exactly as in the case of Proposition B.89. The filtration of $\S$ A.3.4 and induction on $|J|$ reduce the problem to showing that the indexed corner map (in $\mathcal{S}_{1}^{\mathcal{B}_{J} G}$ )

$$
\partial_{A} Y^{\wedge J} \rightarrow B^{\wedge J}
$$

is a cofibration between cofibrant objects, when $A \rightarrow B$ is a cofibrant generator. This is the content of Lemma B.95.

Specializing, we now have

Proposition B.97. If $X \rightarrow Y$ is a cofibration of equivariant J-diagrams and $X$ is cofibrant, then the indexed corner map $\partial_{X} Y^{\wedge J} \rightarrow Y^{\wedge J}$ is a cofibration between cofibrant objects.

Proof: If $X \rightarrow Y$ is a cofibration of cofibrant $J$-diagrams, then $(X \rightarrow Y)$ is cofibrant $J$-diagram in $\mathcal{S}_{1}^{G}$, and so

$$
(X \rightarrow Y)^{\wedge J}=\left(\partial_{X} Y^{\wedge J} \rightarrow Y^{\wedge J}\right)
$$

is cofibrant by Proposition B.96.

The result below is not used elsewhere in this paper, but is useful in other contexts. Having come this far, we record it here.

Proposition B.98. Suppose that $X \rightarrow Y \rightarrow Z$ is a sequence of cofibrations of equivariant $J$-diagrams in $\mathcal{S}^{G}$, and that $X$ is cofibrant. Then the map

$$
\partial_{X} Z^{\wedge J} \rightarrow \partial_{Y} Z^{\wedge J}
$$

is a cofibration between cofibrant objects. 
Proof: Define $Y \rightarrow Z^{\prime}$ by the pushout square

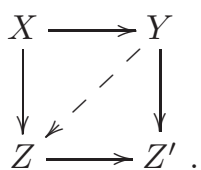

As the dashed arrow indicates, the map $(X \rightarrow Z) \rightarrow(Y \rightarrow Z)$ is a retract of $(X \rightarrow Z) \rightarrow\left(Y \rightarrow Z^{\prime}\right)$. By Proposition B.96 the map

$$
\left(\partial_{X} Z^{\wedge J} \rightarrow Z^{\wedge J}\right) \rightarrow\left(\partial_{Y} Z^{\wedge J} \rightarrow Z^{\wedge J}\right)
$$

is a cofibration, hence so is the map

$$
\partial_{X} Z^{\wedge J} \rightarrow \partial_{Y} Z^{\wedge J}
$$

and therefore so is its retract

$$
\partial_{X} Z^{\wedge J} \rightarrow \partial_{Y} Z^{\wedge J}
$$

B.5.5. Indexed smash products and acyclic cofibrations. With the indexed corner maps of cofibrations under control we can now turn to the acyclic cofibrations.

Lemma B.99. If $X \rightarrow Y$ is a generating acyclic cofibration in $\mathcal{S}^{\mathcal{B}_{J} G}$, then the indexed corner map

$$
\partial_{X} Y^{\wedge J} \rightarrow Y^{\wedge J}
$$

is an acyclic cofibration of cofibrant objects in $\mathcal{S}^{G}$.

Proof: We know from Proposition B.97 that the indexed corner maps are cofibrations between cofibrant objects, so what remains is the assertion that they are weak equivalences. This can be reduced further. Suppose that $X \rightarrow Y$ is an acyclic cofibration in $\mathcal{S}^{\mathcal{B}_{J} G}$ and we wish to show that the indexed corner map $\partial_{X} Y^{\wedge J} \rightarrow Y^{\wedge J}$ is a weak equivalence. Give $Y^{\wedge J}$ the filtration described in $\S A .3 .4$, in which the successive terms are related by the pushout square

$$
\begin{gathered}
\bigvee_{\substack{J=J_{0} \amalg J_{1} \\
\left|J_{1}\right|=n}} X^{\wedge J_{0}} \wedge \partial_{X} Y^{\wedge J_{1}} \longrightarrow \bigvee_{\substack{J=J_{0} \amalg J_{1} \\
\left|J_{1}\right|=n}} X^{\wedge J_{0}} \wedge Y^{\wedge J_{1}} \\
\operatorname{fil}_{n-1} Y^{\wedge J} \longrightarrow \text { fil }_{n} Y^{\wedge J} .
\end{gathered}
$$

By Proposition B.97 and the pushout product axiom, the upper arrow is a cofibration, which, by induction on $|J|$, we may assume to be acyclic when $n<|J|$. Since the cofibrations are flat, this means that the bottom arrow is an acyclic cofibration when $n<|J|$. It follows that in this case, the indexed corner map is a weak equivalence if and only if the absolute map $X^{\wedge J} \rightarrow Y^{\wedge J}$ is.

We now turn to the generating acyclic cofibrations. The generators of the form $X \wedge\left(I_{+}^{n-1} \rightarrow I_{+}^{n}\right)$ are homotopy equivalences, hence so are the absolute maps. The other generators are of the form

$$
\left(S_{+}^{n-1} \rightarrow D^{n_{+}}\right) \wedge\left(p_{*}^{\vee} S^{-V \oplus W} \wedge S^{W} \rightarrow p_{*}^{\vee} \tilde{S}^{-V}\right),
$$

where $p: J^{\prime} \rightarrow J$ is a map of finite $G$-sets and $V$ and $W$ are equivariant vector bundles over $J^{\prime}$. The fact that the norm is multiplicative, together with the monoid 
axiom for $\mathcal{S}_{1}^{G}$, reduces us to considering only the right hand factor in (B.100). The distributive law further reduces us to the case $J^{\prime}=J$. Finally, since the map $\tilde{S}^{-V} \rightarrow S^{V}$ is a homotopy equivalence, we may replace $\tilde{S}^{V}$ with $S^{V}$. Evaluating both sides using Proposition A.59 we see that the assertion amounts to checking that

$$
S^{-V^{\prime} \oplus W^{\prime}} \wedge S^{W^{\prime}} \rightarrow S^{-V^{\prime}}
$$

is a weak equivalence, where $V^{\prime}$ and $W^{\prime}$ are the $G$-spaces of global sections. But this is Proposition B.30 (see Remark B.33).

As with Lemma B.95, the separate cases of type I and type II generators reduce the result below to Lemma B.99.

Lemma B.101. If $X \rightarrow Y$ is a generating acyclic cofibration in the category of equivariant $J$-diagrams in $\mathcal{S}_{1}^{G}$, then the indexed corner map

$$
\partial_{X} Y^{\wedge J} \rightarrow Y^{\wedge J}
$$

is an acyclic cofibration of cofibrant objects in $\mathcal{S}_{1}^{G}$.

Proposition B.102. Suppose that $J$ is a finite $G$-set. The functor

$$
(-)^{\wedge J}: \mathcal{S}_{1}^{\mathcal{B}_{J} G} \rightarrow \mathcal{S}_{1}^{G}
$$

sends acyclic cofibrations between cofibrant objects to acyclic cofibration between cofibrant objects, and hence weak equivalences between cofibrant objects to weak equivalences between cofibrant objects.

Proof: The proof proceeds exactly as in the case of Proposition B.89. That the second assertion follows from the first is Ken Brown's Lemma (see, for example [34, Lemma 1.1.12]).

Specializing Proposition B.102, we have

Proposition B.103. If $X \rightarrow Y$ is an acyclic cofibration in $\mathcal{S}^{\mathcal{B}_{J} G}$ and $X$ is cofibrant, then both the indexed corner map $\partial_{X} Y^{\wedge J} \rightarrow Y^{\wedge J}$ and the absolute map $X^{\wedge J} \rightarrow Y^{\wedge J}$ are acyclic cofibrations between cofibrant objects.

B.5.6. Homotopy properties of the norm. With all this in hand we can now show that indexed smash products have left derived functors. From Proposition B.89, Proposition B.103, and Ken Brown's Lemma we have

Proposition B.104. The indexed smash product

$$
(-)^{\wedge J}: \mathcal{S}^{\mathcal{B}_{J} G} \rightarrow \mathcal{S}^{G}
$$

takes weak equivalences between cofibrant objects to weak equivalences between cofibrant objects.

This gives

Proposition B.105. The indexed smash product has a left derived functor

$$
(-)^{\wedge}{ }^{\mathrm{L} J}: \mathcal{S}^{\mathcal{B}_{J} G} \rightarrow \operatorname{ho} \mathcal{S}^{G}
$$

which may be computed as

$$
X^{\stackrel{\mathrm{L}}{\wedge}}=\left(X_{c}\right)^{\wedge J}
$$

where $X_{c} \rightarrow X$ is a cofibrant approximation. 
B.6. Symmetric powers. We now turn to the homotopical properties of symmetric smash powers, or just "symmetric powers" for short.

B.6.1. Indexed symmetric powers. The $n^{\text {th }}$ symmetric (smash) power of a $G$-spectrum is the orbit spectrum

$$
\operatorname{Sym}^{n}(X)=X^{\wedge n} / \Sigma_{n} .
$$

The homotopy properties of this functor are fundamental to understanding the homotopy theory of equivariant commutative algebras. For indexed smash products of commutative algebras the distributive law leads one to consider indexed smash products of symmetric powers

$$
\left(\operatorname{Sym}^{n} X\right)^{\wedge J} .
$$

These can be written as

$$
\left(\operatorname{Sym}^{n} X\right)^{\wedge J}=\left(X^{\wedge n} / \Sigma_{n}\right)^{\wedge J} \approx X^{\wedge(\mathbf{n} \times J)} / \Sigma_{n}^{J}
$$

with $\mathbf{n}=\{1, \ldots, n\}$. This last expression is an indexed symmetric power. The definition and homotopy properties of indexed symmetric powers are the subject of this section.

Before turning to the definition, we consider a more basic situation. Suppose that $i: \tilde{G} \rightarrow G$ is a surjective map of groups with kernel $N$. Then the functor $i^{*}: \mathcal{S}^{G} \rightarrow \mathcal{S}^{\tilde{G}}$ has both a left and a right adjoint. This is most readily understood by thinking of $G$-spectra as objects of $\mathcal{S}$ equipped with a $G$-action. The left adjoint $i_{!}: \mathcal{S}^{\tilde{G}} \rightarrow \mathcal{S}^{G}$ sends a spectrum $Y$ to the orbit spectrum $Y / N$ equipped with its residual $G$-action. The expression on the right of (B.106) is a special case of this. As in any diagram category, the orbit spectrum $Y / N$ is computed objectwise: if $U$ is an orthogonal vector space then $(Y / N)_{U}$ is the $G$-space $Y_{U} / N$. For the homotopical properties we need information about the $W$-space, for a representation $W$ of $G$. It is given by the formula

$$
(Y / N)_{W}=O(U, W)_{+} \underset{O(U)}{\wedge}(Y / N)_{U}
$$

where $U$ is any vector space of the same dimension as $W$ but with trivial $G$-action. Interchanging the colimits, this space can be written as

$$
\left(O(U, W)+\underset{O(U)}{\wedge} Y_{U}\right) / N
$$

which, in turn is isomorphic to

$$
Y_{W} / N
$$

where now $W$ is regarded as a $\tilde{G}$ representation through the map $\tilde{G} \rightarrow G$.

We can now define indexed symmetric powers. Let $I$ be a finite $G$-set, and $\Sigma_{I}$ the group of (not necessarily equivariant) automorphisms of $I$, with $G$ acting by conjugation. Fix a $G$-stable subgroup $\Sigma \subset \Sigma_{I}$ and regard $I$ as a $\Sigma \rtimes G$-set through the projection map to $G$. For a $\Sigma \rtimes G$-equivariant $I$-diagram $X$ the indexed symmetric power is the orbit $G$-spectrum

$$
\operatorname{Sym}_{\Sigma}^{I} X=X^{\wedge I} / \Sigma .
$$

When the indexing set $I$ has a trivial $G$-action, $\Sigma$ is the full symmetry group of $I$, and the equivariant $I$-diagram is the constant diagram with value $X \in \mathcal{S}^{G}$, then this construction is the usual symmetric power $\operatorname{Sym}^{|I|} X$ discussed above. We will usually not distinguish in notation between a $\Sigma \rtimes G$-spectrum $X$ and the constant equivariant $I$-diagram with value $X$. 
If $X \rightarrow Y$ is a map of $\Sigma \rtimes G$-equivariant $I$-diagrams, the indexed symmetric corner map is the map of orbit $G$-spectra

$$
\partial_{X} \operatorname{Sym}_{\Sigma}^{I} Y \rightarrow \operatorname{Sym}_{\Sigma}^{I} Y
$$

obtained by passing to $\Sigma$ orbits from

$$
\partial_{X} Y^{\wedge I} \rightarrow Y^{\wedge I} .
$$

It can also be regarded as the symmetric power $\operatorname{Sym}_{\Sigma}^{I}(X \rightarrow Y)$ of $(X \rightarrow Y)$ regarded as an object of the arrow category $\mathcal{S}_{1}^{\mathcal{B}_{\Sigma \rtimes G} I}$.

Remark B.107. Since the orbit spectrum functor is a continuous left adjoint, it sends $h$-cofibrations to $h$-cofibrations. For example, suppose that $X \rightarrow Y$ is a cofibration of cofibrant $\Sigma \rtimes G$-equivariant $I$-diagrams. By Proposition B.89 and Proposition B.97 both the indexed smash product

$$
X^{\wedge I} \rightarrow Y^{\wedge I}
$$

and the corner map

$$
\partial_{X} Y^{\wedge I} \rightarrow Y^{\wedge I}
$$

are cofibrations, and hence $h$-cofibrations, of $\Sigma \rtimes G$-spectra. This means that all four of the maps

$$
\begin{aligned}
\operatorname{Sym}_{\Sigma}^{I} X & \rightarrow \operatorname{Sym}_{\Sigma}^{I} Y \\
\partial_{X} \operatorname{Sym}_{\Sigma}^{I} Y & \rightarrow \operatorname{Sym}_{\Sigma}^{I} Y \\
\left(E_{G} \Sigma\right)_{+} \hat{\Sigma}^{\wedge} X^{\wedge I} & \rightarrow\left(E_{G} \Sigma\right)_{+}{ }_{\Sigma} Y^{\wedge I} \\
\left(E_{G} \Sigma\right)_{+}{ }_{\Sigma} \partial_{X} Y^{\wedge I} & \rightarrow\left(E_{G} \Sigma\right)_{+}{ }_{\Sigma} Y^{\wedge I}
\end{aligned}
$$

are $h$-cofibrations of $G$-spectra.

Note that $X^{\wedge I}$ with its $\Sigma \rtimes G$-action is a special case of an indexed monoidal product. This means that the distributive law applies to symmetric powers, and, given a pushout square

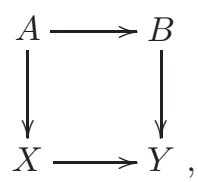

there is a filtration of $\operatorname{Sym}_{\Sigma}^{I} Y$ whose successive terms are related by passing to $\Sigma$-orbits from the filtration described in $\S$ A.3.4.

As described in [53], the homotopy theoretic analysis of indexed symmetric powers requires certain equivariant principal bundles. For the moment, let $\Sigma$ be any finite group with a $G$-action.

Definition B.108. An equivariant universal $\Sigma$-space is a $\Sigma \rtimes G$-space $E_{G} \Sigma$ with the property that for each finite $\Sigma \rtimes G$-set $S$, the space of $\Sigma \rtimes G$-equivariant maps

$$
S \rightarrow E_{G} \Sigma
$$

is empty if some element of $S$ is fixed by a non-identity element of $\Sigma$, and contractible otherwise. 
The defining property characterizes an equivariant universal $\Sigma$-space up to $\Sigma \rtimes G$ equivariant weak homotopy equivalence. The space $E_{G} \Sigma$ is the total space of the universal $G$-equivariant principal $\Sigma$-bundle. It can be constructed as a $\Sigma \rtimes G$-CW complex, with cells of the form $S \times D^{m}$, where $S$ is a $\Sigma$-free $\Sigma \rtimes G$-set. We will always assume our equivariant universal $\Sigma$-spaces are $\Sigma \rtimes G$-CW complexes, in which case the characterization is up to equivariant homotopy equivalence.

The symmetric powers of a cofibrant spectrum are rarely cofibrant. However they still have very good homotopy theoretic properties. Our main result is the following.

Proposition B.109. Suppose that $X \rightarrow Y$ is a cofibration between cofibrant $\Sigma \rtimes G$ equivariant I-diagrams. In the square of $G$-spectra

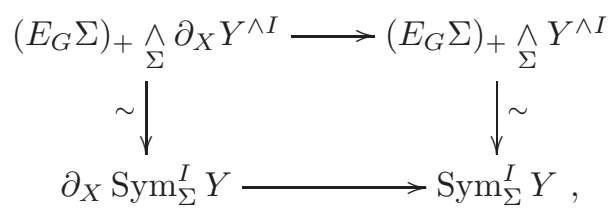

every object is flat, the upper row is a cofibration between cofibrant objects, the vertical maps are weak equivalences, and the bottom row is an h-cofibration. The horizontal maps are weak equivalences if $X \rightarrow Y$ is.

Remark B.111. By Proposition B.59 the maps in (B.110) asserted to be weak equivalences remain so after smashing with any spectrum $Z$.

Remark B.112. The situation that comes up in studying the free commutative algebra functor is that $X \rightarrow Y$ is a cofibration of cofibrant $G$-spectra, regarded as a $\Sigma_{I} \rtimes G$-spectrum through the map to $G$, and then regarded as a constant equivariant $I$-diagram. This map of equivariant $I$-diagrams is cofibrant by Proposition B.75, and so Proposition B.109 applies.

Along the way to proving Proposition B.109 we will also show

Proposition B.113. The functors $\left(E_{G} \Sigma\right)_{+} \hat{\Sigma}_{\Sigma}(-)^{\wedge I}$ and $\operatorname{Sym}_{\Sigma}^{I}(-)$ take weak equivalences between cofibrant objects to weak equivalences.

Remark B.114. Proposition B.109 is part the reason for the positive condition in the model structure we have chosen. The result is not true for general cellular objects described in $\S \mathrm{B} .3 .7$, though it is true for cellular object built from cells of the form $G_{+} \bigwedge_{H} S^{-V} \wedge D_{+}^{k}$ with $V$ non-zero. The condition that $V$ is non-zero is used in the proof of Proposition B.117.

We assertions about the top row in Proposition B.109 are most easily analyzed in the arrow category $\mathcal{S}_{1}^{\mathcal{B}_{I} G}$.

Lemma B.115. The functor

$$
E_{G} \Sigma_{+} \hat{\Sigma}(-)^{\wedge I}: \mathcal{S}_{1}^{\mathcal{B}_{\Sigma \rtimes G} I} \rightarrow \mathcal{S}_{1}^{G}
$$

takes acyclic cofibrations between cofibrant objects to acyclic cofibrations between cofibrant objects and hence weak equivalences between cofibrant objects to weak equivalences between cofibrant objects. 
Proof: Let $X \rightarrow Y$ be an acyclic cofibration. By working through an equivariant cell decomposition of $E_{G} \Sigma$ and using SM7 for the topological enrichment we reduce to showing that if $S$ is a $\Sigma$-free $\Sigma \rtimes G$-set, then the map

$$
S_{+} \hat{\Sigma} X^{\wedge I} \rightarrow S_{+} \wedge Y^{\wedge I}
$$

is an acyclic cofibration between cofibrant objects. This is an indexed wedge of maps, indexed by the $\Sigma$-orbits $\mathcal{O} \subset S$. The summand corresponding to $\mathcal{O}$ is the map of $G_{\mathcal{O}}$-spectra

$$
\mathcal{O}_{+} \underset{\Sigma}{\wedge} X^{\wedge I} \rightarrow \mathcal{O}_{+} \wedge Y^{\wedge I}
$$

where $G_{\mathcal{O}} \subset G$ is the subgroup of $G$ preserving $\mathcal{O}$. Since $\mathcal{O}$ is a $\Sigma$-torsor, this is just the map of indexed smash products

$$
X^{\wedge I^{\prime}} \rightarrow Y^{\wedge I^{\prime}}
$$

with $I^{\prime}=\mathcal{O} \underset{\Sigma}{\times} I$, and is an acyclic cofibration between cofibrant objects by Proposition B.102. The second assertion follows from the first by Ken Brown's Lemma.

The vertical maps in (B.110) require a more detailed analysis.

Definition B.116. Suppose that $\Sigma$ is a group with an action of $G$, and that $X$ is a $\Sigma \rtimes G$-spectrum. We will say that $X$ is $\Sigma$-free as a $G$-spectrum if for each orthogonal $G$-representation $W$ the $\Sigma$-action on $X_{W}$ is free away from the base point.

Proposition B.117. If $X$ is a cofibrant $\Sigma \rtimes G$-equivariant I-diagram, and $Z$ is any $\Sigma \rtimes G$-spectrum then $X^{\wedge I} \wedge Z$ is a $\Sigma$-free $G$-spectrum. The map

$$
\left(E_{G} \Sigma\right)_{+} \wedge{ }_{\Sigma}\left(X^{\wedge I} \wedge Z\right) \rightarrow\left(X^{\wedge I} \wedge Z\right) / \Sigma
$$

is a weak equivalence in $\mathcal{S}^{G}$.

Remark B.119. We will mostly be interested in the case in which the $\Sigma$-action on $Z$ is trivial. In that case the equivalence (B.118) takes the form

$$
\left(\left(E_{G} \Sigma\right)_{+} \hat{\Sigma}_{\Sigma} X^{\wedge I}\right) \wedge Z \stackrel{\sim}{\longrightarrow} \operatorname{Sym}_{\Sigma}^{I}(X) \wedge Z
$$

Remark B.120. The proof of Proposition B.117 is nearly identical to that of [53, Lemma III.8.4]. We go through the details because the statement is slightly more general, and in order to correct a minor error in [53]. The statements of [53, Lemma III.8.4], and the related [53, Lemma IV.4.5] both use $E \Sigma_{i}$, whereas the object that should really be used is $E_{G} \Sigma_{i}$. This makes the proofs of [53, Theorem III.8.1] and [55, Theorem 4] on equivariant commutative rings incomplete. The actual homotopical analysis of commutative rings is more or less equivalent to the homotopical analysis of the norm. So it would seem that any correct treatment needs to be built on the theory of the norm.

Proof of Proposition B.11\%: For the first assertion, it suffices to show that if $A \rightarrow B$ is a generating cofibration,

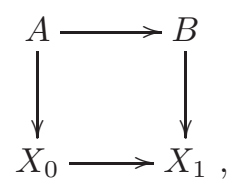


is a pushout square, and $X_{0}^{\wedge I} \wedge Z$ is $\Sigma$-free, then $X_{1}^{\wedge I} \wedge Z$ is $\Sigma$-free. We use the filtration described in $\S$ A.3.4 and consider the pushout square below

$$
\begin{gathered}
\bigvee_{\substack{I=I_{0} \amalg I_{1} \\
\left|I_{1}\right|=m}} X_{0}^{\wedge I_{0}} \wedge \partial_{A} B^{\wedge I_{1}} \wedge Z \longrightarrow \bigvee_{\substack{I=I_{0} \amalg I_{1} \\
\left|I_{1}\right|=m}} X_{0}^{\wedge I_{0}} \wedge B^{\wedge I_{1}} \wedge Z \\
\operatorname{fil}_{m-1} X_{1} \wedge Z \longrightarrow{ }^{\downarrow} \mathrm{fil}_{m} X_{1} \wedge Z .
\end{gathered}
$$

Since $A \rightarrow B$ is a cofibration, the map in the top row is an $h$-cofibration (Proposition B.97) hence a closed inclusion. It therefore suffices to show that $\Sigma$ acts freely away from the base point on the upper right term (see Remark A.9). Induction on $|I|$ reduces this to $m=|I|$. In this way the first assertion of the proposition reduces to checking the special case

$$
X=p_{*}^{\vee} S^{-V} \wedge D_{+}^{k},
$$

with $p: \tilde{I} \rightarrow I$ a surjective map of $\Sigma \rtimes G$-sets and $V$ an equivariant vector bundle over $\tilde{I}$ having a nowhere vanishing invariant global section. Since the factor $\left(D_{+}^{k}\right)^{\wedge I}$ can be absorbed into $Z$, we might as well suppose

$$
X=p_{*}^{\vee} S^{-V} \text {. }
$$

The distributive law gives

$$
X^{\wedge I}=\bigvee_{\gamma \in \Gamma} S^{-V_{\gamma}}
$$

where $\Gamma$ is the $\Sigma \rtimes G$-set of sections $I \rightarrow \tilde{I}$, and

$$
V_{\gamma}=\bigoplus_{i \in I} V_{\gamma(i)} \text {. }
$$

For an orthogonal $\Sigma \rtimes G$-representation $W$ we have, by Lemma A.18,

$$
\left(X^{\wedge I} \wedge Z\right)_{W}= \begin{cases}* & \operatorname{dim} W<\operatorname{dim} V_{\gamma} \\ \bigvee_{\gamma \in \Gamma} O\left(V_{\gamma} \oplus U_{\gamma}, W\right)_{+} \underset{O\left(U_{\gamma}\right)}{\wedge} Z_{U_{\gamma}} & \operatorname{dim} W \geq \operatorname{dim} V_{\gamma}\end{cases}
$$

in which $U=\left\{U_{\gamma}\right\}$ is any $\Sigma \rtimes G$-equivariant vector bundle over $\Gamma$ with $\operatorname{dim} U_{\gamma}=$ $\operatorname{dim} W-\operatorname{dim} V_{\gamma}$. We are interested in representations $W$ which are pulled back from the projection map $\Sigma \rtimes G \rightarrow G$. In the first case there is nothing to prove. In the second case the complement of the base point is homeomorphic to

$$
\coprod_{\gamma \in \Gamma} O\left(V_{\gamma} \oplus U_{\gamma}, W\right) \underset{O\left(U_{\gamma}\right)}{\times}\left(Z_{U_{\gamma}}-\{*\}\right)
$$

(see Remark A.9). The $\Sigma$-freeness then follows from the fact that this space has an equivariant map to the disjoint union of Stiefel-manifolds

$$
\coprod_{\gamma \in \Gamma} O\left(V_{\gamma}, W\right)=\coprod_{\gamma \in \Gamma} O\left(V_{\gamma} \oplus U_{\gamma}, W\right) / O\left(U_{\gamma}\right),
$$

which is $\Sigma$-free since each $V_{\gamma(i)}$ is non-zero, and $\Sigma$ acts faithfully on $I$ but trivially on $W$.

With one additional observation, a similar argument reduces the assertion about weak equivalences to the same case

$$
X=p_{*}^{\vee} S^{-V} .
$$


To spell it out, abbreviate (B.121) as

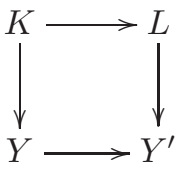

and form

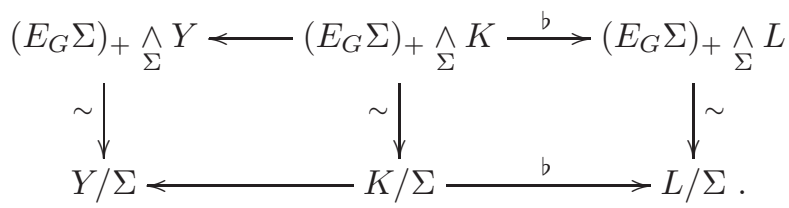

By Remark B.107 the rightmost maps in both rows are $h$-cofibrations, hence flat. This means that if the vertical maps are weak equivalences then the map of pushouts is a weak equivalence (Remark B.13). With this in hand, one now reduces the second claim to the cases $X=p_{*}^{\vee} S^{-V} \wedge S_{+}^{k-1}$ and $X=p_{*}^{\vee} S^{-V} \wedge D_{+}^{k}$. Absorbing the factors $\left(S_{+}^{k-1}\right)^{\wedge I}$ and $\left(D_{+}^{k}\right)^{\wedge I}$ into $Z$ completes the reduction to (B.122).

With this $X$, the map on $W$-spaces induced by (B.118) is the identity map of the terminal object if $\operatorname{dim} W<\operatorname{dim} V_{\gamma}$ and otherwise the map of $\Sigma$-orbit spaces induced by

$$
\left(E_{G} \Sigma\right)_{+} \wedge \bigvee_{\gamma \in \Gamma} O\left(V_{\gamma} \oplus U_{\gamma}, W\right)_{+} \underset{O\left(U_{\gamma}\right)}{\wedge} Z_{U_{\gamma}} \rightarrow \bigvee_{\gamma \in \Gamma} O\left(V_{\gamma} \oplus U_{\gamma}, W\right)_{+} \underset{O\left(U_{\gamma}\right)}{\wedge} Z_{U_{\gamma}}
$$

in which $U=\left\{U_{\gamma}\right\}$ is any $\Sigma \rtimes G$-equivariant vector bundle over $\Gamma$ with $\operatorname{dim} U_{\gamma}=$ $\operatorname{dim} W-\operatorname{dim} V_{\gamma}$. The proposition then follows from the fact that

$$
E_{G} \Sigma \times \coprod_{\gamma \in \Gamma} O\left(V_{\gamma} \oplus U_{\gamma}, W\right) \rightarrow \coprod_{\gamma \in \Gamma} O\left(V_{\gamma} \oplus U_{\gamma}, W\right)
$$

is an equivariant homotopy equivalence for the compact Lie group

$$
\mathscr{G}=\left(\prod_{\gamma \in \Gamma} O\left(U_{\gamma}\right) \rtimes \Sigma\right) \rtimes G .
$$

To see this, note that by $[37,36]$, both sides are $\mathscr{G}$-CW complexes so it suffices to check that the map is a weak equivalence of $H$-fixed point spaces for all $H \subset \mathscr{G}$. If the image of $H$ in $\Sigma \rtimes G$ is not a subgroup of $\Sigma$ then $E_{G} \Sigma^{H}$ is contractible and the map of fixed points is a homotopy equivalence. If $H$ is a subgroup of $\prod O\left(U_{\gamma}\right)$ then it acts trivially on $E_{G} \Sigma$, and once again $E_{G} \Sigma^{H}$ is contractible. Finally, suppose that there is an element $h \in H$ whose image in $\Sigma \rtimes G$ is a non-identity of $\Sigma$. Since $W$ is pulled back from a $G$-representation, this element acts trivially on $W$. If $\gamma \in \Gamma$ is not fixed by $h$ then no point of $O\left(V_{\gamma} \oplus U_{\gamma}, W\right)$ can be fixed by $h$. If $\gamma \in \Gamma$ is fixed by $h$, then $h$ acts on $V_{\gamma}$. This action is non-trivial since $\Sigma$ acts faithfully on $I$. This means that $O\left(V_{\gamma} \oplus U_{\gamma}, W\right)$ has no points fixed by $h$ since $h$ acts trivially on $W$. Both sides therefore have empty $H$-fixed points in this case.

Proof of Proposition B.109: The assertion that the upper arrow is a cofibration between cofibrant objects and a weak equivalence if $X \rightarrow Y$ is, is contained in Lemma B.115. Indeed consider the map of arrows

$$
(X \rightarrow Y) \rightarrow(Y \rightarrow Y) .
$$


If $X \rightarrow Y$ is a cofibration between cofibrant objects then both the domain and range of the above map of arrows are cofibrant. By Lemma B.115 the map

$$
\left(\left(E_{G} \Sigma\right)_{+}{ }_{\Sigma} \partial_{X} Y^{\wedge I} \rightarrow\left(E_{G} \Sigma\right)_{+} \hat{\Sigma}^{\wedge} Y^{\wedge I}\right) \rightarrow\left(\left(E_{G} \Sigma\right)_{+}{ }_{\Sigma} Y^{\wedge I} \rightarrow\left(E_{G} \Sigma\right)_{+} \hat{\Sigma}_{\Sigma} Y^{\wedge I}\right)
$$

is a map of cofibrant objects, which is a weak equivalence if $X \rightarrow Y$ is. This gives the assertion about the upper row. The fact that the bottom row is an $h$-cofibration is part of Remark B.107.

For the remaining assertions it will be helpful to reference the expanded diagram

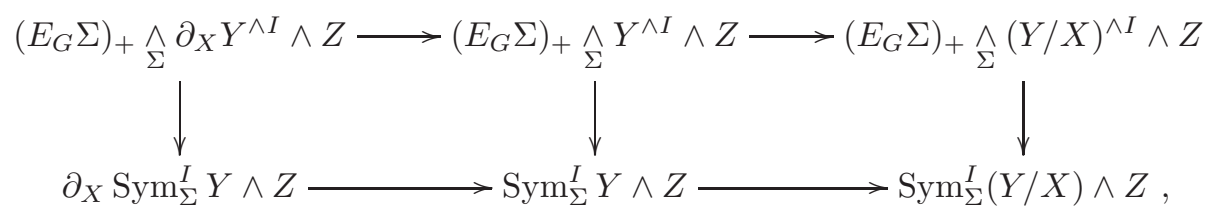

in which $Z$ is any $G$-spectrum. By Proposition B.117 the two right vertical maps are weak equivalences. Since the left horizontal maps are $h$-cofibrations, hence flat, this implies that the left vertical map is a weak equivalences. Taking $Z=S^{0}$ gives the weak equivalence of the vertical arrows in the statement of Proposition B.109. Letting $Z$ vary through a weak equivalence and using the fact that cofibrant objects are flat gives the flatness assertion. By what we have already proved, when $X \rightarrow$ $Y$ is a weak equivalence the vertical and top arrows in the left square are weak equivalences, hence so is the bottom left map. This completes the proof.

Proof of Proposition B.113: Suppose that $X \rightarrow Y$ is a weak equivalence of cofibrant objects, and consider the diagram

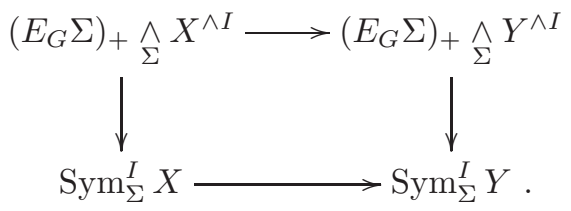

The vertical maps are weak equivalences by Proposition B.117. The top horizontal map is a weak equivalence by Lemma B.115 (applied to, say, the map $(* \rightarrow X) \rightarrow$ $(* \rightarrow Y))$. The bottom map is therefore a weak equivalence.

B.6.2. Iterated indexed and symmetric powers. In our analysis of the norms of commutative rings in $\S \mathrm{B} .8$ we will encounter iterated indexed smash products and symmetric powers. These work out just to be other indexed smash or symmetric powers. The point of this section is to spell this out.

Suppose that $I$ and $J$ are $G$-sets and that $X$ is an equivariant $I \times J$-diagram. The factorization

$$
I \times J \rightarrow J \rightarrow \mathrm{pt}
$$

gives an isomorphism

$$
\left(X^{\wedge I}\right)^{\wedge J} \approx X^{\wedge(I \times J)},
$$

in which $X^{\wedge I}$ is shorthand for $p_{*}^{\wedge} X$ with $p: I \times J \rightarrow J$ the projection mapping. Applying this to the arrow category we get an isomorphism of the corner map

$$
\partial_{X} Y^{\wedge(I \times J)} \rightarrow X^{\wedge(I \times J)}
$$


with the iterated corner map

$$
\partial_{W} Z^{\wedge J} \rightarrow Z^{\wedge J}
$$

in which $W \rightarrow Z$ is the map

$$
\partial_{X} Y^{\wedge I} \rightarrow Y^{\wedge I}
$$

There is also a version with symmetric powers. Suppose in addition that $\Sigma \subset \Sigma_{I}$ is a $G$-stable subgroup. Then the action of $\Sigma^{J}$ on $I \times J$ by

$$
\phi \cdot(i, j)=(\phi(j) \cdot i, j)
$$

is $G$-stable, making $J \times I$ into a $\Sigma^{J} \rtimes G$-set, and the projection map $I \times J \rightarrow J$ equivariant, with $\Sigma^{J} \rtimes G$ acting on $J$ through $G$. When $X$ is a $\Sigma^{J} \rtimes G$-equivariant $J \times I$-diagram, the isomorphism (B.123) is $\Sigma^{J} \rtimes G$-equivariant. Passing to orbits gives an isomorphism of $G$-spectra

$$
\left(\operatorname{Sym}_{\Sigma}^{I} X\right)^{\wedge J} \approx \operatorname{Sym}_{\Sigma^{J}}^{I \times J} X .
$$

By working in the arrow category we get an isomorphism of the corner map

$$
\partial_{X} \operatorname{Sym}_{\Sigma^{J}}^{I \times J} Y \rightarrow \operatorname{Sym}_{\Sigma^{J}}^{I \times J} Y
$$

with the iterated indexed corner map

$$
\partial_{W} Z^{\wedge J} \rightarrow Z^{\wedge J}
$$

in which $W \rightarrow Z$ is the map

$$
\partial_{X} \operatorname{Sym}_{\Sigma}^{I} Y \rightarrow \operatorname{Sym}_{\Sigma}^{I} Y .
$$

Our analysis of the homotopy properties of symmetric powers depended on a convenient cofibrant approximation. Let $E_{G} \Sigma$ be a universal $G$-equivariant $\Sigma$ space. The above discussion leads to an isomorphism

$$
\left(E_{G} \Sigma_{+}{ }_{\Sigma} X^{\wedge I}\right)^{\wedge J} \approx E_{G} \Sigma_{+}^{J}{ }_{\Sigma^{J}} X^{\wedge(I \times J)},
$$

and an identification of the corner map

$$
\partial_{\tilde{W}} \tilde{Z}^{\wedge J} \rightarrow \tilde{Z}^{\wedge J}
$$

in which $\tilde{W} \rightarrow \tilde{Z}$ is the map

$$
E_{G} \Sigma_{+} \wedge \wedge_{\Sigma}\left(\partial_{X} Y^{\wedge I} \rightarrow Y^{\wedge I}\right)
$$

with

$$
\left(E_{G} \Sigma^{J}\right)+{ }_{\Sigma^{J}}\left(\partial_{X} Y^{\wedge(I \times J)} \rightarrow Y^{\wedge(I \times J)}\right)
$$

To reduce this expression to one we have already considered we need to know that $E_{G} \Sigma^{J}$ is a universal equivariant $\Sigma^{J}$-space.

Lemma B.126. Let $J$ be a finite $G$-set. If $E_{G} \Sigma$ is an equivariant universal $\Sigma$-space then, under the product action, $\left(E_{G} \Sigma\right)^{J}$ is an equivariant universal $\Sigma^{J}$-space.

Proof: The functor $T \mapsto T^{J}$ (from $\Sigma \rtimes G$-spaces to $\Sigma^{J} \rtimes G$-spaces) has a left adjoint. To describe it, let $M$ be the set $\Sigma \rtimes G \times J$ and define a left action of $\Sigma \rtimes G$ by the product of the translation action on $\Sigma \rtimes G$ and the action of $G$ on $J$. There is a commuting right $\Sigma^{J} \rtimes G$-action

$$
(\Sigma \rtimes G \times J) \times\left(\Sigma^{J} \rtimes G\right) \rightarrow \Sigma \rtimes G \times J .
$$


whose component in the second factor is just the projection, and in the first factor is composed of the evaluation map

$$
J \times \Sigma^{J} \rtimes G \rightarrow \Sigma \rtimes G
$$

and the right action of $\Sigma \rtimes G$ on itself. The functor $T \mapsto T^{J}$ can be identified with

$$
\operatorname{hom}_{\Sigma \rtimes G}(M, T)
$$

and so its left adjoint is given by

$$
S \mapsto M \underset{\Sigma^{J} \rtimes G}{\times} S .
$$

Breaking $M$ into right $\Sigma^{J} \rtimes G$-orbits gives the decomposition

$$
M \underset{\Sigma^{J} \rtimes G}{\times} S=\coprod_{j \in J} S / \Sigma^{J-\{j\}} .
$$

In this latter expression, the action of $t \in \Sigma$ on $s \in S / \Sigma^{J-\{j\}}$ can be computed as the orbit class of $\sigma s$, where $\sigma \in \Sigma^{J}$ is any element with $\sigma(j)=t$. For example, the entire $\Sigma$-action can be computed by restricting to the diagonal subgroup of $\Sigma^{J}$.

Observe that a $\Sigma^{J} \rtimes G$-set $S$ is $\Sigma^{J}$-free if and only if $M \underset{\Sigma^{J} \rtimes G}{\times} S$ is $\Sigma$-free. Clearly if $S$ is $\Sigma^{J}$-free then for each $j \in J, S / \Sigma^{J-\{j\}}$ is $\Sigma$-free. On the other hand if $\sigma \in \Sigma^{J}$ is a non-identity element fixing $s \in S$, then there is a $j \in J$, with $\sigma(j)$ not the identity element. For this $j$ we have $\sigma(j) \cdot \Sigma^{J-\{j\}} s=\Sigma^{J-\{j\}} s$.

Now to the proof. Let $S$ be a finite $\Sigma^{J} \rtimes G$-set. We need to show that the space of $\Sigma^{J} \rtimes G$-maps

$$
S \rightarrow E_{G} \Sigma^{J}
$$

is empty or contractible depending on whether or not $S$ has a point fixed by a non identity element of $\Sigma^{J}$. By adjunction, this space can be identified with the space of $\Sigma \rtimes G$-maps from

$$
M \underset{\Sigma^{J} \rtimes G}{\times} S \rightarrow E_{G} \Sigma,
$$

and so the result follows from the observation above.

We will be interested in the following case. Suppose that $X \rightarrow Y$ is a cofibration of cofibrant $\Sigma^{J} \rtimes G$-equivariant $I \times J$-diagrams. By Lemma B.126 and Proposition B.109, in the diagram

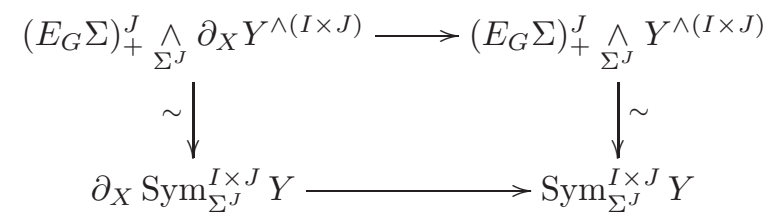

every object is flat, the top row is a cofibration of cofibrant objects, the bottom row is an $h$-cofibration, and the vertical maps are weak equivalences and remain so after smashing with any spectrum. The same conclusion therefore holds for the corresponding diagram of iterated indexed (symmetric) powers

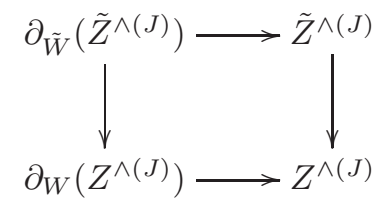


in which

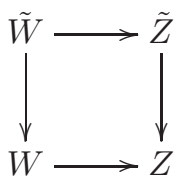

is the diagram

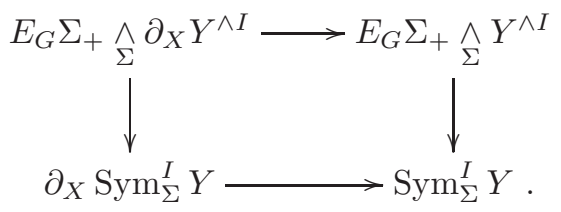

Working fiberwise leads to an analogous result about the indexed smash product along a map $q: J \rightarrow K$ of finite $G$-sets. It plays an important role in our analysis of the homotopy properties of the norms of commutative rings. Aside from the map $J \rightarrow K$ of finite $G$-sets, the situation is the same as what we have been discussing in this section. We have fixed a finite $G$-set $I$, a $G$-stable subgroup $\Sigma \subset \Sigma_{I}$, and a universal $G$-equivariant $\Sigma$-space $E_{G} \Sigma$.

Proposition B.127. Let $X \rightarrow Y$ be a cofibration of cofibrant $\Sigma^{J} \rtimes G$-equivariant $I \times J$-diagrams and write

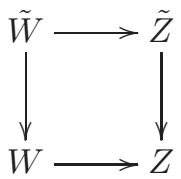

for the diagram

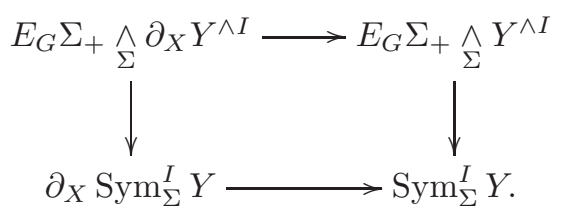

In the $G$-equivariant $K$-diagram of corner maps

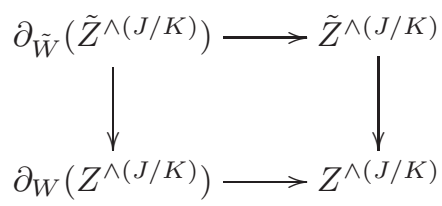

every object is flat, the vertical maps are weak equivalences after smashing with any object, the upper map is a cofibration of cofibrant objects and the lower map is an $h$-cofibration. The horizontal maps are weak equivalences if $X \rightarrow Y$ is.

Remark B.128. The actual hypothesis on $X \rightarrow Y$ required for the fiberwise argument is that for each $k \in K$, the map $X \rightarrow Y$ is a cofibration of $\Sigma^{J_{k}} \rtimes G_{k}$-equivariant $I \times J_{k}$-diagram, where $J_{k} \subset J$ is the inverse image of $k$, and $G_{k}$ is the stabilizer of $k$. For the sake of a cleaner statement we have made the slightly stronger assumption that it is a cofibration of cofibrant $\Sigma^{J} \rtimes G$-equivariant $I \times J$-diagrams. That this implies the "fiberwise" hypothesis is a consequence of Proposition B.75. 
Remark B.129. As in Remark B.112, Proposition B.127 applies to the situation which $X \rightarrow Y$ is a cofibration of cofibrant $G$-equivariant $J$-diagrams, regarded as a $\Sigma \rtimes G$-equivariant $I \times J$ diagram by pulling back along the projection mappings $\Sigma \rtimes G \rightarrow G$ and $I \times J \rightarrow J$.

B.7. Rings and modules. Aside from the alteration in model structure, the following is stated as [53, Theorem III.8.1]. The proof depends on our analysis of symmetric powers, which, as mentioned in Remark B.120, makes essential use of the norm.

Proposition B.130. The forgetful functor

$$
\mathrm{Comm}^{G} \rightarrow \mathcal{S}^{G}
$$

creates a topological model category structure on $\mathbf{C o m m}^{G}$ in which the fibrations and weak equivalences in $\mathbf{C o m m}^{G}$ are the maps that are fibrations and weak equivalences in $\mathcal{S}^{G}$.

Proof: Most of the proof is formal. One takes as generating cofibrations the maps Sym $A \rightarrow \operatorname{Sym} B$ where $A \rightarrow B \in \mathcal{A}_{\text {cof }}$ and generating acyclic cofibrations the maps $\operatorname{Sym} A \rightarrow \operatorname{Sym} B$ with $A \rightarrow B \in \mathcal{B}_{\text {acyclic }}$. The only real point to check is that if

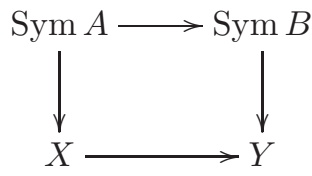

is a pushout diagram in which $A \rightarrow B$ is a generating acyclic cofibration, then $X \rightarrow Y$ is a weak equivalence. That is contained in Lemma B.132 below. The rest of the proof is left to the reader.

Lemma B.132. Suppose that $A \rightarrow B$ is a map of $G$-spectra, and

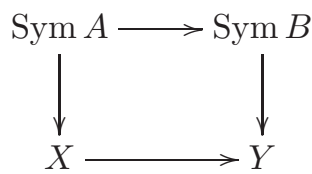

is a pushout diagram of equivariant commutative rings. If $A \rightarrow B$ is an acyclic cofibration of cofibrant objects, then $X \rightarrow Y$ is a weak equivalence.

The proof of Lemma B.132 involves a filtration of $Y$ by $X$-modules which we will use again in $\S$ B.8. For a map $A \rightarrow B$ of $G$-spectra define

$$
\mathrm{fil}_{m} \operatorname{Sym} B=\bigvee_{n} \mathrm{fil}_{m} \operatorname{Sym}^{n} B
$$

where the $\operatorname{fil}_{m} \operatorname{Sym}^{n} B$ is obtained from the filtration described in $\S$ A.3.4 by passing to $\Sigma_{n}$-orbits, and fits into a pushout square

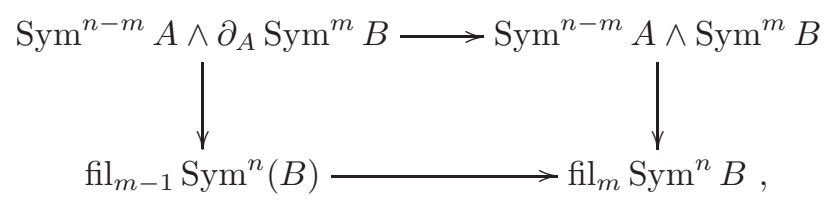


with

$$
\partial_{A} \operatorname{Sym}^{m} B=\left(\partial_{A} B^{\wedge m}\right) / \Sigma_{m} .
$$

Wedging over $n$ one sees that the $\operatorname{fil}_{m} B$ are Sym $A$-submodules, and that there is a pushout square of $A$-modules

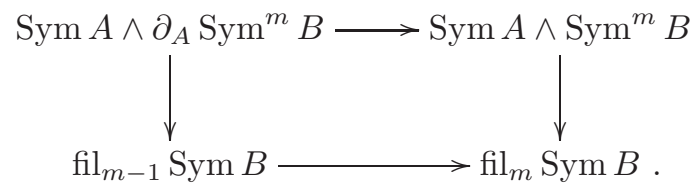

If a map $X \rightarrow Y$ of commutative rings fits into a pushout diagram

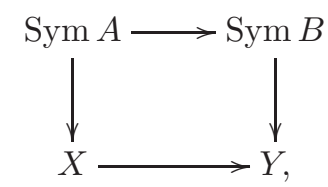

we can define a filtration of $Y$ by $X$-modules by

$$
\mathrm{fil}_{m} Y=X \underset{\operatorname{Sym}_{A}}{\wedge} \mathrm{fil}_{m} \operatorname{Sym} B .
$$

Evidently these $\operatorname{fil}_{m} Y$ are related by the pushout square of $X$-modules

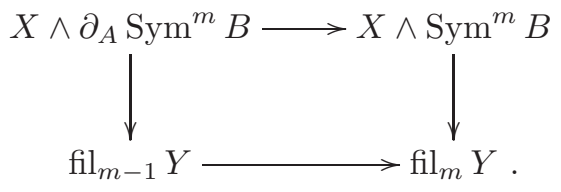

Proof of Lemma B.132: We use the filtration just described. In the diagram (B.133), if $A \rightarrow B$ is an acyclic cofibration between cofibrant objects, then

$$
\partial_{A} \operatorname{Sym}^{m} B \rightarrow \operatorname{Sym}^{m} B
$$

is a weak equivalence and an $h$-cofibration of flat spectra by Proposition B.109. It follows that the bottom map is a weak equivalence.

Corollary B.134. For $H \subset G$, the adjoint functors

$$
\operatorname{Comm}^{H} \leftrightarrows \operatorname{Comm}^{G}
$$

form a Quillen pair.

Proof: The restriction functor obviously preserves the classes of fibrations and weak equivalences.

Corollary B.135. The norm functor on commutative algebras

$$
N_{H}^{G}: \mathbf{C o m m}^{H} \rightarrow \mathbf{C o m m}^{G}
$$

is a left Quillen functor. It preserves the classes of cofibrations and acyclic cofibrations, hence weak equivalences between cofibrant objects. 
Proof: This is immediate from Corollary B.134 and Proposition A.56. The assertion about weak equivalences is Ken Brown's Lemma (see, for example [34, Lemma 1.1.12]).

The category $\mathcal{M}_{R}$ of left modules over an equivariant associative algebra $R$ as defined in $\S A .2 .7$. As pointed out there, when $R$ is commutative, a left $R$-module can be regarded as a right $R$-module, and $\mathcal{M}_{R}$ becomes a symmetric monoidal category under the operation

$$
M \underset{R}{\wedge} N
$$

The following result is a consequence of Propositions B.76, B.77 and [76, Theorem 4.1]. Except for the slight change of model structure, it is [53, Theorem III.7.6].

Proposition B.137. The forgetful functor

$$
\mathcal{M}_{R} \rightarrow \mathcal{S}^{G}
$$

creates a model structure on the category $\mathcal{M}_{R}$ in which the fibrations and weak equivalences are the maps which become fibrations and weak equivalences in $\mathcal{S}^{G}$. When $R$ is commutative, the operation (B.136) satisfies the pushout product and monoid axioms making $\mathcal{M}_{R}$ into a symmetric monoidal model category.

Though not explicitly stated, the following formal result was surely known to the authors of [76] (see the proof of [76, Theorem 4.3].)

Corollary B.138. Let $f: R \rightarrow S$ be a map of equivariant associative algebras. The functors

$$
S \underset{R}{\wedge}(-): \mathcal{M}_{R} \leftrightarrows \mathcal{M}_{S}: U
$$

given by restriction and extension of scalars form a Quillen pair. If $S$ is cofibrant as a left $R$-module, then the restriction functor is also a left Quillen functor.

Proof: Proposition B.137 implies that the restriction functor preserves fibrations and acyclic fibrations. This gives the first assertion. The second follows from the fact that the restriction functor preserves colimits, and the consequence of Proposition B.137 that the generating (acyclic) cofibrations for $\mathcal{M}_{S}$ are formed as the smash product of $S$ with the generating (acyclic) cofibrations for $\mathcal{S}^{G}$.

The following result is [53, Proposition III.7.7]. Using the fact that $h$-cofibrations are flat, the proof reduces to checking the case $M=G_{+}{ }_{H} S^{-V} \wedge R$, which is Proposition B.58.

Proposition B.139. Suppose that $R$ is an associative algebra, and $M$ is a cofibrant right $R$-module. The functor $M \wedge_{R}(-)$ preserves weak equivalences.

In other words, the functor $M \underset{R}{\wedge}(-)$ is flat if $M$ is cofibrant, and so need not be derived.

Corollary B.140. Suppose that $R$ is an associative algebra, $M$ a cofibrant right $R$-module. If $N \rightarrow N^{\prime}$ a map of left $R$-modules whose underlying map of spectra is an $h$-cofibration, then the sequence

$$
M \underset{R}{\wedge} N \rightarrow M \underset{R}{\wedge} N^{\prime} \rightarrow M \underset{R}{\wedge}\left(N^{\prime} / N\right)
$$

is weakly equivalent to a cofibration sequence. 
Note that the assumption is not that $N \rightarrow N^{\prime}$ is an $h$-cofibration in the category of left $R$-modules. In that case the result would not require any hypothesis on $M$.

Proof: We must show that the map from the mapping cone of

$$
M \underset{R}{\wedge} N \rightarrow M \underset{R}{\wedge} N^{\prime}
$$

to $M \stackrel{\wedge}{\wedge}\left(N^{\prime} / N\right)$ is a weak equivalence. The mapping cone of (B.141) is isomorphic to

$$
M \underset{R}{\wedge}\left(N^{\prime} \cup C N\right),
$$

and the spectrum underlying the $R$-module mapping cone $N^{\prime} \cup N$ is the mapping cone formed in spectra. Since $N \rightarrow N^{\prime}$ is an $h$-cofibration, the map $N^{\prime} \cup C N \rightarrow$ $N^{\prime} / N$ is a weak equivalence (Proposition B.20). The result now follows from Proposition B.139.

Corollary B.140 can be used to show that many constructions derived from the formation of monomial ideals have good homotopy theoretic properties. It is used in $\S 2.4 .3$ and in $\S 6.1$. In those cases the map of spectra underlying $N \rightarrow N^{\prime}$ is the inclusion of a wedge summand, and so obviously an $h$-cofibration.

\section{B.8. Indexed smash products of commutative rings.}

B.8.1. Description of the problem. Proposition B.105 asserts that the indexed smash product functor

$$
(-)^{\wedge J}: \mathcal{S}^{\mathcal{B}_{J} G} \rightarrow \mathcal{S}^{G}
$$

has a left derived functor

$$
(-)^{\text {永J}}: \operatorname{ho} \mathcal{S}^{\mathcal{B}_{J} G} \rightarrow \operatorname{hos}^{G}
$$

which can be computed by applying the norm to a cofibrant approximation. We also know from Corollary B.134 (and the fact that coproducts of weak equivalences are weak equivalences) that the restriction functor and its left adjoint form a Quillen pair

$$
p_{!}: \operatorname{comm} \mathcal{S}^{\mathcal{B}_{J} G} \leftrightarrows \operatorname{comm} \mathcal{S}^{G}: p^{*}
$$

Furthermore, the following diagram commutes in which the vertical functors are the forgetful functors (Corollary A.56)

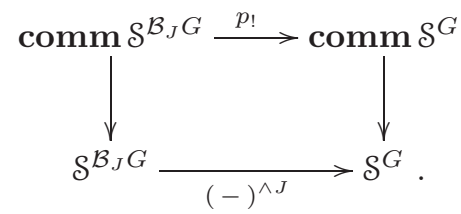

However, what we really want is the commutativity of the following diagram

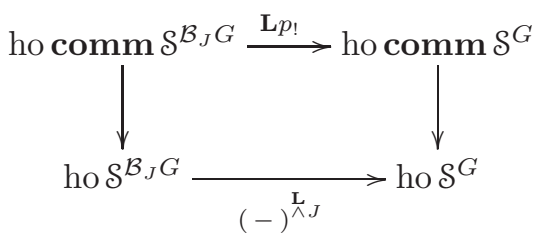


in which the vertical maps are the forgetful functors (which are homotopical, so don't need to be derived), and the horizontal arrows are the left derived functors indicated. The point of this section is to establish this.

To clarify the issue, suppose that $R \in \operatorname{comm} \mathcal{S}^{\mathcal{B}_{J} G}$ is a cofibrant $J$-diagram of commutative rings. Let $\tilde{R} \rightarrow R$ be a cofibrant approximation of the underlying $J$-diagram of spectra. What needs to be checked is that the map

$$
(\tilde{R})^{\wedge J} \rightarrow(R)^{\wedge J}
$$

is a weak equivalence. The proof involves an elaboration of the notion of flatness. To motivate it we describe a bit of the argument.

The main point in the proof is to investigate the situation of a pushout diagram of equivariant $J$-diagrams of commutative rings

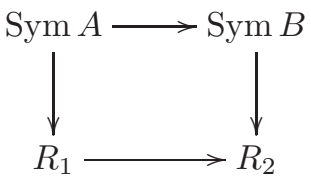

in which the top row is constructed by applying the symmetric algebra functor Sym to a generating cofibration $A \rightarrow B$, and in which one knows that the map (B.142) is a weak equivalence for $R=R_{1}$. One would like to conclude that (B.142) is a weak equivalence for $R=R_{2}$.

To pass from $R_{1}$ to $R_{2}$ we use the $R_{1}$-module filtration described after the statement of Lemma B.132, whose stages fit into a pushout square

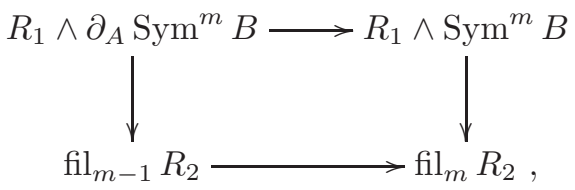

where

$$
\partial_{A} \operatorname{Sym}^{m} B=\left(\partial_{A} B^{\wedge m}\right) / \Sigma_{m} .
$$

The filtration of $\oint$ A.3.4 mediates between $\left(\text { fil }_{m-1} R_{2}\right)^{\wedge J}$ and $\left(\mathrm{fil}_{m} R_{2}\right)^{\wedge J}$ by another sequence of pushout squares. The upper right hand corner of a typical stage is an indexed wedge of terms of the form

$$
\left(\text { fil }_{m-1} R_{2}\right)^{\wedge J_{0}} \wedge\left(R_{1} \wedge \operatorname{Sym}^{m} B\right)^{\wedge J_{1}},
$$

indexed by the set-theoretic decompositions $J=J_{0} \amalg J_{1}$.

We need to know two things about this expression. One is that the left derived functor of its formation (in all variables) is computed in terms of the expression itself, and the other is that formation of each of the pushout squares we encounter is homotopical. Motivated by this we are led to consider a technical condition slightly stronger than the requirement that (B.142) be a weak equivalence. That is the subject of the next section.

B.8.2. Very flat diagrams. As in $§ B .6 .2$, to make the diagrams more readable we will use the notation

$$
X^{\wedge(K / L)}=q_{*}^{\wedge} X
$$

for the indexed smash product along a map $q: K \rightarrow L$ of finite $G$-sets. 
Definition B.145. An equivariant $J$-diagram $X$ very flat if it has the following property: for every cofibrant approximation $\tilde{X} \rightarrow X$, every diagram of finite $G$-sets

$$
J \stackrel{p}{\leftarrow} K \stackrel{q}{\rightarrow} L,
$$

and every weak equivalence of equivariant $L$-diagrams $\tilde{Z} \rightarrow Z$, the map

$$
\left(p^{*} \tilde{X}\right)^{\wedge(K / L)} \wedge \tilde{Z} \rightarrow\left(p^{*} X\right)^{\wedge(K / L)} \wedge Z
$$

is a weak equivalence.

Our main goal is to establish the following result.

Proposition B.147. If $R \in \mathcal{S}^{\mathcal{B}_{J} G}$ is cofibrant commutative ring, then the equivariant $J$-diagram of spectra underlying $R$ is very flat.

The condition that $R$ be very flat certainly implies that (B.142) is a weak equivalence. Proposition B.147 therefore implies

Corollary B.148. The following diagram of left derived functors commutes up to natural isomorphism

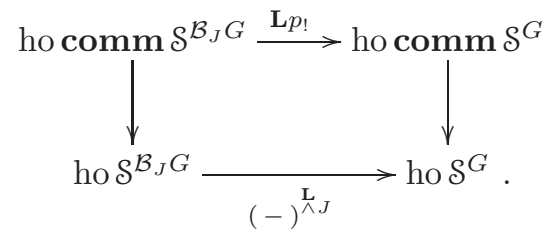

Remark B.149. Since identity maps are weak equivalences, the condition of being very flat implies that every arrow in the diagram

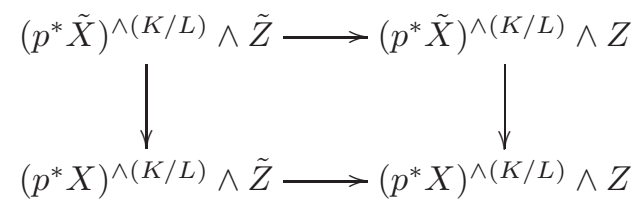

is a weak equivalence. In particular it implies that $\left(p^{*} X\right)^{\wedge(K / L)}$ is flat.

Remark B.150. Since $\tilde{X}^{\wedge(K / L)}$ is cofibrant (Proposition B.89), and cofibrant objects are flat (Proposition B.58), the top arrow in the above diagram is always a weak equivalence. It therefore suffices to check the very flat condition when $\tilde{Z} \rightarrow Z$ is the identity map.

Remark B.151. If (B.146) is a weak equivalence for one cofibrant approximation it is a weak equivalence for any cofibrant approximation. It therefore suffices to check the "very flat" condition for a single cofibrant approximation $\tilde{X} \rightarrow X$.

Lemma B.152. Arbitrary wedges of very flat spectra are very flat. Smash products of very flat spectra are very flat. Filtered colimits of very flat $G$-diagrams along $h$ cofibrations are very flat.

Proof: The first assertion follows easily from the distributive law, and the fact that the formation of indexed wedges is homotopical. The second follows from the 
fact that the formation of indexed smash products is symmetric monoidal. The third makes use of Proposition A.69. The details are left to the reader.

Example B.153. Here is one motivation for the definition of "very flat." Suppose we are given a pushout square of equivariant $J$-diagrams

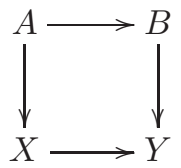

and we are interested in the filtration of $Y^{\wedge(K / L)}$ described in $\S$ A.3.4, whose stages are related by pushout squares

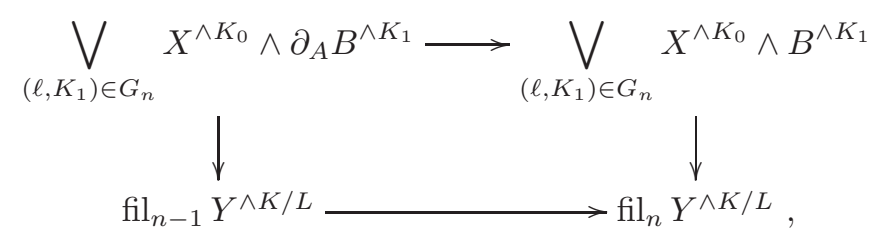

where $G_{n}=G_{n}(K / L)$ is the $G$-set of pairs $\left(\ell, K_{1}\right)$ with $\ell \in L$ and $K_{1} \subset q^{-1}(\ell)$ a subset of cardinality $n$, and the map $G_{n} \rightarrow L$ sends $\left(\ell, K_{1}\right)$ to $\ell$. For $\left(\ell, K_{1}\right) \in G_{n}$ we have written $K_{0}$ to denote the complement of $K_{1}$ in $q^{-1}(\ell)$.

The condition that $B$ be very flat gives some control over the upper right term. To see this let $V_{n}=V_{n}(K / L)$ be the set of triples $\left(\ell, K_{1}, k\right)$ with $\left(\ell, K_{1}\right) \in G_{n}$ and $k \in K_{1}$. We define maps

$$
J \stackrel{f}{\leftarrow} V_{n} \stackrel{g}{\rightarrow} G_{n}
$$

by

$$
\begin{aligned}
& f\left(\ell, K_{1}, k\right)=q(k) \\
& g\left(\ell, K_{1}, k\right)=\left(\ell, K_{1}\right) .
\end{aligned}
$$

The spectra $X^{\wedge K_{0}}$ form an equivariant $G_{n}$-diagram, which we denote $Z$. The $B^{\wedge K_{1}}$ are the constituents of $\left(f^{*} B\right)^{\wedge\left(V_{n} / G_{n}\right)}$, and so the indexed wedge occurring in the pushout square is

$$
\bigvee_{G_{n}} Z \wedge\left(f^{*} B\right)^{\wedge\left(V_{n} / G_{n}\right)} .
$$

Since the formation of indexed wedges is homotopical, its homotopy properties come down to understanding the homotopy properties of the equivariant $G_{n}$-diagram $Z \wedge f^{*} B^{\wedge\left(V_{n} / G_{n}\right)}$, some of which are specified by the condition that $B$ be very flat.

By replacing the category of equivariant $J$-diagrams with its arrow category, we arrive at the notion of a very flat object of $\mathcal{S}_{1}^{\mathcal{B}_{J} G}$. The formal properties of being very flat persist in this context, and in particular the analogues of Remarks B.149, B.150, B.151 and Lemma B.152 hold.

To get a feel for the more particular aspects of very flat arrows, suppose that $(A \rightarrow B)$ is an object of $\mathcal{S}_{1}^{\mathcal{B}_{J} G}$, and $(\tilde{A} \rightarrow \tilde{B})$ is a cofibrant approximation. Consider a weak equivalence of the form

$$
(\tilde{X} \rightarrow *) \rightarrow(X \rightarrow *)
$$


In this case the very flat condition becomes that

$$
\left(p^{*}(\tilde{B} / \tilde{A})^{\wedge(K / L)} \wedge \tilde{X} \rightarrow *\right) \rightarrow\left(p^{*}(B / A)^{\wedge(K / L)} \wedge X \rightarrow *\right)
$$

is a weak equivalence. This is so if and only if $B / A$ is very flat.

Next consider a weak equivalence of the form

$$
(* \rightarrow \tilde{X}) \rightarrow(* \rightarrow X) .
$$

The very flat condition in this case is that

$$
\begin{aligned}
\left(\partial_{p^{*} \tilde{A}} p^{*} \tilde{B}^{\wedge(K / L)} \wedge \tilde{X} \rightarrow p^{*} \tilde{B}^{\wedge(K / L)}\right. & \wedge \tilde{X}) \\
& \rightarrow\left(\partial_{p^{*} A} p^{*} B^{\wedge(K / L)} \wedge X \rightarrow p^{*} B^{\wedge(K / L)} \wedge X\right)
\end{aligned}
$$

is a weak equivalence. This holds if and only if $B$ is very flat and $(A \rightarrow B)$ satisfies the condition that

$$
\partial_{p^{*} \tilde{A}} p^{*} \tilde{B}^{\wedge(K / L)} \wedge \tilde{X} \rightarrow \partial_{p^{*} A} p^{*} B^{\wedge(K / L)} \wedge X
$$

is a weak equivalence. If we happen to know that the indexed corner maps

$$
\partial_{p^{*} \tilde{A}} p^{*} \tilde{B}^{\wedge(K / L)} \rightarrow \tilde{B}^{\wedge(K / L)}
$$

and

$$
\partial_{p^{*} A} p^{*} B^{\wedge(K / L)} \rightarrow B^{\wedge(K / L)}
$$

are $h$-cofibrations then the leftmost horizontal maps in

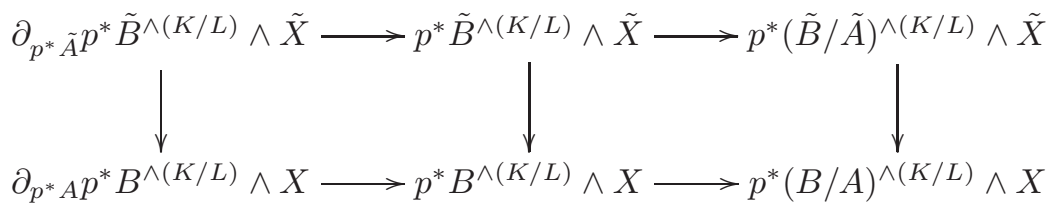

are $h$-cofibrations, hence flat. Thus the middle and left vertical arrows are weak equivalences if and only if the middle and right vertical arrows are, or in other words if and only if both $B$ and $B / A$ are very flat. So in the presence of the condition above, a necessary condition that $(A \rightarrow B)$ be a very flat arrow is that $B$ and $B / A$ are very flat. This turns out to be sufficient. We single out the condition.

Condition B.156. For every $J \stackrel{p}{\leftarrow} K \stackrel{q}{\rightarrow} L$ the corner map

$$
\partial_{p^{*} A}\left(p^{*} B\right)^{\wedge(K / L)} \rightarrow\left(p^{*} B\right)^{\wedge(K / L)}
$$

is an h-cofibration.

Remark B.157. By Proposition B.96 and the monoid axiom for $\mathcal{S}_{1}^{\mathcal{B}_{L} G}$, a cofibrant object $(A \rightarrow B)$ of $\mathcal{S}_{1}^{\mathcal{B}_{J} G}$ is very flat and satisfies Condition B.156.

Lemma B.158. If $A_{1} \rightarrow A_{2}$ satisfies Condition B.156, and both $A_{1}$ and $A_{2} / A_{1}$ are very flat, then $A=\left(A_{1} \rightarrow A_{2}\right)$ is very flat.

Proof: Fix a diagram of finite $G$-sets

$$
J \stackrel{p}{\leftarrow} K \stackrel{q}{\rightarrow} L
$$


let $\tilde{A}=\left(\tilde{A}_{1} \rightarrow \tilde{A}_{2}\right)$ be a cofibrant approximation to $A=\left(A_{1} \rightarrow A_{2}\right)$, and

$$
\begin{gathered}
\tilde{X} \rightarrow X \\
\tilde{X}=\left(\tilde{X}_{1} \rightarrow \tilde{X}_{2}\right) \\
X=\left(X_{1} \rightarrow X_{2}\right)
\end{gathered}
$$

a weak equivalence in $\mathcal{S}_{1}^{\mathcal{B}_{L} G}$. By Remark B.157, $\tilde{A}$ also satisfies the conditions of the lemma. Let

$$
X^{\prime} \rightarrow X \rightarrow X^{\prime \prime}
$$

be the sequence

$$
\left(* \rightarrow X_{2}\right) \rightarrow\left(X_{1} \rightarrow X_{2}\right) \rightarrow\left(X_{1} \rightarrow *\right) .
$$

and $\tilde{X}^{\prime} \rightarrow \tilde{X} \rightarrow \tilde{X}^{\prime}$ the analogous sequence for $\tilde{X}$. The maps $X^{\prime} \rightarrow X$ and $\tilde{X}^{\prime} \rightarrow \tilde{X}$ are not $h$-cofibrations, but they are so objectwise, and hence flat.

Consider the diagram

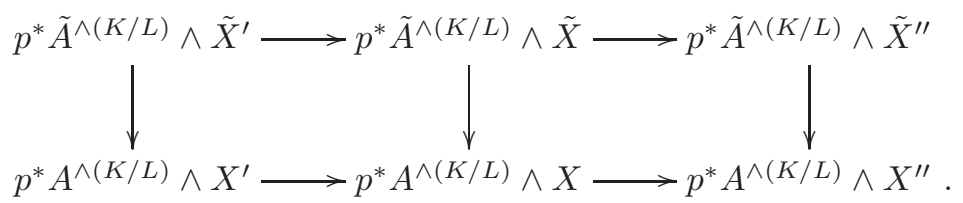

Our aim is to show that the middle vertical map is a weak equivalence.

The first step is to show that the left horizontal maps are flat. This reduces us to checking that the left and right vertical maps are weak equivalences. For this, let's examine the bottom left horizontal map in more detail. It is given by

$$
\left(\partial_{p^{*} A_{1}} p^{*} A_{2}^{\wedge(K / L)} \wedge X_{2} \rightarrow p^{*} A_{2}^{\wedge(K / L)} \wedge X_{2}\right) \rightarrow\left(C \rightarrow p^{*} A_{2}^{\wedge(K / L)} \wedge X_{2}\right)
$$

in which $C$ is defined by the pushout diagram

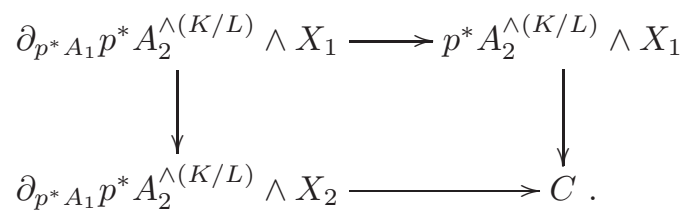

When $A_{1} \rightarrow A_{2}$ satisfies Condition B.156 the top map in (B.161) is an $h$-cofibration, hence so is the bottom map. This means that (B.160) is an objectwise $h$-cofibration, and so flat. Since $\tilde{A}_{1} \rightarrow \tilde{A}_{2}$ also satisfies Condition B.156 the upper left horizontal map in (B.159) is also flat. Thus we are reduced to checking that the maps

$$
\begin{gathered}
p^{*} \tilde{A}^{\wedge(K / L)} \wedge \tilde{X}^{\prime} \rightarrow p^{*} A^{(\wedge K / L)} \wedge X^{\prime} \\
p^{*} \tilde{A}^{\wedge(K / L)} \wedge \tilde{X}^{\prime \prime} \rightarrow p^{*} A^{(\wedge K / L)} \wedge X^{\prime \prime}
\end{gathered}
$$

are weak equivalences. As described above, this fact for the second map follows from the assumption that $A_{2} / A_{1}$ is very flat. The assertion in the case of the first map is that the middle and left vertical arrows in

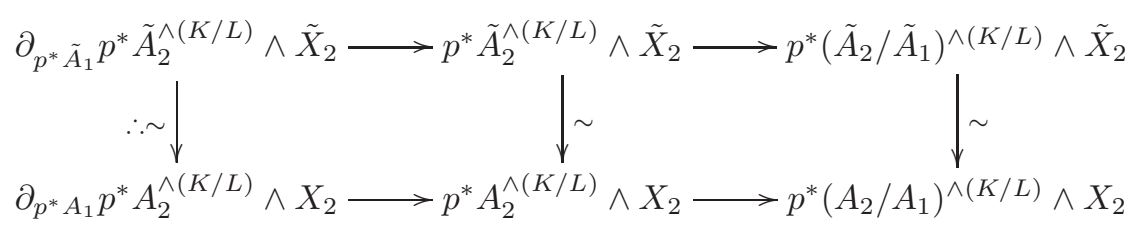


are weak equivalences. Since $A_{2}$ and $A_{2} / A_{1}$ are very flat, the middle and right vertical maps are weak equivalences. Condition B.156 shows that the left horizontal maps are $h$-cofibrations, hence flat. It follows that the left vertical map is a weak equivalence.

We can now establish an important technical fact used in the proof of Proposition B.147.

Lemma B.162. Suppose that $A \rightarrow B$ is a cofibrant object of $\mathcal{S}_{1}^{\mathcal{B}_{J} G}, I$ is a $G$-set and $\Sigma \subset \Sigma_{I}$ a $G$-stable subgroup. Then

$$
\operatorname{Sym}_{\Sigma}^{I}(A \rightarrow B)=\left(\partial_{A} \operatorname{Sym}_{\Sigma}^{I} B \rightarrow \operatorname{Sym}_{\Sigma}^{I} B\right)
$$

is very flat.

Proof: Proposition B.127 implies that in this situation the map $\operatorname{Sym}_{\Sigma}^{I}(A \rightarrow$ $B$ ) satisfies Condition B.156, and that for every cofibrant $B$, $\operatorname{Sym}_{\Sigma}^{I} B$ is very flat (so both $\operatorname{Sym}_{\Sigma}^{I} B$ and $\operatorname{Sym}_{\Sigma}^{I}(B / A)$ are very flat). The result then follows from Lemma B.158.

Example B.163. Continuing with Example B.153, the top map in (B.154) arises naturally in the arrow category as

$$
\bigvee_{G_{n}(K / L)} Z \wedge\left(p^{*}(A \rightarrow B)^{\wedge(K / L)}\right),
$$

where $Z$ is the identity arrow of the diagram $X^{\wedge J_{0}}$. Since the formation of indexed wedges is homotopical, the information in the homotopy type of this expression is contained in the $G_{n}(K / L)$-diagram $Z \wedge\left(p^{*}(A \rightarrow B)^{\wedge(K / L)}\right)$. The condition that $(A \rightarrow B)$ be very flat thus specifies good homotopical properties of the top map in (B.154).

Lemma B.164. Consider a pushout square

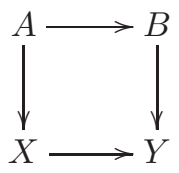

in which $(A \rightarrow B)$ is a very flat object of $\mathcal{S}_{1}^{\mathcal{B}_{J} G}$ satisfying Condition B.156. If $X$ is very flat, then so is $Y$.

Proof: Using the fact that cofibrations are flat, we can arrange things so that the cofibrant approximation $\tilde{Y} \rightarrow Y$ fits into a pushout square

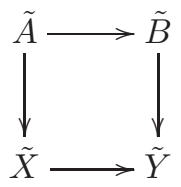

of cofibrant approximations to (B.165), in which $\tilde{A} \rightarrow \tilde{B}$ is a cofibration. We give $\tilde{Y}^{\wedge(K / L)}$ and $Y^{\wedge(K / L)}$ the filtration described in $\S$ A.3.4. We will prove by induction on $n$ that for any weak equivalence $\tilde{Z} \rightarrow Z$ of equivariant $J$-diagrams, the map

$$
\operatorname{fil}_{n} \tilde{Y}^{\wedge K} \wedge \tilde{Z} \rightarrow \operatorname{fil}_{n} Y^{\wedge K} \wedge Z
$$


is a weak equivalence. The case $n=0$ is the assertion that $X$ is very flat, which is true by assumption. For the inductive step, consider the diagram

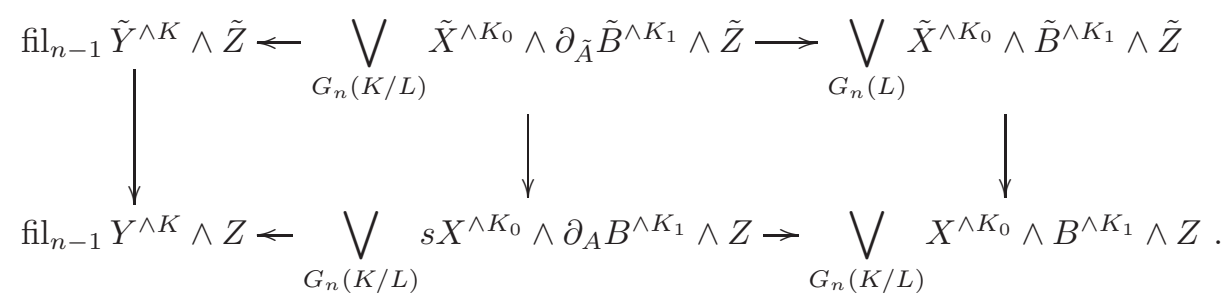

The map from the pushout of the top row to the pushout of the bottom row is (B.167). The rightmost horizontal maps are $h$-cofibrations by Condition B.156. The left vertical map is a weak equivalence by induction, and the other two vertical maps are weak equivalences since $(A \rightarrow B)$ is very flat (Example B.163). The map of pushouts is therefore a weak equivalence since $h$-cofibrations are flat.

B.8.3. Proof of Proposition B.14\%. Since the class of very flat $G$-diagrams is closed under the formation of filtered colimits along $h$-cofibrations (Lemma B.152), it suffices to show that if $A \rightarrow B$ is a generating cofibration in $\mathcal{S}^{\mathcal{B}_{J} G}$,

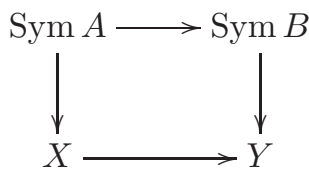

is a pushout square of commutative $J$-algebras, and $X$ is very flat, then $Y$ is very flat. Working fiberwise, the filtration described after the statement of Lemma B.132 gives a filtration of $Y$ by $X$-modules, whose stages are related by the pushout squares

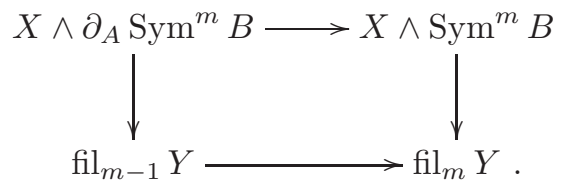

We show by induction on $m$ that each $\operatorname{fil}_{m} Y$ is very flat. Since $\operatorname{fil}_{0} Y=X$, the induction starts. The arrow $\left(\partial_{A} \operatorname{Sym}^{m} B \rightarrow \operatorname{Sym}^{m} B\right)$ is very flat by Lemma B.162. This means that the top row of (B.168) is a very flat arrow, since smash products of very flat objects are very flat (Lemma B.152). This places us in the situation of Lemma B.164 which completes the inductive step.

B.9. The slice tower, symmetric powers and the norm. The main goal of this section is to show that if $R$ is an equivariant commutative ring in $\mathcal{S}_{\geq 0}^{G}$, and $n \geq 0$ is an integer, then the slice section $P^{n} R$ is also an equivariant commutative ring in $\mathcal{S}_{>0}^{G}$. The proof makes use of the technology used to show that cofibrant commutative rings are very flat, and so has been deferred to this appendix. The reader may wish to look through the first three subsections of $\S 4$ for the basic definitions concerning the slice tower.

Our presentation of the slice tower was made in a context where the emphasis was on homotopy theory, and the slice sections $P^{n}$, etc were characterized by homotopy theoretic properties. Here we will be making use of some explicit constructions, and 
some care needs to be taken to ensure that the derived functors we are ultimately interested in can be computed on the objects that arise. Using the fact that indexed smash products of cofibrant objects are cofibrant, and that indexed symmetric powers of cofibrant spectra are flat, one can check that this is indeed the case. We will take as the definition of $P^{n}$ the colimit of the inductive construction described in $\S 4.2$, using the cofibrant approximations $S^{-1} \wedge S^{1} \wedge G_{+}{ }_{H} S^{k \rho_{H}}$ and $S^{-1} \wedge$ $S^{1} \wedge G_{+}{ }_{H} S^{k \rho_{H}-1}$ for the slice cells. This particular choice of $P^{n}$ is homotopical, and the natural map $X \rightarrow P^{n} X$ is a cofibration. Our task will be to show that something functorially weakly equivalent to $P^{n}$ takes commutative rings in $\mathcal{S}_{\geq 0}^{G}$ to commutative rings in $\mathcal{S}_{\geq 0}^{G}$.

We begin with the interaction of the slice filtration with the formation of indexed smash products. As in $\S$ B. 5 we fix a finite $G$-set and work with the homotopy theory of equivariant $J$-diagrams. We define slice cells and the slice filtration in the evident manner, so that the slice filtration on equivariant $J$-diagrams corresponds to the product of slice filtrations on $G_{t}$-spectra under the equivalence

$$
\mathcal{S}^{\mathcal{B}_{J} G} \approx \prod_{t} \mathcal{S}^{G_{t}}
$$

The proposition below follows easily from Proposition 4.13.

Proposition B.169. Suppose that $J$ is a non-empty $G$-set, $X$ is a cofibrant equivariant $J$-diagram, and $n$ is an integer. If each $X_{j}$ is slice $(n-1)$ positive, then the indexed wedge

$$
\bigvee_{j \in J} X_{j}
$$

is slice $(n-1)$ positive.

The next two results make use of the implication

$$
X \geq 0 \text { and } Y \geq k \Longrightarrow X \wedge Y \geq k
$$

proved in $\S 4.3$ (Proposition 4.26).

Proposition B.171. Suppose that $J$ is a non-empty $G$-set, $X$ is a cofibrant equivariant $J$-diagram, and $n \geq 0$ is an integer. If each $X_{j}$ is slice $(n-1)$ positive, then the indexed smash product

$$
\bigwedge_{j \in J} X_{j}
$$

is slice $(n-1)$ positive.

Proof: By induction on $|G|$ we may suppose that $i_{H}^{*} X^{\wedge J}$ is slice $(n-1)$-positive for any proper subgroup $H \subset G$. This implies that $T \wedge X^{\wedge J} \geq n$ if $T$ is any $G$-CW complex built entirely from induced $G$-cells. Since the formation of indexed smash products commutes with filtered colimits, it suffices by Proposition 4.15 to consider a cofibration $A \rightarrow B$ of equivariant $J$-diagrams in which $B / A$ is a wedge of slice cells of dimension greater than $n$, and show that

$$
A^{\wedge J} \geq n \Longrightarrow B^{\wedge J} \geq n
$$


Using the filtration of $\S A .3 .4$ for the identity pushout square

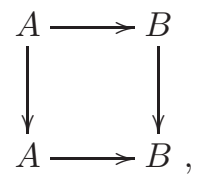

gives a filtration of $B^{\wedge J}$ whose stages fit into cofibration sequences

$$
\text { fil }_{m-1} B^{\wedge J} \rightarrow \text { fil }_{m} B^{\wedge J} \rightarrow \bigvee A^{\wedge J_{0}} \wedge(B / A)^{\wedge J_{1}}
$$

in which the indexing $G$-set for the coproduct is the set of all set theoretic decomposition $J=J_{0} \amalg J_{1}$ with $\left|J_{1}\right|=m$. The implication (B.170) and Proposition B.169 above reduce the claim to showing that if $J_{1} \neq \emptyset$, then $(B / A)^{\wedge J_{1}}$ (regarded as an equivariant spectrum for the stabilizer of $\left.J_{1}\right)$ is slice $(n-1)$-positive. In other words, it suffices to prove the proposition when $X$ is a wedge of slice cells of dimension greater than or equal to $n$.

Making use of the distributive law, and once again (B.170) and Proposition B.169, one reduces to the case in which $J=G / H$ is a single orbit, and $X$ corresponds to $S^{k \rho_{H}}$ with $k|H| \geq n$ or $S^{k \rho_{H}-1}$, with $k|H|-1 \geq n$. In the first case

$$
X^{\wedge J} \approx S^{k \rho_{G}}
$$

has dimension $k|G| \geq k|H| \geq n$. In the second case

$$
X^{\wedge J} \approx S^{(n-1) \rho_{G}+V}
$$

where $V=\rho_{G}-\operatorname{ind}_{H}^{G} 1$. Write $W=\operatorname{ind}_{H}^{G}-1$ so that $S^{W} \wedge S^{V} \approx S^{\rho_{G}-1}$, and there is a cofibration sequence

$$
S(W)_{+} \wedge X^{\wedge J} \rightarrow X^{\wedge J} \rightarrow S^{(n-1) \rho_{G}+\left(\rho_{G}-1\right)} .
$$

The $G$-space $S(W)$ is homeomorphic to the boundary of the simplex with vertices $G / H$, and has no $G$-fixed points. The barycentric subdivision gives $S(W)_{+}$the structure of a $G$-CW complex built entirely from induced $G$-cells. It therefore follows from our induction hypothesis that

$$
S(W)_{+} \wedge X^{\wedge J}
$$

is slice $(n-1)$-positive. The rightmost term in (B.174) is a slice cell of dimension

$$
k|G|-1 \geq k|H|-1 \geq n
$$

It follows that $X^{\wedge J}$ is slice $(n-1)$-positive.

Remark B.175. We will later need to know that in the situation of Proposition B.171, one has

$$
\Sigma^{-1}(\Sigma X)^{\wedge J} \geq n .
$$

To see this, rewrite the spectrum in (B.176) as

$$
\left(\Sigma^{-1}\left(S^{1}\right)^{\wedge J}\right) \wedge\left(X^{\wedge J}\right) .
$$

The factor $\Sigma^{-1}\left(S^{1}\right)^{\wedge J}$ is weakly equivalent to the sphere $S^{V}$ with $V=\mathbb{R}^{J}-1$. This gives

$$
\Sigma^{-1}\left(S^{1}\right)^{\wedge J} \geq 0
$$

and the relation (B.176) then follows from Proposition B.171 and (B.170). 
We next turn to indexed symmetric powers. As in $\S$ B. 6 we consider a finite $G$-set $I$, a $G$-stable subgroup $\Sigma \subset \Sigma_{I}$, and the indexed symmetric power

$$
\operatorname{Sym}_{\Sigma}^{I} X=X^{\wedge I} / \Sigma \text {. }
$$

Proposition B.177. Let $n \geq 0$ be an integer, $I$ a non-empty $G$-set, and $X$ a cofibrant equivariant I-diagram. If $X$ is slice $(n-1)$-positive then both the indexed symmetric power $\operatorname{Sym}_{\Sigma}^{I} X$ and $\Sigma^{-1} \operatorname{Sym}_{\Sigma}^{I}(\Sigma X)$ are slice $(n-1)$-positive.

Proof: Using the equivalences

$$
\begin{gathered}
\left(E_{G} \Sigma\right)_{+} \hat{\Sigma} X^{\wedge I} \approx \operatorname{Sym}_{\Sigma}^{I} X \\
\Sigma^{-1}\left(E_{G} \Sigma\right)_{+} \hat{\Sigma}(\Sigma X)^{\wedge I} \approx \Sigma^{-1} \operatorname{Sym}_{\Sigma}^{I}(\Sigma X)
\end{gathered}
$$

of Proposition B.117 and working through an equivariant cell decomposition of $E_{G} \Sigma$ reduces the claim to showing that

$$
S_{+}{ }_{\Sigma} X^{\wedge I} \text { and } \Sigma^{-1} S_{+} \underset{\Sigma}{\wedge}(\Sigma X)^{\wedge I}
$$

are slice $(n-1)$-positive when $S$ is a finite $\Sigma$-free $\Sigma \rtimes G$-set. But the first spectrum in (B.178) is an indexed wedge of indexed smash products of $X$ (see the proof of Lemma B.115), hence slice $(n-1)$-positive by Propositions B.171 and B.169. The second spectrum is an indexed wedge of desuspensions of indexed smash products of $\Sigma X$, hence slice $(n-1)$-positive by Remark B.175 and Proposition B.169.

We can now investigate the slice sections of commutative rings. Let $P_{\text {alg }}^{n}$ : $\mathrm{Comm}^{G} \rightarrow \mathrm{Comm}^{G}$ be the multiplicative analogue of $P^{n}$, constructed as the colimit of a sequence of functors

$$
W_{0}^{\text {alg }} R \rightarrow W_{1}^{\text {alg }} R \rightarrow \cdots .
$$

The $W_{i}^{\text {alg }} R$ are defined inductively starting with $W_{0}^{\text {alg }} R=R$, and in which $W_{k}^{\text {alg }} R$ is defined by the pushout square

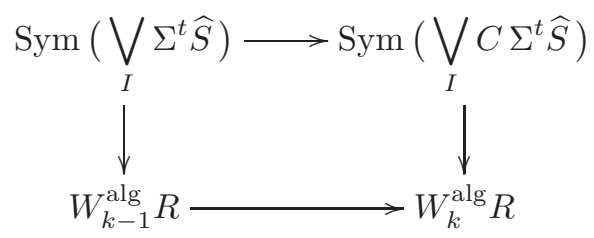

in which the indexing set $I$ is the set of maps $\Sigma^{t} \widehat{S} \rightarrow W_{k-1}^{\mathrm{alg}} R$ with $\widehat{S}>n$ a cofibrant slice cell and $t \geq 0$. The functor $P_{\text {alg }}^{n}$ is homotopical and for any $R$, the map $R \rightarrow P_{\text {alg }}^{n} R$ is a cofibration of equivariant commutative rings. The arrow $R \rightarrow P_{\text {alg }}^{n} R$ is characterized up to weak equivalence by the following universal property: if $S$ is an equivariant commutative ring whose underlying spectrum is slice $(n+1)$-null then the map

$$
\text { ho } \operatorname{Comm}^{G}\left(P_{\text {alg }}^{n} R, S\right) \rightarrow \text { ho } \operatorname{Comm}^{G}(R, S)
$$

is an isomorphism.

For clarity let's temporarily denote by $U$, the forgetful functor

$$
U: \mathbf{C o m m}^{G} \rightarrow \mathcal{S}^{G} \text {. }
$$


By the small object argument, the spectrum $U P_{\text {alg }}^{n} R$ is slice $(n+1)$-null, so there is a natural transformation

$$
P^{n} U R \rightarrow U P_{\mathrm{alg}}^{n} R
$$

of functors to $\mathcal{S}^{G}$.

Proposition B.179. If $R$ is a slice (-1)-positive cofibrant equivariant commutative ring, then for all $n \in \mathbb{Z}$, the map

$$
P^{n} U R \rightarrow U P_{a l g}^{n} R
$$

is a weak equivalence.

Proof: When $n$ is negative, $P^{n} U R$ is contractible, and $P_{\text {alg }}^{n} R$ is a commutative ring whose unit is nullhomotopic, hence also contractible. We may therefore assume $n$ is non-negative.

It suffices to show that each of the maps

$$
U W_{k-1}^{\mathrm{alg}} R \rightarrow U W_{k}^{\mathrm{alg}} R
$$

is a $P^{n}$-equivalence. We do this by working through the filtration used in the proof of Lemma B.132, whose successive terms are related by the homotopy cocartesian square

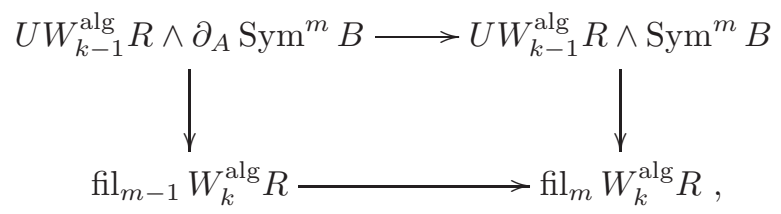

in which $A \rightarrow B$ is the map

$$
\bigvee_{I} \Sigma^{t} \widehat{S} \rightarrow \bigvee_{I} C \Sigma^{t} \widehat{S}
$$

By induction we may assume that the maps

$$
U R \rightarrow U W_{k-1}^{\mathrm{alg}} R \rightarrow \mathrm{fil}_{m-1} W_{k}^{\mathrm{alg}} R
$$

are $P^{n}$ equivalences, and so among other things that the three spectra are all in $\mathcal{S}_{\geq 0}^{G}$. The homotopy fiber of $\mathrm{fil}_{m-1} W_{k}^{\mathrm{alg}} R \rightarrow \mathrm{fil}_{m} W^{\text {alg }} R$ is $U W_{k-1}^{\text {alg }} R \wedge \Sigma^{-1} \operatorname{Sym}^{m}(B / A)$. Now $B / A$ is the suspension of the left term in (B.180) which is slice $n$-positive. It follows (Proposition B.177) that $\Sigma^{-1} \mathrm{Sym}^{m}(B / A)$ is also slice $n$-positive hence so is $U W_{k-1}^{\text {alg }} R \wedge \Sigma^{-1} \operatorname{Sym}^{m}(B / A)$ since $U W_{k-1}^{\text {alg }} R \geq 0$. The fact that fil $m-1 W_{k}^{\text {alg }} R \rightarrow$ fil $_{m} W^{\text {alg }} R$ is a $P^{n}$-equivalence is now a consequence of Lemma 4.28.

B.10. Geometric and monoidal geometric fixed points. The geometric fixed point functor was defined and its main properties summarized in §2.5.2. In this section we gives proofs of some of these properties, and describe the variation constructed in Mandell-May [53, §V.4]. We refer to the Mandell-May construction as the monoidal geometric fixed point functor and denote it $\Phi_{M}^{G}$, in order not to confuse it with the usual geometric fixed point functor. 
B.10.1. Geometric fixed points. The geometric fixed point functor was defined in $\S 2.5 .2$ by

$$
\Phi^{G}(X)=\left((\tilde{E} \mathcal{P} \wedge X)_{f}\right)^{G},
$$

in which the $G$-CW complex $\tilde{E} \mathcal{P}$ is the one characterized up to equivariant homotopy equivalence by the property

$$
(\tilde{E} \mathcal{P})^{H} \sim \begin{cases}S^{0} & H=G \\ * & H \neq G\end{cases}
$$

The characterizing property of $\tilde{E} \mathcal{P}$ implies that for any $G$-space $Z$ and any $G$-CW complex $A$, the restriction map

$$
[A, \tilde{E} \mathcal{P} \wedge Z]^{G} \rightarrow\left[A^{G}, \tilde{E} \mathcal{P} \wedge Z\right]^{G}
$$

is an isomorphism. Since $G$-acts trivially on $A^{G}$, the right hand side is isomorphic to

$$
\left[A^{G},(\tilde{E} \mathcal{P} \wedge Z)^{G}\right]=\left[A^{G}, Z^{G}\right] .
$$

Combining these gives the isomorphism

$$
[A, \tilde{E} \mathcal{P} \wedge Z]^{G} \approx\left[A^{G}, X^{G}\right] .
$$

This isomorphism is the foundation for our investigation into $\Phi^{G}$.

Let $\iota: \mathcal{S} \rightarrow \mathcal{S}^{G}$ be the functor which regards a spectrum as a $G$-spectrum with trivial action. As described in $\S 2.5 .1$, the fixed point functor $(-)^{G}$ is right adjoint to $\iota$

$$
\iota: \mathcal{S} \leftrightarrows \mathcal{S}^{G}:(-)^{G}
$$

and together they form a Quillen morphism in the positive complete model structures.

Since smashing with $\tilde{E} \mathcal{P}$ is homotopical, and the fixed point functor $(-)^{G}$ is a right Quillen functor, the functor $\Phi^{G}$ is homotopical.

Proposition B.182. For a spectrum $X \in \mathcal{S}$, the map

$$
X \rightarrow \Phi^{G}(\iota X)
$$

adjoint to

$$
\iota X \rightarrow \tilde{E} \mathcal{P} \wedge \iota X \rightarrow(\tilde{E} \mathcal{P} \wedge \iota X)_{f} .
$$

is a weak equivalence.

Proof: We have

$$
\begin{aligned}
\pi_{k} \Phi^{G}\left(i_{*} X\right) & \approx \operatorname{ho} \mathcal{S}\left(S^{k},(\tilde{E} \mathcal{P} \wedge X)_{f}^{G}\right) \\
& \approx \operatorname{ho} \mathcal{S}\left(S^{k},(\tilde{E} \mathcal{P} \wedge X)^{G}\right) \\
& \approx \operatorname{hos} \mathcal{S}^{G}\left(S^{k}, \tilde{E} \mathcal{P} \wedge X\right) \\
& \approx \lim _{W>-k} \pi_{k+W}^{G} \tilde{E} \mathcal{P} \wedge X_{W} \\
& \approx \underset{W>-k}{\lim _{W+W^{G}} X_{W^{G}}} \pi_{k}^{G}
\end{aligned}
$$


with the last isomorphism coming from (B.181). Under the composite isomorphism, the map on stable homotopy groups induced by (B.183) is

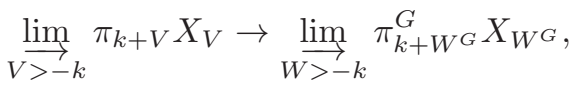

in which $V$ is ranging through the poset of finite dimensional orthogonal vector spaces and $W$ through the poset of $G$-representations. This is clearly an isomorphism.

Since $\tilde{E} \mathcal{P}$ is $H$-equivariantly contractible when $H$ is a proper subgroup of $G$, the smash product $\tilde{E} \mathcal{P} \wedge X$ is contractible if $X$ is a cellular spectrum built entirely from $G$-cells induced from a proper subgroup of $G$. More generally

Lemma B.184. Let $A$ and $Y$ be $G$-spectra. If $X$ is constructed from $A$ by attaching $G$-cells induced from proper subgroups, then the inclusion $A \rightarrow X$ induces a weak equivalence

$$
\tilde{E} \mathcal{P} \wedge A \wedge Y \stackrel{\sim}{\rightarrow} \tilde{E} \mathcal{P} \wedge X \wedge Y
$$

hence a weak equivalence

$$
\Phi^{G}(A \wedge Y) \stackrel{\sim}{\rightarrow} \Phi^{G}(X \wedge Y) .
$$

Corollary B.185. Let $V$ be a $G$-representation and $A$ a $G$-CW complex. The maps

$$
S^{-V^{G}} \wedge A^{G} \rightarrow S^{-V^{G}} \wedge A \leftarrow S^{-V} \wedge A
$$

induce weak equivalences

$$
S^{-V^{G}} \wedge A^{G} \sim \Phi^{G}\left(S^{-V^{G}} \wedge A^{G}\right) \stackrel{\sim}{\rightarrow} \Phi^{G}\left(S^{-V^{G}} \wedge A\right) \stackrel{\sim}{\leftarrow} \Phi^{G}\left(S^{-V} \wedge A\right)
$$

giving a zig-zag of weak equivalences

$$
\Phi^{G}\left(S^{-V} \wedge A\right) \stackrel{\sim}{\leftrightarrow} S^{-V^{G}} \wedge A^{G} .
$$

Proof: We work our way from the left. The weak equivalence $S^{-V^{G}} \wedge A^{G} \approx$ $\Phi^{G}\left(A^{G} \wedge S^{-V^{G}}\right)$ is Proposition B.182. The next map is a weak equivalence by Lemma B.184 since $A$ is constructed from $A^{G}$ by adding induced $G$-cells. The last map can be constructed by applying $\Phi^{G}$ to the composition

$$
S^{-V} \wedge A \rightarrow S^{-V} \wedge S^{V-V^{G}} \wedge A \rightarrow S^{-V^{G}} \wedge A .
$$

The right arrow is a weak equivalence. Since $S^{V-V^{G}}$ is a $G$-CW complex with fixed point space $S^{0}$, it is constructed from $S^{0}$ by adding induced $G$-cells. The left map therefore induces an equivalence of geometric fixed points by Lemma B.184.

B.10.2. Motivation and definition of the monoidal geometric fixed point functor. For an orthogonal representation $V$ of $G$ let $V^{G} \subset V$ be the space of invariant vectors, and $V^{\perp}$ the orthogonal complement of $V^{G}$. Note that

$$
\mathscr{J}_{G}(V, W)^{G} \approx \mathscr{J}\left(V^{G}, W^{G}\right) \wedge O\left(V^{\perp}, W^{\perp}\right)_{+}^{G},
$$

so that there is a canonical map

$$
\mathscr{J}_{G}(V, W)^{G} \rightarrow \mathscr{J}\left(V^{G}, W^{G}\right),
$$

given in terms of (B.186) by smashing the identity map with the map $O\left(V^{\perp}, W^{\perp}\right)^{G} \rightarrow$ pt. 
We wish to define a functor $\Phi_{M}^{G}$ with the property that

$$
\Phi_{M}^{G}\left(S^{-V} \wedge A\right)=S^{-V^{G}} \wedge A^{G}
$$

and which commutes with colimits as far as is possible. A value needs to be assigned to the effect of $\Phi_{M}^{G}$ on the map

$$
S^{-W} \wedge \mathscr{J}_{G}(V, W) \rightarrow S^{-V}
$$

The only obvious choice is to take

$$
\Phi_{M}^{G}\left(S^{-W} \wedge \mathscr{J}_{G}(V, W)\right) \rightarrow \Phi_{M}^{G}\left(S^{-V}\right)
$$

to be the composite

$$
S^{-W^{G}} \wedge \mathscr{J}_{G}(V, W)^{G} \rightarrow S^{-W^{G}} \wedge \mathscr{J}\left(V^{G}, W^{G}\right) \rightarrow S^{-V^{G}} .
$$

If $\Phi_{M}^{G}$ actually were to commute with colimits, it would be determined by the specifications given by (B.187) and (B.188). Indeed, using the tautological presentation to write a general equivariant orthogonal spectrum $X$ as a reflexive coequalizer

$$
\bigvee_{V, W} S^{-W} \wedge \mathscr{J}_{G}(V, W) \wedge X_{V} \rightrightarrows \bigvee_{V} S^{-V} \wedge X_{V} \rightarrow X
$$

the value of $\Phi_{M}^{G}(X)$ would be given by the reflexive coequalizer diagram

$$
\bigvee_{V, W} S^{-W^{G}} \wedge \mathscr{J}_{G}(V, W)_{+}^{G} \wedge X_{V}^{G} \rightrightarrows \bigvee_{V} S^{-V^{G}} \wedge X_{V}^{G} \rightarrow \Phi_{M}^{G} X
$$

We take this as the definition of $\Phi_{M}^{G}(X)$.

Definition B.190. The monoidal geometric fixed point functor

$$
\Phi_{M}^{G}: \mathcal{S}^{G} \rightarrow \mathcal{S}
$$

is the functor defined by the coequalizer diagram (B.189).

Remark B.191. In case $X=S^{-V} \wedge A$, the tautological presentation is a split coequalizer, and one recovers both (B.187) and (B.188).

A fundamental property of the usual geometric fixed point functor $\Phi^{G}$ is that for proper $H \subset G$, the spectrum $\Phi^{G}\left(G_{+}{ }_{H} X\right)$ is contractible. The monoidal geometric fixed point functor has this property on the nose.

Proposition B.192. Suppose that $J$ is a $G$-set and $X$ an equivariant $J$-diagram. If $J$ has no $G$-fixed points then the map

$$
\Phi_{M}^{G}\left(\bigvee_{j \in J} X_{j}\right) \rightarrow *
$$

is an isomorphism. In particular, if $H \subset G$ is a proper subgroup and $X$ an orthogonal $H$-spectrum, then the map

$$
\Phi_{M}^{G}\left(G_{+} \stackrel{\wedge}{H}^{\prime} X\right) \rightarrow *
$$

is an isomorphism. 
Proof: Since indexed wedges are computed componentwise, the assumption that $J$ has no fixed points implies that for all representations $W$ of $G$,

$$
\left(\bigvee_{j \in J} X_{j}\right)_{W}^{G}=\left(\bigvee_{j \in J}\left(X_{j}\right)_{W}\right)^{G}=*
$$

The claim then follows from the definition of $\Phi_{M}^{G}$.

Working through an equivariant cell decomposition gives

Corollary B.193. Let $A$ and $Y$ be $G$-spectra. If $X$ is constructed from $A$ by attaching $G$-cells induced from proper subgroups, then the map $\Phi_{M}^{G}(A \wedge Y) \rightarrow \Phi_{M}^{G}(X \wedge Y)$ is an isomorphism.

There is a natural map

$$
X^{G} \rightarrow \Phi_{M}^{G} X
$$

from the fixed point spectrum of $X$ to the monoidal geometric fixed point spectrum. To construct it note that the fixed point spectrum of $X$ is computed termwise, and so is given by the coequalizer diagram

$$
\bigvee_{V, W \in \mathscr{J}} S^{-W} \wedge \mathscr{J}(V, W)_{+} \wedge X_{V}^{G} \rightrightarrows \bigvee_{V \in \mathscr{J}} S^{-V} \wedge X_{V}^{G} \rightarrow X^{G}
$$

The map (B.194) is given by the evident inclusion of (B.195) into (B.189).

The functor $\Phi_{M}^{G}$ cannot commute with all colimits. However, since colimits of orthogonal $G$-spectra are computed objectwise, the definition implies that $\Phi_{M}^{G}$ commutes with whatever enriched colimits are preserved by the fixed point functor on $G$-spaces. This means that there is a functorial isomorphism

$$
\Phi_{M}^{G}(X \wedge A) \approx \Phi_{M}^{G}(X) \wedge A^{G}
$$

for each pointed $G$-space $A$, and that $\Phi_{M}^{G}$ commutes with the formation of wedges, directed colimits and cobase change along a closed inclusion. Because $h$-cofibrations and cofibrations are objectwise closed inclusion (Lemma A.70 and Remark 5.38), the functor $\Phi_{M}^{G}$ has good homotopy theoretic properties.

B.10.3. Homotopy properties of $\Phi_{M}^{G}$. Several variations on the following appear in in $[53, \S \mathrm{V} .4]$.

Proposition B.197. The functor $\Phi_{M}^{G}$ sends cofibrations to cofibrations and acyclic cofibrations to acyclic cofibrations. It therefore sends weak equivalences between cofibrant objects to weak equivalences.

Proof: That $\Phi_{M}^{G}$ sends cofibrations to cofibrations follows from the fact that it preserves cobase change along closed inclusions and sends generating cofibrations to generating cofibrations. A similar argument applies to the acyclic cofibrations, once one checks that $\Phi_{M}^{G}$ sends both maps in the factorization (B.67)

$$
S^{-V \oplus W} \wedge S^{W} \rightarrow \tilde{S}^{-V} \rightarrow S^{-V}
$$

to weak equivalences. But the second map is a homotopy equivalence and the composite map is sent to a weak equivalence by (B.187). The last assertion is a consequence of Ken Brown's Lemma.

Proposition B.197 implies that the monoidal geometric fixed point functor has a left derived functor which can be computed on any cofibrant approximation. A 
similar argument with a slightly different model structure could be used to show that the left derived functor can be computed on a cellular approximation. We will show in $\S$ B.10.5 that the left derived functor $\mathbf{L} \Phi_{M}^{G}$ is the geometric fixed point functor $\Phi^{G}$.

B.10.4. Monoidal geometric fixed points and smash product. The properties (B.187) and (B.188) give an identification

$$
\Phi_{M}^{G}\left(S^{-V} \wedge A \wedge S^{-W} \wedge B\right) \approx \Phi_{M}^{G}\left(S^{-V} \wedge A\right) \wedge \Phi_{M}^{G}\left(S^{-W} \wedge B\right)
$$

making the diagram

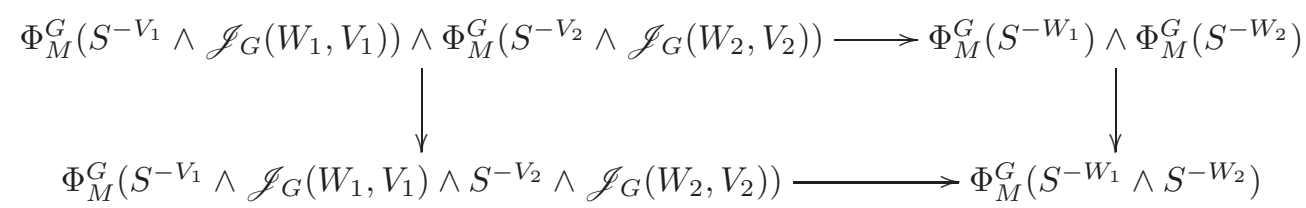

commute. Applying $\Phi_{M}^{G}$ termwise to the smash product of the tautological presentations of $X$ and $Y$, and using the above identifications, gives a natural transformation

$$
\Phi_{M}^{G}(X) \wedge \Phi_{M}^{G}(Y) \rightarrow \Phi_{M}^{G}(X \wedge Y),
$$

making $\Phi_{M}^{G}$ lax monoidal. From the formula (B.187) this map is an isomorphism if $X=S^{-V} \wedge A$ and $Y=S^{-W} \wedge B$. This leads to

Proposition B.199 ([53], Proposition V.4.7). The functor $\Phi_{M}^{G}$ is weakly monoidal: the map (B.198) is a weak equivalence (in fact an isomorphism) if $X$ and $Y$ are cellular.

Proof: The class of spectra $X$ and $Y$ for which (B.198) is an isomorphism is stable under smashing with a $G$-space, the formation of wedges, directed colimits, and cobase change along an objectwise closed inclusion. Since (B.198) is an isomorphism when $X=G_{+}{ }_{H} S^{-V} \wedge A$ and $Y=G_{+}{ }_{H}^{\wedge} S^{-W} \wedge B$ this implies it is an isomorphism when $X$ and $Y$ are cellular. Since isomorphisms are weak equivalences, the result follows.

Remark B.200. Blumberg and Mandell [10, Appendix A] have shown that Proposition B.199 remains true under the assumption that only one of $X$ or $Y$ is cellular. This implies that Proposition B.203 below remains true if only one of $N$ or $N^{\prime}$ is cofibrant.

B.10.5. Relation with the geometric fixed point functor. We now turn to identifying the left derived functor $\mathbf{L} \Phi_{M}^{G}$ with the geometric fixed point functor $\Phi^{G}$. The inclusion $S^{0} \rightarrow \tilde{E} \mathcal{P}$ and the fibrant replacement functor give maps

$$
X \rightarrow \tilde{E} \mathcal{P} \wedge X \rightarrow(\tilde{E} \mathcal{P} \wedge X)_{f} .
$$

Proposition B.201 ([53], Proposition V.4.17). If $X$ is cofibrant, then the maps

$$
\Phi^{G} X=\left(\tilde{E} \mathcal{P} \wedge X_{f}\right)^{G} \rightarrow \Phi_{M}^{G}\left((\tilde{E} \mathcal{P} \wedge X)_{f}\right) \leftarrow \Phi_{M}^{G}(X)
$$

are weak equivalences. 
Sketch of proof: For the arrow on the left, note that both functors are homotopical and, up to weak equivalence, preserve filtered colimits along $h$-cofibrations. Using the canonical homotopy presentation, it suffices to check that the arrow on the left is a weak equivalence when $X=S^{-V} \wedge A$, with $A$ a $G$-CW complex. This follows from Corollary B.185, the identity (B.187), and a little diagram chasing to check compatibility.

The right arrow is the composition of

$$
\Phi_{M}^{G}(X) \rightarrow \Phi_{M}^{G}(\tilde{E} \mathcal{P} \wedge X)
$$

which is an isomorphism by (B.196), and

$$
\Phi_{M}^{G}(\tilde{E} \mathcal{P} \wedge X) \rightarrow \Phi_{M}^{G}\left((\tilde{E} \mathcal{P} \wedge X)_{f}\right),
$$

which is an acyclic cofibration by Proposition B.197.

B.10.6. The relative monoidal geometric fixed point functor. The functor $\Phi_{M}^{G}$ can be formulated relative to an equivariant commutative or associative algebra $R$. As described below, care must be taken in using the theory in this way.

Because it is lax monoidal, the functor $\Phi_{M}^{G}$ gives a functor

$$
\Phi_{M}^{G}: \mathcal{M}_{R} \rightarrow \mathcal{M}_{\Phi_{M}^{G} R}
$$

which is lax monoidal in case $R$ is commutative.

Proposition B.202. The functor

$$
\Phi_{M}^{G}: \mathcal{M}_{R} \rightarrow \mathcal{M}_{\Phi_{M}^{G} R}
$$

commutes with cobase change along a cofibration and preserves the classes of cofibrations and acyclic cofibrations.

Proof: This follows easily from the fact that the maps of equivariant orthogonal spectra underlying the generating cofibrations for $\mathcal{M}_{R}$ are $h$-cofibrations.

Proposition B.203. When $R$ is commutative, the functor

$$
\Phi_{M}^{G}: \mathcal{M}_{R} \rightarrow \mathcal{M}_{\Phi_{M}^{G} R}
$$

is weakly monoidal, and in fact

$$
\Phi_{M}^{G}\left(N^{\prime}\right) \underset{\Phi_{M}^{G}(R)}{\wedge} \Phi_{M}^{G}(N) \rightarrow \Phi_{M}^{G}\left(N^{\prime} \underset{R}{\wedge} N\right)
$$

is an isomorphism if $N^{\prime}$ and $N$ are cofibrant.

Proof: The proof is the same as that of Proposition B.199 once one knows that the class of modules $N^{\prime}$ and $N$ for which (B.204) is an isomorphism is stable under cobase change along a generating cofibration. This, in turn, is a consequence of the fact that both sides of (B.204) preserve $h$-cofibrations in each variable, since $h$-cofibrations are closed inclusions. The functor $\Phi_{M}^{G}$ does so since it commutes with the formation of mapping cylinders, and $N^{\prime}{ }_{R}^{\wedge}(-)$ does since $\mathcal{M}_{R}$ is a closed symmetric monoidal category.

As promising as it looks, it is not so easy to make use of Proposition B.203. The trouble is that unless $X$ is cofibrant, $\Phi_{M}^{G}(X)$ may not have the weak homotopy type of $\Phi^{G}(X)$. So in order to use Proposition B.203 one needs a condition guaranteeing 
that $N^{\prime} \stackrel{\wedge}{\wedge} N$ is a cofibrant spectrum. The criterion of Proposition B.205 below was suggested to us by Mike Mandell.

Proposition B.205. Suppose $R$ is an associative algebra with the property that $S^{-1} \wedge R$ is cofibrant. If $N^{\prime}$ is a cofibrant right $R$-module, and $S^{-1} \wedge N$ is a cofibrant left $R$-module, then

$$
N^{\prime} \underset{R}{\wedge} N
$$

is cofibrant.

Proof: First note that the condition on $R$ guarantees that for every representation $U$ with $\operatorname{dim} U^{G}>0$ and every cofibrant $G$-space $T$, the spectrum

$$
S^{-U} \wedge R \wedge T
$$

is cofibrant. Since the formation of $N^{\prime}{ }_{R} N$ commutes with cobase change in both variables, the result reduces to the case $N^{\prime}=S^{-V} \wedge R \wedge X$ and $N=S^{-W} \wedge R \wedge Y$ with $V$ having a non-zero fixed point space, and $X$ and $Y$ cofibrant $G$-spaces. But in that case

$$
N^{\prime} \underset{R}{\wedge} N \approx S^{-V \oplus W} \wedge R \wedge X \wedge Y
$$

which is of the form (B.206), and hence cofibrant.

Corollary B.207. Suppose $R$ is an associative algebra with the property that $S^{-1} \wedge$ $R$ is cofibrant. If $N^{\prime}$ is a cofibrant right $R$-module, then the equivariant orthogonal spectrum underlying $N^{\prime}$ is cofibrant.

Proof: Just take $N=R$ in Proposition B.205.

The following result plays an important role in determining $\Phi^{G} R(\infty)$ (§7.3).

Proposition B.208. Suppose that $R$ is an equivariant associative algebra whose underlying $G$-spectrum is cellular, and that $R \rightarrow S^{0}$ is an equivariant associative algebra map. If $N^{\prime}$ is a cofibrant right $R$-module, then $N^{\prime}{ }_{R}^{\wedge} S^{0}$ is a cofibrant spectrum, and the map

$$
\Phi_{M}^{G}\left(N^{\prime}\right) \underset{\Phi_{M}^{G} R}{\wedge} S^{0} \rightarrow \Phi_{M}^{G}\left(N^{\prime} \underset{R}{\wedge} S^{0}\right)
$$

is an isomorphism.

Proof: One easily reduces to the case $N^{\prime}=S^{-V} \wedge X \wedge R$, in which $V$ is a representation with $V^{G} \neq 0$, and $X$ is a cofibrant $G$-space. In this case $N^{\prime} \wedge_{R} S^{0}$ is isomorphic to $S^{-V} \wedge X$ which is cofibrant. The assertion about monoidal geometric fixed points follows easily from Proposition B.199. 
B.11. Geometric fixed points and the norm. Our original version of the following result merely concluded that the transformation in question was a weak equivalence on cofibrant objects. Andrew Blumberg and Mike Mandell pointed out that it is in fact an isomorphism. At their request we have included the stronger statement.

Proposition B.209. Suppose $H \subset G$. There is a natural transformation

$$
\Phi_{M}^{H}(-) \rightarrow \Phi_{M}^{G} \circ N_{H}^{G}(-)
$$

which is an isomorphism, hence a weak equivalence on cellular objects.

Proof: To construct the natural transformation, first note that there is a natural isomorphism

$$
A^{H} \approx\left(N_{H}^{G} A\right)^{G}
$$

for $H$-equivariant spaces $A$. Next note that for an orthogonal representation $V$ of $H$, Proposition A.59 and the property (B.187) give isomorphisms

$$
\Phi_{M}^{G} N_{H}^{G} S^{-V} \approx \Phi_{M}^{G} S^{-\operatorname{ind}_{H}^{G} V} \approx S^{-V^{H}} \approx \Phi^{H} S^{-V} .
$$

The monoidal properties of $\Phi_{M}^{G}$ and the norm then combine to give an isomorphism

$$
\Phi^{H}\left(S^{-V} \wedge A\right) \approx \Phi^{G} N_{H}^{G}\left(S^{-V} \wedge A\right)
$$

which one easily checks to be compatible with the maps

$$
S^{-V} \wedge \mathscr{J}_{H}(W, V) \rightarrow S^{-W}
$$

To construct the transformation, write a general $H$-spectrum $X$ in terms of its tautological presentation

$$
\bigvee_{V, W} S^{-W} \wedge \mathscr{J}_{H}(V, W) \wedge X_{V} \rightrightarrows \bigvee_{V} S^{-V} \wedge X_{V} \rightarrow X,
$$

and apply (B.210) termwise to produce a diagram

$$
\bigvee_{V, W} S^{-W^{H}} \wedge \mathscr{J}_{H}(V, W)^{H} \wedge X_{V}^{H} \rightrightarrows \bigvee_{V} S^{-V^{H}} \wedge X_{V}^{H} \rightarrow \Phi^{G} N_{H}^{G} X
$$

The coequalizer of the two arrows is, by definition, $\Phi_{M}^{H}(X)$. This gives the natural transformation.

The isomorphism assertion for cellular $X$ reduces to the special case (B.210), once one show that $\Phi_{M}^{G} \circ N_{H}^{G}(-)$ commute with the formation of wedges, cobase change along cofibrations between cofibrant objects, and filtered colimits along closed inclusions. The last property is clear since both of the functors being composed commutes with filtered colimits along closed inclusions. For the other two assertions it will be easier to work in terms of equivariant $J$-diagrams for $J=G / H$.

Suppose that $T$ is an indexing set, and $X_{t}, t \in T$ a set of equivariant $J$-diagrams. We wish to show that the natural map

$$
\bigvee_{t \in T} \Phi_{M}^{G} X_{t}^{\wedge J} \rightarrow \Phi_{M}^{G}\left(\bigvee_{t \in T} X_{t}\right)^{\wedge J}
$$

is an isomorphism. For this use the distributive law to rewrite the argument of the right hand side as

$$
\bigvee_{\gamma \in \Gamma} X^{\wedge \gamma}
$$


where $\gamma$ is the $G$-set of functions $J \rightarrow T$ and

$$
X^{\wedge \gamma}=\bigwedge_{j \in J} X_{\gamma(j)} .
$$

The map asserted to be an isomorphism on monoidal geometric fixed points is the inclusion of the summand indexed by the constant functions. But since $G$ acts trivially on $T$, the other summands form an indexed wedge over a $G$-set with no fixed points. The claim then follows from Proposition B.192.

The cobase change property is similar. Suppose we are given a pushout square of equivariant $J$-diagrams

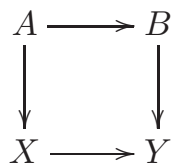

in which $A \rightarrow B$ is a cofibration and $A$ is cofibrant. We consider the filtration of $Y^{\wedge J}$ given in $\S$ A.3.4 whose stages fit into a pushout square

$$
\begin{array}{cc}
\bigvee_{\substack{J=J_{0} \amalg J_{1} \\
\left|J_{1}\right|=m}} X^{\wedge J_{0}} \wedge \partial_{A} B^{\wedge J_{1}} \longrightarrow & \bigvee_{\substack{J=J_{0} \amalg J_{1} \\
\left|J_{1}\right|=m}} X^{\wedge J_{0}} \wedge B^{\wedge J_{1}} \\
\operatorname{fil}_{m-1} Y^{\wedge J} & \downarrow \text { fil }_{m} Y^{\wedge J} .
\end{array}
$$

By Proposition B.97, the upper arrow is an $h$-cofibration, so the resulting diagram of monoidal geometric fixed points is a pushout. But since $J$ is a transitive $G$-set, unless $m=|J|$ the group $G$ has no fixed points on the $G$-set indexing the wedges. Applying Proposition B.192 then shows that for $m<|J|$ the map

$$
\Phi_{M}^{G} X^{\wedge J} \rightarrow \Phi_{M}^{G} \mathrm{fil}_{m} Y^{\wedge J}
$$

is an isomorphism, and that the pushout square when $m=|J|$ becomes

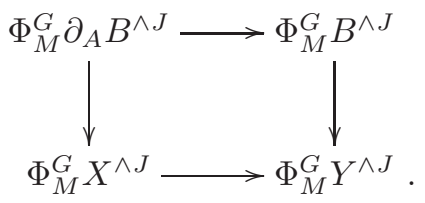

However the term $\partial_{A} B^{\wedge J}$ is the term fil $J_{\mid-1} B^{\wedge J}$ in the case in which $X=A$ and $Y=B$, and so $\Phi_{M}^{G} A^{\wedge J} \rightarrow \Phi_{M}^{G} \partial_{A} B^{\wedge J}$ is an isomorphism. This completes the proof.

Thinking in terms of left derived functors one can get a slightly better result. As long as $X$ has the property that the map $\left(\mathbf{L} N_{H}^{G}\right) X \rightarrow N_{H}^{G} X$ is a weak equivalence, there will be a weak equivalence between $\Phi^{H} X$ and $\Phi^{G} N_{H}^{G} X$. Since it plays an important role in our work, we spell it out. Start with $X \in \mathcal{S}^{H}$ and let $X_{c} \rightarrow X$ be a cofibrant approximation. Now consider the diagram

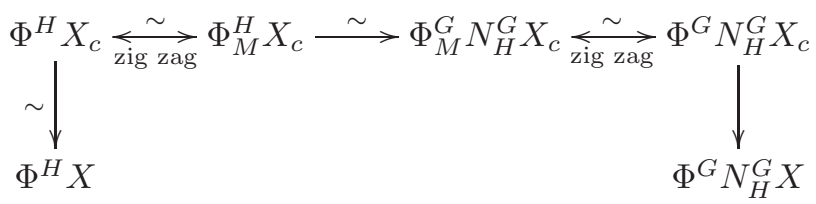


The left vertical arrows are weak equivalences since the geometric fixed point functor preserves weak equivalences. The weak equivalences in the top row are given by Propositions B.201, B.89, and B.209. Since $\Phi^{G}$ is homotopical we have

Proposition B.213. Suppose that $X \in \mathcal{S}^{H}$ has the property that for some (hence any) cofibrant approximation $X_{c} \rightarrow X$ the map

$$
N_{H}^{G} X_{c} \rightarrow N_{H}^{G} X
$$

is a weak equivalence. Then the functorial relationship between $\Phi^{H} X$ and $\Phi^{G} N_{H}^{G} X$ given by (B.212) is a weak equivalence.

Remark B.214. Proposition B.213 can be proved without reference to $\Phi_{M}^{G}$ by using the canonical homotopy presentation.

Remark B.215. Proposition B.213 applies in particular when $X$ is very flat in the sense of $\S$ B.8.2. By Proposition B.147 this means that if $R \in \mathcal{S}^{H}$ is a cofibrant commutative ring, then $\Phi^{H} R$ and $\Phi^{G} N_{H}^{G} R$ are related by a functorial zig-zag of weak equivalences. The case of interest to us is when $H=C_{2}, G=C_{2^{n}}$ and $R=M U_{\mathbb{R}}$. In this case $N_{H}^{G} R=M U^{((G))}$, and we get an equivalence

$$
\Phi^{G} M U^{((G))} \approx \Phi^{C_{2}} M U_{\mathbb{R}} \approx M O .
$$

Remark B.216. Proposition B.213 also applies to the suspension spectra of cofibrant $H$-spaces. Indeed, if $X$ is a cofibrant $H$-space then $S^{-1} \wedge S^{1} \wedge X \rightarrow X$ is a cofibrant approximation. Applying $N_{H}^{G}$ leads to the map

$$
S^{-V} \wedge S^{V} \wedge N_{H}^{G}(X) \rightarrow N_{H}^{G}(X)
$$

with $V=\operatorname{ind}_{H}^{G} \mathbb{R}$, which is a weak equivalence (in fact a cofibrant approximation). This case is used to show that $\Phi^{G} \circ N_{H}^{G}$ is a ring homomorphism on the $R O(G)$ graded cohomology of $G$-spaces (Proposition 2.59).

B.12. Real bordism. In this section we give a construction of the real bordism spectrum $M U_{\mathbb{R}}$ as a commutative algebra in $\mathcal{S}^{C_{2}}$. As will be apparent to the reader, this construction owes a great deal to the Stefan Schwede's construction of $M U$ in [74, Chapter 2]. We are indebted to Schwede for some very helpful correspondence concerning these matters.

Our goal is to construct a $C_{2}$-equivariant commutative ring $M U_{\mathbb{R}}$ admitting the canonical homotopy presentation

$$
M U_{\mathbb{R}} \approx \operatorname{holim} S^{-\mathbb{C}^{n}} \wedge M U(n),
$$

in which $M U(n)$ is the Thom complex of the universal bundle over $B U(n)$. The group $C_{2}$ is acting on everything by complex conjugation, so we could also write this expression as

$$
M U_{\mathbb{R}} \approx \operatorname{holim} S^{-n \rho_{2}} \wedge M U(n) .
$$

The map

$$
S^{-\rho_{2}} \wedge M U(1) \rightarrow M U_{\mathbb{R}}
$$

defines a real orientation. These things form the basis for everything we proved about $M U_{\mathbb{R}}$.

The most natural construction of $M U_{\mathbb{R}}$ realizes this structure in the category $\mathcal{S}_{\mathbb{R}}$ of real spectra, which is related to the category of $C_{2}$-equivariant orthogonal spectra by a multiplicative Quillen equivalence

$$
i_{!}: \mathcal{S}_{\mathbb{R}} \leftrightarrows \mathcal{S}^{C_{2}}: i^{*}
$$


We will construct a commutative algebra $\mathcal{M} \mathcal{U}_{\mathbb{R}} \in \operatorname{comm} \mathcal{S}_{\mathbb{R}}$, whose underlying real spectrum has a canonical homotopy presentation of the form

$$
\mathcal{M} \mathcal{U}_{\mathbb{R}} \stackrel{\sim}{\sim} \operatorname{holim}_{\longrightarrow} S^{-n \mathbb{C}} \wedge M U(n) \stackrel{\sim}{\rightarrow} \operatorname{holim}_{\longrightarrow} S^{-n \mathbb{C}} \wedge M U(n)_{\mathrm{cf}} .
$$

Applying $i_{\text {! }}$ to (B.219) and making the identification $i_{!} S^{-\mathbb{C}}=S^{-\rho_{2}}$ leads to the diagram

$$
i_{!} \mathcal{M U}_{\mathbb{R}} \leftarrow \operatorname{holim} S^{-n \rho_{2}} \wedge M U(n) \rightarrow \operatorname{holim} S^{-n \rho_{2}} \wedge M U(n)_{\mathrm{cf}}
$$

We define $M U_{\mathbb{R}}$ to be the spectrum $i_{!} \mathcal{M U}_{\mathbb{R}}^{\prime}$, where $\mathcal{M} \mathcal{U}_{\mathbb{R}}^{\prime} \rightarrow \mathcal{M} \mathcal{U}_{\mathbb{R}}$ is a cofibrant commutative algebra approximation. The functor $i_{\text {! }}$ is strictly monoidal, so $M U_{\mathbb{R}}$ is a commutative ring in $\mathcal{S}^{C_{2}}$. The map on the right in (B.220) is a weak equivalence since $i_{\text {! }}$ is a left Quillen functor. The problem is to show that the one on the left is.

This involves two steps. The first is to show that the forgetful functor

$$
\operatorname{comm} \mathcal{S}_{\mathbb{R}} \rightarrow \mathcal{S}_{\mathbb{R}}
$$

creates a model category structure on $\operatorname{comm} \mathcal{S}_{\mathbb{R}}$. This involves analyzing the symmetric powers of cofibrant real spectra, which, as pointed out in Remark B.120 depends in an essential way on understanding the homotopy theoretic properties of indexed symmetric powers. The second is to show that the functor $i_{\text {! }}$ is homotopical on a subcategory of $\mathcal{S}_{\mathbb{R}}$ containing the real spectra underlying cofibrant real commutative rings. As in our analysis of norms of commutative rings, this involves a generalized notion of flatness. There is no real way to short circuit the model structure on $\mathbf{c o m m} \mathcal{S}_{\mathbb{R}}$. Its role is to identify the cofibrant real commutative algebras. But the only real work in establishing the model structure is showing that what one thinks is a cofibrant approximation is actually a weak equivalence, and that is what is needed to show that every real commutative algebra is weakly equivalent to a cofibrant one.

B.12.1. Real and complex spectra. In this section we describe the basics of real and complex spectra. The additive results are more or less all a special case of the results of [54], but the important multiplicative properties require a separate analysis.

For finite dimensional complex Hermitian vector spaces $A$ and $B$ let $U(A, B)$ be the Stiefel manifold of unitary embeddings $A \hookrightarrow B$. There is a natural Hermitian inner product on the complexification $V_{\mathbb{C}}$ of a real orthogonal vector space $V$, so there is a natural map

$$
O(V, W) \rightarrow U\left(V_{\mathbb{C}}, W_{\mathbb{C}}\right)
$$

The group $C_{2}$ acts on $U\left(V_{\mathbb{C}}, W_{\mathbb{C}}\right)$ by complex conjugation, and the fixed point space is $O(V, W)$.

Definition B.221. The category $\mathscr{J}_{\mathbb{C}}$ is the topological category whose objects are finite dimensional Hermitian vector spaces, and whose morphism space $\mathscr{J}_{\mathbb{C}}(A, B)$ is the Thom complex

$$
\mathscr{J}_{\mathbb{C}}(A, B)=\operatorname{Thom}(U(A, B) ; B-A) .
$$

The category $\mathscr{J}_{\mathbb{R}}$ is the $C_{2}$-equivariant topological category whose objects are finite dimensional orthogonal real vector spaces $V$, and with

$$
\mathscr{J}_{\mathbb{R}}(V, W)=\mathscr{J}_{\mathbb{C}}\left(V_{\mathbb{C}}, W_{\mathbb{C}}\right)
$$

on which $C_{2}$ acts by complex conjugation. 
Definition B.222. The category $\mathcal{S}_{\mathbb{C}}$ of complex spectra is the topological category of (continuous) functors

$$
\mathscr{J}_{\mathbb{C}} \rightarrow \mathcal{T}
$$

The category $\mathcal{S}_{\mathbb{R}}$ of real spectra is the topological category of $C_{2}$-enriched functors

$$
\mathscr{J}_{\mathbb{R}} \rightarrow \underline{\mathcal{I}}_{C_{2}}
$$

and equivariant natural transformations.

We will write

$$
V \mapsto X_{V_{\mathbb{C}}}
$$

for a typical real spectrum $X$, and let $S^{-V_{\mathbb{C}}} \in \mathcal{S}_{\mathbb{R}}$ be the functor co-represented by $V \in \mathscr{J}_{\mathbb{R}}$. From the Yoneda lemma there is a natural isomorphism

$$
\mathcal{S}_{\mathbb{R}}\left(S^{-V_{\mathbb{C}}}, X\right)=X_{V_{\mathbb{C}}} .
$$

As with equivariant orthogonal spectra, every real spectrum $X$ has a tautological presentation

$$
\bigvee_{V, W \in \mathscr{J}_{\mathbb{R}}} S^{-W_{\mathbb{C}}} \wedge \mathscr{J}_{\mathbb{R}}(V, W) \wedge X_{W_{\mathbb{C}}} \rightrightarrows \bigvee_{V \in \mathscr{J}_{\mathbb{R}}} S^{-V_{\mathbb{C}}} \wedge X_{V_{\mathbb{C}}} \rightarrow X
$$

A similar apparatus exist for complex spectra.

Remark B.224. The category $\mathscr{J}_{\mathbb{R}}$ is equivalent to its full subcategory with objects $\mathbb{R}^{n}$, and similarly $\mathscr{J}_{\mathbb{C}}$ is equivalent to its full subcategory with objects $\mathbb{C}^{n}$. Thus a real spectrum $X$ is specified by the spaces $X_{V_{\mathbb{C}}}$ with $V=\mathbb{R}^{n}$ together with the structure maps between them, and an object $Y \in \mathcal{S}_{\mathbb{C}}$ is specified by its spaces $Y_{\mathbb{C}^{n}}$, together with the structure maps between them.

The group $C_{2}$ acts on $\mathcal{S}_{\mathbb{C}}$ through its action on $\mathscr{J}_{\mathbb{C}}$. We write this as $X \mapsto \bar{X}$, where

$$
(\bar{X})_{V}=X_{\bar{V}}
$$

A fixed point for this action is a complex spectrum $X$ equipped with an isomorphism $X \rightarrow \bar{X}$ having the property that $X \rightarrow \bar{X} \rightarrow \overline{\bar{X}}=X$ is the identity map. Restricting to the spaces $X_{\mathbb{C}^{n}}$ and using the standard basis to identify $\mathbb{C}^{n}$ with $\overline{\mathbb{C}}^{n}$ one sees that a fixed point for this $C_{2}$-action consists of a sequence $C_{2}$-spaces $X_{\mathbb{C}^{n}}$, together with an associative family $C_{2}$-equivariant maps

$$
\mathscr{J}_{\mathbb{C}}\left(\mathbb{C}^{n}, \mathbb{C}^{m}\right) \wedge_{U\left(\mathbb{C}^{n}\right)} X_{\mathbb{C}^{n}} \rightarrow X_{\mathbb{C}^{m}},
$$

where $C_{2}$ is acting by conjugation. But this is the same thing as giving a real spectrum indexed on the spaces $\mathbb{R}^{n}$. This shows that the category of fixed points for the $C_{2}$-action on $\mathcal{S}_{\mathbb{C}}$ is $\mathcal{S}_{\mathbb{R}}$.

B.12.2. Smash product and indexed smash products. The orthogonal sum makes $\mathscr{J}_{\mathbb{C}}$ into a symmetric monoidal category and $\mathscr{J}_{\mathbb{R}}$ an $\mathcal{T}^{C_{2}}$-enriched symmetric monoidal category. Using this one can define the smash product $X \wedge Y$ giving both $\mathcal{S}_{\mathbb{R}}$ and $\mathcal{S}_{\mathbb{C}}$ the structure of symmetric monoidal categories. The smash product in $\mathcal{S}_{\mathbb{R}}$ is specified by the formula

$$
S^{-V_{\mathbb{C}}} \wedge S^{-W_{\mathbb{C}}}=S^{-(V \oplus W)_{\mathbb{C}}}
$$

and the fact that it commutes with colimits in each variable. A similar characterization holds for $\mathcal{S}_{\mathbb{C}}$. 
There are indexed monoidal products in this context. Let $J$ be a finite set with a $C_{2}$-action. The actions of $C_{2}$ on $J$ and on $\mathcal{S}_{\mathbb{C}}$ combine to give an action on the product category $\mathcal{S}_{\mathbb{C}}^{J}$. The category of $\mathcal{S}_{\mathbb{R}}^{J}$ of real $J$-diagrams is the category of fixed points for this action. The category of real $J$-diagrams for $J=\{\mathrm{pt}\}$ is equivalent to $\mathcal{S}_{\mathbb{R}}$. When $J=C_{2}$, the category of real $J$-diagrams is equivalent to $\mathcal{S}_{\mathbb{C}}$. For general $J=n_{1}+n_{2} C_{2}$, one has an equivalence

$$
\mathcal{S}_{\mathbb{R}}^{J} \approx \mathcal{S}_{\mathbb{R}}^{n_{1}} \times \mathcal{S}_{\mathbb{C}}^{n_{2}}
$$

There are indexed wedges and indexed smash products from $\mathcal{S}_{\mathbb{R}}^{J}$ to $\mathcal{S}_{\mathbb{R}}$.

B.12.3. Homotopy theory of real and complex spectra. We now turn to the homotopy theory of real and complex spectra. We describe the case of $\mathcal{S}_{\mathbb{R}}$ and leave the analogous case of $\mathcal{S}_{\mathbb{C}}$ to the reader.

Suppose that $X$ is a real spectrum. For $H \subset C_{2}$ and $k \in \mathbb{Z}$ set

$$
\pi_{k}^{H}(X)=\underset{V}{\lim } \pi_{k+V_{\mathbb{C}}}^{H} X_{V_{\mathbb{C}}}
$$

The colimit is taken over the poset of finite dimensional orthogonal vector spaces over $\mathbb{R}$, ordered (in agreement with Definition 2.3) by dimension. A stable weak equivalence in $\mathcal{S}_{\mathbb{R}}$ is a map $X \rightarrow Y$ inducing an isomorphism $\pi_{k}^{H} X \rightarrow \pi_{k}^{H} Y$ for all $H \subset C_{2}$ and $k \in \mathbb{Z}$. For fixed $k$, the groups $\pi_{k}^{H}$ form a Mackey functor which we denote $\underline{\pi}_{k}$.

Equipped with the stable weak equivalences, the category $\mathcal{S}_{\mathbb{R}}$ becomes a homotopical category. We refine it to a model category by defining a map to be a fibration if for each non-zero $V$, the map $X_{V_{\mathbb{C}}} \rightarrow Y_{V_{\mathbb{C}}}$ is a fibration in $\mathcal{T}^{C_{2}}$. The cofibrations are the maps having the left lifting property against the acyclic fibrations. This is the positive stable model structure on $\mathcal{S}_{\mathbb{R}}$.

The positive stable model structure is cofibrantly generated. The generating cofibrations can be taken to be the maps of the form

$$
S^{-V_{\mathbb{C}}} \wedge\left(S_{+}^{n-1} \rightarrow D_{+}^{n}\right)
$$

and

$$
\left(C_{2}\right)_{+} \wedge S^{-V_{\mathbb{C}}} \wedge\left(S_{+}^{n-1} \rightarrow D_{+}^{n}\right)
$$

with $V>0$. The generating acyclic cofibrations are the analogous maps

$$
S^{-V_{\mathbb{C}}} \wedge\left(I_{+}^{n-1} \rightarrow I^{n}\right)
$$

and

$$
\left(C_{2}\right)_{+} \wedge S^{-V_{\mathbb{C}}} \wedge\left(I_{+}^{n-1} \rightarrow I^{n}\right)
$$

together with the corner maps formed by smashing

$$
S^{-V_{\mathbb{C}} \oplus W_{C}} \wedge S^{W_{\mathbb{C}}} \rightarrow \tilde{S}^{-V_{C}}
$$

with the maps $S_{+}^{n-1} \rightarrow D_{+}^{n}$ and $\left(C_{2}\right)_{+} \wedge\left(S_{+}^{n-1} \rightarrow D_{+}^{n}\right)$. We assume $V>0$, while $W$ need not be. The map (B.225) is extracted from the factorization

$$
S^{-V_{\mathbb{C}} \oplus W_{\mathbb{C}}} \wedge S^{W_{\mathbb{C}}} \rightarrow \tilde{S}^{-V_{\mathbb{C}}} \rightarrow S^{-V_{\mathbb{C}}}
$$

formed by applying the small object construction with the generating cofibrations. As in the case of the complete positive stable model structure on $\mathcal{S}^{G}$, the map 
$\tilde{S}^{-V_{\mathbb{C}}} \rightarrow S^{-V_{\mathbb{C}}}$ is a homotopy equivalence. The verification of the model category axioms is straightforward. See $§$ B.4.1 or [54].

B.12.4. Real spectra and $C_{2}$-spectra. Let

$$
i: \mathscr{J}_{\mathbb{R}} \rightarrow \mathscr{J}_{C_{2}}
$$

be the functor sending $V$ to

$$
V_{\rho_{2}}=V \otimes \rho_{2}
$$

Then the restriction functor

$$
i^{*}: \mathcal{S}^{C_{2}} \rightarrow \mathcal{S}_{\mathbb{R}}
$$

has both a left and right adjoint which we denote $i_{\text {! }}$ and $i_{*}$ respectively. The left adjoint sends $S^{-V_{\mathbb{C}}}$ to $S^{-V_{\rho_{2}}}$, and is described in general by applying the functor termwise to the tautological presentation.

Since the functor $i$ is symmetric monoidal, the left adjoint $i$ ! is strongly symmetric monoidal.

Proposition B.226. The functors

$$
i_{!}: \mathcal{S}_{\mathbb{R}} \leftrightarrows \mathcal{S}^{C_{2}}: i^{*}
$$

form a Quillen equivalence.

Remark B.227. A similar discussion leads to a Quillen equivalence

$$
\mathcal{S}_{\mathbb{C}} \leftrightarrows \mathcal{S}
$$

Proof: Since $i_{\text {! }}$ is a left adjoint and

$$
i_{!}\left(S^{-V_{\mathbb{C}}} \wedge A\right)=S^{-V_{\rho_{2}}} \wedge A
$$

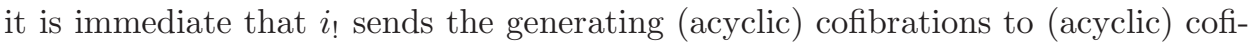
brations, and hence is a left Quillen functor. Using the fact that the sequence $\left\{\mathbb{R}^{n} \otimes \rho_{2}\right\}$ is exhausting, one can easily check that a map $X \rightarrow Y$ in $\mathcal{S}^{C_{2}}$ is a weak equivalence if and only if $i^{*} X \rightarrow i^{*} Y$ is. This means that to show that $i_{!}$and $i^{*}$ form a Quillen equivalence it suffices to show that the unit map

$$
X \rightarrow i^{*} i_{!} X
$$

is a weak equivalence for every cofibrant $X \in \mathcal{S}_{\mathbb{R}}$. Since $i^{*}$ is also a left adjoint, it preserves colimits, and therefore so does $i^{*} i$. Since both functors also commute with smashing with a $C_{2}$-space, we are reduced to checking that for each $0 \neq V \in \mathscr{J}_{\mathbb{R}}$, the map

$$
S^{-V_{\mathbb{C}}} \rightarrow i^{*} S^{-V_{\rho_{2}}}
$$

is a weak equivalence.

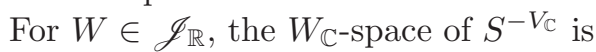

$$
\mathscr{J}_{\mathbb{R}}(V, W)=\operatorname{Thom}\left(U\left(V_{\mathbb{C}}, W_{\mathbb{C}}\right) ; W_{C}-V_{\mathbb{C}}\right)
$$

and the $W$-space of $i^{*} S^{-V_{\rho_{2}}}$ is

$$
\mathscr{J}_{C_{2}}\left(V_{\rho_{2}}, W_{\rho_{2}}\right)=\operatorname{Thom}\left(O\left(V_{\rho_{2}}, W_{\rho_{2}}\right) ; W_{\rho_{2}}-V_{\rho_{2}}\right) .
$$

The unit of the adjunction is derived from the inclusion $U\left(V_{\mathbb{C}}, W_{\mathbb{C}}\right) \rightarrow O\left(V_{\rho_{2}}, W_{\rho_{2}}\right)$. We must therefore show that for each $k$, the map

$$
\lim _{W \in \mathscr{J}_{\mathbb{R}}} \underline{\pi}_{k+W_{\mathbb{C}}} \mathscr{J}_{\mathbb{R}}\left(V_{\mathbb{C}}, W_{\mathbb{C}}\right) \rightarrow \underset{W \in \lim _{\underset{\mathbb{R}}{\longrightarrow}}}{\pi_{k+W_{\mathbb{C}}}} \mathscr{J}_{C_{2}}\left(V_{\rho_{2}}, W_{\rho_{2}}\right)
$$


is an isomorphism.

We may suppose that $\operatorname{dim} W>\operatorname{dim} V$. For a fixed $W$ choose an orthogonal embedding $V \subset W$, write $W=V \oplus U$, and consider the diagram

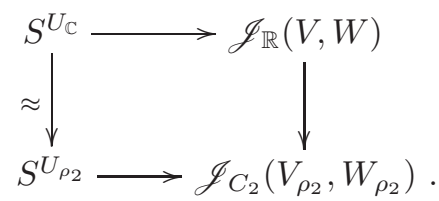

The left vertical map is an equivariant isomorphism. A straightforward argument using the connectivity of Stiefel manifolds shows that for $\operatorname{dim} W \gg 0$ the horizontal maps are isomorphisms in both $\pi_{k+W_{\mathbb{C}}}^{u}$ and $\pi_{k+W_{\mathbb{C}}}^{C_{2}}$. It follows that the right vertical map is as well, and hence so is (B.230).

For later reference, we record one fact that emerged in the proof of Proposition B.226.

Lemma B.231. The functor $i^{*}$ reflects weak equivalences: a map $X \rightarrow Y \in \mathcal{S}^{C_{2}}$ is a weak equivalence if and only if $i^{*} X \rightarrow i^{*} Y$ is.

B.12.5. Multiplicative aspects of real spectra. The multiplicative homotopy theory of real spectra is similar to that of $\mathcal{S}^{G}$. Though there does not seem to be a simple way to directly deduce the results from the case of $\mathcal{S}^{C_{2}}$, the proofs are very similar.

Proposition B.232. If $J$ is a set with a $C_{2}$-action and $X \rightarrow Y$ is a cofibration of cofibrant real J-diagrams, then both the indexed corner $\operatorname{map} \partial_{X} Y^{\wedge J} \rightarrow Y^{\wedge J}$ and the absolute map $X^{\wedge J} \rightarrow Y^{\wedge J}$ are cofibrations between cofibrant objects. They are weak equivalences if $X \rightarrow Y$ is.

Proof: This is an analogue of Propositions B.96 and B.103, and is proved in the same way, using the arrow category and the filtration of $\S$ A.3.4.

For the symmetric powers, we fix a $C_{2}$-set $J$ and a $C_{2}$-stable subgroup $\Sigma \subset \Sigma_{I}$. The following is an analog of Proposition B.117 and, making use of Proposition B.232, is proved in the same manner.

Proposition B.233. If $X \in \mathcal{S}_{\mathbb{R}}$ is cofibrant and $Z$ is any real spectrum equipped with an action of $\Sigma \rtimes C_{2}$ extending the $G$-action, then the map

$$
\left(E_{C_{2}} \Sigma\right)_{+} \hat{\Sigma}\left(X^{\wedge J} \wedge Z\right) \rightarrow\left(X^{\wedge J} \wedge Z\right) / \Sigma .
$$

is a weak equivalence.

Proposition B.234. If $A \rightarrow B$ is a cofibration of cofibrant real spectra and $J$ is a finite set with a $C_{2}$-action, then in the diagram

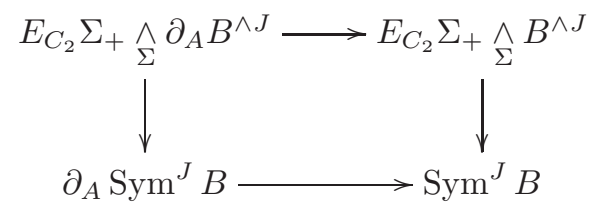

the upper row is a cofibration between cofibrant objects, the vertical maps are weak equivalences and remain so after smashing with any object, and the bottom row is an $h$-cofibration of flat spectra. The horizontal maps are weak equivalences if $A \rightarrow B$ $i s$. 
Proof: This is an analogue of Proposition B.109 and is proved in the same way, making use of Proposition B.233.

Proposition B.235. The forgetful functor

$$
\operatorname{comm} \mathcal{S}_{\mathbb{R}} \rightarrow \mathcal{S}_{\mathbb{R}}
$$

creates a model category structure on commutative algebras in $\mathcal{S}_{\mathbb{R}}$, in which a map of commutative algebras is a fibration or weak equivalence if and only if the underlying map of real spectra is.

Proof: This is proved in the same manner as Proposition B.130, making use of Proposition B.234.

B.12.6. Generalized flatness. Our next task is to show that the left derived functor of $i_{\text {! can }}$ be computed on a subcategory of real spectra containing those which underlie real commutative rings.

Definition B.236. A real spectrum $X \in \mathcal{S}_{\mathbb{R}}$ is $i_{\text {! }}$-flat if it satisfies the following property: for every cofibrant approximation $\tilde{X} \rightarrow X$ and every weak equivalence $\tilde{Z} \rightarrow Z \in \mathcal{S}^{C_{2}}$ the map

$$
i_{!} \tilde{X} \wedge \tilde{Z} \rightarrow i_{!} X \wedge Z
$$

is a weak equivalence.

Remark B.238. Since $i_{\text {! }}$ is a left Quillen functor and cofibrant objects of $\mathcal{S}^{C_{2}}$ are flat, cofibrant objects of $\mathcal{S}_{\mathbb{R}}$ are $i$ !-flat.

Remark B.239. If (B.237) is a weak equivalence for one cofibrant approximation it is a weak equivalence for any cofibrant approximation.

Our main result is

Proposition B.240. If $R \in \mathcal{S}_{\mathbb{R}}$ is a cofibrant commutative algebra then $R$ is $i_{!}$-flat.

The proof of Proposition B.240 follows the argument for the proof of Proposition B.147.

Lemma B.241. If $A \in \mathcal{S}_{\mathbb{R}}$ is cofibrant, and $n \geq 1$, then $\mathrm{Sym}^{n} A$ is $i_{!}$-flat.

Proof: By Proposition B.233, the map

$$
\left(E_{C_{2}} \Sigma_{n}\right)_{+} \underset{\Sigma_{n}}{\wedge} A^{\wedge n} \rightarrow \operatorname{Sym}^{n} A
$$

is a cofibrant approximation. Since $i_{\text {! }}$ is a continuous left adjoint, we may identify

$$
i_{!}\left(\left(E_{C_{2}} \Sigma_{n}\right)+\underset{\Sigma_{n}}{\wedge} A^{\wedge n}\right) \wedge \tilde{Z} \rightarrow i_{!}\left(\operatorname{Sym}^{n} A\right) \wedge Z
$$

with

$$
\left(E_{C_{2}} \Sigma_{n}\right)_{+} \wedge \Sigma_{\Sigma_{n}}\left(i_{!} A\right)^{\wedge n} \wedge \tilde{Z} \rightarrow \operatorname{Sym}^{n}\left(i_{!} A\right) \wedge Z .
$$

Since $i_{!}$is a left Quillen functor, $i_{!}(A)$ is cofibrant, and Proposition B.117 implies that (B.243), hence (B.242) is a weak equivalence.

We also require an analogue of Lemma B.164, though the statement and proof are much simpler in this case, since $i_{\text {! }}$ is a left adjoint. 
Lemma B.244. If $S \rightarrow T$ is an h-cofibration in $\mathcal{S}_{\mathbb{R}}$, and two of $S, T, T / S$ are $i_{!}$-flat, then so is the third.

Proof: We may choose a map $\tilde{S} \rightarrow \tilde{T}$ of cofibrant approximations which is a cofibration, hence an $h$-cofibration. Our assumption is that two of the vertical maps in

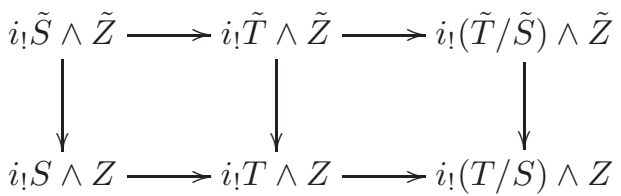

are weak equivalences. This implies that the third is, since the two left horizontal maps are $h$-cofibrations hence flat.

Lemma B.245. Consider a pushout square in $\mathcal{S}_{\mathbb{R}}$,

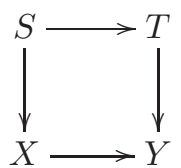

in which $S \rightarrow T$ is an $h$-cofibration. If $T, T / S$ and $X$ are $i_{!}$-flat, then so is $Y$.

Proof: Since $T$ and $T / S$ are $i_{!}$-flat, so is $S$ by Lemma B.244. We may choose cofibrant approximations of everything fitting into a pushout diagram

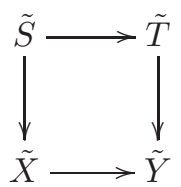

in which the top row is an $h$-cofibration. Now consider

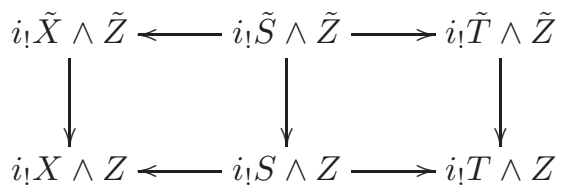

The left horizontal maps are $h$-cofibrations, hence flat, and the vertical maps are weak equivalences by assumption. It follows that the map of pushouts is a weak equivalence.

Proof of Proposition B.240: It suffices to show that if $A \rightarrow B$ is a generating cofibration in $\mathcal{S}_{\mathbb{R}}$ then

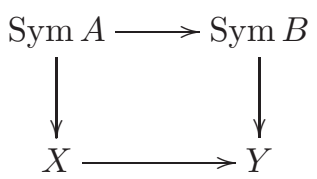


is a pushout square of commutative algebras in $\mathcal{S}_{\mathbb{R}}$ and $X$ is $i_{!}$-flat, then $Y$ is $i_{!}$-flat. We induct over the filtration described in $\S$ A.3.4. Since fil $l_{0} Y=X$, the induction starts. For the inductive step, consider the pushout square

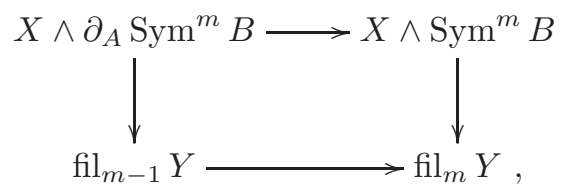

and assume that $\mathrm{fil}_{m-1} Y$ is $i_{!}$-flat. Both $\operatorname{Sym}^{m} B$ and

$$
\operatorname{Sym}^{m} B / \partial_{A} \operatorname{Sym}^{m} B=\operatorname{Sym}^{m}(B / A)
$$

are $i_{\text {! }}$-flat by Lemma B.241. Since smash products of $i_{\text {! }}$-flat spectra are $i_{\text {! }}$-flat, both $X \wedge \operatorname{Sym}^{m} B$ and $X \wedge \operatorname{Sym}^{m}(B / A)$ are $i_{\text {! }}$-flat. The top row of (B.247) is an $h$-cofibration, so Lemma B.245 implies that $\operatorname{fil}_{m} Y$ is $i_{!}$-flat. This completes the inductive step, and the proof.

Though we don't quite need the following result, having come this far we record it for future reference.

Proposition B.248. The functors $i_{!}$and $i^{*}$ restrict to a Quillen equivalence

$$
i_{!}: \operatorname{comm} \mathcal{S}_{\mathbb{R}} \leftrightarrows \operatorname{comm} \mathcal{S}^{C_{2}}: i^{*}
$$

Proof: It is immediate from the definition of the model structures on comm $\mathcal{S}_{\mathbb{R}}$ and $\operatorname{comm} \mathcal{S}^{C_{2}}$, and the fact that

$$
i_{!}: \mathcal{S}_{\mathbb{R}} \leftrightarrows \mathcal{S}^{C_{2}}: i^{*}
$$

is a Quillen pair, that

$$
i^{*}: \operatorname{comm} \mathcal{S}^{C_{2}} \rightarrow \operatorname{comm} \mathcal{S}_{\mathbb{R}}
$$

preserves the classes of fibrations and acyclic fibrations. It remains to show that if $A \in \operatorname{comm} \mathcal{S}_{\mathbb{R}}$ is cofibrant, then the composition

$$
A \rightarrow i^{*} i_{!} A \rightarrow i^{*}\left(i_{!} A_{f}\right)
$$

is a weak equivalence, where $i_{!} A \rightarrow i_{!} A_{f}$ is a fibrant replacement. Since $i^{*}$ reflects weak equivalences (Lemma B.231) this is equivalent to showing that

$$
A \rightarrow i^{*} i_{!} A
$$

is a weak equivalence. Let $A^{\prime} \rightarrow A$ be a cofibrant approximation in $\mathcal{S}_{\mathbb{R}}$, and consider the following diagram in $\mathcal{S}_{\mathbb{R}}$

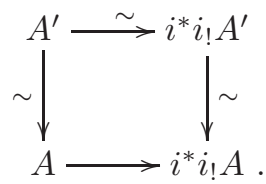

By Proposition B.240 the map $i_{!} A^{\prime} \rightarrow i_{!} A$ is a weak equivalence. The rightmost arrow in (B.249) is therefore a weak equivalence. The top arrow is a weak equivalence by Proposition B.226, and the left arrow is a weak equivalence by assumption. This implies that the bottom arrow is a weak equivalence. 
B.12.7. The real bordism spectrum. For $V \in \mathscr{J}_{\mathbb{R}}$ let

$$
M U\left(V_{\mathbb{C}}\right)=\operatorname{Thom}\left(B U\left(V_{\mathbb{C}}\right), V_{\mathbb{C}}\right)
$$

be the Thom complex of the bundle $E U\left(V_{\mathbb{C}}\right) \underset{U\left(V_{\mathbb{C}}\right)}{\times} V_{\mathbb{C}}$ over $B U\left(V_{\mathbb{C}}\right)$, equipped with the $C_{2}$-action of complex conjugation. We will take our model of $B U\left(V_{\mathbb{C}}\right)$ to be the one given by Segal's construction [77], so that

$$
V \mapsto \operatorname{Thom}\left(B U\left(V_{\mathbb{C}}\right), V_{\mathbb{C}}\right)
$$

is a lax symmetric monoidal functor $\mathscr{J}_{\mathbb{R}} \rightarrow \mathcal{I}_{C_{2}}$, and so defines a commutative ring $\mathcal{M U}_{\mathbb{R}} \in \mathbf{c o m m} \mathcal{S}_{\mathbb{R}}$. Let $\mathcal{M U}_{\mathbb{R}}^{\prime} \rightarrow \mathcal{M U}_{\mathbb{R}}$ be a cofibrant approximation to $\mathcal{M U}_{\mathbb{R}}$ in $\operatorname{comm} S_{\mathbb{R}}$.

Definition B.251. The real bordism spectrum is the spectrum $M U_{\mathbb{R}}=i_{!} \mathcal{M U}_{\mathbb{R}}^{\prime}$.

To get at the homotopy type of $M U_{\mathbb{R}}$, we examine the canonical homotopy presentation of $\mathcal{M U}_{\mathbb{R}}$ using the exhausting sequence $V_{n}=\mathbb{R}^{n}$. This gives a weak equivalence

$$
\operatorname{holim} S^{-\mathbb{C}^{n}} \wedge M U(n) \stackrel{\sim}{\longrightarrow} \mathcal{M U}_{\mathbb{R}}^{\prime}
$$

in which $M U(n)=M U\left(\mathbb{C}^{n}\right)$. Applying $i_{\text {! }}$ and using Proposition B.240 gives

$$
\operatorname{holim} S^{-n \rho_{2}} \wedge M U(n) \stackrel{\sim}{\longrightarrow} M U_{\mathbb{R}} \text {. }
$$

In this presentation the universal real orientation of $M U_{\mathbb{R}}$ (Example 5.5) is given by restricting to the term $n=1$

$$
S^{-\rho_{2}} \wedge M U(1) \rightarrow M U_{\mathbb{R}}
$$

The next result summarizes some further consequences of the presentation (B.252).

Proposition B.253. $\quad$ i) The non-equivariant spectrum underlying $M U_{\mathbb{R}}$ is the usual complex cobordism spectrum $M U$.

ii) The equivariant cohomology theory represented by $M U_{\mathbb{R}}$ coincides with the one studied in [43, 24, 6, 35].

iii) There is an equivalence

$$
\Phi^{C_{2}} M U_{\mathbb{R}} \approx M O
$$

iv) The Schubert cell decomposition of Grassmannians leads to a cofibrant approximation of $M U_{\mathbb{R}}$ by a $C_{2}-C W$ complex with one 0 -cell $\left(S^{0}\right)$ and the remaining cells of the form $e^{m \rho_{2}}$, with $m>0$.

\section{REFERENCES}

[1] J. Adámek, J. Rosický, and E. M. Vitale, What are sifted colimits?, Theory Appl. Categ. 23 (2010), No. 13, 251-260. MR 2720191 (2011g:18002)

[2] _ Algebraic theories, Cambridge Tracts in Mathematics, vol. 184, Cambridge University Press, Cambridge, 2011, A categorical introduction to general algebra, With a foreword by F. W. Lawvere. MR 2757312 (2012f:18001)

[3] J. F. Adams, On the non-existence of elements of Hopf invariant one, Ann. of Math. (2) 72 (1960), 20-104. MR MR0141119 (25 \#4530)

[4] J. F. Adams, Stable homotopy and generalised homology, University of Chicago Press, Chicago, 1974.

[5] J. F. Adams, Prerequisites (on equivariant stable homotopy) for Carlsson's lecture, Algebraic topology, Aarhus 1982 (Aarhus, 1982), Lecture Notes in Math., vol. 1051, Springer, Berlin, 1984, pp. 483-532. MR 764596 (86f:57037) 
[6] Shôrô Araki, Orientations in $\tau$-cohomology theories, Japan. J. Math. (N.S.) 5 (1979), no. 2, 403-430. MR MR614829 (83a:55010)

[7] M. F. Atiyah, K-theory and reality, Quart. J. Math. Oxford Ser. (2) 17 (1966), 367-386. MR 34 \#6756

[8] M. G. Barratt, J. D. S. Jones, and M. E. Mahowald, The Kervaire invariant problem, Proceedings of the Northwestern Homotopy Theory Conference (Providence, Rhode Island) (H. R. Miller and S. B. Priddy, eds.), Contemporary Mathematics, vol. 19, American Mathematical Society, 1983, pp. 9-22.

[9] — Relations amongst Toda brackets and the Kervaire invariant in dimension 62, Journal of the London Mathematical Society 30 (1985), 533-550.

[10] Andrew J. Blumberg and Michael A. Mandell, The homotopy theory of cyclotomic spectra, arXiv:1303.1694v2.

[11] Anna Marie Bohmann, A comparison of norm maps, Proc. Amer. Math. Soc. 142 (2014), no. 4, 1413-1423, With an appendix by Bohmann and Emily Riehl. MR 3162261

[12] Francis Borceux, Handbook of categorical algebra. 2, Encyclopedia of Mathematics and its Applications, vol. 51, Cambridge University Press, Cambridge, 1994, Categories and structures. MR 1313497 (96g:18001b)

[13] W. Browder, The Kervaire invariant of framed manifolds and its generalization, Annals of Mathematics 90 (1969), 157-186.

[14] Gunnar Carlsson, Equivariant stable homotopy and Segal's Burnside ring conjecture, Ann. of Math. (2) 120 (1984), no. 2, 189-224. MR 763905 (86f:57036)

[15] A. H. Clifford and G. B. Preston, The algebraic theory of semigroups. Vol. I, Mathematical Surveys, No. 7, American Mathematical Society, Providence, R.I., 1961. MR MR0132791 (24 \#A2627)

[16] Ethan S. Devinatz and Michael J. Hopkins, Homotopy fixed point spectra for closed subgroups of the Morava stabilizer groups, Topology 43 (2004), no. 1, 1-47. MR 2030586

[17] Ethan S. Devinatz, Michael J. Hopkins, and Jeffrey H. Smith, Nilpotence and stable homotopy theory. I, Ann. of Math. (2) 128 (1988), no. 2, 207-241. MR 89m:55009

[18] Andreas W. M. Dress, Contributions to the theory of induced representations, Algebraic $K$-theory, II: "Classical" algebraic $K$-theory and connections with arithmetic (Proc. Conf., Battelle Memorial Inst., Seattle, Wash., 1972), Springer, Berlin, 1973, pp. 183-240. Lecture Notes in Math., Vol. 342. MR MR0384917 (52 \#5787)

[19] E. Dror Farjoun, Cellular inequalities, The Čech centennial (Boston, MA, 1993), Contemp. Math., vol. 181, Amer. Math. Soc., Providence, RI, 1995, pp. 159-181. MR MR1320991 (96g:55011)

[20] Daniel Dugger, An Atiyah-Hirzebruch spectral sequence for KR-theory, K-Theory 35 (2005), no. 3-4, 213-256 (2006). MR MR2240234 (2007g:19004)

[21] William G. Dwyer, Philip S. Hirschhorn, Daniel M. Kan, and Jeffrey H. Smith, Homotopy limit functors on model categories and homotopical categories, Mathematical Surveys and Monographs, vol. 113, American Mathematical Society, Providence, RI, 2004. MR MR2102294 (2005k:18027)

[22] A. D. Elmendorf, I. Kriz, M. A. Mandell, and J. P. May, Rings, modules, and algebras in stable homotopy theory, American Mathematical Society, Providence, RI, 1997, With an appendix by M. Cole. MR 97h:55006

[23] Leonard Evens, A generalization of the transfer map in the cohomology of groups, Trans. Amer. Math. Soc. 108 (1963), 54-65. MR MR0153725 (27 \#3686)

[24] Michikazu Fujii, Cobordism theory with reality, Math. J. Okayama Univ. 18 (1975/76), no. 2, 171-188. MR MR0420597 (54 \#8611)

[25] Peter Gabriel and Friedrich Ulmer, Lokal präsentierbare Kategorien, Lecture Notes in Mathematics, Vol. 221, Springer-Verlag, Berlin, 1971. MR MR0327863 (48 \#6205)

[26] J. P. C. Greenlees and J. P. May, Equivariant stable homotopy theory, Handbook of algebraic topology, North-Holland, Amsterdam, 1995, pp. 277-323. MR MR1361893 (96j:55013)

[27] Localization and completion theorems for MU-module spectra, Ann. of Math. (2) 146 (1997), no. 3, 509-544. MR MR1491447 (99i:55012)

[28] A. Grothendieck, Revêtements étales et groupe fondamental (SGA 1), Documents Mathématiques (Paris) [Mathematical Documents (Paris)], 3, Société Mathématique de France, Paris, 2003, Séminaire de géométrie algébrique du Bois Marie 1960-61. [Algebraic Geometry Seminar of Bois Marie 1960-61], Directed by A. Grothendieck, With two papers by 
M. Raynaud, Updated and annotated reprint of the 1971 original [Lecture Notes in Math., 224, Springer, Berlin; MR0354651 (50 \#7129)]. MR MR2017446 (2004g:14017)

[29] Michael A Hill and Michael J Hopkins, Equivariant Multiplicative Closure, arXiv:1303.4479 [math.AT], March 2013.

[30] Philip S. Hirschhorn, Model categories and their localizations, Mathematical Surveys and Monographs, vol. 99, American Mathematical Society, Providence, RI, 2003. MR MR1944041 (2003j:18018)

[31] M. J. Hopkins and P. Goerss, Multiplicative stable homotopy theory, unpublished manuscript, 1996.

[32] Michael J. Hopkins and Jeffrey H. Smith, Nilpotence and stable homotopy theory. II, Ann. of Math. (2) 148 (1998), no. 1, 1-49. MR 99h:55009

[33] Mark Hovey, Smith ideals of structured ring spectra, arXiv:1401.2850.

[34] _ Model categories, Mathematical Surveys and Monographs, vol. 63, American Mathematical Society, Providence, RI, 1999. MR MR1650134 (99h:55031)

[35] Po Hu and Igor Kriz, Real-oriented homotopy theory and an analogue of the Adams-Novikov spectral sequence, Topology 40 (2001), no. 2, 317-399. MR MR1808224 (2002b:55032)

[36] Sören Illman, Smooth equivariant triangulations of $G$-manifolds for $G$ a finite group, Math. Ann. 233 (1978), no. 3, 199-220. MR 0500993 (58 \#18474)

[37] - The equivariant triangulation theorem for actions of compact Lie groups, Math. Ann. 262 (1983), no. 4, 487-501. MR 696520 (85c:57042)

[38] Peter T. Johnstone, Sketches of an elephant: a topos theory compendium. Vol. 1, Oxford Logic Guides, vol. 43, The Clarendon Press, Oxford University Press, New York, 2002. MR 1953060 (2003k:18005)

[39] John D. S. Jones, The Kervaire invariant of extended power manifolds, Topology 17 (1978), no. 3, 249-266. MR 508888 (80g:55026)

[40] Gregory Maxwell Kelly, Basic concepts of enriched category theory, London Mathematical Society Lecture Note Series, vol. 64, Cambridge University Press, Cambridge, 1982. MR 651714 (84e:18001)

[41] Michel A. Kervaire, A manifold which does not admit any differentiable structure, Comment. Math. Helv. 34 (1960), 257-270.

[42] Michel A. Kervaire and John W. Milnor, Groups of homotopy spheres. I, Ann. of Math. (2) 77 (1963), 504-537.

[43] Peter S. Landweber, Conjugations on complex manifolds and equivariant homotopy of $M U$, Bull. Amer. Math. Soc. 74 (1968), 271-274. MR MR0222890 (36 \#5940)

[44] Homological properties of comodules over $M \mathrm{U}_{*}(M \mathrm{U})$ and $B P_{*}(B P)$, Amer. J. Math. 98 (1976), no. 3, 591-610. MR 0423332 (54 \#11311)

[45] Miguel L. Laplaza, Coherence for distributivity, Coherence in categories, Springer, Berlin, 1972, pp. 29-65. Lecture Notes in Math., Vol. 281. MR 0335598 (49 \#379a)

[46] J. P. Levine, Lectures on groups of homotopy spheres, Algebraic and geometric topology (New Brunswick, N.J., 1983), Lecture Notes in Math., vol. 1126, Springer, Berlin, 1985, pp. 62-95. MR 802786 (87i:57031)

[47] G. Lewis, J. P. May, and J. McClure, Ordinary RO $(G)$-graded cohomology, Bull. Amer. Math. Soc. (N.S.) 4 (1981), no. 2, 208-212. MR 598689 (82e:55008)

[48] L. G. Lewis, J. P. May, and M. Steinberger, Equivariant stable homotopy theory, Lecture Notes in Mathematics, vol. 1213, Springer-Verlag, New York, 1986.

[49] J. Lubin and J. Tate, Formal complex multiplication in local fields, Annals of Mathematics 81 (1965), 380-387.

[50] Saunders Mac Lane and Ieke Moerdijk, Sheaves in geometry and logic, Universitext, SpringerVerlag, New York, 1994, A first introduction to topos theory, Corrected reprint of the 1992 edition. MR MR1300636 (96c:03119)

[51] Saunders MacLane, Categories for the working mathematician, second ed., Graduate Texts in Mathematics, vol. 5, Springer-Verlag, New York, 1998. MR 1712872 (2001j:18001)

[52] Mark Mahowald and Martin Tangora, Some differentials in the Adams spectral sequence, Topology 6 (1967), 349-369. MR MR0214072 (35 \#4924)

[53] M. A. Mandell and J. P. May, Equivariant orthogonal spectra and S-modules, Mem. Amer. Math. Soc. 159 (2002), no. 755, x+108. MR MR1922205 (2003i:55012)

[54] M. A. Mandell, J. P. May, S. Schwede, and B. Shipley, Model categories of diagram spectra, Proc. London Math. Soc. (3) 82 (2001), no. 2, 441-512. MR MR1806878 (2001k:55025) 
[55] Michael A. Mandell, Equivariant symmetric spectra, Homotopy theory: relations with algebraic geometry, group cohomology, and algebraic $K$-theory, Contemp. Math., vol. 346, Amer. Math. Soc., Providence, RI, 2004, pp. 399-452. MR 2066508 (2005d:55019)

[56] J. P. May, The cohomology of restricted Lie algebras and of Hopf algebras, Ph.D. thesis, Princeton University, 1964.

[57] J. P. May, Equivariant homotopy and cohomology theory, CBMS Regional Conference Series in Mathematics, vol. 91, Published for the Conference Board of the Mathematical Sciences, Washington, DC, 1996, With contributions by M. Cole, G. Comezaña, S. Costenoble, A. D. Elmendorf, J. P. C. Greenlees, L. G. Lewis, Jr., R. J. Piacenza, G. Triantafillou, and S. Waner. MR MR1413302 (97k:55016)

[58] J. Peter May, $E_{\infty}$ ring spaces and $E_{\infty}$ ring spectra, Springer-Verlag, Berlin, 1977, With contributions by Frank Quinn, Nigel Ray, and Jørgen Tornehave, Lecture Notes in Mathematics, Vol. 577. MR 58 \#13008

[59] M. C. McCord, Classifying spaces and infinite symmetric products, Trans. Amer. Math. Soc. 146 (1969), 273-298. MR 0251719 (40 \#4946)

[60] R. James Milgram, Group representations and the Adams spectral sequence, Pacific J. Math. 41 (1972), 157-182. MR 0304463 (46 \#3598)

[61] H. R. Miller, On relations between Adams spectral sequences, with an application to the stable homotopy of a Moore space, Journal of Pure and Applied Algebra 20 (1981), 287-312.

[62] H. R. Miller and D. C. Ravenel, Morava stabilizer algebras and the localization of Novikov's $E_{2}$-term, Duke Mathematical Journal 44 (1977), 433-447.

[63] H. R. Miller, D. C. Ravenel, and W. S. Wilson, Periodic phenomena in the Adams-Novikov spectral sequence, Annals of Mathematics 106 (1977), 469-516.

[64] Haynes Miller, Kervaire invariant one [after M. A. Hill, M. J. Hopkins, and D. C. Ravenel], Astérisque (2012), no. 348, Exp. No. 1029, vii, 65-98, Séminaire Bourbaki: Vol. 2010/2011. Exposés 1027-1042. MR 3050712

[65] J. W. Milnor, On the cobordism ring $\Omega^{*}$ and a complex analogue, Part I, American Journal of Mathematics 82 (1960), 505-521.

[66] J. Morava, Noetherian localizations of categories of cobordism comodules, Annals of Mathematics 121 (1985), 1-39.

[67] D. G. Quillen, On the formal group laws of unoriented and complex cobordism theory, Bulletin of the American Mathematical Society 75 (1969), 1293-1298.

[68] _ Homotopical algebra, Lecture Notes in Mathematics, vol. 43, Springer-Verlag, New York, 1972

[69] Daniel Quillen, Elementary proofs of some results of cobordism theory using Steenrod operations, Advances in Math. 7 (1971), 29-56 (1971). MR MR0290382 (44 \#7566)

[70] D. C. Ravenel, The nonexistence of odd primary Arf invariant elements in stable homotopy theory, Math. Proc. Cambridge Phil. Soc. 83 (1978), 429-443.

[71] _ Complex cobordism and stable homotopy groups of spheres, second ed., AMS Chelsea Publishing, vol. 347, American Mathematical Society, 2004.

[72] Charles Rezk, Fibrations and homotopy colimits of simplicial sheaves, arXiv:math/9811038.

[73] _ Notes on the Hopkins-Miller theorem, Homotopy theory via algebraic geometry and group representations (Evanston, IL, 1997), Contemp. Math., vol. 220, Amer. Math. Soc., Providence, RI, 1998, pp. 313-366. MR MR1642902 (2000i:55023)

[74] Stefan Schwede, An untitled book project about symmetric spectra, Available at http://www .math.uni-bonn.de/people/schwede/.

[75] Stefan Schwede and Brooke Shipley, Equivalences of monoidal model categories, Algebr. Geom. Topol. 3 (2003), 287-334 (electronic). MR MR1997322 (2004i:55026)

[76] Stefan Schwede and Brooke E. Shipley, Algebras and modules in monoidal model categories, Proc. London Math. Soc. (3) 80 (2000), no. 2, 491-511. MR MR1734325 (2001c:18006)

[77] G. Segal, Classifying spaces and spectral sequences, Inst. Hautes Études Sci. Publ. Math. 34 (1968), 105-112.

[78] Katsumi Shimomura, Novikov's Ext ${ }^{2}$ at the prime 2, Hiroshima Math. J. 11 (1981), no. 3, 499-513. MR MR635034 (83c:55027)

[79] Tammo tom Dieck, Transformation groups and representation theory, Lecture Notes in Mathematics, vol. 766, Springer, Berlin, 1979. MR 551743 (82c:57025)

[80] _ Transformation groups, de Gruyter Studies in Mathematics, vol. 8, Walter de Gruyter \& Co., Berlin, 1987. MR 889050 (89c:57048) 
[81] Vladimir Voevodsky, Open problems in the motivic stable homotopy theory. I, Motives, polylogarithms and Hodge theory, Part I (Irvine, CA, 1998), Int. Press Lect. Ser., vol. 3, Int. Press, Somerville, MA, 2002, pp. 3-34. MR MR1977582 (2005e:14030)

[82] _ A possible new approach to the motivic spectral sequence for algebraic $K$-theory, Recent progress in homotopy theory (Baltimore, MD, 2000), Contemp. Math., vol. 293, Amer. Math. Soc., Providence, RI, 2002, pp. 371-379. MR MR1890744 (2003g:55011)

[83] _ On the zero slice of the sphere spectrum, Tr. Mat. Inst. Steklova 246 (2004), no. Algebr. Geom. Metody, Svyazi i Prilozh., 106-115. MR MR2101286 (2005k:14042)

[84] Klaus Wirthmüller, Equivariant homology and duality, Manuscripta Math. 11 (1974), 373390. MR MR0343260 (49 \#8004)

Department of Mathematics, University of Virginia, Charlottesville, VA 22904

E-mail address: michael.a.hill@math.uva.edu

Department of Mathematics, Harvard University, Cambridge, MA 02138

E-mail address: mjh@math.harvard.edu

Department of Mathematics, Rochester University, Rochester, NY

E-mail address: doug@math.rochester.edu 